ORNL/TM-12434

\title{
Atmospheric Sampling Glow Discharge lonization and Triple Quadrupole Tandem Mass Spectrometry for Explosives Vapor Detection
}

Final Report for work performed for the Federal Aviation Administration Technical Center, Atlantic City, NJ, under Interagency Agreement DTFA03-90-A-00004.

\author{
Scott A. McLuckey \\ Douglas E. Goeringer \\ Keiji G. Asano \\ Kevin J. Hart \\ Gary L. Glish \\ Barry C. Grant \\ David M. Chambers
}

Date Published - August 1993

\author{
Prepared by the \\ Analytical Chemistry Division \\ OAK RIDGE NATIONAL LABORATORY \\ Oak Ridge, Tennessee 37831-6285 \\ managed by \\ MARTIN MARIETTA ENERGY SYSTEMS, INC. \\ for the \\ U.S. DEPARTMENT OF ENERGY \\ under Contract No. DE-AC05-84OR21400




\section{TABLE OF CONTENTS}

Page

I. Introduction $\ldots \ldots \ldots \ldots \ldots \ldots \ldots \ldots \ldots \ldots$

II. Executive Summary $\ldots \ldots \ldots \ldots \ldots \ldots \ldots \ldots$

III. Atmospheric Sampling Glow Discharge Ionization $\ldots \ldots \ldots \ldots \ldots$

A. ASGDI for High Explosives Detection $\ldots \ldots \ldots \ldots \ldots \ldots$

B. The Role of Gas Dyrnamics in Anion Formation in ASGDI . . . 13

IV. Tandem Mass Spectrometry $\ldots \ldots \ldots \ldots \ldots \ldots \ldots \ldots \ldots$

A. Beam-type Technologies ................. 38

B. Ion Trapping Technologies ................43

V. The FAA Glow Discharge/Triple Quadrupole Tandem Mass Spectrometer . . . . . . . . . . . . . . . . . . . . 57

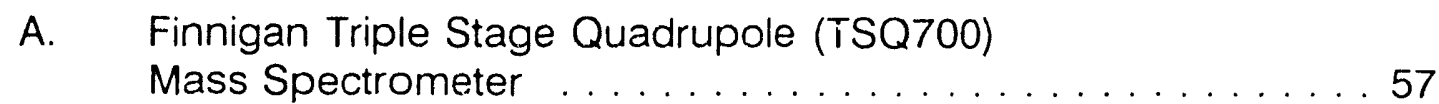

B. Scan Capabilities ......................663

C. ASGDI-TSQ700 Interface . . . . . . . . . . . . . . . . .65

VI. Performarce Characteristics of the FAA ASGDI/TSQ700 . . . . . . . . . . . . . . . . . . . . 71

A. Mass Spectrometry $\ldots \ldots \ldots \ldots \ldots \ldots \ldots \ldots \ldots$

B. Mass Spectrometry/Mass Spectrometry $\ldots \ldots \ldots \ldots \ldots 76$

C. Sensitivities in Various Operating Modes ...........81

D. Automated Explosives Detertion . . . . . . . . . . . . . . . 85

VII. Comparison of ASGDI on Various Tandem Mass

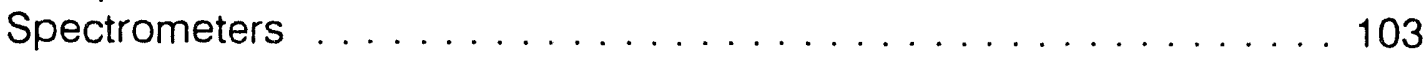


Table of Contents - Continued

Page

VIII. Integration of the FAA Glow Discharge/Triple Quadrupole Tandem Mass Spectrometer with the Ion Track Instruments Rotary Trap Vapor Preconcentrator . . . . . . . . . . . . . . . . . . . 113

A. Ion Track Instruments Rotary Trap Vapor Preconcentrator . . 113

B. Performance Characteristics of the Rotary Trap/ASGDI/TSQ700 System . . . . . . . . . . . . . . . . 115

IX. Summary and Prognosis . . . . . . . . . . . . . . . . . 123

X. References ... . . . . . . . . . . . . . . . . . . . . . . . . . 125

XI. Appendix I ........................ 131

XII. Appendix II .............................. 135 


\section{LIST OF FIGURES}

Page

Figure 1. A side-view schematic of the ASGDI source attached to a quadrupole mass filter. . . . . . . . . . . . . . . . . 9

Figure 2. Cutaway side view of the ASGDI source interface region. . . . . 15

Figure 3. Diagram depicting the different flow region of the free-jet expansion that forms in the ASGDI source chamber. . . . . . 18

Figure 4. Full mass scans of ambient air monitoring of DNT taken at several skimming distances: (a) 8 , (b) 10, (c) 12 , and (d) $14 \mathrm{~mm}$

Figure 5. Dual graph depicting minimum source pressure required to detect DNT (M) anion and the distance skimming takes place behind the Mach disk as skimming distance is varied.

Figure 6. Relative abundance of the five most intense anions obtained from ambient air monitoring of DNT as the source pressure is increased.

Figure 7. Effect of higher cathode voltages on relative anion signals of ambient air monitoring of DNT.

Figure 8. Full-mass scan of ambient air monitoring of DNT at a source pressure of 0.67 Torr and cathode voltage of $-436 \mathrm{~V}$

Figure 9. DNT signal at two lateral skimming positions with respect to the expansion axis, on-axis and $1 \mathrm{~mm}$ off-axis, as the source pressure is varied.

Figure 10. Identical analysis as performed in Figure 9 except the skimming distance has been extended to $12 \mathrm{~mm}$.

Figure 11. Schematic comparison of GC/MS with MS/MS.

Figure 12. Schematic representation of the ASGDI MS/MS experiment for explosives detection.

Figure 13. Some common MS/MS instrumental configurations. 
List of Figures - Continued

Page

Figure 14. Photograph of the quadrupole ion trap assembly; two endcap electrodes and a ring electrode. . . . . . . . . . . . . 45

Figure 15. Schematic representation of the electric fields experienced by an ion for a large positive voltage and for a large negative voltage.

Figure 16. Side-view schematic of the ion trap with a sinusoidal signal applied to the end-caps to effect resonance ejection.

Figure 17. MS/MS spectra of $\mathrm{m} / \mathrm{z} 240,\left(\mathrm{M}-\mathrm{CH}_{2} \mathrm{ONO}_{2}\right)$, from PETN (top) spectrum of isolated parent ion (bottom) spectrum after resonance excitation.

Figure 18. Block diagram of the TSQ 700 instrument (unmodified). 58

Figure 19. Side-view schematic of the ASGDI coupled to the

Figure 20. Front view of the ASGDI source assembly attached to the TSQ 700 .

Figure 21. Full view of the TSQ 700 with the ASGDI source assembly installed.

Figure 22. Mass spectra obtained on the ASGDI/TSQ700 for TNT, PETN, and RDX. . . . . . . . . . . . . . . . . 72

Figure 23. ASGDI/TSQ700 background mass spectrum from laboratory air.

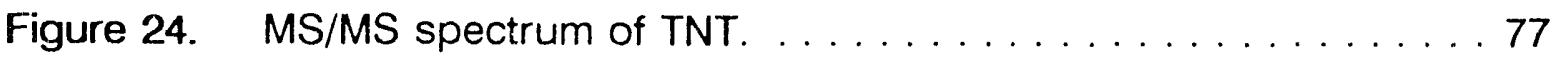

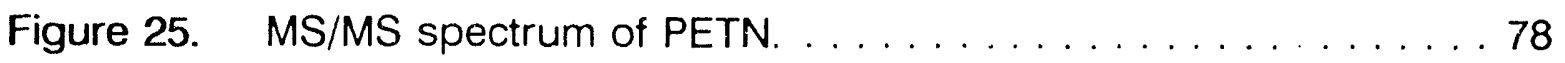

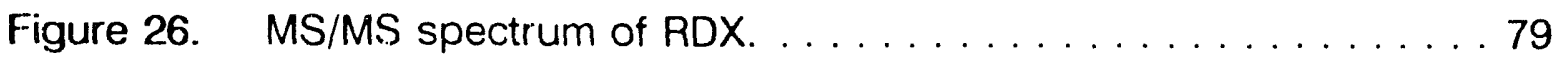

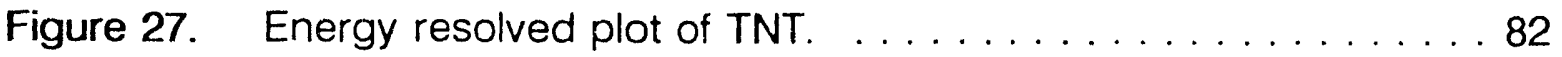


List of Figures - Continued

Page

Figure 28. Targeted product ion mass spectra for TNT. . . . . . . . 83

Figure 29. Photograph of the TSQ 700 with ASGDI source assembly anú associated work station. . . . . . . . . . 86

Figure 30. Typical screen display showing various instrument control windows for operation of the TSQ 700. . . . . . . 88

Figure 31. Example of an ICL procedure. . . . . . . . . . . . 91

Figure 32. Example of an ICL procedure using other procedures as commands. . . . . . . . . . . . . . . . . 92

Figure 33. Guide window showing icon "buttons" allowing simple push-button operation ................ 94

Figure 34. Screen display of the main menu of the ORNL Explosives Detector application . . . . . . . . . . . . . . 95

Figure 35. Flow chart of algorithm used for automated explosives detection on the ASGDI/TSQ700.

Figure 36. Split screen display when monitoring mode selected, status clear. . . . . . . . . . . . . . . . . . . . . . . . 98

Figure 37. Split screen display when possible explosive found. . . . . . . 99

Figure 38. Split screen display when explosive confirmed by MS/MS. . . 101

Figure 3r Side-view schematic of the ASGDI source coupled with the quadrupole ion trap. . . . . . . . . . . . . . . . . . . 104

Figure 40. Schematic diagram of the ASGDI source and quadrupole/time-of-flight $\ldots \ldots \ldots \ldots \ldots$

Figure 41. MS/MS of TNT vs. Instrument $\ldots \ldots \ldots \ldots 7$

Figure 42. $\mathrm{MS} / \mathrm{MS}$ of PETN vs. Instrument $\ldots \ldots \ldots \ldots \ldots$

Figure 43. MS/MS of RDX vs. Instrument . . . . . . . . . . . 109 
List of Figures - Continued

Page

Figure 44. A schematic drawing (top view) of the backing plate for the sampling head of the rotary trap $\ldots \ldots \ldots \ldots \ldots 114$

Figure 45. Plot of peak area for $\mathrm{m} / \mathrm{z} 176$ from RDX versus injected volume. . . . . . . . . . . . . . . . . . . . 116

Figure 46. Headspace vapor analysis of TNT in a vial . . . . . . 118

Figure 47. Headspace vapor analysis of TNT vial in a heated box . . . . 121

Figure 48. Triplicate 1 minute sampling experiments with TNT $\ldots \ldots . .122$ 


\begin{abstract}
The detection and identification of trace vapors of hidden high explosives is an excellent example of a targeted analysis problem. It is desirable to push to ever lower levels the quantity or concentration of explosives material that provides an analytical signal, while at the same time discriminating against all other uninteresting material. The detection system must therefore combine high sensitivity with high specificity. Work in the Analytical Chemistry Division of Oak Ridge National Laboratory, including effort supported under this interagency agreement, has focussed on the development of instrumentation to address this problem. This report describes the philosophy behind the use of atmospheric sampling glow discharge ionization, which is a sensitive, rugged, and convenient means for forming anions from explosives molecules, with tandem mass spectrometry, which provides unparalleled specificity in the identification of explosives-related ions. Atmospheric sampling glow discharge ionization and tandem mass spectrometry are described in turn with particular emphasis on research results obtained under this agreement. Forms of tandem mass spectrometry are compared and contrasted to provide a summary of the characteristics to be expected from an explosives detector employing mass spectrometry/mass spectrometry. The instrument developed for the FAA, an atmospheric sampling glow discharge/triple quadrupole mass spectrometer, is described in detail with particular emphasis on the ion source/spectrometer interface and on the capabilities of the spectrometer. Performance characteristics of the system are also described as they pertain to explosives of interest including a description of an automated procedure for the detection and identification of specific explosives. A comparison of various tandem mass spectrometers mated with atmospheric sampling glow discharge is then described to illustrate the points made in the background sections and preliminary studies with a vapor preconcentration system provided by the FAA will be described. Finally a short synopsis of the current capabilities of the FAA glow discharge/tandem mass spectrometer is given along with a prognosis of the most promising research and development directions both for atmospheric sampling glow discharge and tandem mass spectrometry as they pertain to explosives detection.
\end{abstract}




\section{INTRODUCTION}

The detection and identification of trace vapors of hidden high explosives is a textbook example of a targeted analysis problem analogous to finding the proverbial needle in a haystack. Inherent to the approach of explosives detection via the trace vapor analysis is the indirect connection between the quantity of airborne material that might be available for analysis and the size and location of

a bomb. It is therefore desirable to push to ever lower levels the quantity or concentration of explosives material that provides an analytical signal to maximize the "true positive" and "true negative" rates of the detection system. At the same time, the detection system must be capable of discriminating against all other uninteresting material to minimize the "false positive" and "false negative" rates. The system must therefore combine high sensitivity with high specificity. Other highly important criteria include analysis time, the size and weight of the system, and the associated equipment, manpower, and training costs. Work in the Analytical Chemistry Division of Oak Ridge National Laboratory, including effort supported under this interagency agreement, has focussed on the combination of atmospheric sampling glow discharge ionization with tandem mass spectrometry. Atmospheric sampling glow discharge provides a simple, rugged, and convenient means for forming anions from explosives molecules while tandem mass spectrometry provides unparalleled specificity in the identification of explosivesrelated ions.

This report describes the philosophy behind the use of atmospheric sampling glow discharge with tandem mass spectrometry for explosives detection along with the relative merits of various possible incarnations of the combination. Atmospheric sampling glow discharge and tandem mass spectrometry are described in turn with particular emphasis on research results obtained under this agreement. Forms of tandem mass spectrometry are compared and contrasted to provide a summary of the characteristics to be expected from an explosives detector employing mass spectrometry/mass spectrometry. The instrument 
developed for the FAA, an atmospheric sampling glow discharge/triple quadrupole mass spectrometer, is described in detail with particular emphasis on the ion source/spectrometer interface and on the capabilities of the spectrometer. Performance characteristics of the system are also described as they pertain to explosives of interest including a description of an automated procedure for the detection and identification of specific explosives. A comparison of various tandem mass spectrometers mated with atmospheric sampling glow discharge is then described to illustrate the points made in the background sections and preliminary studies with a vapor preconcentration system provided by the FAA will be described. Finally a short synopsis of the current capabilities of the FAA glow discharge/tandem mass spectrometer is given along with a prognosis of the most promising research and development directions both for atmospheric sampling glow discharge and tandem mass spectrometry as they pertain to explosives detection. 


\section{II.EXECUTIVE SUMMARY}

Atmospheric sampling glow discharge ionization (ASGDI) coupled with tandem mass spectrometry is a highly specific and sensitive approach to the detection of trace quantities of high explosives in ambient air. Under this agreement, research in ASGDI and development of an ASGDI/triple quadrupole mass spectrometer was performed.

ASGDI is particularly useful for explosives detection in that high number densities of slow electrons are present in the discharge to provide for extensive electron capture. Organic high explosives tend to have unusually large crosssections for electron capture whereas most atoms and molecules do not. Furthermore, the pumping rate in the ionization region can be controlled to discriminate for or against products formed from ion/molecule reactions. In the explosives detection application, it is usually desirable to discriminate against ion/molecule reactions. Under this situation, ionization is under kinetic control rather than thermodynamic control. That is, ionic products observed in the mass spectrum are those species which are formed fastest. Electron capture tends to be one hundred to one thousand times faster than the fastest ion/molecule reactions. Operating the glow discharge source at pumping speeds of several liters/second minimizes the possibility for ion/molecule reactions while allowing for electron/molecule reactions. An important result is that the ASGDI source operated in this manner is relatively free of matrix effects upon ionization. That is, the presence of traces of uninteresting species, such as solvents, perfumes, etc., tend to have very little effect on the ions derived from explosives.

Research results derived in part from support under this agreement have also indicated that gas dynamics has an important effect on negative ion formation in ASGDI. The free jet expansion associated with air drawn into the ASGDI source through the inlet aperture creates regions with high probabilities for anion formation and regions with low probabilities for anion formation. The results indicate that, in particular, the refiected shock region is amenable for electron 
capture whereas the zone of silence produces no negative ions. In fact, the discharge is not expected to be able to penetrate into this region of high pressure. These results indicate, therefore, that there are preferred locations for sampling ions from the discharge for mass spectrometric analysis. Specifically, several millimeters downstream from the Mach disk and within one or two millimeters of the central axis defined by the inlet and exit apertures of the ion source is the optimal region for sampling ions formed primarily by electron capture.

Tandem mass spectrometry is a particularly specific means for analyzing ions formed from targeted compounds. There is a wide variety of instrumental approaches to tandem mass spectrometry or mass spectrometry/mass spectrometry (MS/MS) that can be divided conveniently as either beam-type or trapping technologies. Both beam-type and ion trapping technologies have been coupled with ASGDI for explosives detection. Until very recently, the more mature beam-type technologies were most suitable for real-world targeted compound detection. Recent advances in ion trapping technologies irvolving the quadrupole ion trap, however, indicate significant advantages in sensitivity, size, and cost. Furthermore, serious limitations for real-world targeted compound analysis imposed by finite ion storage capacity have been significantly mitigated. The triple quadrupole approach to tandem mass spectrometry, as employed in the system developed for the FAA, and the quadrupole ion trap approach are compared and contrasted at more length within the report. Benchmark studies were also performed with the triple quadrupole system, an ion trap system, and a quadrupole/time-of-flight system built at ORNL. The triple quadrupole system proved to be more sensitive and more specific than the quadrupole/time-of-flight system under every operational mode. However, the ion trap proved to be one to two orders of magnitude more sensitive than either beam-type technology with equal or superior specificity. However, at some sacrifice in specificity and scan range, the triple quadrupole system could yield sensitivities comparable to those observed with the ion trap. 
The basis for the FAA ASGDI/triple quadrupole tandem mass spectrometer is the Triple Stage Quadrupole 700 (TSQ700) manufactured by Finnigan Corporation of San Jose, CA. The instrument actually consists of two quadrupole mass filters separated by an octapole collision cell. The instrument is referred to as a "triple quadrupole" because the first commercially available tandem quadrupole instruments employed a non-mass-analyzing quadrupole as the collision cell. The major modification made to this system for air sampling explosives delection was the removal of the conventional chemical ionization/electron ionization ion source and replacement with an atmospheric sampling glow discharge source along with the associated power supply and pumpi ig. The system now constitutes a very powerful analytical instrument for detection and identification of trace quantities of targeted compounds in ambient air The instrument is capable of a variety of scanning modes each of which enjoys advantages for particular screening situations. These include, for example, the usual product ion scan, the parent ion scan, the neutral loss scan, and the targeted product scan. The latter is a high sensitivity screening mode developed at ORNL for explosives detection and allows the ASGDI/TSQ700 to compete with the ion trap, albeit at some cost in specificity and scan range. All of the scan modas of potential interest for explosives detection including, of course, acquiring the conventional mass spectrum have been evaluated with explosives of interest, suct, is 2,4,6 trinitrotoluene. Performance levels achieved are those to be expected with a good interface between ion source and analyzer. Depending upon scan mode, detection limits can range from sub-picogram to several picograms for high explosives.

As part of this contract, the TSQ700 was programmed for automatic explosives detection. The program was designed for an extremely simple user interaction comprised of three icon "buttons". The three buttons are labelled TUNE, START, and RESET and each is activated via a mouse. The TUNE button Initiates a series of procedures that tune the spectrometer and calibrate the mass scale and requires the admission of a calibration compound. This procedure also 
performs the procedures called by the RESET button. This button characterizes the intensities of the background ions at the mass/charge values of interest so that signal/background measurements can be made in near real time. The START button initiates a set of procedures that continually monitors the signal levels for whatever parent ion mass/charge values have been selected. When a particular parent ion exceeds a pre-defined intensity, mass spectrometry/mass spectrometry is performed on the ion. During this procedure a warning is indicated on the screen that a possible explosive has been identified. If the expected product ions are observed at a signal/background ratio in excess of five, the screen indicates that an explosive is detected. The MS/MS spectrum is displayed on part of the screen and a flashing red light is activated. The data are stored so that the particular explosive can be identified and the event can be documented and a hard copy of the screen showing the MS/MS spectrum is sent to a copier.

Preliminary system integration studies were performed to combine a vapor preconcentrator with the ASGDI/TSQ700 instrument. These studies involved a rotary trap preconcentrator developed by lon Track Instruments of Wilmington, MA. This preconcentrator was developed for electron capture detection. The goal of this work was to determine what benefit might accrue with such a preconcentrator mated with the mass spectrometer system. A major difference between the electron capture and mass spectrometer detectors lies in the inlet flow rates of the respective devices. The mass spectrometer inlet flow rate exceeds that of the electron capture detector by one to two orders of magnitude. The potential concentration effect is limited by the ratio of the inlet flow rates of the preconcentrator and detector. In the case of the ASGDI/TSQ700, this ratio was about twenty. Experimentally, the preconcentrator appeared to provide neither a positive nor deleterious effect on analyte signal levels other than a somewhat increased time required for response. This result seems to indicate that more extensive analyte losses are occurring with the present set-up than was previously observed with the rotary preconcentrator coupled with an electron capture detector. 


\section{ATMOSPHERIC SAMPLING GLOW DISCHARGE IONIZATION}

In any application of mass spectrometry the choice of ionization method is of critical importance. Since matter comes in all forms, i.e. solid, liquid, gas, and ranges from atoms to small polyatomic molecules to large biomolecules, a wide variety of ionization methods has been developed for mass spectrometry. For the detection of explosives vapors, therefore, gas-phase ionization methods are employed. A variety of such methods has been applied to the analysis and detection of explosives vapors including electron ionization [1-4], chemical ionization [1-3], field ionization [1,2], atmospheric pressure ionization [1-2,4-6], low pressure electron capture [7,8], and glow discharge ionization [9]. Each method has its own set of characteristics relative to the detection of explosives vapors. It is beyond the scope of this report to discuss the relative merits of each of the possible approaches to ionizing trace explosives vapors in air. Rather, the merits of atmospheric sampling glow discharge ionization (ASGDI) are discussed here with occasional reference to how these characteristics compare with those of other methods. In general, ASGDI is a simple and rugged means for ionizing organic species in air in either the positive or negative ion modes. The response is essentially instantaneous with essentially no memory effects arising from analyte species that enter tine ionization region. Work at ORNL has shown it to be useful for a variety of applications in trace vapors analysis. It is particularly useful for applications in which negative ion analysis is appropriate, as it is with high explosives.

\section{A. ASGDI for High Explosives Detection}

The explosives vapor detection problem requires the formation of ions characteristic of explosives when they are present in a complex mixture. The mixture could be air sampled directly into the detector or a mixture resulting from a preconcentration step and subsequently introduced into the detector. The relative concentrations of mixture components, particularly at the parts per million 
level and below, can vary widely with both location and time. Since it is desirable to detect explosives vapors present at parts per trillion levels and below, an extremely selective ionization method is mandated. The analysis of negative ions in the explosives detection application is employed because, unlike most compounds in nature, high explosives such as the nitroaromatics, the nitrate esters, and the heterocyclic nitramines, readily form anions. Indeed, several other approaches to explosives vapor detection take advantage of this characteristic. These include ion mobility spectrometry (IMS) [10], atmospheric pressure ionization mass spectrometry (APIMS) [4-6, 11-15], and gas chromatography/electron capture detection (GC/ECD) [16]. There are, however, differences between the conditions used in the ASGDI source and those used in the other devices that have important ramifications for the ions that are observed and the susceptibility for interferences. These points are discussed following a brief description of the ASGDI source and its operating conditions as it has been most commonly employed.

A side-view schematic of the ASGDI source attached to a quadrupole mass filter is shown in Figure 1. The ionization region in this case is contained within a 6 " Conflat flange. A $13 / 4^{\prime \prime}$ diameter hole is cut through the center of the flange. Four $1 / 2$ " pumpout ports are drilled through the side of the flange. Two aperture plates $A 1$ and $A 2$ are attached to either side of the Conflat flange and are electrically isolated from the flange by Viton O-rings. The O-rings also serve as vacuum seals. A1 typically contains a $200 \mu \mathrm{m}$ aperture in its center and is attached to the atmosphere side of the flange. A2 contains a 400-800 $\mu \mathrm{m}$ aperture in its center and is attached to the vacuum side of the flange. The flange is typically pumped at a rate of $\approx 8 \mathrm{~L} / \mathrm{s}$ which gives a pressure in the ionization region of $\approx 0.7-0.9$ Torr. Under these conditions, ambient air is drawn into the flange at a rate of $\approx 3-5 \mathrm{~mL} / \mathrm{s}$. A potential of $\approx-400 \mathrm{~V}$ is applied to $\mathrm{A} 1$ while $\mathrm{A} 2$ is typically grounded. Under these conditions a glow discharge spontaneously begins between $A 1$ (cathode) and A2 (anode). Note that the ASGDI developed for the FAA tandem mass spectrometer differs somewhat with respect to vacuum 


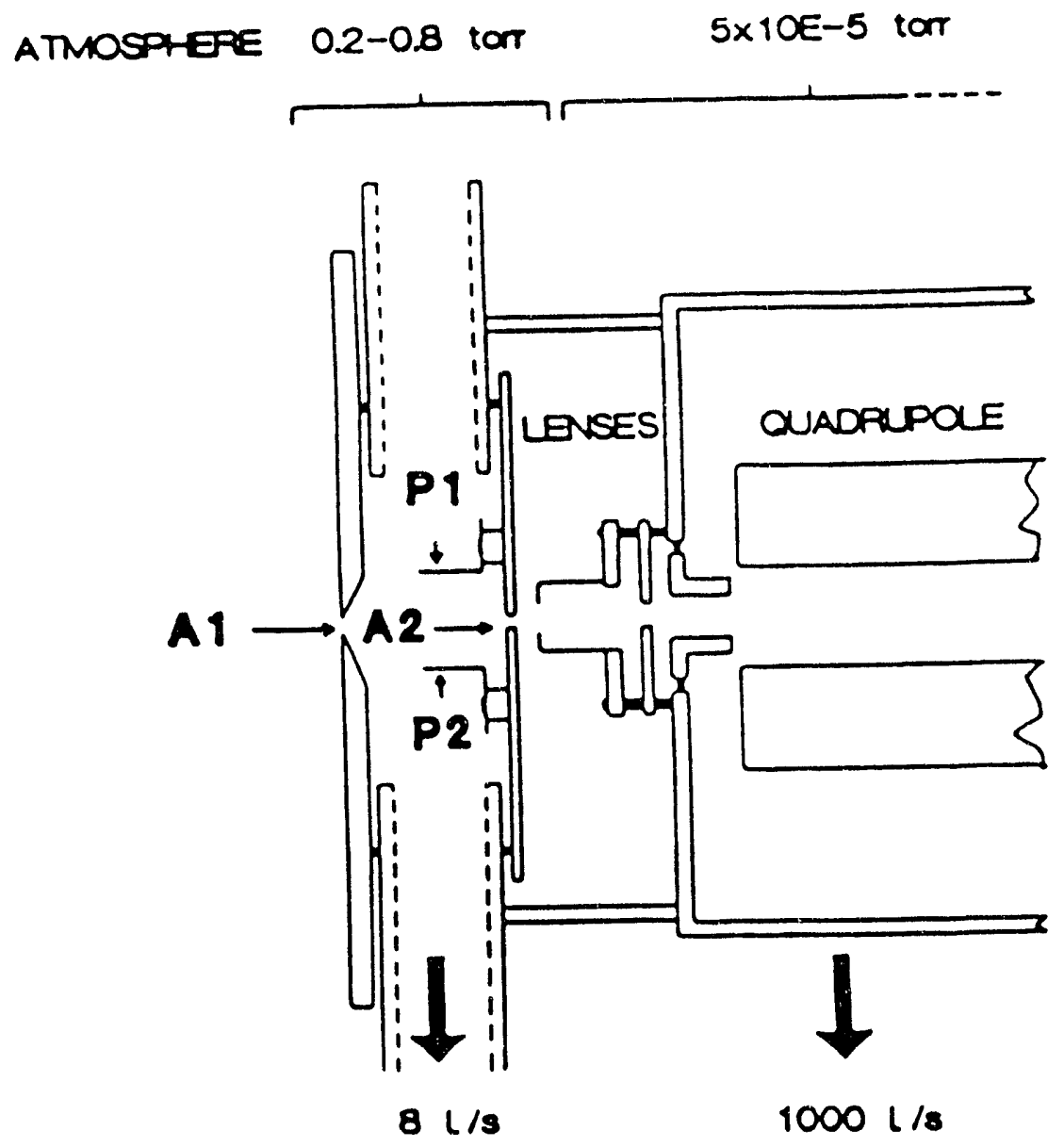

Figure 1. A side-view schematic of the ASGDI source attached to a quadrupole mass filter. 
hardware but the working conditions are essentially identical to those described above.

As indicated above, the conditions used in the ASGDI source are significantly different from those used in ionization at atmospheric pressure. These differences and their effects can be appreciated by considering simple pseudo-first order kinetics. Consider the ion/molecule reaction

$$
X+M \cdots>X+M
$$

where analyte species $M$ is present at a constant concentration throughout the period the reaction can proceed. The number of $X$ reagent ions observed after some reaction time $\mathrm{t}$ is given by

$$
[X]=[X]_{0} e^{-k i M) i}
$$

and the number of $M$ ions that are formed is given by

$$
[M]=[X]_{0}\left(1-e^{-k[M] t}\right)
$$

where $[X]_{0}$ is the number of $X$ ions present at $t=0, k$ is the rate constant for the ion/molecule reaction, and $[M]$ is the number density of $M$. Under API conditions, flow rates are typically on the order of $1 \mathrm{~mL} / \mathrm{s}$ giving reaction (residence) times on the order of $1 \mathrm{~s}$. Rate constants for fast ion/molecule reactions are on the order of $10^{-9} \mathrm{~cm}^{3} /$ molec-s. Rate constants for electron capture reactions, i.e.,

$$
e+M \cdots M
$$

can be as high as $10^{7} \mathrm{~cm}^{3} /$ molec-s (100 times greater). Electrons are therefore quickly captured in the presence of an electronegative reagent gas forming anions. (The number density of electronegative gases (primarily $\mathrm{O}_{2}$ ) in air at atmospheric pressure and room temperature is $\approx 4 \times 10^{18} \mathrm{~cm}^{-3}$.) The number densities of the reactants and the long reaction time result in a distribution of ion/molecule reaction product ions that tend to reflect thermodynamic equilibrium. Provided the rate constants are not too low, both the forward and reverse reactions for an ion/molecule reaction can occur a sufficient number of times to give an equilibrium distribution of reactants and products. Furthermore, number densities are high enough for third-body collisions to occur resulting in the observation of stable adduct ions as indicated below: 


$$
\mathrm{X}+\mathrm{M}+\mathrm{N}-\mathrm{M}+\mathrm{MX}+\mathrm{N} .
$$

The likelihood for observation of cluster ions in APIMS is further enhanced by the expansion of the plasma from atmosphere into the high vacuum. For this reason, ion declustering methods are typically employed.

In the ASGDI source, number densities are roughly three orders of magnitude lower than in API devices. Furthermore, the residence time of the molecules in the ionization region is typically less than $1 \mathrm{~ms}$ (as determined by the ventilation rate). Given such short residence times, ion/molecule reactions can only proceed to an appreciable extent if, assuming a rate constant of $10^{-9}$ $\mathrm{cm}^{3} /$ molec-s, the neutral number density is greater than about 20 parts per billion. Product ions begin to rival the intensities of the background "reagent ions" in the source $(\approx 10 \%$ of the total ion current) only when the concentration of the analyte is at least 20 parts per million. Therefore, for species present at levels less than $1 \mathrm{ppm}$ the vast majority of negative ions come from electron capture and not ion/molecule reactions.

These differences in operating conditions have several significant effects on the data acquired with ASGDI and API ion sources. The first is that the ions characteristic of the explosive may be different. For API sources it is common to observe $(M-H)$ and $(M+X)$ ions whereas with the ASGDI source it is common to observe $\mathrm{M}$ and/or fragment ions. The second effect manifests itself in the susceptibility for interferences. There are typically two types of interferences with most analytical techniques. One occurs when some species other than the analyte gives a signal that is indistinguishable from that of the analyte (tandem mass spectrometry is used to minimize this interference as discussed below). The second type occurs when some species alters the signal from the analyte either by suppressing its intensity or by moving it to another part of the spectrum where it is not recognized. An example of the latter would occur when some background species enters the API ionization region in sufficient quantity to change the ion/molecule reaction chemistry leading to a change in the identity of the analyterelated ions. This may involve a change, for example, from $(M+X)$ to $(M+Y)$. 
Since ion/molecule reaction chemistry plays essentially no role for low concentration analytes in the ASGDI source, the ions associated with the explosives do not change position on the mass scale despite the presence of relatively large doses of compounds that alter ion/molecule reaction chemistry. The ASGDI source is vulnerable to this type of interference only insofar as it affects the number of electrons and distribution of their kinetic energies. An extreme example occurs when a heavy dose ( $>10$ parts per thousand) of a halocarbon is used to extinguish the discharge. Our experience with halocarbons at levels insufficient to extinguish the discharge but sufficient to completely alter the distribution of background ions in the ion source is a diminution in signal due to explosives of a factor of 2-3 but no change in the identity of the ions. The ASGDI source, therefore, is relatively immune to interferences of the second type.

Clustering is much less likely in ASGDI than in API for some of the reasons just mentioned. Clustering of analyte ions with analyte molecules is unlikely due both to the short residence time and the low concentration levels of analyte. Clustering with background species, such as water, is also rarely observed due to the fact that the initial expansion from atmosphere into vacuum occurs prior to ionization rather than after ionization as in API. Therefore, the ion-dipole and ioninduced dipole attraction that promotes cluster formation around an ion under expansion conditions is not operative in the ASGDI. The second expansion from the ASGDI source into the mass spectrometer region is too dilute for significant clustering.

The characteristics of ASGDI just discussed and others that make this approach to ionization attractive for real-time explosives detection are summarized briefly as follows:

Ruggedness - The ASGDI has no filaments or discharge needles to replace and can be operated continuously for months without maintenance. Despite rather extensive ion burning, source cleaning is not important. Some of the ion sources at ORNL have been 
operated over the course of several years without cleaning and show no loss in performance.

- High throughput - The air sampling rate is $2-5 \mathrm{~mL} / \mathrm{s}$ which is relatively high for a vapor detector. This makes for a rapid response and a rapid clear-out of analyte. Memory effects are solely due to adhesion on surfaces leacing into the ion source.

- Kinetic vs. thermodynamic control - Ionization is under kinetic control which makes it less prone to matrix effects than ion sources primarily under thermodynamic control, e.g. API.

- Sensitive - For species with positive electron affinities, the ASGDI source coupled with several different mass spectrometers has been shown to give detection limits in the low part per trillion, femtogram range for high explosives.

- $\quad$ Selective - Inherent in negative ion analysis is the selectivity arising from the limited number of species that form stable anions. This selectivity is enhanced further by selecting for species that tend to be formed directly by electron capture rather than by ion/molecule reactions.

\section{B. The Role of Gas Dynamics in Anion Formation in ASGDI}

The ASGDI sources used at ORNL for explosives detection share the common characteristic of in-line apertures $A 1$ and $A 2$ that serve as the orifices that interface atmosphere with the discharge region (A1) and the discharge region with the vacuum system of the mass spectrometer (A2). We have noted in our studies that the appearance of the mass spectrum can be significantly affected by the size of $A 1$, the distance between $A 1$ and $A 2$, the pressure in the discharge region, and pumping speed. The mass spectra can vary both in the total number of ions and in the relative contributions to total anion current due to species formed from ion/molecule reactions and those formed from electron capture. Many of these observations can be rationalized based on changes in neutral number densities 
and on residence times. However, we have also noted that the expansion of gas from atmosphere into the glow discharge can affect mass spectra. For example, when humid room temperature air is drawn into an ASGDI source constructed like that shown in Figure 1, significant intensities of ions arising from $\mathrm{O}_{2}\left(\mathrm{H}_{2} \mathrm{O}\right)$ and $\mathrm{O}_{2}\left(\mathrm{H}_{2} \mathrm{O}\right)_{2}$ are observed in the mass spectrum. When the same air is heatcd to about $40^{\circ} \mathrm{C}$, the oxygen-hydrates disappear. Furthermore, when $\mathrm{A} 1$ is increased to a diameter of $3 \mathrm{~mm}$ and air is admitted to it via a leak valve to maintain the same pressure in the glow discharge region, no cluster ions are observed. Aside from the appearance of these ions in the mass spectrum, the presence of a free jet expansion extending into the discharge might also have a significant effect on electron energies and number densities in the plasma. Due to the higher number density in the expansion region, it is unlikely that the plasma can extend into the expansion itself. Therefore, it seemed highly probable that anions are most likely to be formed in specific regions of the ASGDI source and that there might be preferred regions in which to place the ion sampling aperture (A2). For these reasons, we constructed a specialized ASGDI source to study the role of gas dynamics on the formation of explosives related anions [17].

\section{Experimental Conditions}

The ASGDI source shown in Figure 2 is based on the version shown in Figure 1 with several new features added to increase the operational flexibility and to allow for the study of hydrodynamic processes. The sampling plate, the plate that contains $\mathrm{A} 1$, has a $200 \mu \mathrm{m}$ diameter orifice and serves as the cathode. The back aperture was replaced by a skimmer cone with a $0.8 \mathrm{~mm}$ diameter orifice. Each of these parts is electrically isolated from the source chamber by bolting them down with nylon screv:s on top of a Viton O-ring. Provisions are made so the spacing between the sampling plate and skimmer cone can be varied from 0 to $20 \mathrm{~mm}$. The sampling plate mount is threaded and can be screwed into the source chamber, which is $3.75 \mathrm{in}$. in diameter. Charged species are restricted to a region near the ion optical axis by an interface coil that is also electrically 

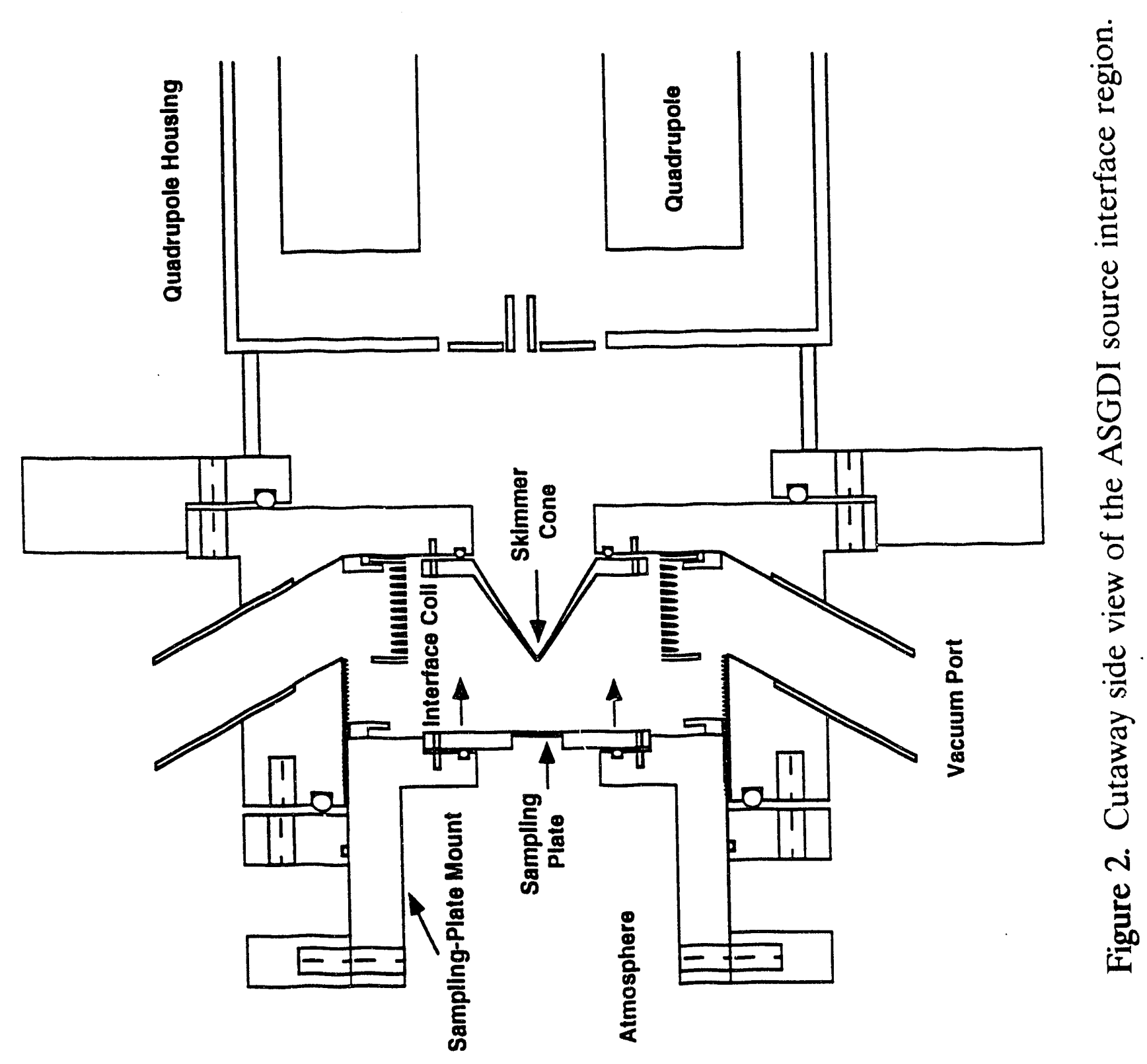
iscilated. Best negative ion signals are obtained when the coil is held at ground potential.

For these experiments, a $15 \mathrm{~L} / \mathrm{s}$ mechanical pump was connected to the ASGDI source through two of the four 1-in. diameter vacuum flanges. The source pressure could be adjusted by inrottling the flow at the entrance of the pump with a butterfly valve. Under these conditions, the lowest obtainable source pressure with no throttle was approximately 0.50 Torr as measured with a Baratron capacitance manometer that was connected into one of the other vacuum flanges. This pressure closely corresponds to a calculated value of 0.48 Torr when no conductance limit is assumed for the system.

The quadrupole mass filter (Extrel C-50 system) used for these studies was modified by removing all the ion optic lenses except for the ELFS aperture. The mass analyzer vacuum chamber was evacuated by a $1000 \mathrm{~L} / \mathrm{s}$ turbomolecular pump. Under normal operating conditions the backing pressure fell within the $10^{4}$ to $10^{5}$ Torr range. Throughout these studies 2,4-dinitrotoluene (DNT) served as the test compound and was sampled by placing approximately $1 \mathrm{mg}$ of the solid into a $20 \mathrm{~mL}$ vial and setting it near the sample inlet of the source. Analyte signal could be attenuated by moving the vial farther from the sample inlet or loosely screwing a lid on the vial.

\section{Negative Ion Formation in ASGDI}

Ionization mechanisms that are expected to occur in ASGDI arise from glowdischarge sustaining processes, which include ion bombardment of the cathode, elastic or inelastic collisions among charge species and neutrals, and photon- or collision-induced excitation [18]. Each of these processes varies according to source pressure, type of bath gas, and electrode potentials to produce several distinct regions that have been found to promote either positive or negative ion formation. According to the classical description of the glow discharge, positive ion formation occurs mainly in the negative-glow region near the cathode where both high- and low-energy electrons excite and ionize the discharge species. 
Closer to the anode in the positive column, electrons that have become slowed from inelastic collisions are able to form negative ions through electron capture. The general mechanisms that are accessible to the ASGDI technique for negative ion production are summarized below:

$$
\begin{array}{ll}
A+e \rightarrow A & \text { electron capture }(E C) \\
R+A \rightarrow R+A & \text { charge transfer } \\
R+A H \rightarrow R H+A & \text { proton abstraction } \\
R+A \rightarrow R A & \text { ion-molecule association }
\end{array}
$$

where $A$ is the sample molecule and $R$ is a reactant anion. The electron capture channels that are available include dissociative attachment and radiative or collisional stabilization. In ASGDI our aim is to optimize operating parameters to promote anion formation, yet minimize dissociative processes as well as ionmolecule association (clustering).

Glow discharge processes in the ASGDI geometries currently employed for anion formation are more complex due to the hydrodynamics of the free-jet expansion associated with the gas inlet via A1. The expansion is comprised of several zones as shown in Figure 3 where local density and flow velocity differ as well as conditions that might promote a specific reaction pathway $[19,20]$. As species proceed downstream from the sampling plate, flow velocity increases in the axial direction and both number density and collisional rate rapidly drop off. Eventually, the density of expanding gas becomes low enough to permit the penetration of thermalized background gas, thus, forming a high collisional zone that surrounds the expansion core. In this region, referred to as the shockwave structure, gas density abruptly rises and the expanding species are decelerated. For either the continuum flow region or expansion core to be included as part of the glow-discharge sustaining region, ion and electron penetration has to occur. Although electron migration into the expansion likely takes place, the penetration of ions is confined to the shockwave structure as are other background species.

The glow discharge can be sustained in the shockwave structure, which is comprised of two regions that have different gas dynamic properties. In the 


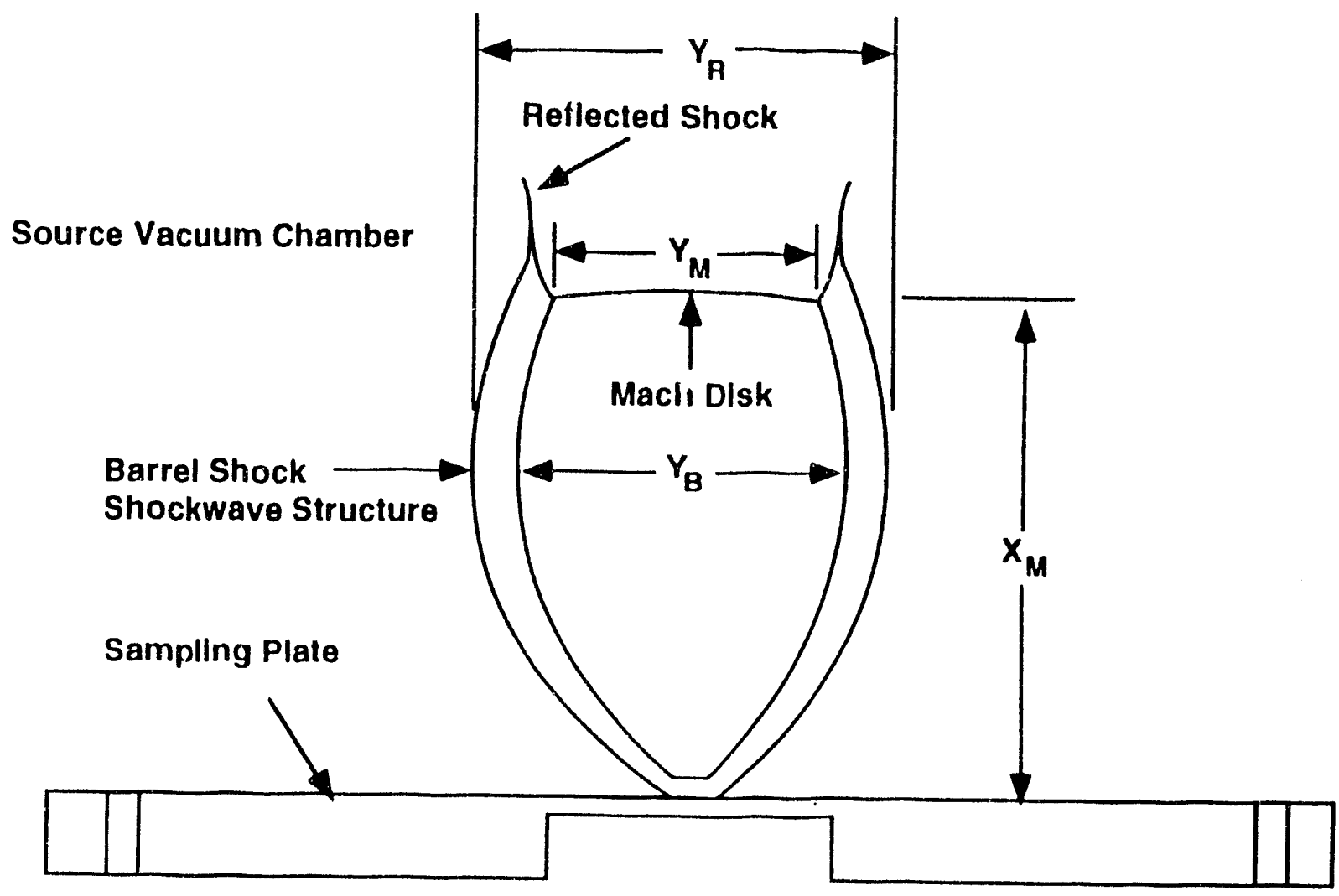

Continuum Flow

Atmosphere

Figure 3. Diagram depicting the different flow region of the free-jet expansion that forms in the ASGDI source chamber. 
region that forms perpendicular to the mean molecular flow (referred to as the Mach disk) exists a higher gas density and collisional frequency than the one that forms parallel (called the barrel shock) [21]. Those species that enter the barrel shock are able to maintain a larger part of their on-axis velocity component and travel beyond the Mach disk, forming the reflected shock. In the experiments described below, where skimming occurs many millimeters behind the Mach disk, the majority of those species sampled are believed to be derived from the barrel shock rather than the Mach disk.

The dimensions of the free-jet expansion can be varied by adjusting the source pressure. For example, increasing the source pressure reduces the expansion volume and compresses the shockwave structure. These dimensions can be approximated from experimentally determıned values [22]. Calculated values that are compiled in Table I correspond to a nitrogen expansion through a $0.2-\mathrm{mm}$ orifice from a 760 Torr reservoir to a 0.48 Torr chamber. At a source pressure of 0.48 Torr, which is the thecretical limit for the system, the free-jet boundary, $Y_{k}$ is expected to be $3.0 \mathrm{~mm}$ in diameter. Dimensions have also been determined at other pressures and will be cited as they pertain to this study.

\begin{tabular}{|c|c|c|c|}
\hline \hline \multicolumn{3}{|c|}{ Table I. Dimensions of Free-Jet Expansions } \\
\cline { 2 - 4 } $\begin{array}{c}\text { dimension } \\
\text { parameter }\end{array}$ & \multicolumn{3}{|c|}{ source pressure (Torr) } \\
\cline { 2 - 4 } & 0.48 & 0.75 & 0.90 \\
\hline$Y_{B}$ & 2.6 & 2.1 & 1.9 \\
\hline$Y_{M}$ & 2.1 & 1.7 & 1.6 \\
\hline$Y_{A}$ & 3.0 & 2.4 & 2.3 \\
\hline$X_{M}$ & 5.3 & 4.3 & 3.9 \\
\hline
\end{tabular}

The above description reveals two possibilities as to the effect of hydrodynamic flow on glow discharge, iocesses. The shockwave structure might serve as an ion-molecule reaction zone that can be manipulated to promote a 
particular ion-formation mechanism or the expansion only exists as a source of hydrodynamic perturbation that interferes with normal glow-discharge operation.

Different regions of the expansion can be skimmed axially either by mechanically adjusting the skimming position or by altering the source backing pressure thereby changing the expansion dimensions. Both these approaches were implemented to determine the effect of hydrodynamic flow on negative ion formation as well as to reveal mechanistic pathways and optimal operating parameters for negative ion formation.

\section{Contribution of the Expansion to Anion Formation}

Plotted in Figure 4 are spectra taken at selected skimming distances with the cathode voltage at $-370 \mathrm{~V}$ and the source pressure at 0.75 Torr. These particular conditions were chosen for illustration because the optimal combination of skimming distance and source pressure for DNT (M) anion signal was found to be $12 \mathrm{~mm}$ and 0.75 Torr, respectively. When the skimming distance was $8 \mathrm{~mm}$ or less essentially no ion signals were observed. At greater skimming distances the DNT $(M)$ anion signal maximized, followed by a rise in air-matrix related species (i.e., $\mathrm{O}, \mathrm{O}_{2}, \mathrm{NO}_{2}$, and $\mathrm{CO}_{3}$ ), and then declined. Among the conclusions to be drawn from these results is that anion formation is restricted some distance behi. $d$ the Mach disk, which is calculated to be only $4.3 \mathrm{~mm}$ downstream from the expansion orifice ( 0.75 Torr source pressure). As skimming is moved farther downstream, DNT anions are lost, perhaps, as a result of ion-molecule reactions that give rise to more thermodynamically favored products.

The next group of measurements was performed to determine the minimum distance behind the Mach disk where the formation of DNT anion begins. The Mach disk, which is characterized by an abrupt rise in gas density and collisional rate, was initially suspected of being a region of high electron-ion-molecule reactivity. Because varying the source pressure also changes the expansion dimensions, the skimmer-Mach disk separation was determined at several skimming distances as a function of source pressure. Results presented in 

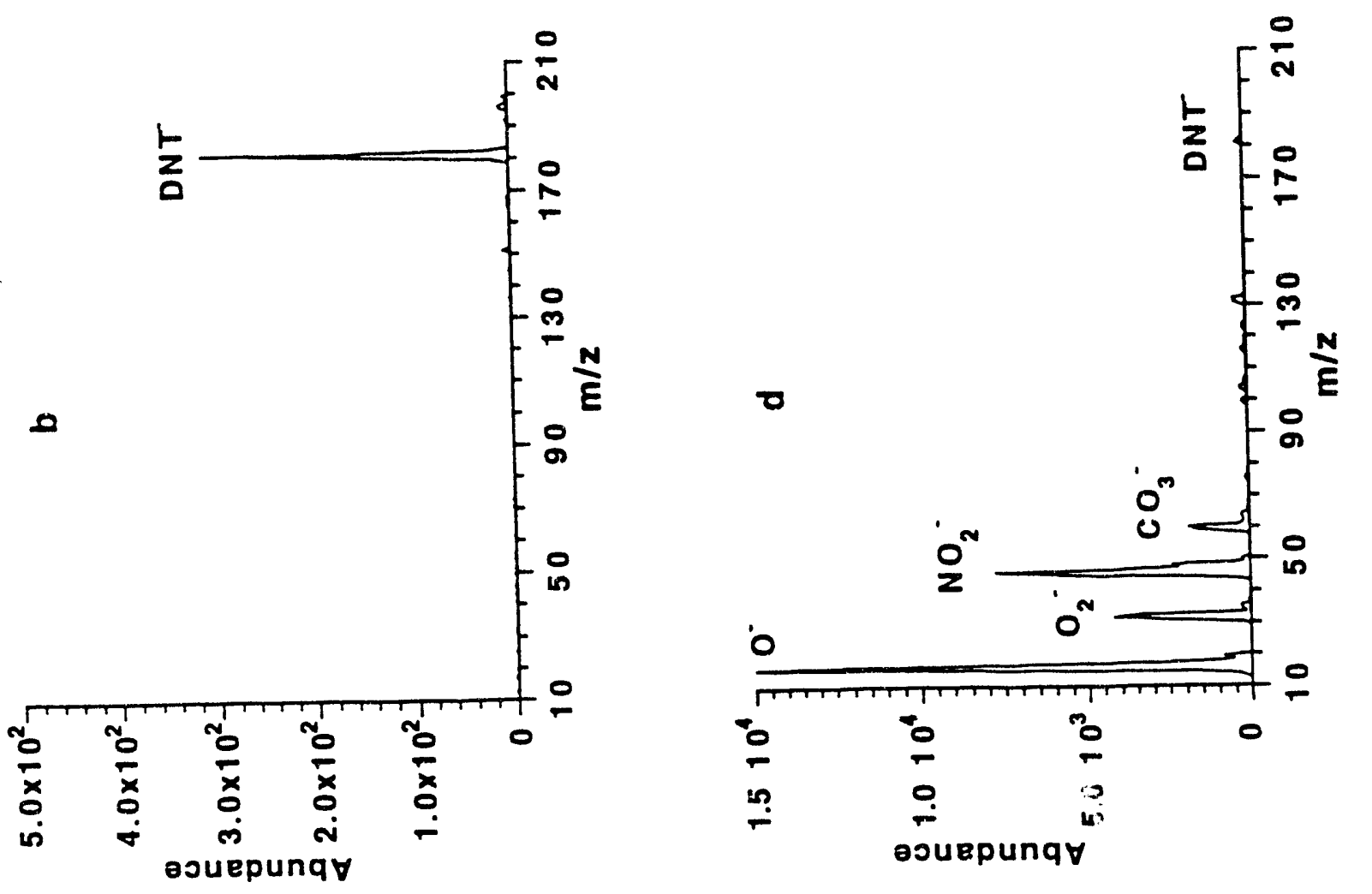

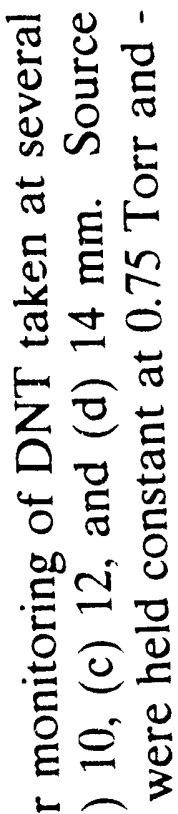
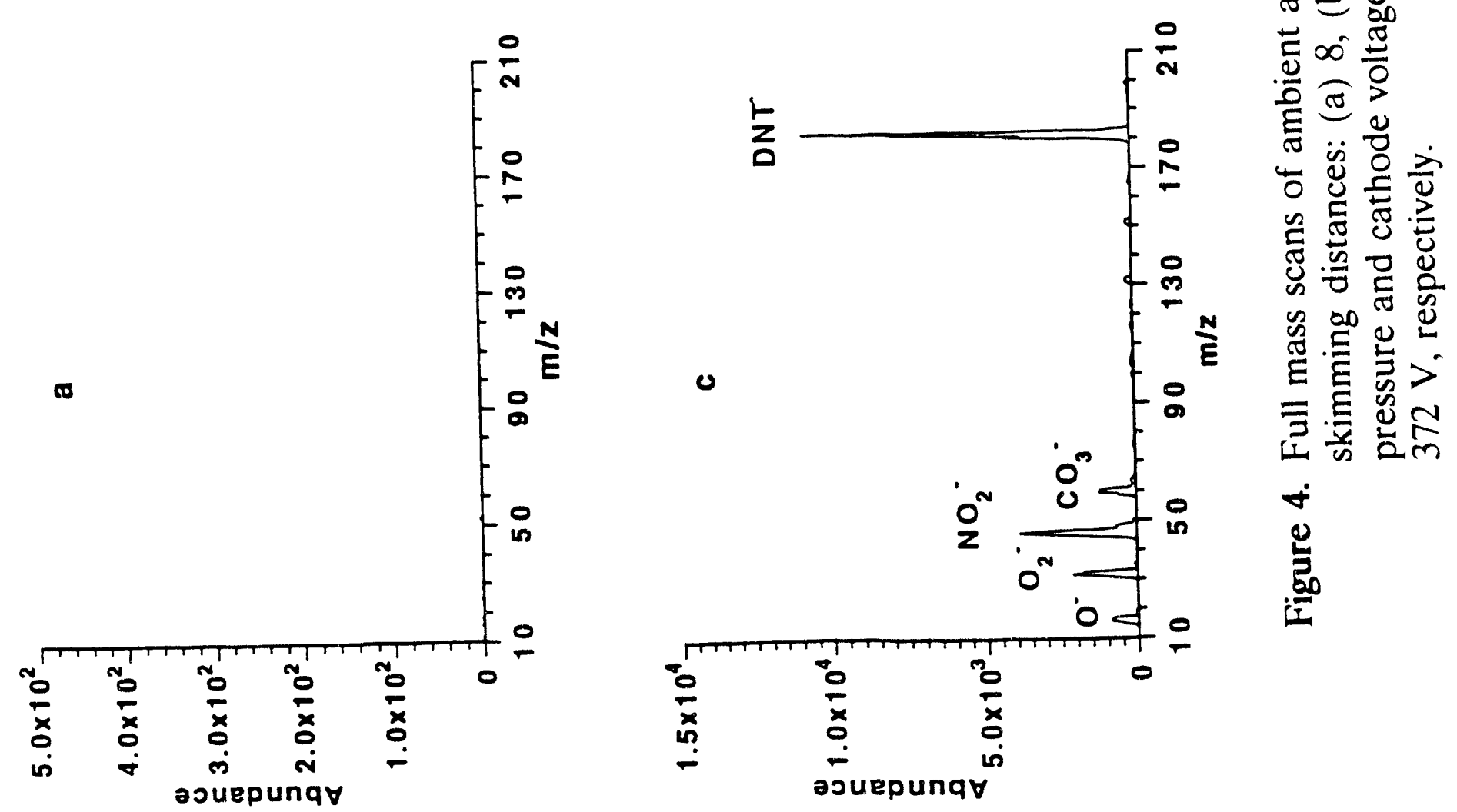
Figure 5 show both the minimum source pressure and skimming distance behind the Mach disk needed to observe the DNT anion signal. These latter values were obtained by subtracting the calculated Mach disk location from the skimming distance. The Mach disk location was calculated from the corresponding source pressure; for example, at a source pressure of approximately 0.75 Torr, the Mach disk is expected to form $4.3 \mathrm{~mm}$ downstream from the sampling inlet (Table I). At this source pressure a sampler-skimmer separation of at least $10 \mathrm{~mm}$ had to be maintained to observe DNT anions. The skimming distance behind the Mach disk is then $5.7 \mathrm{~mm}$. Skimmer separation from the Mach disk required to maintain a minimal level of detection was found to increase linearly, despite the fact that lower source pressures cause the Mach disk itself to form farther downstream. These findings confirm the previous results that anion formation is not occurring in the Mach disk and identify the need for a specific pressure requirement on the basis of anode-cathode separation. If skimming were required to occur from this particular zone to observe anions, then skimming distance behind the Mach disk should have remained relatively constant. Even though these findings suggest that anion formation might be independent of gas dynamics, experiments on lateral skimming across the expansion indicate that ion detection is dependent on mean molecular flow derived from the expansion.

Results derived in these studies also provide insight into ionization mechanisms for various species observed in the mass spectra. Electron/molecule and ion/molecule reactions as monitored by shifts in relative abundances of DNT and air-matrix ions were found to be influenced by changes in skimming distance and source pressure. Similar trends as those seen in Figure 4, where the skimming distance was varied at a fixed source pressure, were seen as the source pressure was varied and the skimming distance was fixed. The data plotted in Figure 6 were collected at a constant skimming distance of $12 \mathrm{~mm}$; however, because ionization increases with pressure [23] the cathode voltage was adjusted to maintain a constant cathode current of $-10 \mathrm{~mA}$. Results plotted in Figure 6 include ion intensities for DNT (m/z 182) O (m/z 16), O O $\mathrm{O}_{2} \quad(\mathrm{~m} / \mathrm{z} 32), \mathrm{NO}_{2}$ - 
Distance Behind

\section{Mach Disk (mm)}

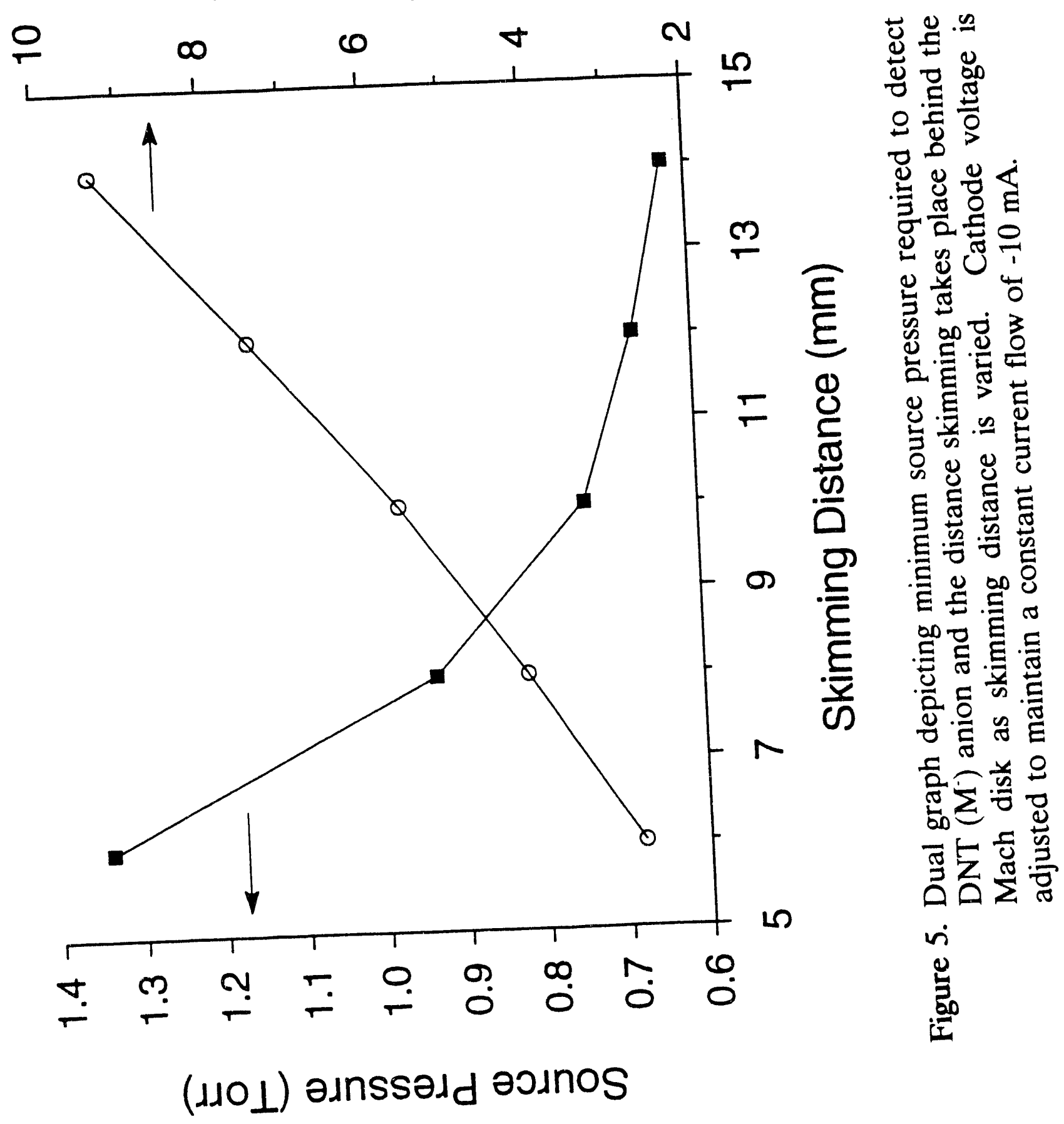




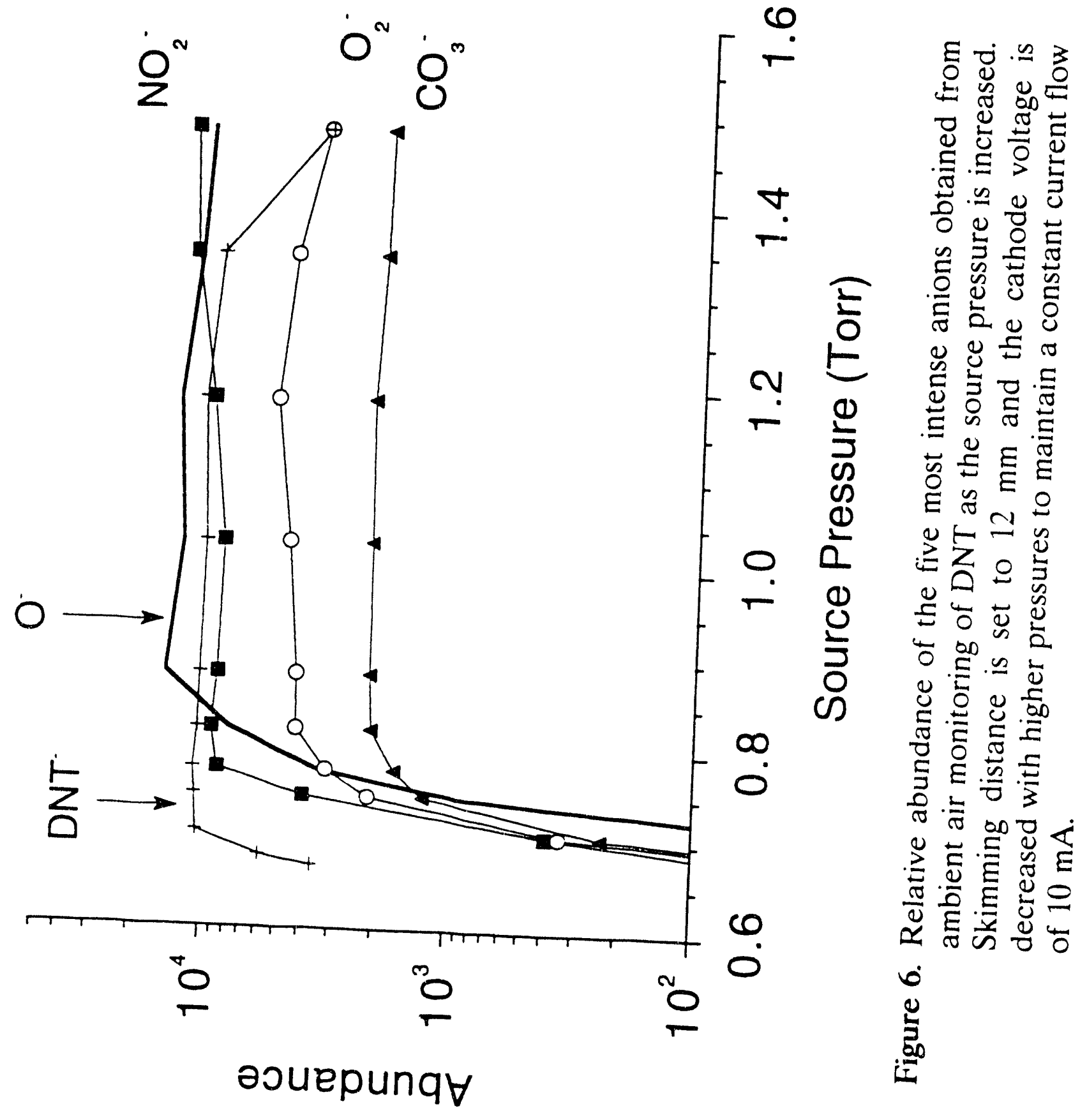


$(\mathrm{m} / \mathrm{z} 46)$, and $\mathrm{CO}_{3}{ }^{\circ}(\mathrm{m} / \mathrm{z} 60)$ as the source pressure was increased from 0.65 to 1.50 Torr. A gradual increase in the source pressure first raised and maximized the DNT anion signal at which point signals for $\mathrm{O}_{2}, \mathrm{NO}_{2}$, and $\mathrm{CO}_{3}$ rose together but at different rates. At a higher pressure, 0.75 Torr, the $\mathrm{O}$ signal begins to rise in intensity.

As indicated in the results presented in both Figures 4 and 6, DNT was primarily observed as the molecular anion. The absence of an $(\mathrm{M}-\mathrm{H})$ ion, a result of proton extraction, and only minor contributions from dissociative processes and cluster formation, limited the possible ionization processes to electron capture and charge transfer. Because source conditions that favor optimal DNT anion signals could be achieved without the presence of background species, electron capture is believed to be the primary means of ionization. Charge transfer is not believed to contribute significantly under such conditions as deduced from the absence of any reactant anions in the mass spectral background. Other evidence that supports the electron capture mechanism is that the DNT anion was formed in preference to more thermodynamically preferred products such as $\mathrm{CO}_{3}, \mathrm{NO}_{2}$, and $O$, which have higher electron affinities (see Table II) [24]. Under such conditions the DNT anion is believed to be kinetically favored undergoing relatively few ionmolecule collisions before entering into the mass analyzer chamber. As the skimming distance (Figure 4) or source pressure (Figure 6) was increased the more thermodynamically favored products became prominent as residence time or total number of collisions increase. One possible explanation for this phenomenon is that the gas density that exists near the jet boundary is sufficient to promote EC and subsequent stabilization through a third body collision. However, because there is an absence of $\mathrm{O}_{2}, \mathrm{NO}_{2}$, and $\mathrm{CO}_{3}$, which also require multiple [25] or third body collisions [26] for efficient formation, DNT anion production might be assisted further by inelastic collisions among the bath gas and electrons that can moderate their kinetic energy. An electron capture mechanism would account for the ultra-low detection limits reported for the ASGDI 
because the rate constants for electron capture can be up to two orders of magnitude greater than those of fast ion-molecule reactions [27].

\begin{tabular}{|c|c||}
\hline Table II. Electron Affinities for Major Species Observed in ASGDI Mass \\
Spectra
\end{tabular}

Experiments were also performed to study the effect of cathode voltage on the anion signal. The plot shown in Figure 7 was obtained at various cathode voltages while both skimming distance and source backing pressure were left unchanged at $12 \mathrm{~mm}$ and 1.49 Torr, respectively. This type of experiment provided a means to vary glow discharge processes while gas dynamic properties remained relatively unaffected. For example, as the cathode voltage was varied from -348 to $-365 \mathrm{~V}$, the cathode current increased from -10 to $-20 \mathrm{~mA}$ at a constant source pressure. This abrupt rise in cathode current is the result of greater cathode sputter rates that accompany higher energy and density of charged species. Measurements shown in Figure 7 were taken at a high source pressure so any effects on background and DNT anion signals could be monitored simultaneously. Nevertheless, relative signals among these species exhibited little change.

At lower source pressures and shorter skimming distances that precluded the formation of background ions, higher cathode voltages increased only the DNT anion signal. Shown in Figure 8 is a spectrum under such conditions with a $12-\mathrm{mm}$ skimming distance and 0.67 Torr source pressure. The cathode voltage was maximized to $-436 \mathrm{~V}$ to achieve the $-20 \mathrm{~mA}$ current limit for the power supply. 


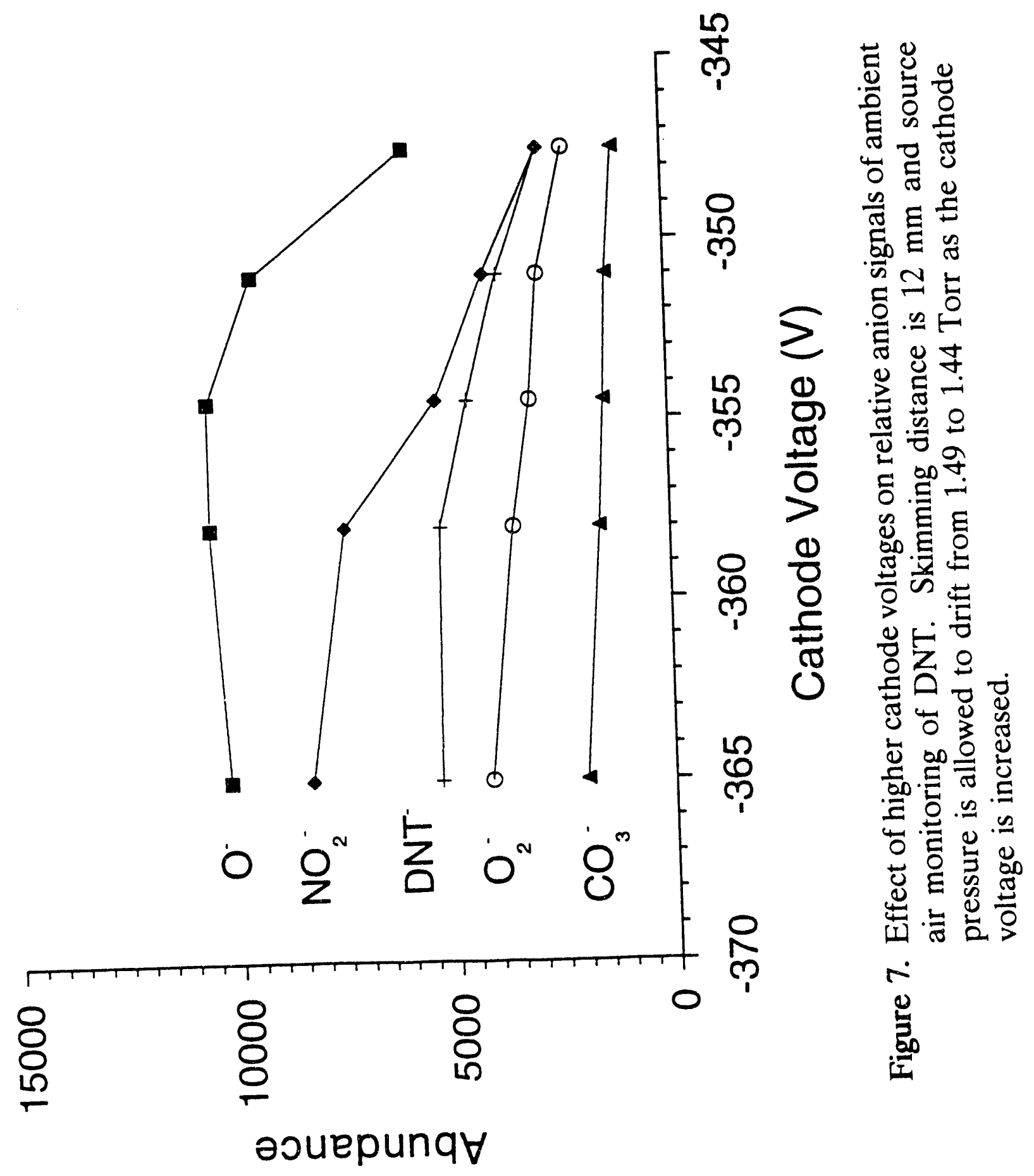




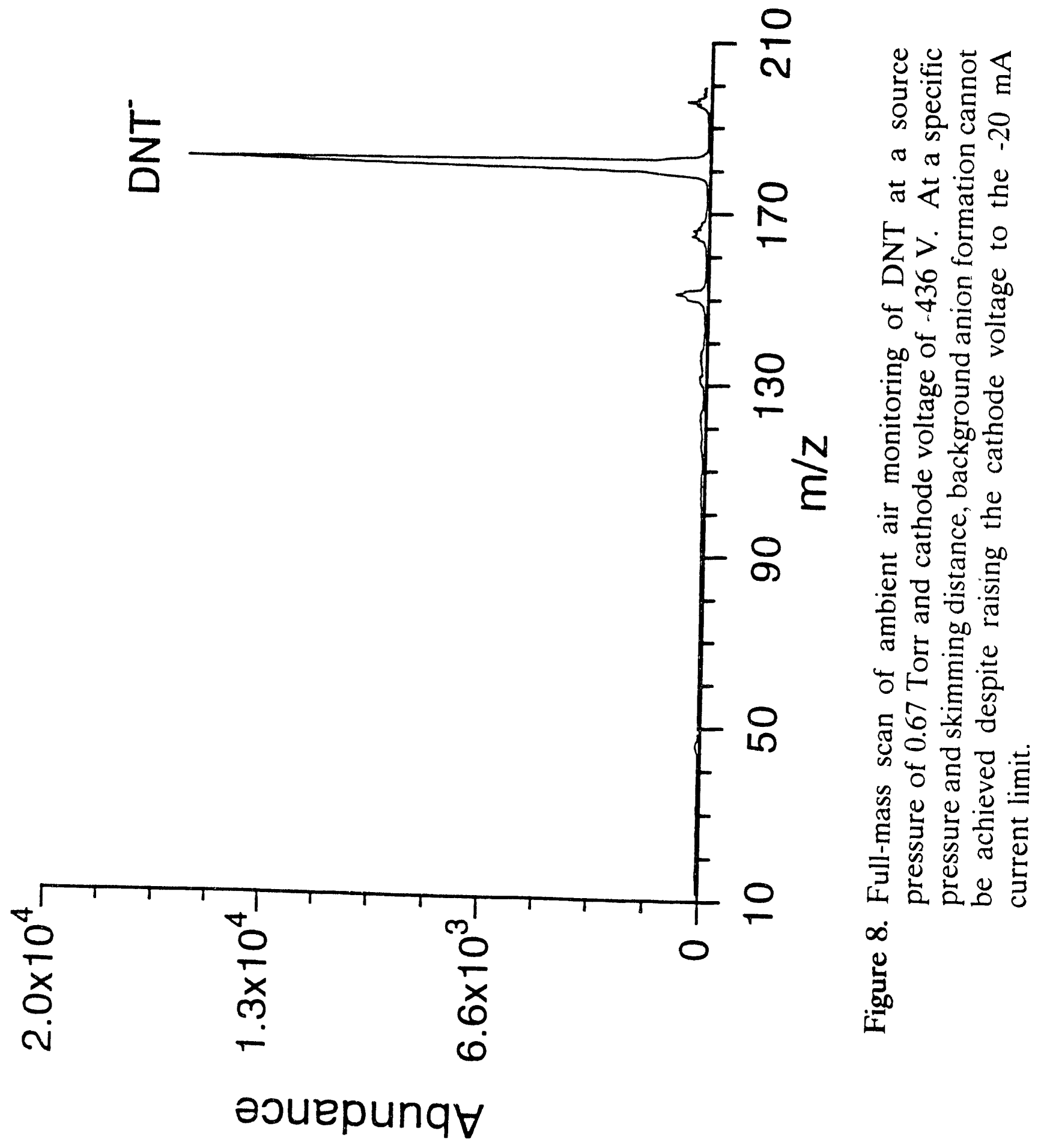


The role of the free-jet expansion in anion formation in ASGDI can be further studied by moving the two apertures off-line. This was effected by positioning the sampling orifice off-axis. This approach allowed us to maintain the separation and geometry of the ASGDI electrodes (i.e., sampling plate, coii and skimmer) while probing the various expansion rejions. In the results presented in Figures 9 and 10, ion signals for DNT (M) anion at two skimming positions, on-axis and $1 \mathrm{~mm}$ off-axis, are compared at two skimming distances, 7 and $12 \mathrm{~mm}$, respectively. At the 7-mm skimming distance (Figure 9) ion signals extended down to lower pressures as skimming was moved $1 \mathrm{~mm}$ off-axis. This response is significant because under similar conditions of source pressure and skimming distance, ion signals, which could not be observed while on-axis, became detectable when skimming was moved off-axis toward the reflected shock. The fact that anion formation is restricted directly behind the Mach disk might be related to the hydrodynamic flow of the expansion. For glow discharge sustaining species to penetrate on-axis toward the Mach disk they must either cross the reflected shock or originate upstream and travel parallel to the expansion. The reflected shock should more readily sustain glow discharge processes as sputtered ions and electrons from the cathode extend along the barrel shock boundary toward the skimmer cone. Those species that constitute the reflected shock maintain a portion of their directed flow and proceed toward the skimmer. As the source pressure is raised the distinguishable regions of the expansion become compressed upstream and cannot be discerned as a function of lateral skimming distance. This explanation accounts for the coinciding drop in ion signal with higher source pressures as pressure exceeds 1.0 Torr in Figure 9 and a similarity in the responses observed at the two axial positions, 12-mm downstream (Figure 10).

At skimming positions farther off-axis, from 3 to $12 \mathrm{~mm}$, both DNT and background ions were not detectable regardless of skimming distance, source pressure or cathode voltage. This response is believed to a result of skimming outside the jet boundary as deduced from the dimensions of the classical free-jet 


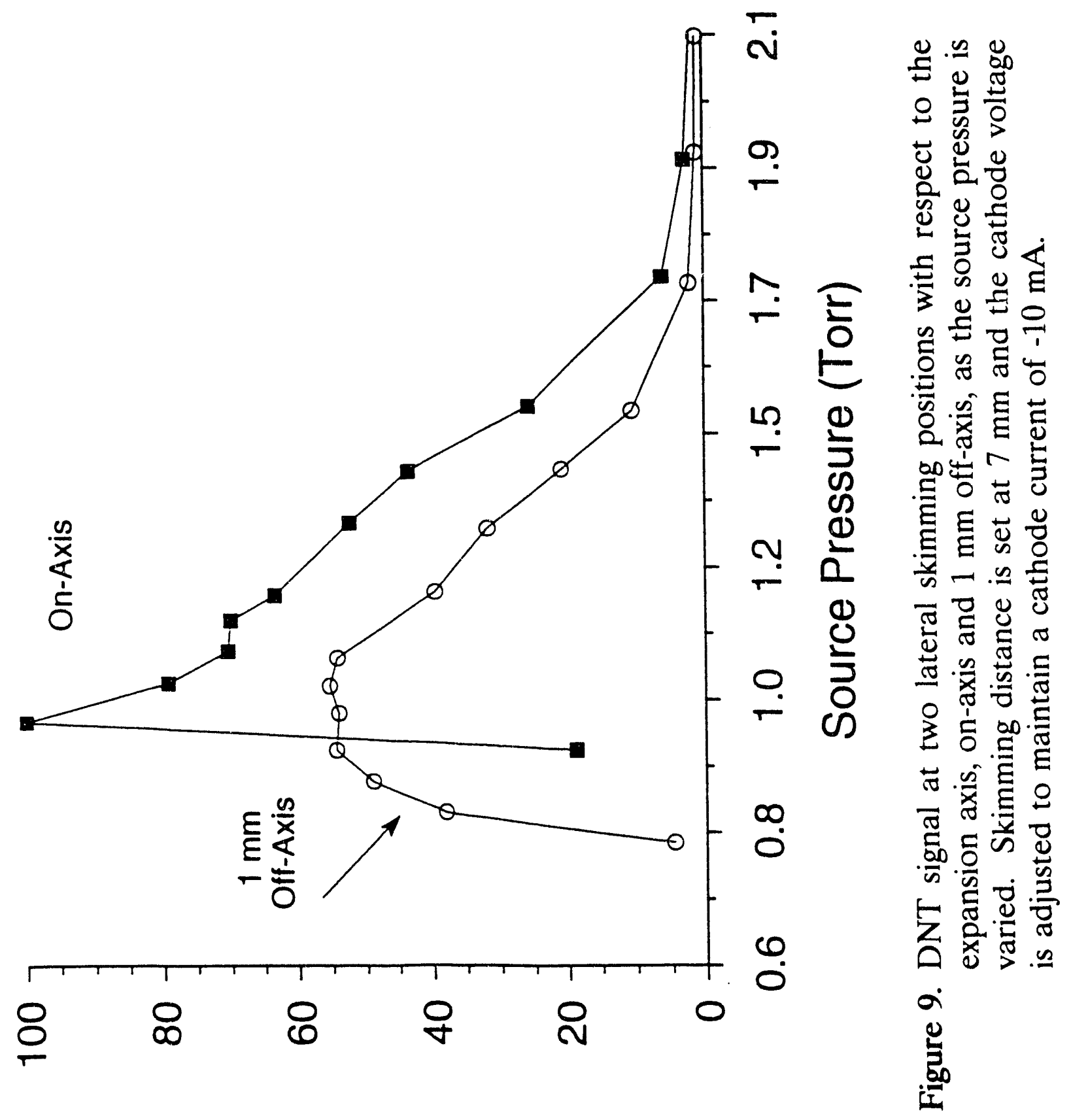

(\%) әouepunq $\forall$ әм!џе|әу 


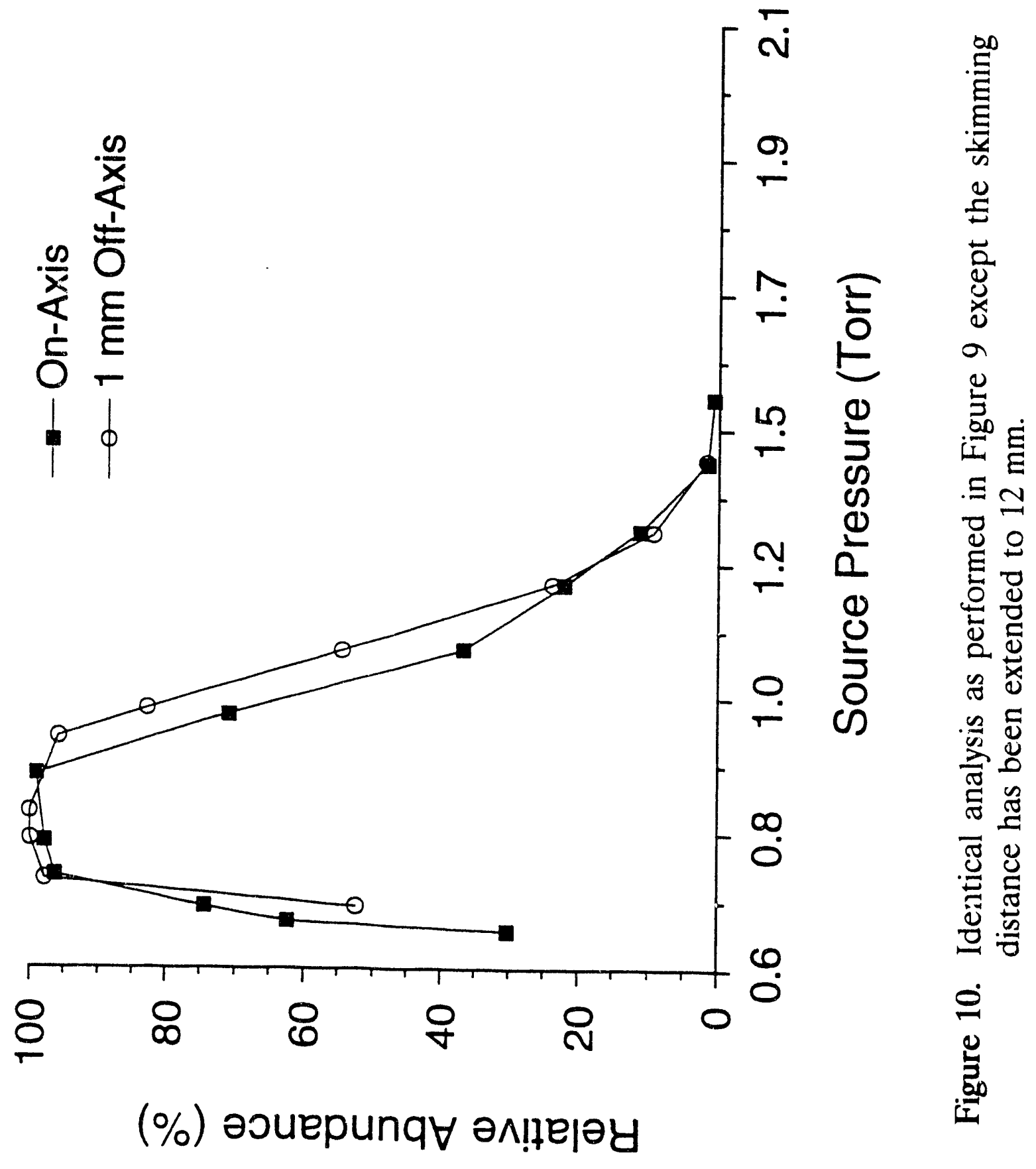


expansion (see Table 1). At the lowest obtainable source pressure of 0.48 Torr the jet boundary is predicted to form approximately $1.5 \mathrm{~mm}$ off-axis.

4. Conclusions Regarding the Role of Gas Dynamics in ASGDI The unique combination of hydrodynamic flow and glow discharge processes that exists in ASGDI produces an environment for efficient anion formation by EC. When ASGDI instrumental parameters are optimized, for example, either by mechanically adjusting the skimming position or by varying the source pressure, a specific ionization mechanism appears to dominate. In the case of DNT (M) anion formation the primary ionization mechanism is believed to be electron capture. The fact that we observe predominantly a molecular anion with very littie fragmentation suggests that collisional stabilization is occurring based on a report in which the analog TNT shows extensive fragmentation upon $0 \mathrm{eV}$ electron capture [8]. Regardless of why the molecular anion remains intact upon EC, the experimental data are indicative of relatively few collisions among gas species after ionization. This phenomenon was demonstrated for DNT where the $\mathrm{M}$ anion abundance was maximized without the formation of air-matrix species $\left(\mathrm{O}_{2}, \mathrm{NO}_{2}\right.$, and $\left.\mathrm{CO}_{3}\right)$ that require multiple or third-body collisions for formation. Under such conditions where the number of collisions is not suitable to promote ion-molecule reactions among background species, collisional frequency might be sufficient to moderate electron energies. Understandably, the flow dynamics of the expansion also contribute to this ionization process. It was shown that skimming is limited to the region behind the jet boundary whereby moving the skimmer a few millimeters off-axis resulted in a complete loss of ion signal. This dependency is believed to be an effect of mean molecular flow that influences residence times and thus can be manipulated to favor observation of kinetically fast reactions over more thermodynamically preferred processes. 


\section{IV.TANDEM MASS SPECTROMETRY}

Single stage mass spectrometry is one of the most sensitive and specific instrumental methods available to identify organic compounds. However, it cannot perform trace analysis of complex mixtures without an extremely selective ionization method and/or some prior separation step. The most common technique involving mass spectrometry for the analysis of mixtures of volatile compounds is the combination of gas chromatography with mass spectrometry (GC/MS;. Historically, gas chromatography has not played an important role in real-time rnixture analysis due to the relatively long times required for separation. (Recently, developinents in fast GC have made its use in explosives vapor detection a viable pirt of the analysis.) An alternative to GC/MS in targeted compound analysis is to replace the GC with a mass spectrometer to perform inass spectrometry/mass spectrometry (MS/MS), or taridem mass spectrometry $[28,29]$. Since the mass spectrometer selects according to mass/charge, mixture components can be analyzed in any sequence, which is an advantage when cily a few components are of interest. Furthermore, the first stage mass-selection step can take place on the time-frame of microseconds to milliseconds, depending on the mass spectrometry technology (see below). Tris time-frame obviously compares favorably with chromatography. A schematic comparison of GC/MS with MS/MS is shown in Figure 11 assuming an ionization method that produces positive ions.

A chemical reaction resulting in a change in mass/charge must occur between stages of mass spectrometry in an MS/MS experiment for the second stage of mass analysis to krovide new information. The most common reaction is fragmentation shown generically below for a molecular anion:

$$
m_{1} \cdot \cdots>m_{2}+m_{3}
$$

where $m_{1}$ represents an excited molecular anion and $m_{2}$ and $m_{3}$ represent the charged and neutral products of a dissociation reaction, respectively. The dissociation of a so-called "parent" ion into product ions and neutrals is ordinarily 


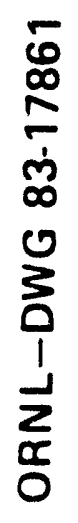

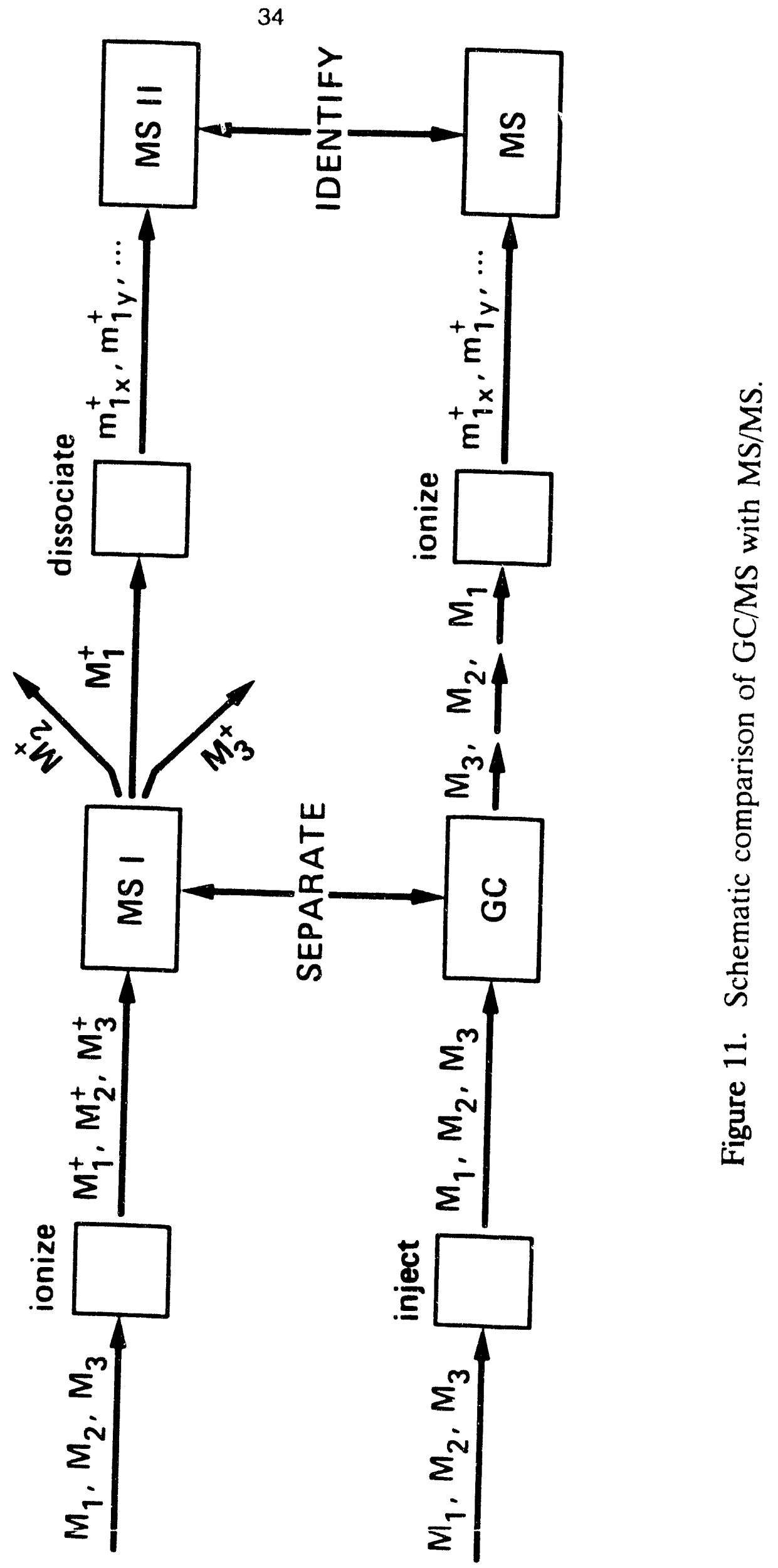


induced via one or more energetic collisions of the parent ions with neutral species admitted ii to the instrument to serve as collision partners. This process involving the production of excited parent ions which subsequently dissociate into products is commonly referred to as collision-induced dissociation (CID) [30]. Therefore, the identification of a targeted compound in a typical MS/MS experiment is based on the mass/charge ratios of the parent and product ions. Tandem mass spectrometry has a variety of important applications. Among the most widely recognized is direct targeted compound analysis. Explcsives vapor detection is, of course, a targeted compound analysis problem. The MS/MS experiment for explosives detection is shown schematically in Figure 12 as proceeding from left to right. Air is drawn into the glow discharge source where explosives are converted to negative ions. The anions that issue from the ASGDI source are subjected to a stage of mass analysis. If ions of mass/charge corresponding to those expected for a targeted explosive are present, they are subjected to collisional activation. The product ions formed from CID are then subjected to a second stage of mass analysis. A molecule to be identified as an explosive must pass three tests. First, it must form a negative ion. That is, it must be ionized by the ASGDI in the selective negative ionization mode. Second, it must form an ion of mass/charge expected for a targeted explosive. And third, it must form the product ion(s) expected for the explosive ion of the selected parent ion mass/charge. These three tests constitute an extremely specific identification procedure designed to minimize false alarms.

It is difficult to predict a priori the false alarm rate for any of the current explosives detectors in a real-world scenario since the complexity of the mixtures to be confronted is poorly characterized at best. However, it is possible to compare the techniques on the basis of the number of resolution elements in the analysis [31]. The number of resolution elements determines, in part, the informing power of an analytical technique. Informing power correlates with specificity. As a rule, as a mixture becomes more complex, greater informing power is required to distinguish between mixture components. A typical IMS spectrum is $\approx 20 \mathrm{~ms}$ 


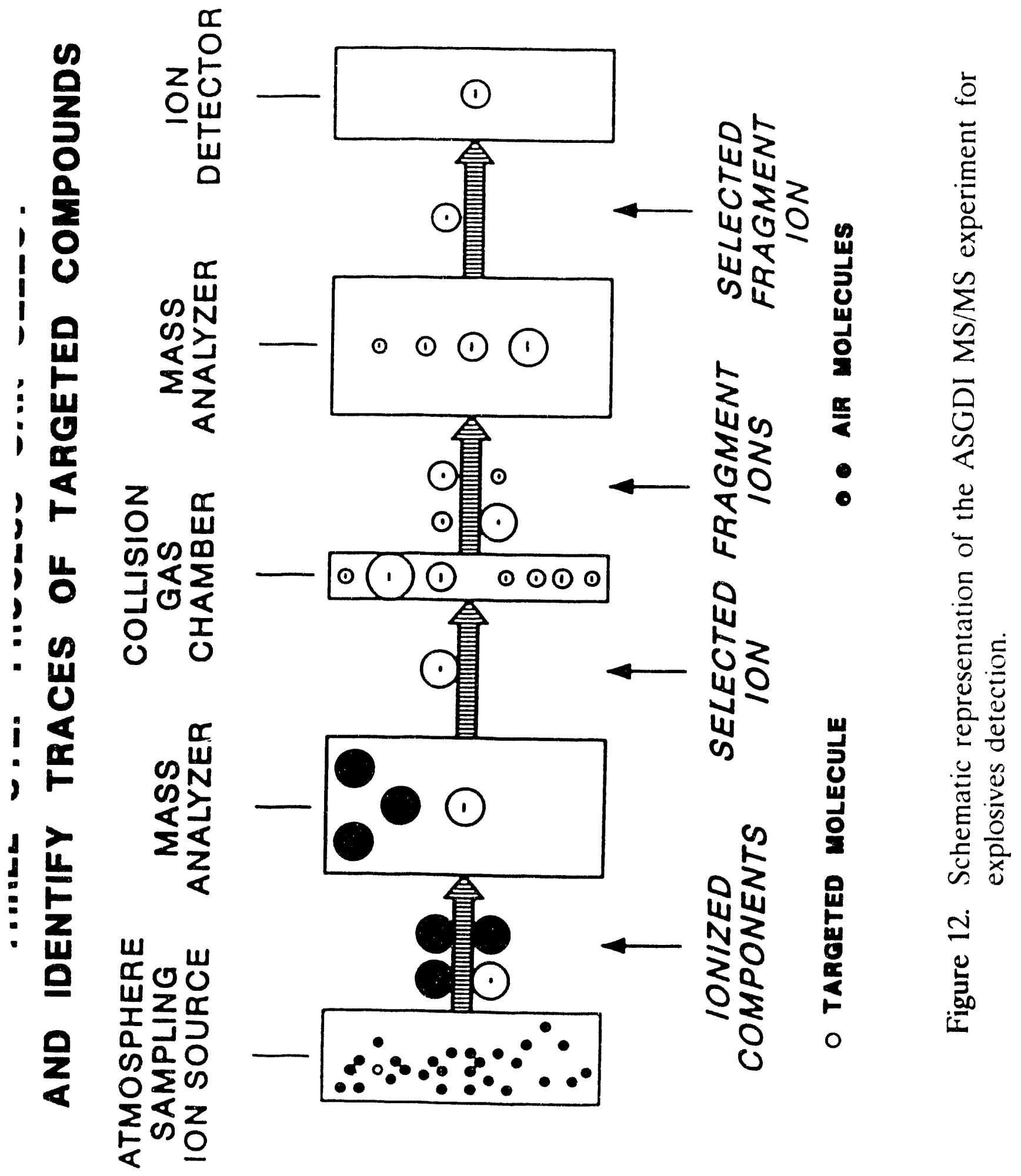


wide and a typical peak width at half height is $1 \mathrm{~ms}$. There are, therefore, $\approx 20$ resolution elements for the ion mobility spectrometer. The overall specificity of the analysis relies heavily on the selectivity afforded by negative ion API. Similarly, for $\mathrm{GC} / E C D$, the resolving power of the $\mathrm{GC}$ analysis is determined by the chromatographic resolution. For most gas chromatographs used in explosives detection there are, at best, a few hundred resolution elements in the typical chromatogram. The overall specificity of the analysis is enhanced by ECD. In MS/MS, the number of resolution elements is the product of the resolution elements of each stage of MS. For most tandem mass spectrometers based on quadrupole technology, the resolution of each stage of MS is typically 1000 (unit mass resolution throughout the mass range). The explosives ions generally fall below $\mathrm{m} / \mathrm{z} 300$ so that, realistically, there are about 300 useful resolution elements/stage of MS. The number of resolution elements for MS/MS is therefore $\approx 90,000$. This number may be increased if additional resolution elements are added by varying the parameters that affect the reaction between stages of MS [8]. The specificity of the overall analysis is, of course, enhanced by analyzing negative ions. Informing power is also determined by the dynamic range of the analysis [8] which further increases the informing power of MS/MS over the IMS and ECD. The informing power of MS/MS is therefore several orders of magnitude greater than any of the other commonly employed vapor detectors.

The sensitivity of an explosives detector employing MS/MS is determined in part by the throughput of the MS/MS instrument. This involves the efficiency of the first stage of MS, the efficiency with which fragment ions are produced from the parent ions, the efficiency of the second stage of MS, and the detection efficiency. These numbers are determined by the mass analyzers, ion optics, and the way in which CID is effected. There have been a wide variety of instrumental approaches to MS/MS. Each has its own set of performance characteristics and many have unique advantages for particular applications. There is no "best" instrumental approach for all MS/MS experiments. However, the number of approaches suitable for fieldable explosives detection is relatively limited. A short 
discussion of the various approaches to MS/MS is given in the following sections with particular emphasis on the desirable qualities for a tandem mass spectrometer in the explosives detection scenario. These approaches can be conveniently classified as either beam-type technologies or ion trapping technologies.

\section{A. Beam-type Technologies}

The term "beam-type" instrument refers to a mass spectrometer in which ions formed in an ion source are extracted and accelerated, usually continuously thereby forming an ion beam, through static magnetic and electric fields or through electrodynamic fields wherein the trajectories of the ions are altered in a mass/charge dependent fashion. The mass/charge discrimination, therefore, occurs in space. In the case of a magnetic sector mass spectrometer, for example, the radii of deflection of ions in a monoenergetic ion beam are determined by the momentum/charge ratios of the ions. A slit or aperture at the focal point of the magnetic sector allows a limited range of momentum/charge ratios to pass to a collision region, for example. In the case of a quadrupole mass filter, the radio-frequency and fixed voltages applied to the four rods comprising the device can be established to provide for high pass filtering in one dimension and low pass filtering in the other such that only a limited range of mass/charge ratios can pass through the device. In the case of the time-of-flight mass spectrometer, a monoenergetic "packet" of ions is separated according to transit times. The term "beam-type" tandem mass spectrometer is intended to imply further that the stages of mass spectrometry and collision-induced dissociation occur in discrete regions of the spectrometer $[28,29]$.

Some of the more commonly employed beam-type tandem mass spectrometers are shown schematically in Figure 13 where $S$ is the ion source, $C$ is a collision cell, D is a detector, B is a magnetic sector, $E$ is an electrostatic analyzer, and $\mathrm{Q}$ is a quadrupole mass filter. The first analytical tandem mass spectrometers were two sector instruments of either "forward" geometry (EB) or "reversed" (BE) geometry. In the former, CID is effected between the ion source 
ORNL-DWG 83-19716

\section{INDIVIDUAL ANALYZERS CAN BE COUPLED SEVERAL WAYS FOR MS/MS}
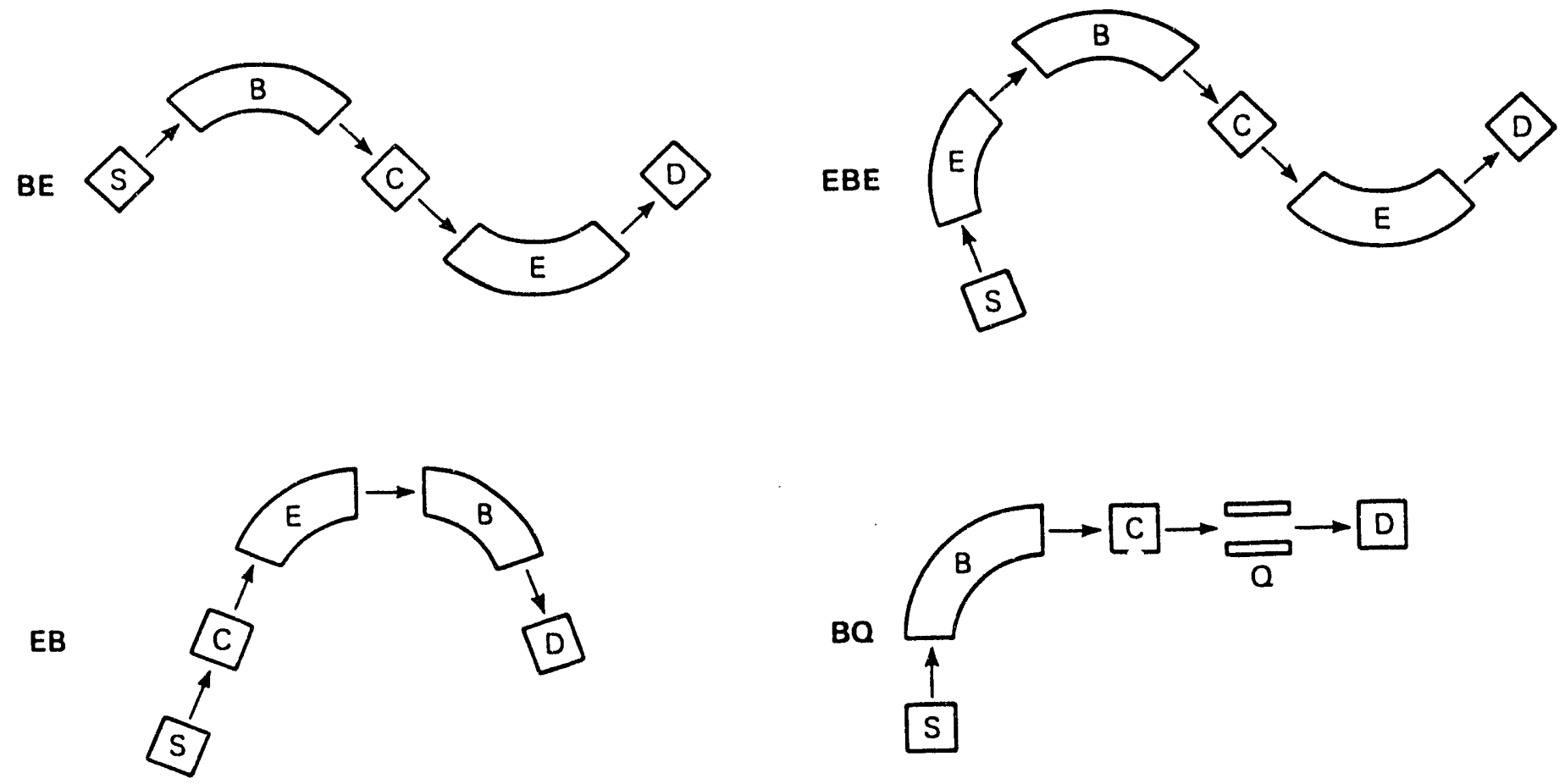

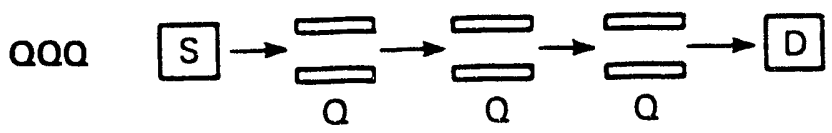

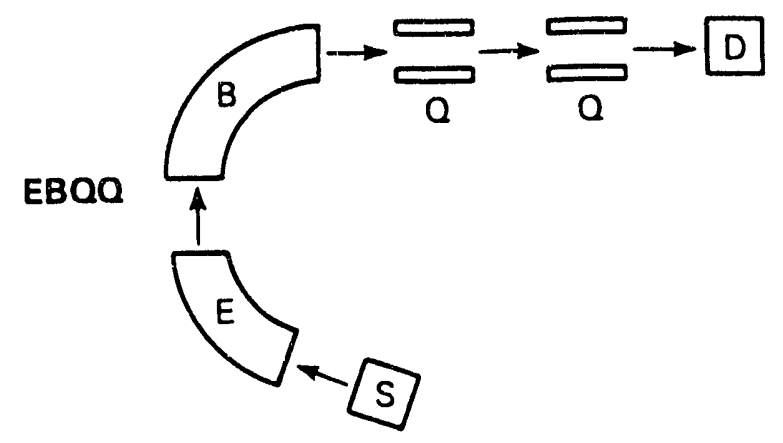

Figure 13. Some common MS/MS instrumental configurations. 
and the electrostatic analyzer and product ions are analyzed by scanning the electric and magnetic fields simultaneously and at a fixed ratio. In the latter, CID can also be effected between the ion source and the first sector but more usually is effected between sectors. In the latter case, product ions are analyzed by scanning the electric sector. More elaborate multi-sector instruments have since been constructed primarily for research purposes, as opposed to routine analytical research.

The multiple quadrupole tandem mass spectrometers were introduced primarily for analytical applications $[32,33]$. There are many important distinctions between the multi-sector and multi-quadrupole systems. A full discussion of these is beyond the scope of this overview. However, a few important distinctions are that quadrupole mass filters are readily programmable, mass resolution/transmission is variable via software over a range, and they lend themselves much more readily to field transportability. Therefore, for the reasons of size, weight, and cost alone, multiple quadrupole instruments are the most appropriate of the commercially available beam-type tandem mass spectrometers to consider for explosives detection. It is important to note that while commercial instruments are often referred to as "triple quadrupole mass spectrometers", only the first and third quadrupoles are used as mass analyzers. The central quadrupole is operated in the high pass filter (non-analyzing) mode. In fact, in many of the modern "triple quadrupole" instruments, the collision cell is actually an octapole. This is the case in the Finnigan TSQ700, the instrument modified for explosives detection as part of this interagency agreement.

Figure 13 also shows several instrument geometries that combine sectors and quadrupoles. These instruments are referred to collectively as "hybrid" mass spectrometers [34-36]. These combinations are not inherently compatible as are the $\mathrm{EB}$ and $\mathrm{BE}$ geometries because quadrupoles operate best on ions with kinetic energies of tens of electronvolts at most, whereas sectors typically operate on ions with kiloelectronvolt kinetic energies. Therefore, one or more acceleration/deceleration steps must be effected in hybrids. Nevertheless, the 
combined strengths of these analyzers in a single instrument provide for unique research capabilities. However, from the point of view of explosives detection, hybrids have not been pursued for the same reasons that multi-sector instruments have not. For the purpose of comparing and contrasting beam-type tandem mass spectrometers with trapping instruments described in the next section, a number of characteristics are listed here which apply primarily to the multiple quadrupole instruments. As discussed further in this report, and in some cases illustrated with direct experimental comparisons, beam-type and trapping technologies each enjoy advantages as the tandem mass spectrometer for explosives detection. However, since the beginning of this agreement, the rapid development of the quadrupole ion trap has shifted the balance strongly in favor of the trapping technology for explosives detection in the field. Important characteristics of beam-type technologies relevant to this discussion include:

Sensitivity - While sub-picogram detection limits for explosives can be readily achieved in the MS mode in the absence of an isobaric interference, such low detection limits are challenging in the MS/MS mode due to transmission losses. Beam-type instruments usually provide many opportunities for ion losses during the passage of ions from the ion source to the detector. Fringing fields at the entrance and exit of each multipole can lead to ion loss, for example. There is also the usual compromise between resolution and transmission with the mass analyzers. Scattering of parent ions and product ions in the collision region can lead to significant losses as well. Indeed, the MS/MS efficiency, defined as the number of product ions measured in the MS/MS experiment divided by the number of parent ions measured prior to admitting the collision gas, is typically a few percent at best. Therefore, there is a significant cost in analytical signal in going from MS to MS/MS. (However, this is sometimes more than offset by the reduction in chemical noise arising from isobaric interferences.) 
- Specificity - As mentioned above, specificity is determined by the number of non-redundant resolution elements in the analysis. The mass resolving power of each mass analyzer can easily be 1000 with good transmission. Furthermore, the MS/MS spectrum of a polyatomic ion is often highly dependent upon collision energy and target gas pressure. This dependence can be used to provide more informing power if needed. From our experience, the specificity of the triple quadrupole instrument is sufficiently high without recourse to collision energy-resolved analysis. We have yet to find any interferences that might lead to false alarms that cannot be avoided with the standard MS/MS procedure.

- Speed - Ion flight times are on the order of tens of microseconds. For healthy signals, therefore, the response is essentially instantaneous. Even in cases where signal averaging can improve detectability, the mass spectrometer analysis time will be short relative to sampling and preconcentration steps.

- Size - Triple quadrupole instruments have not generally been engineered for field use although there is substantial room for miniaturization. However, the extensive pumping required for maintaining the analyzer quadrupoles at less than $10^{-5}$ Torr while the collision region is held at $1-5$ mTorr remains a limiting factor in size and weight considerations.

- Cost - Commercial triple quadrupole systems typically cost over $\$ 300 \mathrm{k}$. A system designed specifically for explosives detection might be made for less but probably not without a large market. Power supplies for three multipoles are required, machining for high performance quadrupoles is demanding, and pumping is extensive. These factors make the prospects for a low cost $(<\$ 100 \mathrm{~K})$ explosives detector seem remote considering the market size. 
- Dynamic range - Dynamic range refers to the range of analyte quantity or concentration over which the analytical signal is linear. The dynamic range of a mass filter is roughly $10^{8}$. We have observed the dynamic range of a quadrupole system coupled with an ASGDI source to be at least $10^{5}-10^{6}$. Dynamic range is a strength of beam-type technologies because ion-ion interactions that can affect linearity are not important at the beam currents typically created by the ASGDI source or most other ion sources used in analytical chemistry.

- Scan Flexibility - The ready software control of the multiple quadrupole instrument and the nature of the beam-type experiment allow for a variety of different scanning modes for screening. The conventional MS/MS experiment, the product ion scan, is but one. Others include the parent ion scan, the neutral loss scan, and the targeted product scan [37]. The latter is discussed further in a later section.

\section{B. Ion Trapping Technologies}

Several instrumental approaches to trapping charged particles and ions have been developed for research in physics and chemistry. Instruments used for analytical chemistry and which have been demonstrated to be capable of performing multiple stages of mass spectrometry include the ion cyclotron resonance (ICR) mass spectrometer [38] and the ion trap mass spectrometer [39]. The former is based on a static magnetic field and a perpendicular static electric field. The detection technique typically employed with commercial versions of the :CR involves image current measurement of all ions simultaneously and Fourier transformation of the time domain image current signal into the frequency domain. These instruments are usually referred to as Fourier transform mass spectrometers (FTMS) [40-44]. The FTMS technology has provided the highest mass resolving powers of any form of mass spectrometry. It is finding increasingly wide 
application in high performance mass spectrometry research and development. However, commercial systems employ relatively large superconducting magnets and must operate at low pressures for high resolution. Little effort has thus far been expended in making fieldable FTMS instrumentation although such a development is not precluded at some sacrifice in performance.

In contrast to the development of FTMS, the analytical development of the quadrupole ion trap, or Paul trap [45-51], has primarily focussed on analytical applications requiring moderate resolving powers and those in which relatively high background pressures exist. Work has proceeded at ORNL for several years in developing the combination of ASGDI with a quadrupole ion trap for explosives vapor detection. Advances in this area now lead us to believe that this form of ion trapping technology should be given serious consideration for real-world security applications. For this reason, this form of ion trapping mass spectrometer is discussed at more length below and elsewhere in this report.

The quadrupole ion trap employs three electrodes as shown in the photograph of Figure 14. Two end-cap electrodes sandwich a ring electrode. The position coordinates in the ion trap can be described by the cartesian coordinates $x, y$, and $z$. However, due to the symmetry around the ring electrode, it is convenient to indicate the $x$ and $y$ coordinates by $r$, the radial coordinate, where $r=\left(x^{2}+y^{2}\right)^{1 / 2}$. By convention, $z$ is referred to as the axial coordinate. Note that the holes in the end-caps allow for ions to enter or exit the ion trap. The end-caps are ordinarily held at ground potential while a radio-frequency ( $\mathrm{rf}$ ) signal is applied to the ring electrode. The hyperbolic shapes of the electrodes result in a quadrupole field within the ion trap cavity. A positive voltage on the ring electrode attracts negative ions and repels positive ions whereas a negative voltage on the ring electrode does just the opposite. The electric fields experienced by an ion for a large positive voltage and for a large negative voltage are illustrated in Figure 15. Note that for any non-zero voltage applied to the ring electrode, an ion finds itself in a potential well in one dimension and on a potential hill in the other. The key to trapping the ion is to vary the potential on the ring electrode at a frequency 


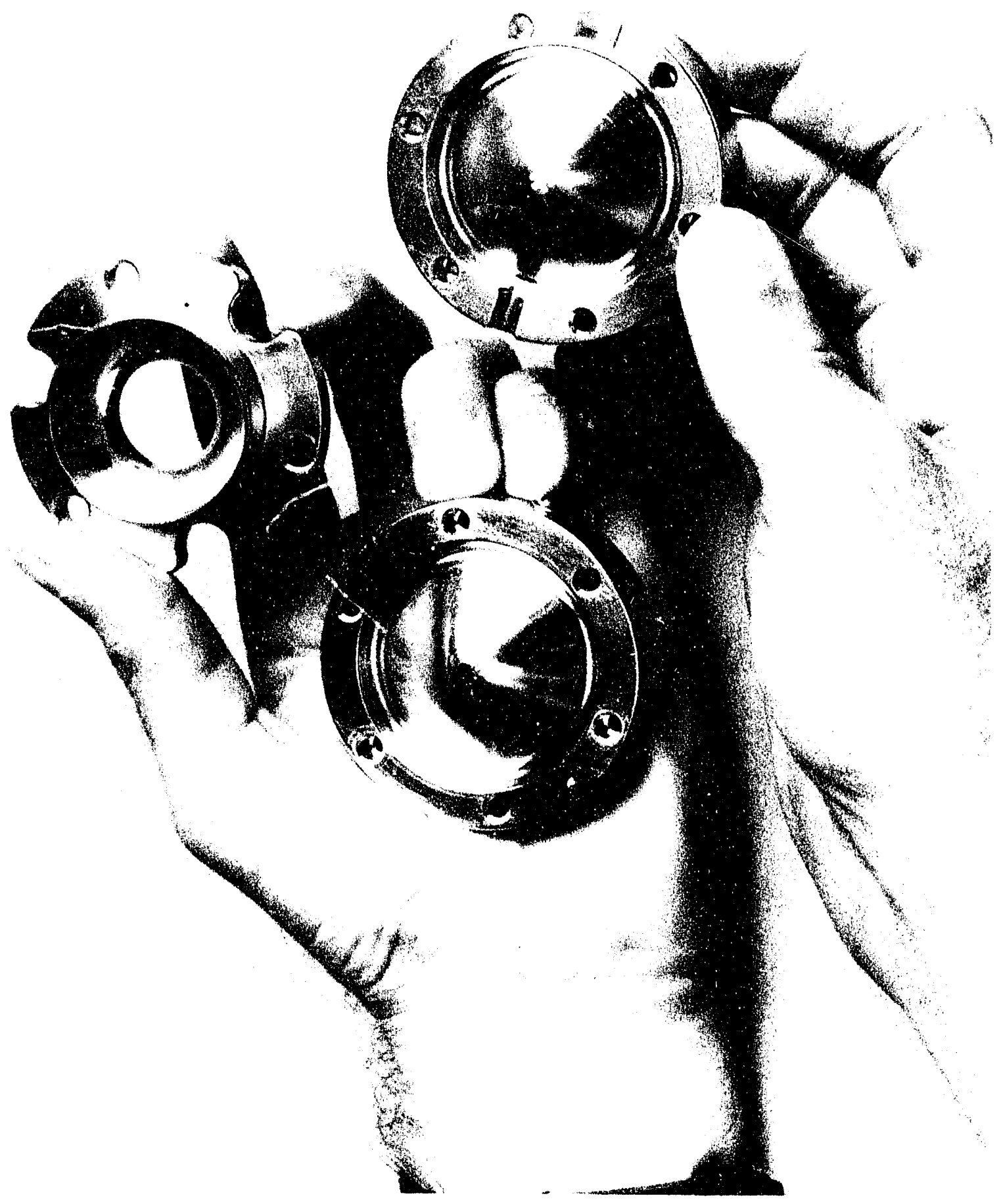

Figure 14. Photograph of the quadrupole ion trap assembly; two endcap electrodes and a ring electrode. 


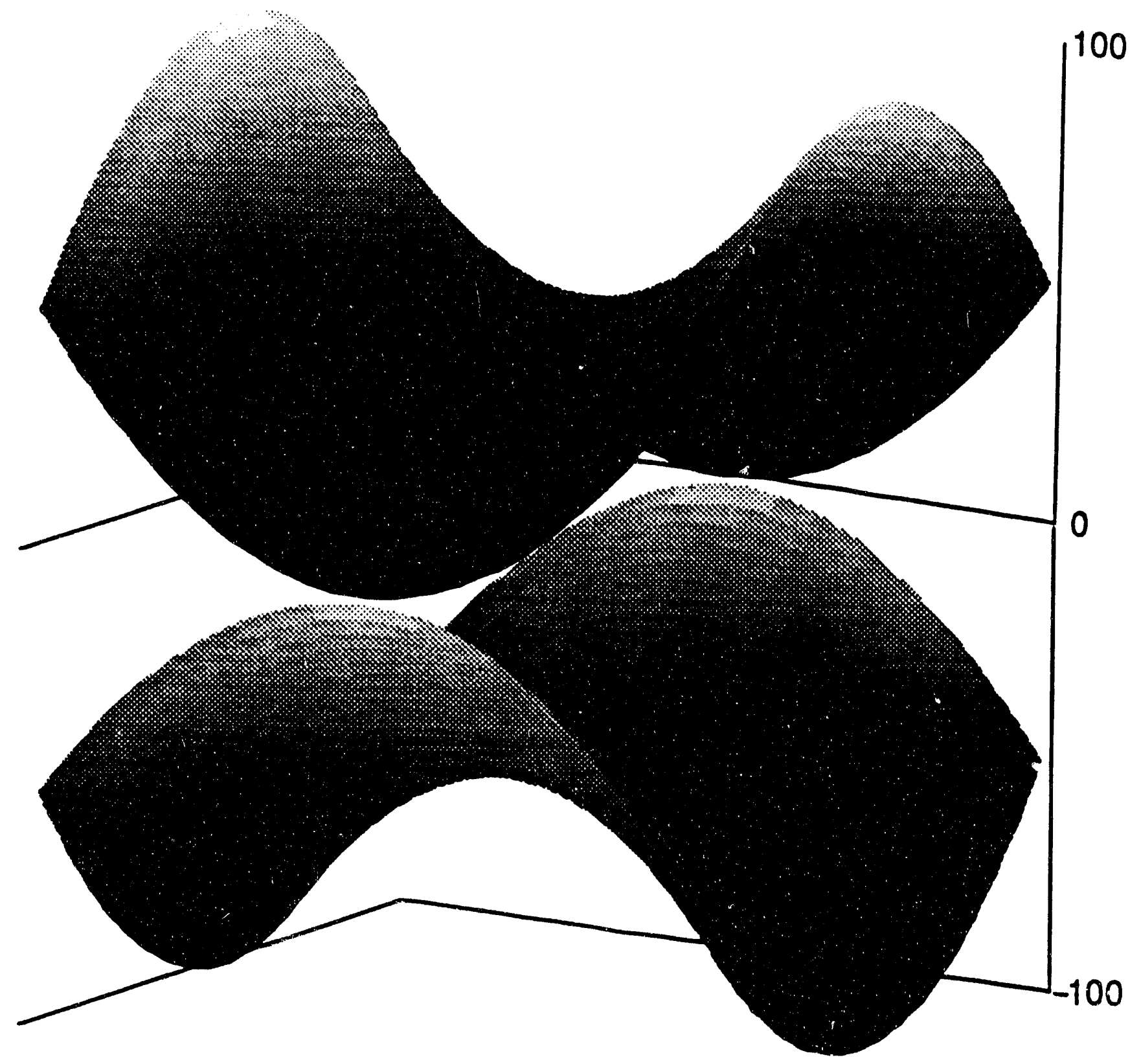

Figure 15. Schematic representation of the electric fields experienced by an ion for a large positive voltage and for a large negative voltage. 
sufficient to prevent the ion from experiencing a repulsive field long enough to exit the ion trap. The application of a sine wave to the ring electrode creates the effect of flipping the saddle-shaped fields of Figure 15 back and forth to hold ions in the middle of the saddle. Ion storage as well as capture of injected ions is facilitated by adding a light background gas, such as helium, to remove kinetic energy from the ions withoul scattering them away from the center of the ion trap.

Trapped ions can be mass-analyzed in a variety of ways. The most commonly employed methods involve ejecting ions from the ion trap and into an externally-mounted detector in a mass-dependent fashion. The so-called massselective instability scan introduced by Stafford et al. $[52,53]$, for example, involves scanning the amplitude of the if signal applied to the ring electrode causing ions to achieve unstable trajectories in the axial dimension sequentially from low mass to high mass. A variation on this approach is the "resonance ejection" scan [50]. For a given ring electrode if amplitude and frequency, ions of each mass/charge have characteristic frequencies in the axiai and radial dimensions. By scanning the ring electrode amplitude (or frequency) ions can be brought into resonance in the axial dimension with an alternating voltage applied to the end-caps. The application of a sinusoidal signal to the end-caps whi,e the normal trapping if signal is applied to the ring electrode is shown schema tically in Figure 16. As the ions come into resonance they may absorb sufficient fower to be ejected from the ion trap and into an external detector.

Just as there are a variety of ways to mass-anilyze ions with an ion trap, there are a variety of means for mass selecting parent ions for an MS/MS experiment. A commonly employed method is to add a dc voltage to the if signal applied to the ring electrode for mass selection. This is analogous to the method used for mass analysis and mass selection with the quadrupole mass filter. It effectively combines high pass filtering with low pass filtering to create a notch filter. Low mass ions are ejected in the axial dimension while high mass ions are destabilized in the radial dimension. Resonance ejection is also often used for parent ion isolation [54]. The amplitude of the ring electrode if signal is raised to 

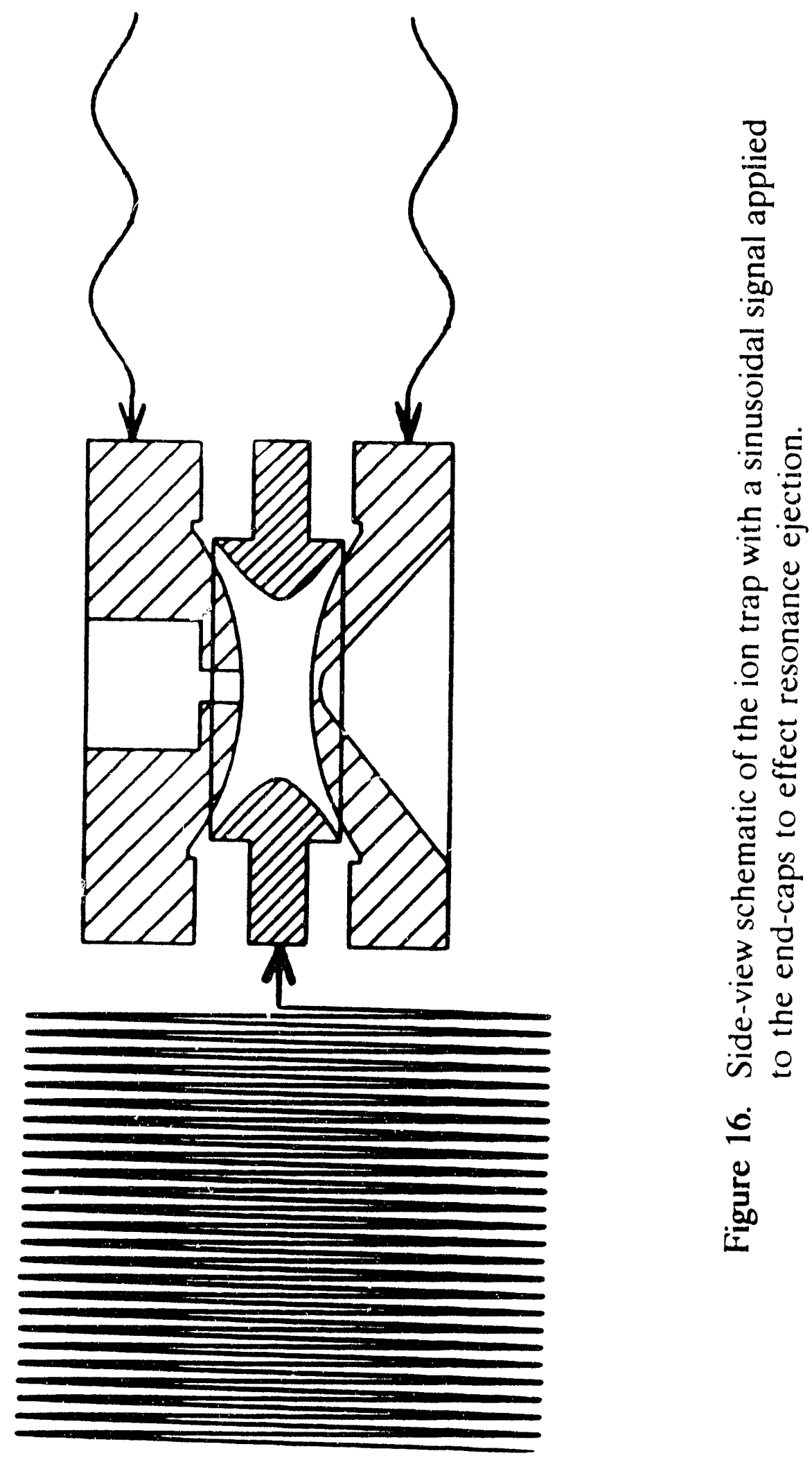
reject low mass ions while frequency of the signal applied to the end-caps is chosen to sweep out ions of higher mass than that of the parent ions. Newer techniques involve the application of a range of frequencies to the end-caps to allow for the accumulation of selected ions during ion injection. For the purpose of explosives detection, these mass selection methods are quite satisfactory.

Collisional activation is used to dissociate ions in the typical ion trap MS/MS experiment, just as it is with beam-type tandem mass spectrometers. However, the process is quite different [55]. It is beyond the scope of this report to describe all of the differences and their ramifications for studying gas-phase ions. However, to understand why ion trap collisional activation can lead to high sensitivity MS/MS it is important to recognize that collisional activation in the ion trap is a relatively slow heating process which allows for complete dissociation of a parent ion population. Furthermore, since there are no transmission losses associated with ion transport in an ion trapping instrument, the product ions can often be collected with $100 \%$ efficiency. Collisional activation is typically effected using "resonance excitation" as opposed to "resonance ejection". The only difference is that the amplitude of the excitation signal applied to the end-caps is insufficient for ion ejection. The presence of helium in the ion trap at a pressure of about one millitorr provides a damping force and a means for dissipation of the energy ions absorb from the field. The ions are heated internally during the course of resonance excitation and may be heated sufficiently to fragment. MS/MS efficiencies can be as high as $100 \%$ in favorable cases. An example is shown in Figure 17 which shows the isolated parent ion used for the detection of PETN, $\left(\mathrm{M}-\mathrm{CH}_{2} \mathrm{ONO}_{2}\right)$, at $\mathrm{m} / \mathrm{z} 240$ before resonance excitation and the spectrum acquired after resonance excitation. The product ion at $\mathrm{m} / \mathrm{z} 62$ is formed yielding an MS/MS efficiency of $100 \%$. RDX also shows a very high MS/MS efficiency. The parent ions used for the nitroaromatics, which are more kinetically stable than those for RDX and PETN, tend to show efficiencies of 40-60\% [56]. These efficiencies are one to two orders of magnitude greater than those obtained with beam-type tandem mass spectrometers. 

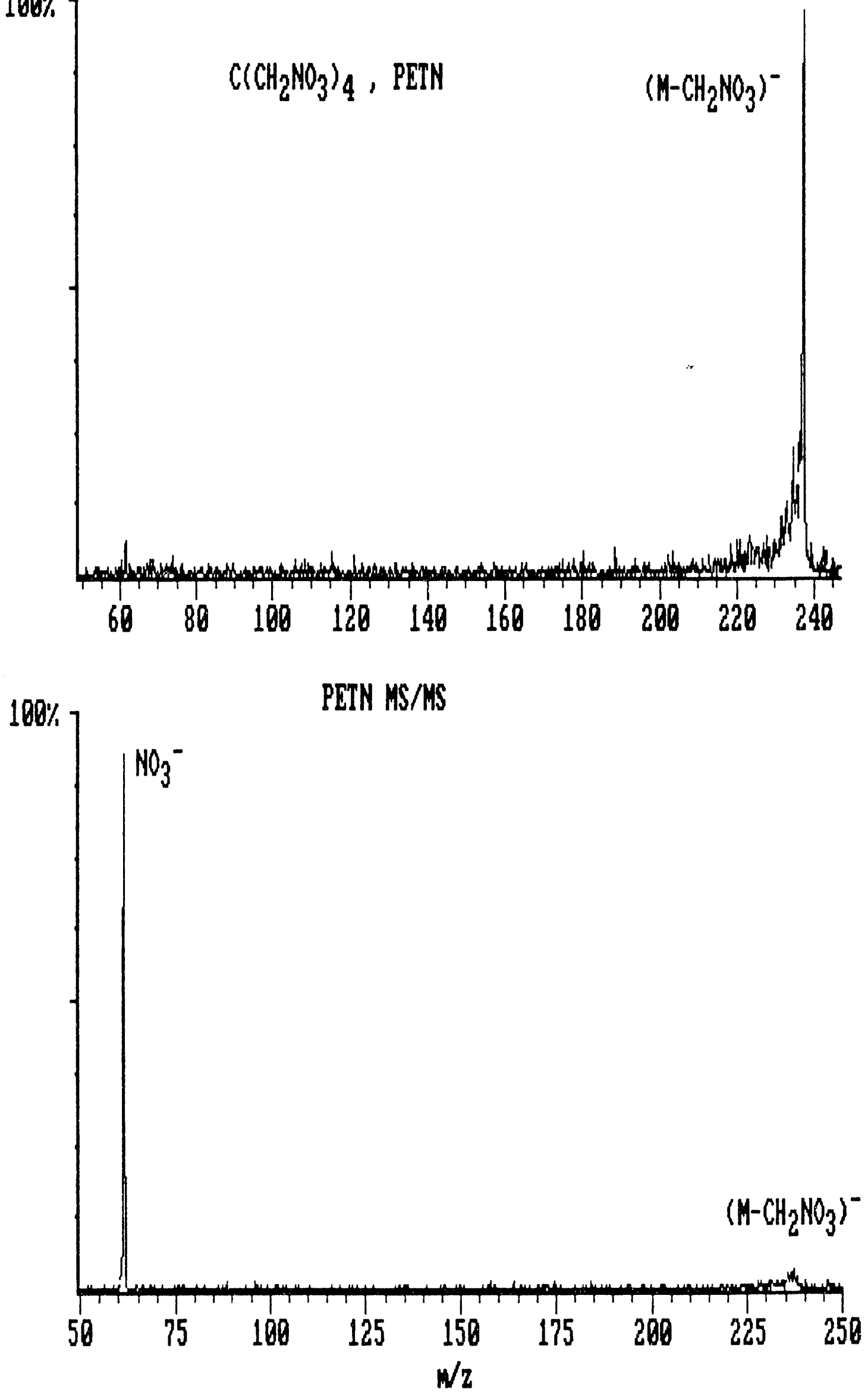

Figure 17. $\mathrm{MS} / \mathrm{MS}$ spectra of $\mathrm{m} / 7$ 240, $\left(\mathrm{M}-\mathrm{CH}_{2} \mathrm{ONO}_{2}\right)$, from PFTN (top) spectrum of isolated parent ion (bottom) spectrum after resonance excitation. 
The typical ion trap MS/MS experiment consists of a set of sequential steps beginning with ion formation and accumulation. After the ionization step is completed, further ion accumulation is prevented during the ion manipulation steps. The first ion manipulation step is mass selection effected by one of the methods described above. Collisional activation is then effected either using a single frequency applied to the end-caps or a range of frequencies. The latter approach is newer and overcomes problems associated with tuning and ion-ion interactions [57]. The final step involves mass analysis of the product ions using one of the several mass analysis approaches. A particular advantage of ion trapping instruments is that the mass selection/collisional activation sequence can be repeated using a product ion formed in a previous CID reaction as the parent ion for a subsequent CID experiment. This procedure is commonly referred to as an $M^{n}$ experiment where $n$ represents the number of stages of mass spectrometry. As many as 13 stages of MS have been demonstrated in a quadrupole ion trap [58]. The factors that determine the maximum value of $n$ have been discussed [59]. The capability for $M^{n}$ where $n>2$ is a distinct advantage for ion trapping instruments relative to beam-type instruments which require a separate mass analyzer for each stage of MS. The specificity of an analysis may be enhanced dramatically by additional stages of mass spectrometry provided unique information is obtained with each step. For the explosives detection application, we have yet to encounter a situation in which a third stage of MS is desirable but the ion trap can provide this capability if necessary with a very minor change in software.

Conventional ion trap mass spectrometry ionizes vapors present in the ion trapping volume by electron ionization using a filament mounted on an end-cap. This approach has been shown to be able to detect volatile organics in air down to the low parts per billion by volume level in the positive ion mode. However, electrons are not stored within the ion trap under conditions for efficient ion storage. Therefore, electron capture is a very inefficient process due to the fact that high number densities of low energy electrons cannot be maintained in the ion 
trap. For this reason, we interfaced an ASGDI source with an ion trap to perform the anion formation externally $[60,61]$. Ions are continuously formed in the ASGDI source and are gated into the ion trap during the ion accumulation period that begins every ion trap experiment. The "duty cycle" of the ion trap experiment employing a continuous ion source, defined as the fraction of time that ions formed by the ionization method can be measured, is the ion accumulation time divided by the entire time of the experiment. Any analyte ions formed during the ion manipulation and mass analysis steps are not permitted to accumulate in the ion trap and are therefore lost. The duty cycle in a typical experiment can range from a few percent to over $90 \%$. This is an advantage of the ion trapping instruments in that ion accumulation is variable and can be as long as necessary, within trapping efficiency limitations, to accumulate a sufficient number of ions for analysis. Therefore, long ion accumulation times can be used to integrate ions from a weak beam issuing from the ion source whereas short accumulation times are sufficient to accumulate ions from a strong beam. The duty cycle of a beamtype mass spectrometer, on the other hand, is determined by the fraction of time that the spectrometer scans over the $\mathrm{m} / \mathrm{z}$ range of the analyte ion divided by the total scan time. For a mass/charge range of 50-350, for example, the duty cycle for any particular ion is $1 / 250$ or $0.4 \%$. Duty cycle can be increased to $100 \%$ in the single ion moritoring mode, but thereby results in a duty cycle of $0 \%$ for any other ion of interest. There is, therefore, an important compromise between duty cycle and mass range in a beam-type instrument that is largely absent with the ion trap. The ion trap can accumulate ions over a wide mass range simultaneously while a wide mass range can be analyzed in a relatively short period of time (about 50 milliseconds whereas ion accumulation can range from 1-2000 milliseconds). The ion trap therefore enjoys something of a Felgett's advantage over the scanning beam-type instruments.

The parallels and perpendiculars of ion trap vs. beain-type instrumentation is a big subject, certainly too big for this report. However, the purpose here is to mention enough of the major points to appreciate the merits of these technologies 
for explosives detection. Therefore, the figures of merit discussed in the previous section for beam-type tandem mass spectrometers, with emphasis on the triple quadrupole instrument, are discussed here for the quadrupole ion trap operated mated with ASGDI and using the techniques described above. Important characteristics of the quadrupole ion trap relevant to this discussion include:

- Sensitivity - Sub-picogram detection limits for explosives can be achieved for explosives-related ions both in the MS and in the MS/MS modes under favorable conditions. The detection limits in the MS/MS mode are clearly superior with the ion trap relative to the beam-time instruments. As discussed below in the comments about dynamic range, the ion trap suffers from ion-ion interactions much more so than beam-type instruments. This problem has been overcome to a large extent with mass-selective ion accumulation methods. However, there remains the possibility for a very intense ion to appear at the same mass/charge as a targeted analyte ion that might affect the accumulation of the ion of interest. We have not encountered such a situation for the species of general interest to the explosives detection community but the potential exists. Fortunately, there are possible approaches to overcome even this problem if it arises. As mentioned above, the favorable duty cycle of the ion trap and the ability to accumulate ions over a wide range of masses simultaneously is an advantage for the ion trap. However, experiments with a reliable vapor generator with both types of technologies is desirable to determine if single ion monitoring with a beam instrument ( $100 \%$ duty cycle) is significantly superior to long ion accumulation time ion trap analysis.

- Specificity - The mass resolving power of the ion trap is typically on the order of 1000 in both stages. Mass resolution can be higher by slow scanning but at the expense of duty cycle. As mentioned above, $\mathrm{MS}^{\mathrm{n}}$ is readily performed in the ion trap as well. Therefore, 
the capability for high resolution and $\mathrm{MS}^{n}$ provided by the ion trap can give extremely high specificity, if needed. This level of potential specificity is not afforded with the triple quadrupole instrumentation. From the practical point of view, however, the specificities of the ion trap and triple quadrupole instruments as they are currently used for explosives detection are comparable and appear to be quite satisfactory for the application.

- $\quad$ Speed - The sequence of steps for an ion trap MS/MS experiment can range in duration from 50 milliseconds to over one second. This time range is determined primarily by the ion accumulation time. The analysis time difference between the ion trap and triple quadrupole instruments is not significant in the context of the entire time needed for sampling, preconcentration, and analysis.

- $\quad$ Size - The ion trap enjoys a distinct advantage in terms of size and weight. A single analyzer and set of electronics provides MS/MS capabilities and the ability to tolerate high background pressures (at least $10^{-4}$ Torr of air has been used routinely) minimizes pumping requirements. The engineering involved in designing a relatively small footprint system (e.g., the size of a PC, for example) should be straightforward.

- Cost - lon traps are the least expensive tandem mass spectrometers. Costs associated with electronics, pumping, and machining are lower than for the triple quadrupole mass spectrometer, for example. It is not difficult to imagine that an ion trap explosives detector can be marketed for less than $\$ 100 \mathrm{~K}$ given the fact that ion trap GC/MS systems are marketed for less than $\$ 50 \mathrm{~K}$.

- Dynamic range - As mentioned above, ion traps encounter ion-ion interaction problems much sooner than do beam-type instruments. These problems have been addressed to a large extent by massselective ion accumulation methods and by universal collisional 
activation methods. Nevertheless, dynamic range is expected to be superior with the beam-type instruments. Quantitative measurements of dynamic range with the newer techniques have not yet been made but preliminary measurements suggest ion trap dynamic ranges of $10^{5}$ can be achieved in mixture analysis applications.

- Scan Flexibility - Parent ion, neutral loss, and targeted product ion scans are much less conveniently performed with the ion trap than with the triple quadrupole instrument. However, these experiments can be performed if need be. The fact that all experiments are performed with one analyzer simply by changing voltages applied to the electrodes makes the ion trap extremely flexible in the number and types of experiments that might be performed. In principle, provided the hardware to generate the necessary signals is under computer control, simple changes in software can make a wide variety of experiments possible including $M^{n}{ }^{n}$ studies.

From the point of view of developing a fieldable explosives vapor detector based on tandem mass spectrometry, the advantages enjoyed by the ion trap in terms of MS/MS sensitivity (see Section VII), size, and weight would seem to make it the technology of choice. However, the beam-type instrument remains a powerful tool for explosives analysis but would appear to be most appropriate in a laboratory-based setting. 


\section{THE FAA GLOW DISCHARGE/TRIPLE QUADRUPOLE TANDEM MASS SPECTROMETER}

In the spring of 1989, FAA officials visited ORNL and expressed interest in the ASGDI/tandem mass spectrometry work in explosives vapor detection with the goal of evaluating system integration approaches. At that time, our laboratory had been working with home-built tandem quadrupole/time-of-flight technology and had been developing ion trap technology for explosives detection. Also at that time, important criteria for an explosives vapor detector were high reliability and ready access to equipment service. The ion trap technology was deemed too immature at that point, and the quadrupole/time-of-flight instrumentation was unique to ORNL. For these reasons, we proposed to interface ASGDI with a commercially available triple quadrupole system. First, beam-type technology was mature and well-understood and second, the vendor could provide service engineering support for the triple quadrupole system. The ASGDI source is both rugged and reliable and it was not anticipated that there would be any reliability problems with ionization. A "triple stage quadrupole", model number TSQ700 manufactured by Finnigan Corporation of San Jose, CA, was therefore procured and modified for operation with ASGDI. The standard TSQ700 instrument, modifications for ASGDI operation, and performance characteristics of the system are described in the following sections.

\section{A. Finnigan Triple Stage Quadrupole (TSQ700) mass spectrometer}

A block diagram of the mass analyzer assembly for the TSQ700 instrument, unmodified for use with the ASGDI source, is shown in Figure 18. The unit is comprised of an electron ionization (EI) / chemical ionization (CI) ion source, two quadrupole rod assemblies, one octapole rod assembly, ten ion lenses, and a detector assembly. Beginning from the ion source end of the instrument, the rod assemblies are designated as Q1, Q2, and Q3. As noted earlier, although instruments of this type are generally referred to as "triple quadrupcle mass 


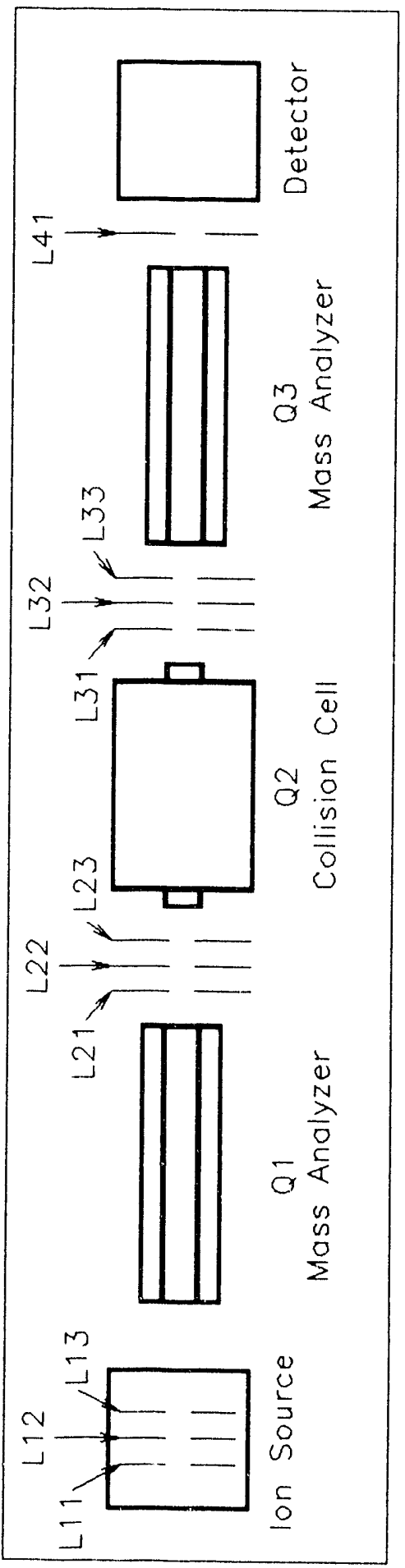

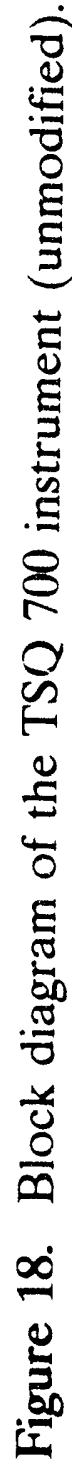


spectrometers", only Q1 and Q3 are configured to function either as mass analyzers or ion transmission devices. The Q2 assembly, which serves as the collision region when a collision gas is introduced, is operated only in the ion transmission (non-analyzing) mode thereby allowing ions over a broad mass/charge range to pass.

\section{1. $\mathrm{El} / \mathrm{Cl}$ ion source}

Although the ASGDI source is used in the TSQ700 as modified for explosives detection applications, the standard El / Cl ion source will be described briefly for completeness. The $\mathrm{El} / \mathrm{Cl}$ source produces an energetic election beam, provides an interaction region for electrons and sample molecules to generate ions, and focuses the ions into the mass analyzer assembly. Electrons are produced via thermionic emission from a heated rhenium filament. In the EI operational mode, the filament is normally maintained at $-70 \mathrm{~V}$ with respect to the interaction volume (ground) resulting in a $70 \mathrm{eV}$ electron beam for positive ion production. Ionization of sample molecules is a two-step process in the $\mathrm{Cl}$ operational mode. A reagent gas, introduced along with the sample vapors, is initially ionized via El. These reagent ions then form a variety of other stable ions via reaction with other reagent gas molecules. Sample ions of either polarity are then formed in the second step of the process; the sample ions can be generated by reactions such as proton transfer, proton abstraction, charge transfer, ionmolccule association, or electron capture of thermal electrons which are a byproduct of reagent gas ionization. During $\mathrm{Cl}$, the reagent gas is normally maintained at a pressure ( 0.5 Torr) several orders of magnitude greater than that for the sample. Thus, the electron entrance and ion exit holes for the $\mathrm{Cl}$ interaction volume are smaller than for the El interaction volume. A three-element ion source lens assembly, located just outside the interaction volume, aids transfer of sample ions to the mass analyzer assembly. The first element of the lens assembly (L11) extracts ions from the interaction volume. In positive ion mode, L11 is typically held at $-10 \mathrm{~V}$; the polarity is reversed in negative ion mode. The complete lens 
assembly $(L 11, L 12, L 13)$ functions as an aperture lens that focuses and injects the extracted ions into the mass analyzer. The central element, L12, is set to approximately $-50 \mathrm{~V}$ in positive ion mode and $50 \mathrm{~V}$ in negative ion mode. L13 is normally held at the same potential as L11.

\section{Q1 and Q3 mass analyzers}

Each of the two quadrupole mass analyzer assemblies (Q1, Q3) consists of a square array of four hyperbolic rods. The individual rods are $16 \mathrm{~cm}$ long, and the spacing between opposing rods is $8 \mathrm{~mm}$. In each array, rods opposite each other are connected electrically with the voltages applied to each rod pair equal in amplitude but opposite in polarity. At any given instant, if and dc voltages in a variable ratio are applied to the rods, the possible (constant frequency, 1 $\mathrm{MHz}$ ) if potential varying between 0 and $6000 \mathrm{~V}(\mathrm{p}-\mathrm{p})$ and the dc potential from 0 to $\pm 500 \mathrm{~V}$. During ion transit along the long axis of the quadrupole assembly, the electrodynamic fields produced by the applied voltages induce oscillatory ion motion normal to that axis. The range of mass/charge values which maintain stable trajectories through the entire length of the quadrupole array is determined by the $\mathrm{rf} / \mathrm{dc}$ ratio. A mass spectrum can be acquired by increasing the $\mathrm{rf}$ and $\mathrm{dc}$ voltages while maintaining a fixed $\mathrm{rf} / \mathrm{dc}$ ratio so that ioris are transmitted sequentially from low to high mass/charge. The potentials on each array can be varied rapidly and independently, so that the full mass range $(10-2000 \mathrm{u})$ of Q1 or Q3 can be scanned in as little as $200 \mathrm{~ms}$.

\section{Q2 assembly}

The Q2 assembly is a non-linear array of eight cylindrical rods. Alternate rods are connected electrically with the if (constant frequency, $2 \mathrm{MHz}$ ) voltage applied to one set of four rods equal in amplitude but opposite in polarity to the other set. The resultant electrodynamic field allows ions over a wide range of mass/charge values to be passed. The curvature of the octapole rods in the direction of ion transmission has negligible effect on ion transmission, the typical 
efficiency being $90-100 \%$. However, because neutral particles and photons do not follow a curved trajectory, the net effect is to lower significantly the noise originating from those sources.

Q2 is housed in a shroud enabling the assembly to serve as a collision cell when pressurized ( 0.1 - 10 mTorr) with a buffer gas. Ion losses in Q2 are reduced by the presence of the $\mathrm{if}$ field so that product ions produced via CID are efficiently collected and transmitted to mass analyzer Q3. The collision energy in MS/MS experiments is determined by the magnitude of the potential difference between the ion source (ground) and the dc offset voltage applied to Q2. The collision energy, which is normally fixed during any MS/MS scan, can be varied from 0 to $\pm 200 \mathrm{~V}$.

4. Analyzer assembly lenses

The analyzer assembly of the TSQ700 contains seven ion lenses which are arranged in three sets. Lenses L21, L22, and L23 are positioned between Q1 and Q2; L31, L32, and L33 are located between Q2 and Q3; and L41 is situated between Q3 and the detector. Each lens has a circular aperture at its center through which the ion beam passes. The L2x (and L3X) lenses together form $a$ three-element lens as in the ion source. Just as in the ion source, the first and third elements are normally held at the same voltage with the central lens set to some different voltage. The voltages applied to any of the seven ion lenses can be varied from -200 to $200 \mathrm{~V}$.

One of the functions of the $L 2 x$ and $L 3 x$ lens sets is to focus the ion beam emerging from the preceding rod assembly into the following. Another function of L2x and L3x is to shield adjacent rod assemblies from the voltages applied to each. The most important function of $L 2 x$ and $L 3 x$ is to minimize the buffer gas flow from Q2 into the mass analyzers Q1 and Q3. To aid in this regard, L23 and L31 form the two ends of the collision cell Q2 and L21, L22, L32, and L33 act as additional baffles to help prevent buffer gas that escapes the collision cell from entering the mass analyzers. 
5. Lotector

The TSQ700 is equipped with a continuous dynode electron multiplier ion detection system having an off-axis conversion dynode that reduces noise from neutral particles and photons. The conversion dynode, located at a right angle to the entering ion beam, is a concave metal surface. The voltage applied to the coniversion dynode is typically in the range \pm 5 to $\pm 15 \mathrm{kV}$. Raising the dynode voltage (magnitude) tends to improve ion detection by increasing the primary ion to secondary particle conversion efficiency, enhancing the number of seconda $y$ particles generated per conversion, and increasing both factors for ions of relatively high mass/charge. The time required to switch the dynode polarity is aprroximately equal to the interscan time $(80 \mathrm{~ms})$, thus the detector can rapidly be sintiched between positive-ion and negative-ion detection modes. The cor.inuous dynode electron multiplier is also positioned normal to the entering ion beam ari $4 r$ "rectly or posite the conversion dynode. The entrance end of the cathode is normally operated between -1 to $-3 \mathrm{kV}$, and the exit end of the cathode (and the anode) is at ground.

\section{Vacuum system}

The TSQ700 vacuum manifold, which houses the ion source, analyzer assembly, and detector, divided into two regions by a baffle allowing efficient differential pumping: the front region contains the ion scurce, and the rear region contains the analyzer assembly and the dtiector. The kraffle is sealed to ion source lens L13, so that the small aperture in that lens is the only means by which gas can flow between the two regions of the vacuum manifold. Each of the regions is evacuated by a 300 liter- $\mathrm{S}^{-1}\left(\mathrm{~N}_{2}\right)$ turbomolecular vacuum pump that in turn is backed by a 300 liter-min ${ }^{-1}\left(N_{2}\right)$ rotary-vane pump. Gas inlet lines are evacuated by a separate 165 liter- $\min ^{-1}$ rotary-vane purnp. Depending on the introduction rate of gases into the ion source and collision cell, the operating pressure of the arialyzer region is $10^{5}$ to $10^{7}$ Torr. 


\section{B. Scan capabilities}

As previously noted, quadrupole assemblies Q1 and Q3 can be operated independently in ion transmission ( $\mathrm{f}$ only) or mass analysis ( $\mathrm{ff}$ and dc) modes; Q2 operates with if voltage only and thus always functions as an ion transmission device. Given the flexibility of applying voltages to the quadrupole assemblies, the TSQ700 can be operated in a variety of scan modes. For situations in which Q1 or Q3 is used as an ion transmission device, the other is used in mass analysis mode, and Q2 is operated in ion transmission mode, the scan mode is single MS. When Q1 or Q3 is operated in ion transmission mode and the other functions as a mass analyzer or when both operate in mass analysis mode, and Q2 also functions as a collision cell, the scan mode is designated as MS/MS. Table III summarizes the scan modes and the functions of the rod assemblies for each. A brief description of each scan mode is also presented below.

\begin{tabular}{|c|c|c|c|}
\hline Scan Mode & Q1 & Q2 & Q3 \\
\hline Q1MS & Scan & Pass all ions & Pass all ions \\
\hline Q3MS & Pass all ions & Pass all ions & Scan \\
\hline Parent MS/MS & Scan parent ions & $\mathrm{CID}$ & Select product ion \\
\hline Product MS/MS & Select parent ion & CID & $\begin{array}{c}\text { Scan product } \\
\text { ions }\end{array}$ \\
\hline Neutral Loss MS/MS & Scan parent ions & CID & $\begin{array}{c}\text { Scan product } \\
\text { ions }\end{array}$ \\
\hline $\begin{array}{c}\text { Targeted Product } \\
\text { MS/MS }\end{array}$ & Pass all ions & CID & $\begin{array}{l}\text { Select product } \\
\text { ion(s) }\end{array}$ \\
\hline
\end{tabular}

1. Q1MS and Q3MS

In Q1MS and Q3MS modes, only a single stage of mass analysis is performed by Q1 or Q3, respectively, on ions formed in the ion source. Q2 acts as an ion transmission device. 


\section{Parent MS/MS}

Two stages of mass analysis are executed in the parent ion MS/MS mode of operation. First, ions generated in the ion source are transmitted to the collision cell (Q2) following sequential mass analysis in Q1. Following CID (or metastable decomposition) of parent ions in Q2, the product ions enter Q3 which is set to pass a specific produst ion. Thus, the spectrum produced in a parent ion scan shows all parent ions which dissociate to give a specific product ion. The abscissa in the parent MS/MS spectrum represents the mass/charge values passed by $\mathrm{Q} 1$, and the ordinate indicates intensity values for the product ion monitored by Q3.

\section{Product MS/MS}

The roles of $\mathrm{Q} 1$ and $\mathrm{Q} 3$ in product MS/MS mode are reversed from those performed in parent MS/MS mode. Q1 selects a specific mass/charge ratio for ions produced in the ion source and transmits those parent ions to the collision cell. As in parent MS/MS, product ions can be produced in Q2 via CID or metastable decomposition. Product ions formed in the collision cell then enter Q3 where they are mass analyzed to obtain a spectrum which shows all product ions formed by fragmentation of the selected parent ion.

\section{Neutral Loss MS/MS}

In the neutral loss MS/MS scan, Q1 and Q3 are both operated in mass analysis mode. They are scanned simultaneously with a fixed difference between mass/charge values transmitted; the mass/charge value passed by $\mathrm{Q} 1$ is always greater than that passed by Q3. Loss of a neutral species occurs in the collision cell via CID or metastable decomposition. Therefore, a neutral loss MS/MS spectrum represents all parent ions formed in the ion source that lose a neutral moiety of specified mass. As in the parent MS/MS spectrum, the transmitted parent ion mass/charge is shown on the abscissa and intensity data for the product ion monitored by Q3 are indicated on the ordinate. 


\section{Targeted Product MS/MS}

In targeted product MS/MS mode, Q1 is operated in the ion transmission mode enabling virtually all ions formed in the ion source to enter the collision cell. As in the other MS/MS scan modes, fragment ions produced in Q2 via CID and metastable decomposition are then transmitted to Q3. Only a single stage of mass analysis occurs in targeted product MS/MS mode, that being performed by Q3 to select product ions of a particular mass/charge. Thus, the targeted product MS/MS spectrum indicates the presence of any parent ions which fragment to give a specific product ion.

\section{ASGDI-TSQ700 interface}

In order to adapt the ASGDI to the TSQ700, the front flange, filament assembly, and electron interaction region were removed from the standard $\mathrm{El} / \mathrm{Cl}$ ion source assembly. They were replaced with a custom-designed, one-piece front flange/ASGDI source assembly as diagrammed in Figure 19 and pictured in Figure 20. More detailed schematic drawings of the new ion source assembly are shown in Appendix 1. The front and rear aperture plates, which have aperture diameters of 175 and $250 \mu \mathrm{m}$, respectively, are separated by $2.5 \mathrm{~cm}$. A $10 \mathrm{~L}_{-} \mathrm{s}^{-1}$ rotary vane pump evacuates the discharge region via three $5 / 8 \mathrm{in}$. diameter channels machined in the source block. This pumping speed coupled with the air sampling aperture in the front aperture plate results in an inlet flow rate of 2.5 $\mathrm{mL} / \mathrm{s}$. A thermal conductivity gauge inserted in a fourth channel enables the source pressure ( 0.8 Torr) to be monitored via a thermal conductivity gauge. The glow discharge is powered by an additional dc power supply ( $\pm 500 \mathrm{~V}, 10 \mathrm{~mA}$ ) connected to the front aperture plate; the rear aperture plate is grounded. The new ion source extends into the vacuum manifold so that its exit aperture plate is $1.5 \mathrm{~cm}$ from $\mathrm{L} 11$ of the $\mathrm{L} 1 \mathrm{X}$ three-element $\mathrm{El} / \mathrm{Cl}$ lens assembly. $\mathrm{L} 11$ was replaced with a tubular $(1 \mathrm{~cm}$ ) extraction lens (designated L1T in Figure 19). L1T is powered by the same voltage supply previously used to control L11. L1x lens elements 112 and L13, which also functions as a baffle between the ion source 


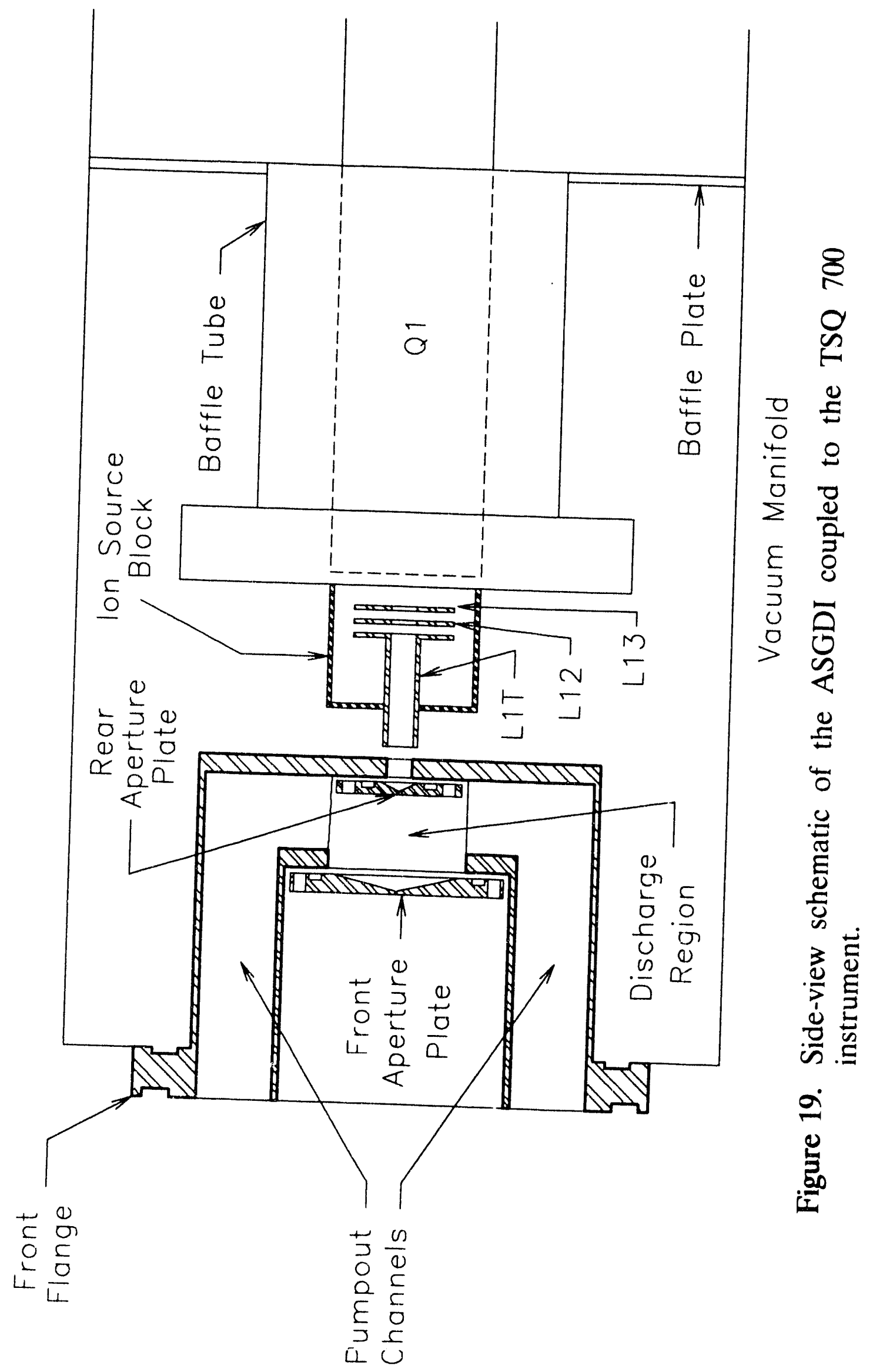



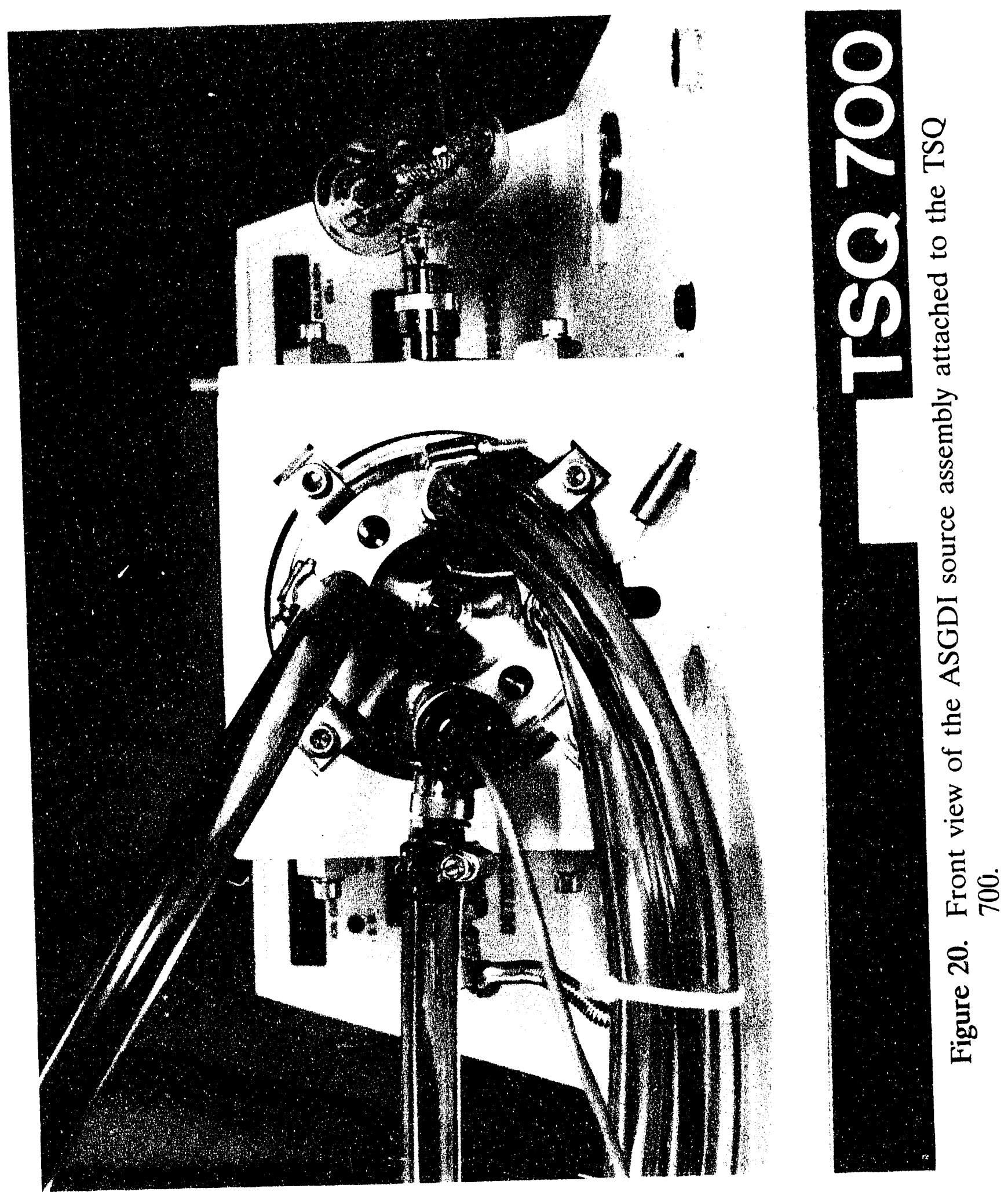
and analyzer regions of the vacuum manifold, were retained. Figure 21 is an overall view of the TSQ700 instrument with the ASGDI source assembly installed. This photo does not show the associated work station and the gas chromatograph that was also delivered with the TSQ700 system. 


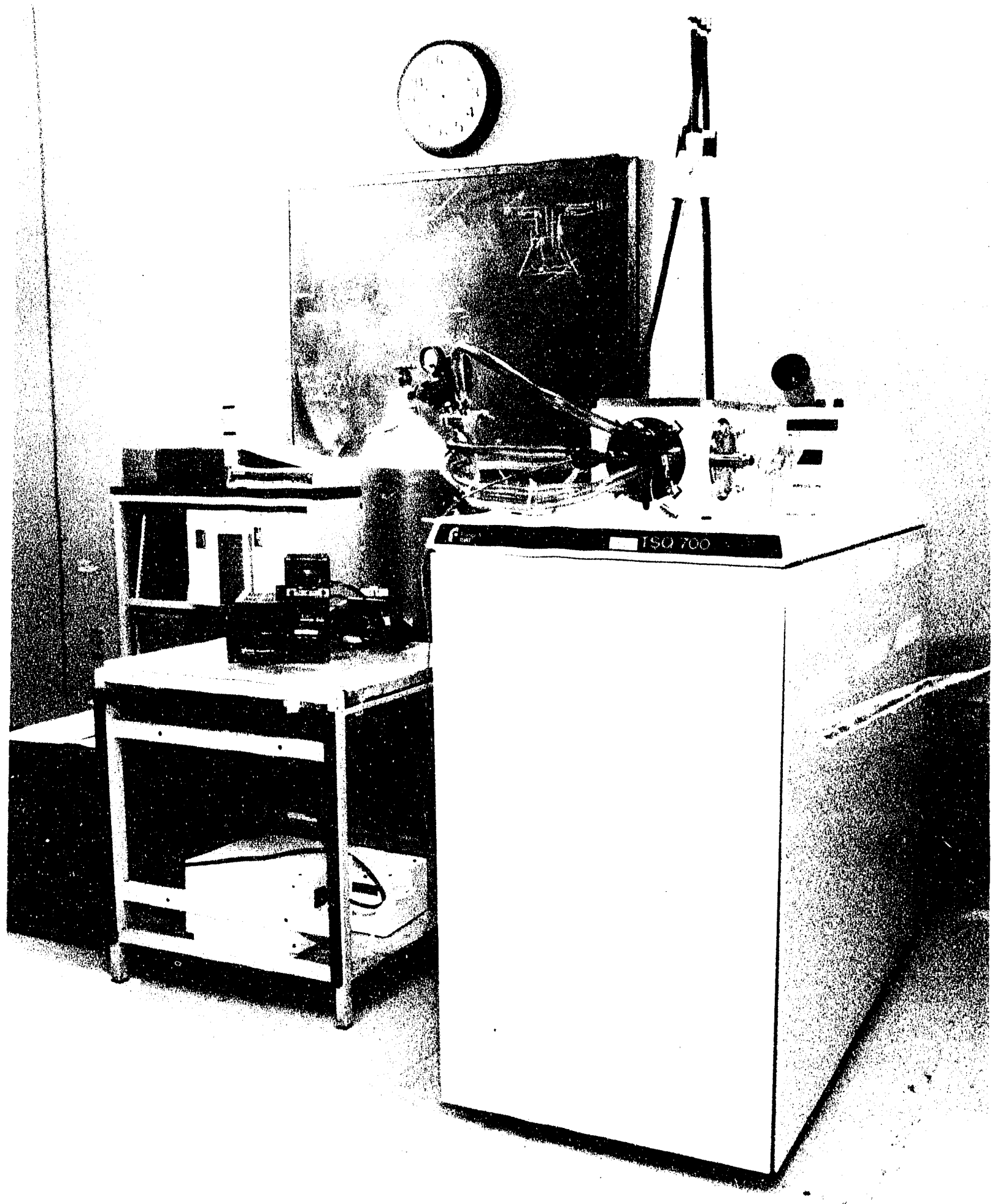

Figure 21. Full view of the TSQ 700 with the ASGDI source assembly installed. 


\section{PERFORMANCE CHARACTERISTICS OF THE FAA ASGDI/TSQ 700}

The performance of the ASGDI source on the TSQ 700 was characterized by a number of experiments using the anions derived primarily from several explosives; 2,4,6-trinitrotoluene (TNT), a nitroaromatic; 1,3,5-trinitroazocyclohexane (RDX), a heterocyclic nitramine; and pentaerythritol tetranitrate (PETN), a nitrate ester. All data were acquired in the negative ion mode by placing 350-400 V on the cathode (front aperture plate) which results in a discharge current of 4-8 mA. The mass spectra were acquired by scanning the second mass analysis quadrupole, Q3. Various approaches for acquiring MS/MS data were utilized. Samples were introduced into the ASGDI source either as room temperature head space vapors or by placing a few crystals in a capillary tube and placing the capillary in a heated quartz tube leading to the inlet aperture of the ASGDI source.

\section{A. Mass Spectrometry}

The mass spectra obtained on the ASGDI/TSQ 700 for 2,4,6,-trinitrotoluene (TNT), pentaerythritol tetranitrate (PETN), and cyclo-1,3,5-trimethylene-2,4,6trinitramine, commonly referred to as RDX, are shown in Figure 22. The background mass spectrum obtained from normal laboratory air in the absence of explosives is shown in Figure 23. These mass spectra are virtually identical to those obtained on other instruments using the ASGDI source at similar pumping speeds and at similar pressures. Note that the background mass spectrum is dominated by the peak at $\mathrm{m} / \mathrm{z} 46$, which is attributed to $\mathrm{NO}_{2}$. This species is formed predominantly in the ASGDI source by discharge in air, and does not indicate large concentrations of neutral $\mathrm{NO}_{2}$ in the atmosphere. A variety of other peaks is also consistently observed under these conditions. For example, signals appear at $\mathrm{m} / \mathrm{z} 16$ and 17 and are attributed to $\mathrm{O}$ and $\mathrm{OH}$, respectively. The molecular anion of oxygen, $\mathrm{O}_{2}^{-}$is also consistently observed. The small signals at $\mathrm{m} / \mathrm{z} 35$ and 37 are due to the isotopes of chlorine, $\mathrm{Cl}^{-}$. These ions do not appear consistently in the mass spectrum but do so when there is a freon leak in 
MS of TNT

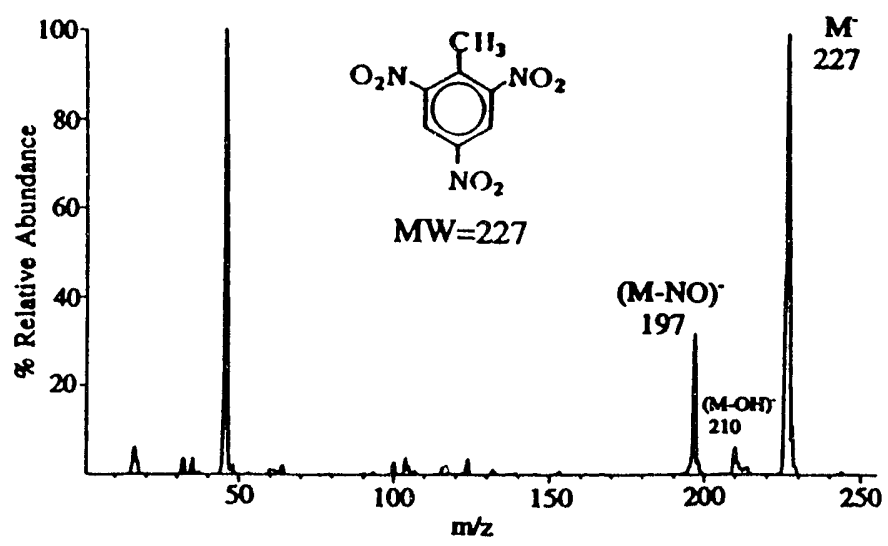

MS of PETN

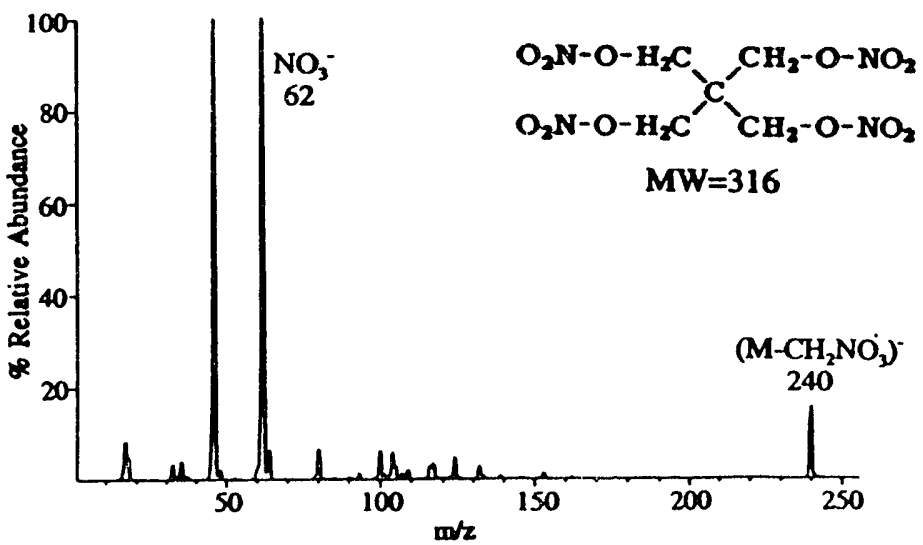

MS of RDX

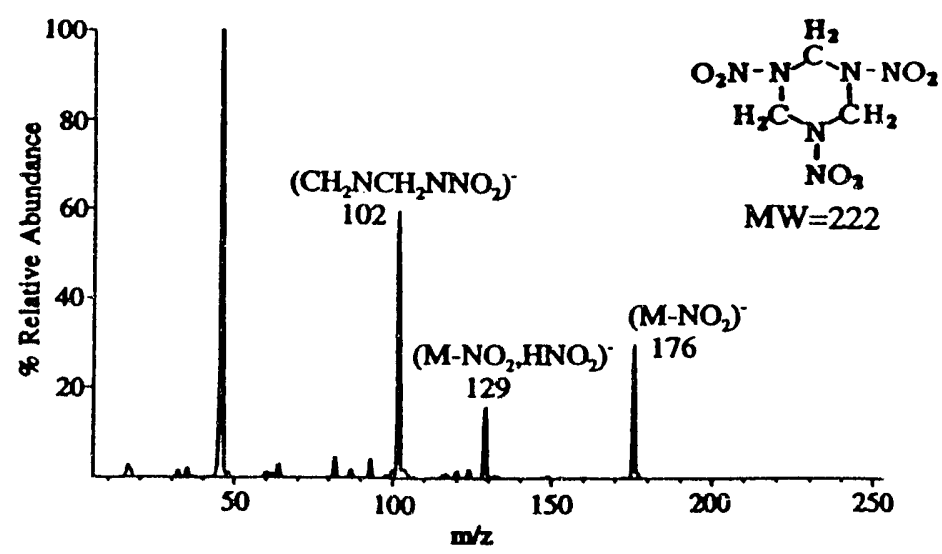

Figure 22. Mass spectra obtained on the ASGDI/TSO700 for TNT, PETN, and RDX. 


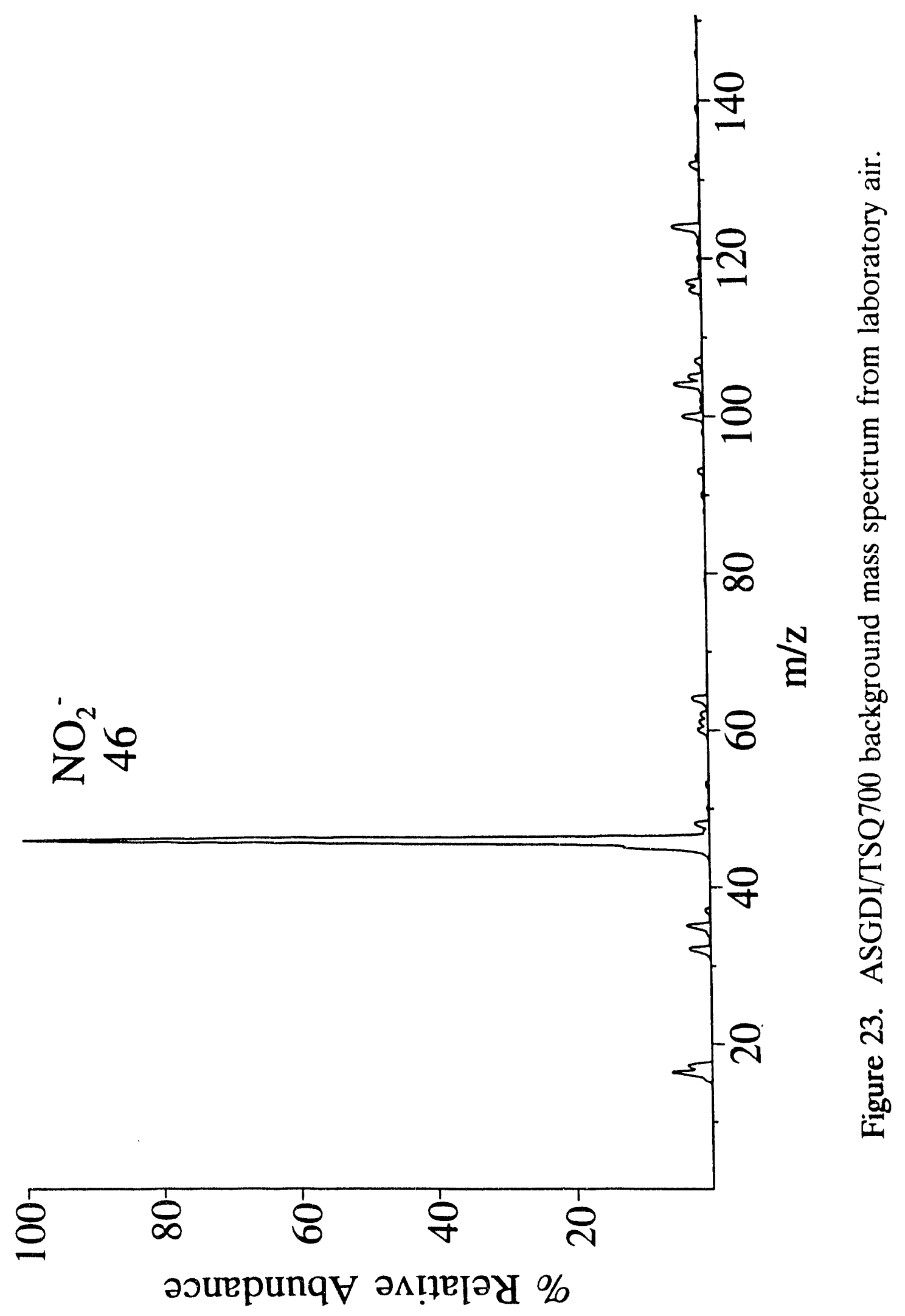


the building or when some chlorine-containing solvent is in use, as must have been the case when the spectrum of Figure 23 was acquired. An ion arising from ozone at $\mathrm{m} / \mathrm{z} 48, \mathrm{O}_{3}$, is almost always observed. Ozone is expected to be formed in an air-sustained electrical discharge. Mono- and di-hydrates of $\mathrm{O}_{2}$, viz. $\mathrm{O}_{2}\left(\mathrm{H}_{2} \mathrm{O}\right)$ and $\mathrm{O}_{2}\left(\mathrm{H}_{2} \mathrm{O}\right)$, are often observed and appear at $\mathrm{m} / \mathrm{z} 50$ and $\mathrm{m} / \mathrm{z} 68$, respectively. The appearance of these ions is highly dependent upon the temperature and humidity of the air drawn into the ASGDI and upon the degree of moisture present on the cathode. Peaks at $\mathrm{m} / \mathrm{z} 60,61$, and 62 are always observed and correspond to $\mathrm{CO}_{3}, \mathrm{HCO}_{3}$, and $\mathrm{NO}_{3}$, respectively. All such species are presumably formed via ion/molecule reactions initiated by discharge in air. There are typically a variety of peaks from m/z 100 to $\mathrm{m} / \mathrm{z} 140$ that appear consistently. The identities of all of the species that give rise to these peaks have not been firmly established. However, pulsed discharge experiments with time-resolved mass spectrometry have demonstrated that the ions that give rise to these peaks have signilicantly longer formation times than the lower mass species just identified. The different time-dependent behavior of the background anions is interpreted as a reflection of differences in the ion formation mechanisms. Fast mechanisms are those that rely on electron capture and ion/molecule reactions involving high concentrations of reactants, such as oxygen and nitrogen. The low mass anions are probably formed by fast mechanisms. The higher mass anions are probably formed from multi-step reaction sequences which may well involve highly reactive neutrals generated within the discharge. While the identities of these species have not been clearly established, MS/MS results indicate that they are oxides of nitrogen. For example, the anion at $\mathrm{m} / \mathrm{z} 100$ is tentatively proposed to be $\mathrm{N}_{6} \mathrm{O}$.

All of the mass spectra of Figure 22 show a prominent signal at $\mathrm{m} / \mathrm{z} 46$ which arises primarily from the background $\mathrm{NO}_{2}$ formed in the source, although the formation of $\mathrm{NO}_{2}$ from some analyte species cannot be precluded. The nitroaromatics, for example, show formation of $\mathrm{NO}_{2}^{-}$in the $\mathrm{MS} / \mathrm{MS}$ spectra (see below). TNT, which is illustrative for nitroaromatics, gives primarily the molecular 
anion under the conditions used here (see also the results for 2,4-dinitrotoluene discussed in Section III). Smaller signals are also observed that arise from the loss of $\mathrm{OH}(\mathrm{m} / \mathrm{z} 210)$ and $\mathrm{NO}(\mathrm{m} / \mathrm{z} 197)$. The loss of a hydroxyl radical from nitroaromatic ioris is commonly observed when a hydrogen-containing substituent is ortho to a nitro group. This is a so-called "ortho effect" [63] and it has been studied in depth for explosives-related nitroaromatic anions [64]. The loss of NO is observed regardless of any other substituents on the ring and proceeds with an unusually large release of kinetic energy. This reaction has also been studied in some detail from the mechanistic standpoint [65]. The relative abundances of the fragment ions depend upon discharge voltage and pressure. The fragment ion peaks that appear in Figure 22 are relatively large. It is quite common to observe essentially no fragmentation in the mass spectrum for nitroaromatics.

PETN has a molecular weight of 316. Essentially no molecular anion of PETN is observed in the mass spectrum under normal conditions. We have observed molecular anions of PETN under conditions of high PETN concentration. The mechanisms for its formation under these conditions is unclear in light of the apparent lack of radical anion formation under low PETN concentration conditions. Perhaps a cluster ion is formed first under high concentration conditions and subsequently fragments to the molecular anion. Such a mechanism might account for the ability to observe intact molecular anions of PETN. Under low concentration electron capture conditions, which are most likely to prevail in an explosives detection scenario, the most intense high mass ion formed from PETN is observed at $\mathrm{m} / \mathrm{z} 240$ and corresponds to the molecular anion less a $\mathrm{CH}_{2} \mathrm{ONO}_{2}$ group. The major ion observed in the mass spectrum of PETN appears at $\mathrm{m} / \mathrm{z} 62$ and corresponds to $\mathrm{NO}_{3}$. We have already demonstrated that the nitrate ion formed from nitrate esters is the expected nitrogen-centered species whereas a significant fraction of the $\mathrm{NO}_{3}$ formed in the ASGDI source is the more weaklybound peroxy structure [25]. In any case, a high mass ion is preferred for MS/MS. Therefore, the $\left(\mathrm{M}-\mathrm{CH}_{2} \mathrm{ONO}_{2}\right)^{-}$ion is typically chosen as the parent ion for explosives detection. 
Like PETN, an intense molecular anion cannot be observed consistently for RDX. Several fragment ions are typically observed in the mass spectrum of RDX corresponding to the molecular anion less a nitro group $(\mathrm{m} / \mathrm{z} 176)$, the ion corresponding to $\left(\mathrm{M}_{-} \mathrm{NO}_{2}, \mathrm{HNO}_{2}\right)(\mathrm{m} / \mathrm{z}$ 129), and an ion corresponding to $\mathrm{CH}_{2} \mathrm{NCH}_{2} \mathrm{NNO}_{2}$ (m/z 102). As a rule, the highest mass species can provide the most structural information and is less likely to fall at the same $\mathrm{m} / \mathrm{z}$ as some uninteresting species. Therefore, the ion at $\mathrm{m} / \mathrm{z} 176$ is typically chosen as the parent ion for MS/MS. Although not shown here, HMX, the eight-membered ring analog to RDX (m.w. $=296)$ also shows an intense $\left(M-N_{2}\right)$ and behaves similarly under MS/MS conditions. These ions, as well as the $\mathrm{m} / \mathrm{z} 240$ ion from PETN, are quite satisfactory parent ions for MS/MS explosives detection.

\section{B. Mass Spectrometry/Mass Spectrometry}

There are several methods for acquiring MS/MS data on the TSQ 700. The coriventional method involves selecting the parent ion with the first mass analyzer (Q1), fragmenting the parent ion via collisional activation with a neutral target gas such as argon in the octapole region (Q2), and mass analyzing with the second mass analyzer (Q3). The resulting MS/MS spectra for TNT, PETN, and RDX are shown in Figures 24, 25, and 26, respectively. In the TNT MS/MS spectrum, fragments due to loss of $\mathrm{OH}(\mathrm{m} / \mathrm{z} 210)$ and $\mathrm{NO}(\mathrm{m} / \mathrm{z} 197)$ are the most dominant ions observed along with the $\mathrm{NO}_{2}$ ion $(\mathrm{m} / \mathrm{z} 46)$. The fragments arising from loss of $\mathrm{OH}$ and loss of $\mathrm{NO}$ were discussed above. The fact that they appear as the major fragment ions in the MS/MS spectrum confirms that they arise from the molecular anion. They are therefore formed in a step-wise fashion involving electron capture as the first step.

The only product ion observed in the MS/MS spectrum of the $\left(\mathrm{M}-\mathrm{CH}_{2} \mathrm{ONO}_{2}\right)$ (Figure 25) ion from PETN is $\mathrm{NO}_{3}$. As a rule, such a simple MS/MS spectrum is a mixed blessing in targeted compound analysis. On one hand, all of the product ion signal concentrated in a single peak is an advantage from the point of view of sensitivity. On the other hand, specificity may suffer since the identification is 


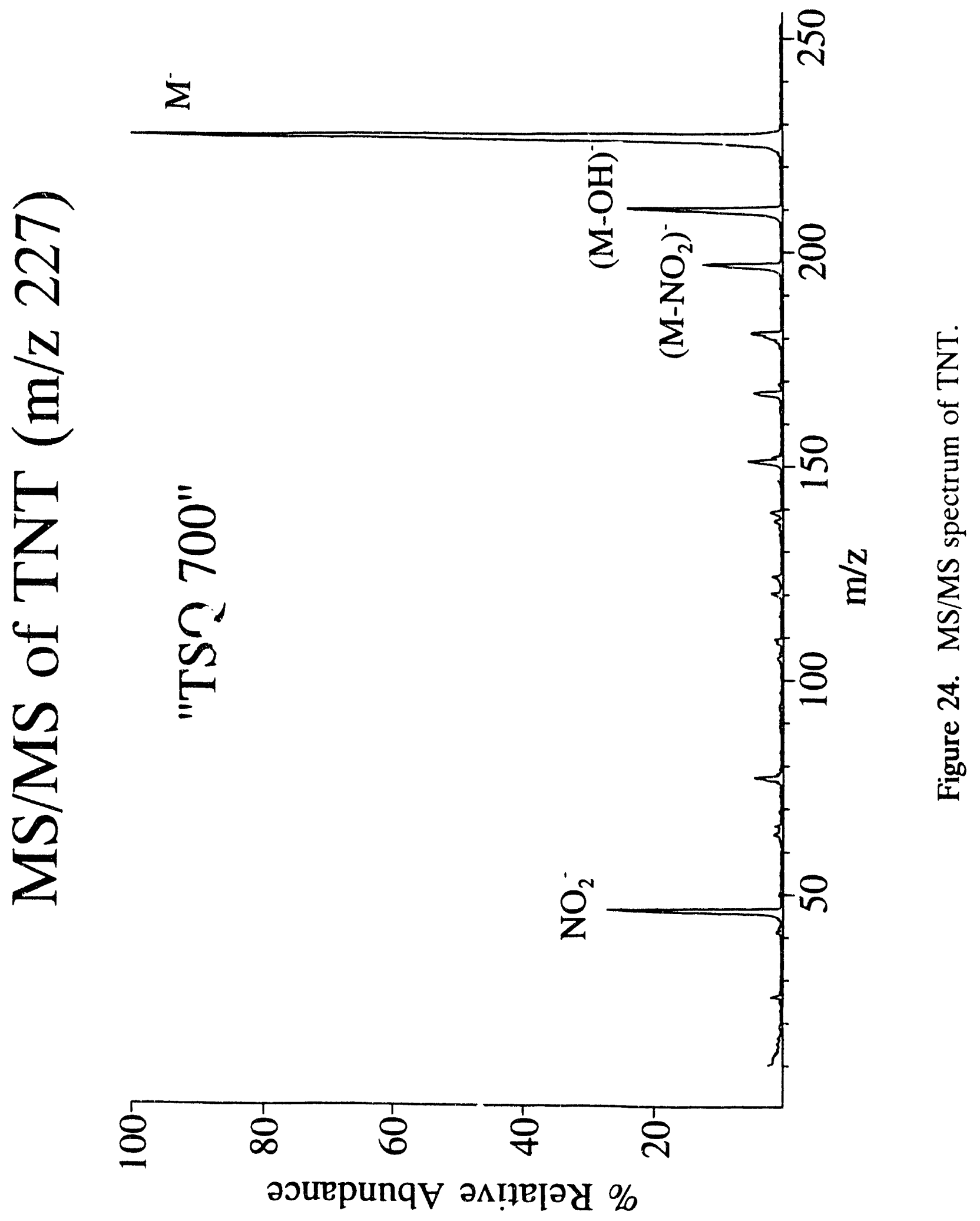




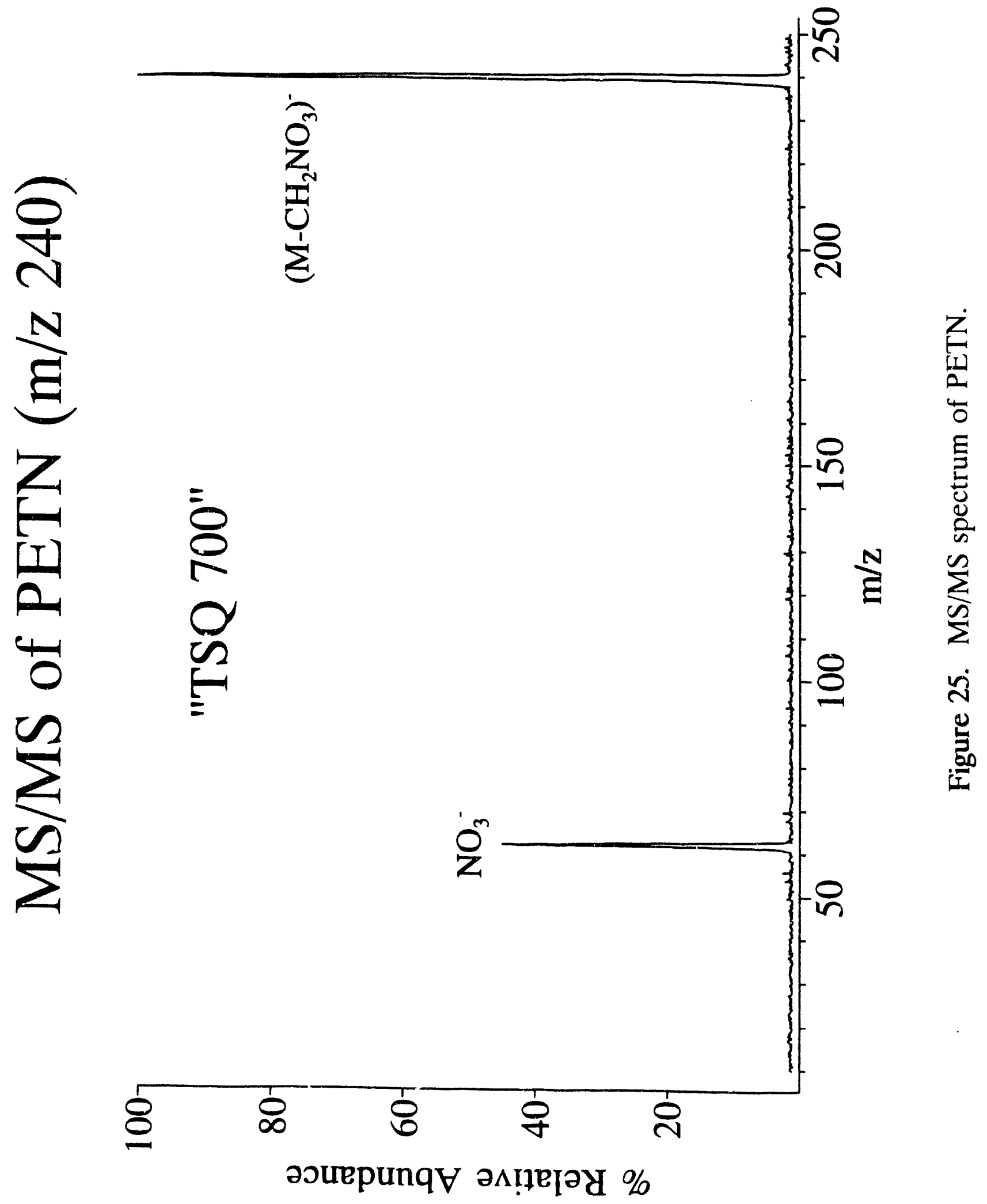




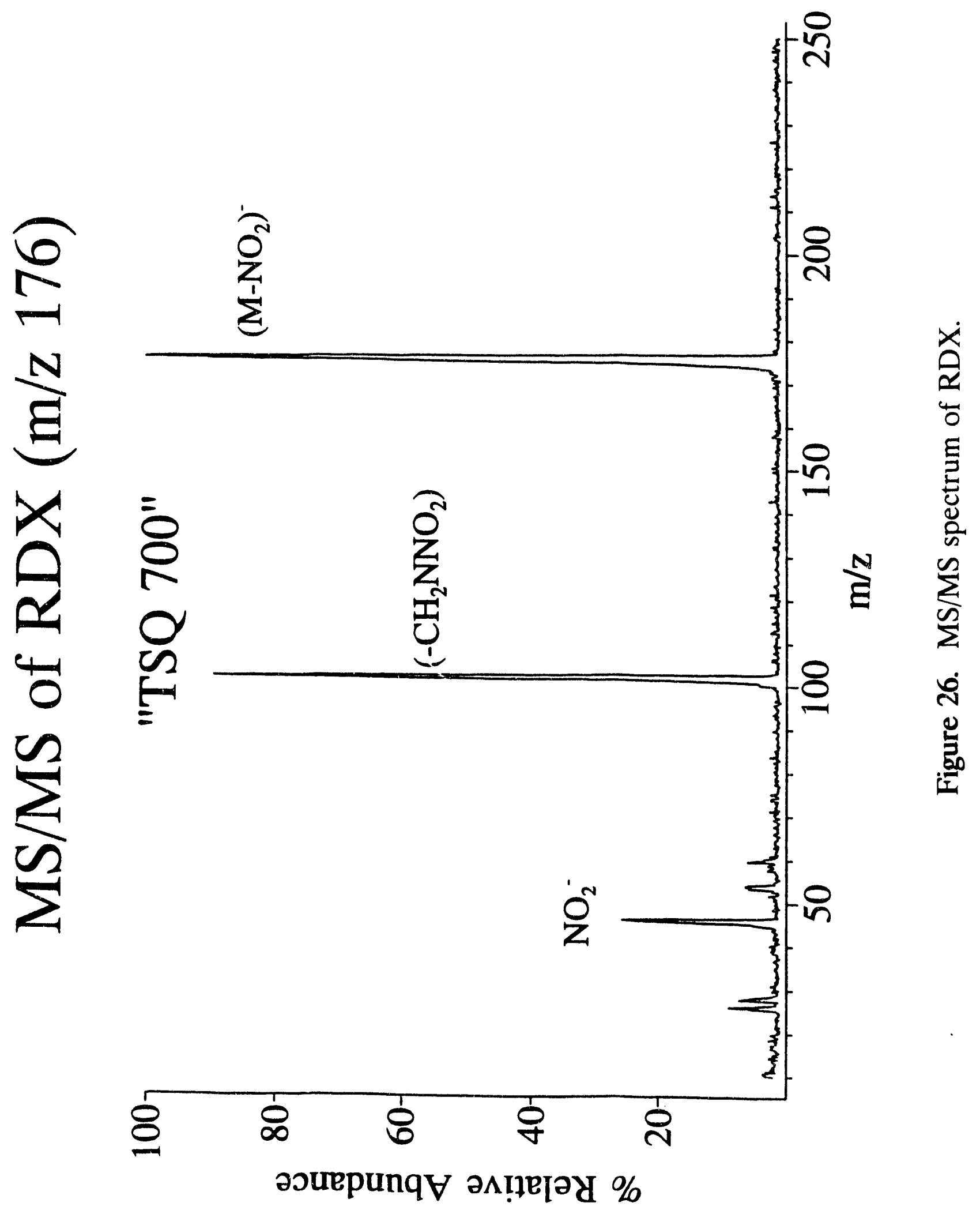


based on a single product ion. In this case, the 240-62 transition appears to provide quite adequate specificity as we have yet to observe any other compound behave in this manner.

The MS/MS spectrum of the $\left(\mathrm{M}-\mathrm{NO}_{2}\right)^{-}$ion from RDX $(\mathrm{m} / \mathrm{z} 176)$ yields a product ion from the loss of a $\mathrm{CH}_{2} \mathrm{NNO}_{2}$ group $(\mathrm{m} / \mathrm{z}$ 102) and the characteristic $\mathrm{NO}_{2}$ ion ( $\mathrm{m} / \mathrm{z}$ 46) (see Figure 26). The loss of $\mathrm{CH}_{2} \mathrm{NNO}_{2}$ from heterocyclic nitramine anions appears to be quite characteristic. Anions derived from HMX also behave in this way. Like the 240-62 transition with PETN, the 176-102 transition appears to be highly specific for PETN. If higher specificity is required, the coincident detection of the 176-46 transition could be used. To date, however, we have not found it to be necessary to use both transitions for explosives detection.

As mentioned in previous sections, the ability to perform MS/MS on a selected ion from the mass spectrum of an explosive provides dramatically enhanced specificity in the analysis which helps minimize the potential for false alarms. For example, there is occasionally a background ion at $\mathrm{m} / \mathrm{z} 176$ which, of course, is the same mass/charge used to identify RDX. However, when MS/MS on this background ion is performed, the characteristic product ions for RDX $(\mathrm{m} / \mathrm{z}$ 102 and $\mathrm{m} / \mathrm{z} 46$ ) are not found; in fact the ion undergoes very little fragmentation. (This ion apparently tends to undergo electron detachment yielding no information as to its structure.) Thus, in cases in which this ion appears in the mass spectrum, a single stage of mass analysis might lead to the conclusion that RDX was present. However, the second stage of mass analysis can clearly establish that this ion is not derived from RDX. Our experience has indicated that this background species is most important when we sample packages and therefore may somehow be related to commonly used packing materials.

A second method for acquiring MS/MS data resembles the conventional method with the exception that the first quadrupole (Q1) operates as a high-pass filter (allowing only certain ions above a specified mass/charge value to pass). This is the "targeted product ion" moue [37] discussed in Section $V$. It is a "reduced specificity" form of MS/MS in which the parent-product ion relationship 
is not defined by the experiment resulting in loss in specificity relative to conventional MS/MS scan. However, one advantage of this method is increased transmission of parent ions. This mode of operation is useful for compound classes that yield a common product ion upon collisional activation. For example, $\mathrm{NO}_{2}{ }^{-}$is a characteristic product ion of nitroaromatics and $\mathrm{NO}_{3}{ }^{-}$is a characteristic product of nitrate esters. In the case of TNT, the $\mathrm{NO}_{2}$ ion would be the targeted product ion. To take full advantage of this application, the $\mathrm{NO}_{2}$ signal in the MS/MS spectrum of M of TNT was maximized. This was done by experimentally determining the optimum collision energy for $\mathrm{NO}_{2}$ production. Figure 27 shows this energy resolved (ERMS) plot acquired with 20\% parent ion beam attenuation. Under these conditions, laboratory collision energies $\left(E_{\mathrm{lab}}\right)$ around $40 \mathrm{eV}$ provide the best compromise between transmission and $\mathrm{NO}_{2}$ production. A variation of the "targeted product ion" mode is to scan over a narrow $\mathrm{m} / \mathrm{z}$ range (to improve duty cycle). Examples of the targeted product ion spectra are given in Figure 28.

Note that the energy-resolved plot of Figure 27 can provide enhanced informing power, or specificity, if needed. This point was discussed briefly in Section IV. Even in cases in which the same mass/charge values are observed for both parent ions and product ions of two different species, these species may be distinguishable based on their energy-resolved MS/MS spectra. The variation in product ion relative abundances as a function of collision energy or pressure may provide the necessary distinguishing behavior. This approach adds the internal energy-dependent behavior of an ion to the criteria for identification. This approach is most often required in distinguishing isomeric species and is not expected to be necessary in the explosives detection scenario. However, if desirable, it is likely that various substituted nitroaromatics could be distinguished on this basis.

\section{Sensitivities in Various Operating Modes}

On the triple quadrupole system, the relative sensitivities for explosives in the various operating modes are substantially different. On a relative scale, the 


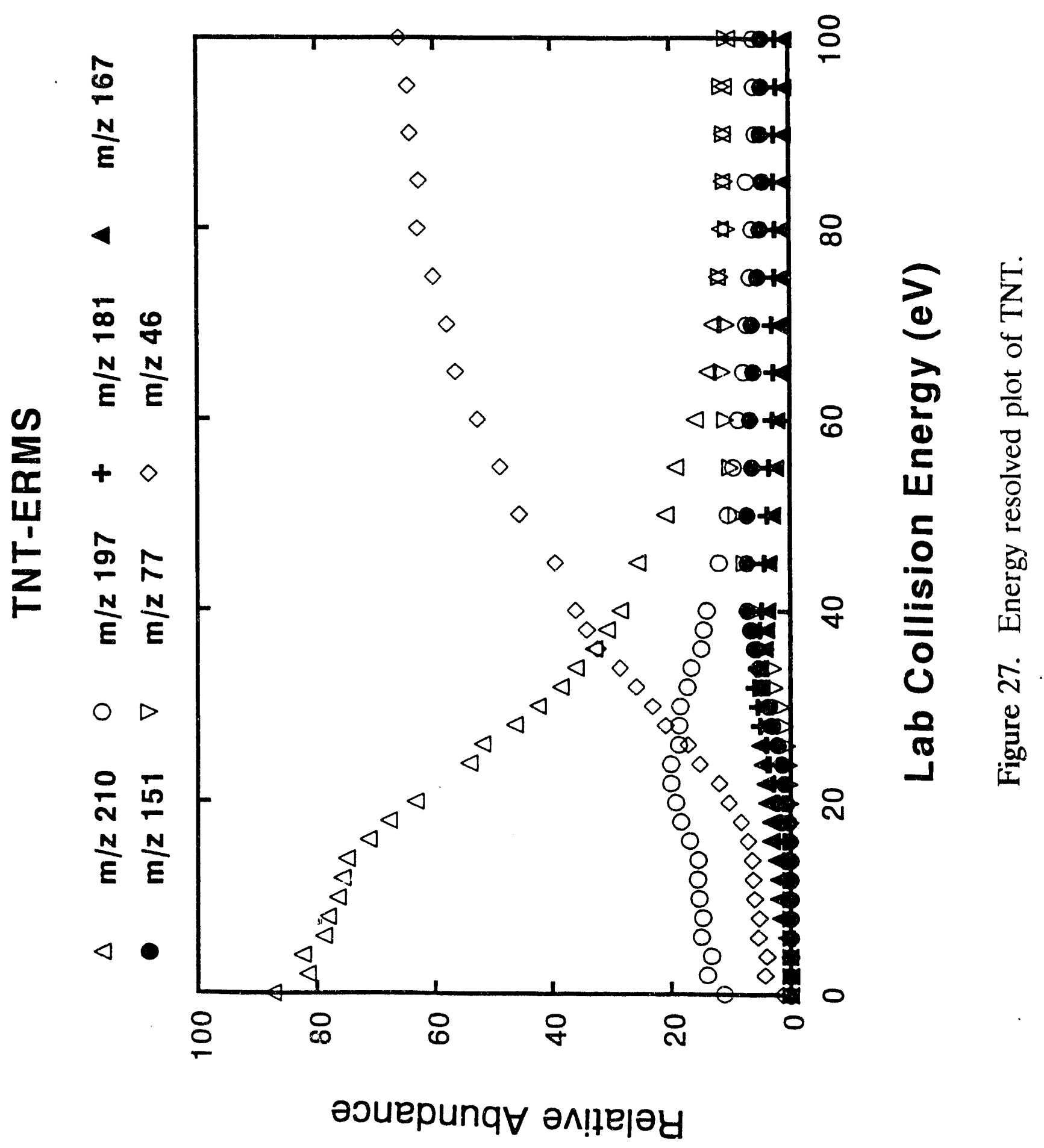


83
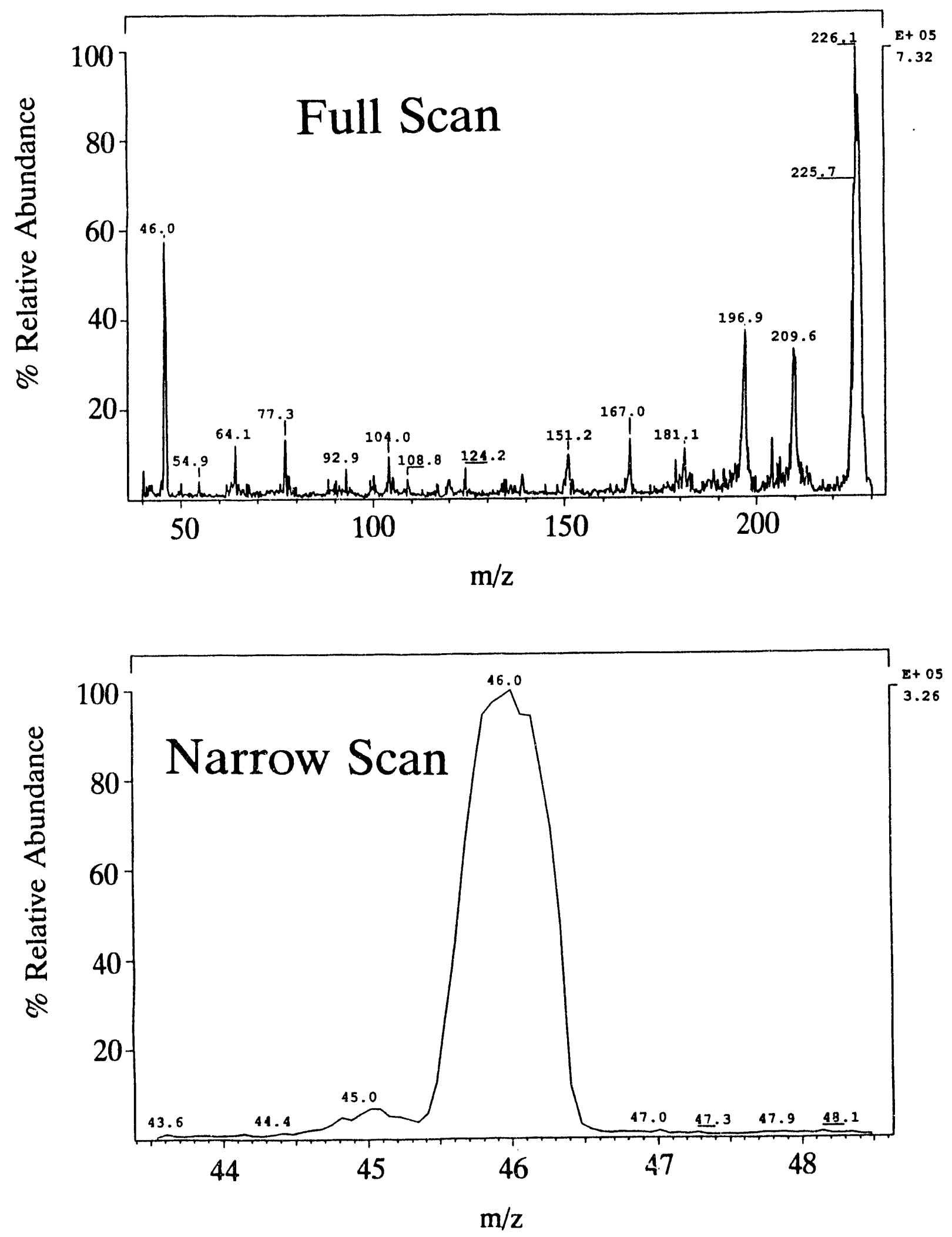

Figure 28. Targeted product ion mass spectra for INT. 
sensitivity of the various modes of operation are in order of decreasing (best to worst) sensitivity:

\section{MS > TARGETED PRODUCT > MS/MS}

In the standard full scan MS mocie, when the second quadrupole (Q3) is scanned, we can inject ten picograms of an explosive (TNT or RDX) and detect a signal associated with the explosive $(\mathrm{m} / \mathrm{z} 227$ or $\mathrm{m} / \mathrm{z}$ 176). At these injection levels with full scan, it is difficult to see the ion in the total ion chromatogram due to the large contributions of the normal background ions, but when the chromatogram is reconstructed to plot only the ion of interest, the signal/background is good. The levels injected correspond to explosives at the sub-ppb level in air. Of course, single ion monitoring provides much lower detection limits due to the $100 \%$ duty cycle (see Section IV). Detection limits for RDX of $400 \mathrm{fg}$ in air, for example, were measured with our original quadrupole/time-of-flight instrument operated in single ion monitoring mode [66]. Sub-picogram detection limits are also expected with the TSQ 700 operated in this manner.

However, when going to the MS/MS experiment in a beam-type instrument, various parameters come into play which affect the sensitivity, such as transmission losses, fragmentation efficiency, and collection efficiency (see also Section IV). First, the transmission of ions through the first quadrupole is reduced when going from an rf-only mode to an $\mathrm{rf} / \mathrm{dc}$ mode. Second, scattering losses in the collision ragion where the analyte ions undergo collisions with the neutral target reduces transmission of ions into the second quadrupole. Thus, greater levels of analyte would be required in order to obtain meaningful data. Typically, detection limits on the TSQ 700 in MS/MS mode are about ten times worse than MS.

In the targeted product ion mode the first quadrupole operates in rf-only mode, which increases ion transmission, giving better sensitivities than standard MS/MS conditions. However, the sensitivity does not approach MS sensitivities because there is still ir?efficient collection of product ions and small transmission losses from the collision region to the second quadrupole. In the ion trap, however, the sensitivities between MS and MS/MS do not differ appreciably 
because there are essentially no transmission losses and the collision process is very efficient (see Section IV).

\section{Automated Explosives Detection}

Automated monitoring systems for identifying the presence of toxic or controlled substances are of great importance in many government and industrial applications. Minimal user interaction and technical skill are required in many cases for complete automated analysis of gaseous or liquid samples. This section addresses the development of an instrument control application with a simple user interface to run the TSQ 700.

The Finnigan TSQ 700 is a highly computerized mass spectrometer with very few manual dials for control of instrumental parameters. There are only three manual dials, two buttons and four display panels on the unmodified instrument. A picture of the TSQ 700 with the ASGDI modification and the associated work station is provided in Figure 29. The three dials control needle valves that regulate flows of the calibration, chemical ionization, and CID gases into the instrument. The pressure measured in the source and collision cell regions of the instrument are sent to the two displays on the right side of the front panel of the instrument. The other two display panels on the left side of the instrument are used to monitor the detector. Finally, one of the buttons provided on the front of the instrument is an emergency shutoff switch that was included mainly for safety while the other controls the "ball valve" inlet system on the unmodified instrument (the ball valve assembly was replaced with the ASGDI source inlet). Thus, there is no way of controlling the instrument from the front panel. Instrument control is accomplished entirely via software on the data system. While this arrangement may seem to be a disadvantage to those who prefer to manipulate knobs manually, it has many advantages for a triple quadrupole mass spectrometer (e.g. the ability to scan voltages applied to lens elements during a mass spectrum scan to optimize transmission across the entire scan) and is absolutely essential for developing an automated explosives detector based on this instrument. 


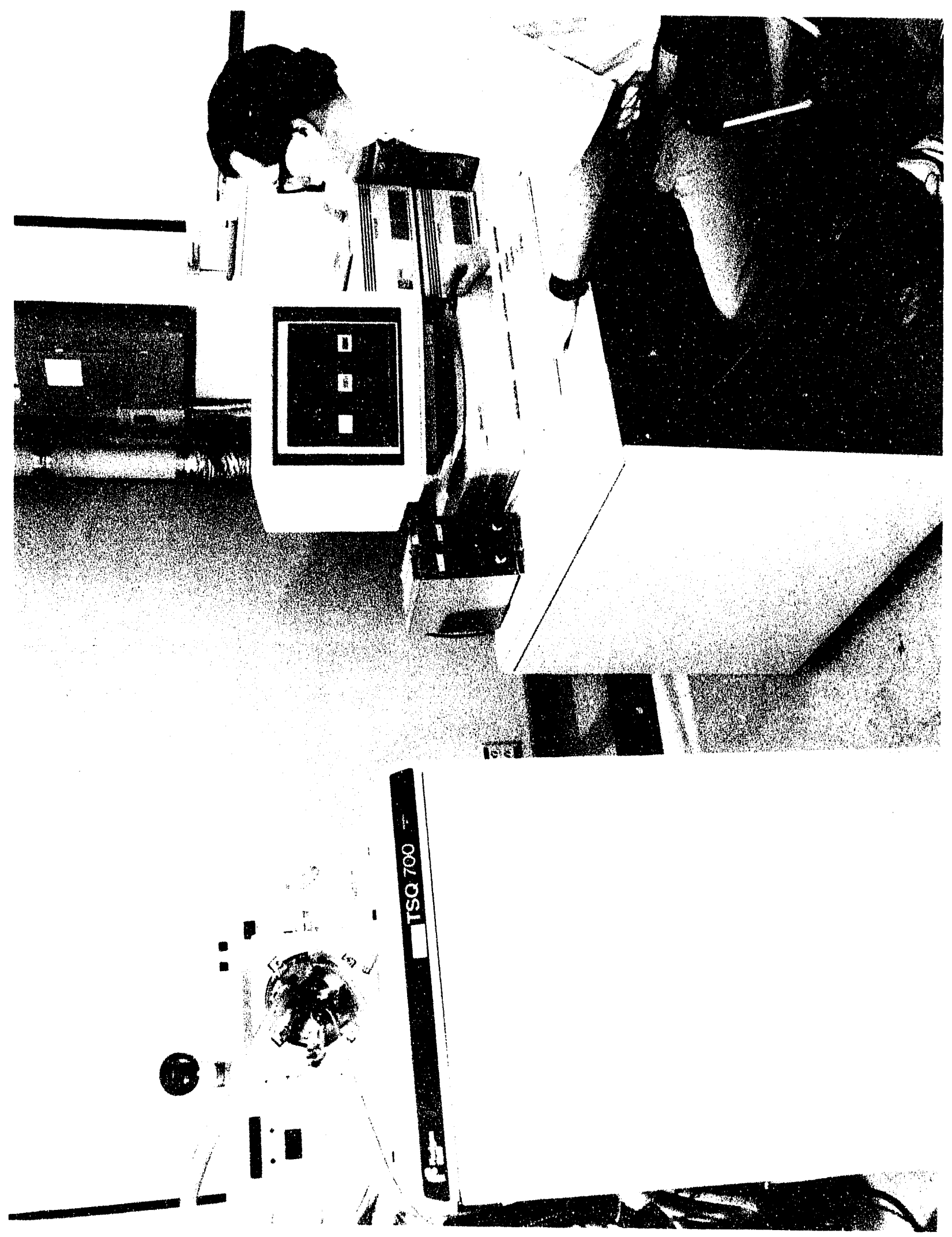

Figure 29. Photograph of the TSQ 700 with ASGDI source assembly and associated work station. 
There are two kinds of software on the TSQ 700 . Instrument control software is loaded into "read-only memory" (ROMs) on three Finnigan Single Board Controllers (FSBCs) that are located at the left lower rear area of the instrument. The FSBC's are the Instrument Control (IC) FSBC, Display Control (DC) FSBC and Real-Time Interface Control (RC) FSBC. The software is executed by CPU's located on these boards. This software, referred to as "firmware", is loaded from the hard drive of the data system (shown on the right in Figure 29) when the instrumental control window is invoked. Data analysis and manipulation software applications are stored on the hard drive of the data system and are executed by the DEC workstation. This software is referred to as ICIS ${ }^{\text {TM }}$ (Interactive Chemical Information System). The physical link between the on-board CPU's and the data system is an ethernet connection.

The instrument control window is invoked by selecting the TSQ 700 menu selection from the ICIS Executive Application list. A series of instrument control programs (i.e. firmware programs) are run within this window. For example, the most often used instrument control windows are: TUNE (provides a means of automatically optimizing voltages applied to various components of the mass spectrometer and viewing the results), QUADS (shows the current scan mode and instrument status), VALVE (shows instrument pressures and valve and pump status), PROF (displays profile mass spectra for each scan of the instrument) and ANALYSIS (allows spectra to be stored to disk and the integration of GC and instrument control procedures). A screen snapshot of these windows is provided in Figure 30. The status of the instrument is continually updated in these windows. The use of these windows is the subject of several chapters in the TSG 700 documentation. These views can be displayed simultaneously within the instrument control window or expanded to full size for better viewing. The TSQ700 documentation cites 33 different views and can be grouped according to the following categories: Display Control, Inlet Control, Mass Spectrometer Control, Mass Spectrometer Status, Data Acquisition, Data System Display, ICL Utility, Genera! Utility and Diagnostic. 


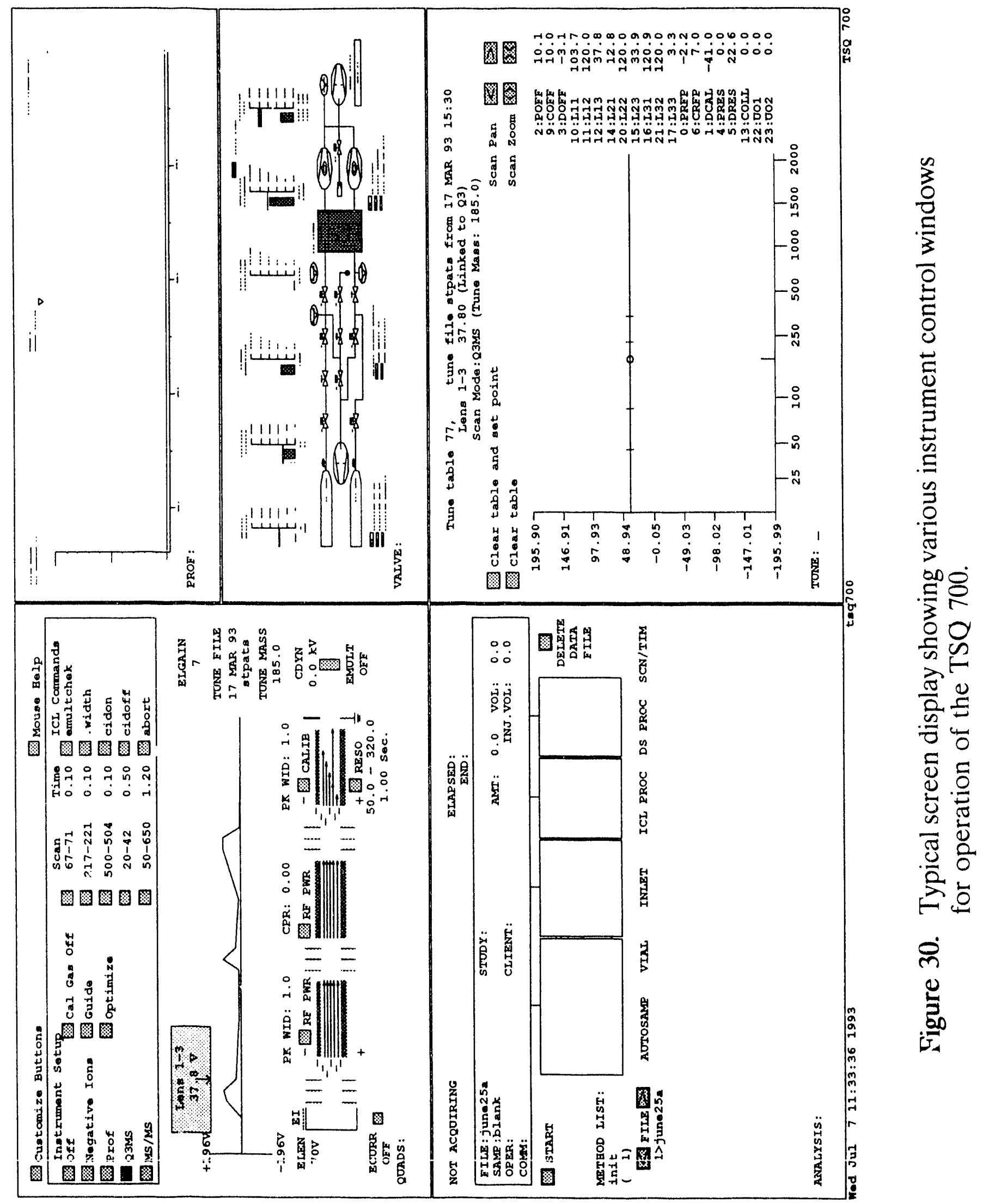


There are different classes of commands that are used to control the TSQ700 and the display windows but the class that is most relevant to this discussion are ICL commands. These commands are often referred to as "dot-commands" because they are preceded by a period (e.g. ".q1ms"). These commands can set a value (e.g. ".emult 1500" sets the electron multiplier to $1500 \mathrm{~V}$ ) or perform. a task (e.g. ".cgon" opens the solenoid valve controlling the calibration gas). The ICL language contains key words similar to those found in BASIC or C that can be used to create conditional statements and loops. Most important to the development of an automated explosives detector, these key words can be combined to create instrument control procedures, hereon referred to as "ICL procedures".

\section{ICL Procedures}

An ICL procedure is a set of ICL commands that have be $n$ jentered into an editor and saved to the disk under a specific filename (*.ICL). The EDIC instrument control view is used to create these files. Once an ICL procedure has been created and saved to the hard drive, it can be invoked as an ICL command (i.e. a dot command). There is a large directory of $I C L$ procedures supplied by Finnigan to control the instrument and these may be called within user defined ICL procedures. The snly difference is that the dot is not used within an $\mathrm{iCL}$ procedure.

As noted in the ICL help display on the data system, ICL keywords are dividad into seven classes. Thase classes are Paramieters, Tables of Parameters, Commands, Feedback words, Command / Feedback words, Procedures and Structure words. Parameters are used to set instrument variables (e.g. $F M=150$ means set the first scan mass to 150). Tables of parameters deal with collections of data such as user lists (e.g. $X=\operatorname{ULIST}(32,2)$ where ULIST refers to the user list that resembles a spreadsheet) and tune tables (e.g. $L 11(5)+.=1$ ). User lists are useful for storing, listing and manipulating sets of numbers. The designation $L 11(69)$ in the example above refers to the voltage stored in the tune table for lens 
1-1 at $\mathrm{m} / \mathrm{z} 69$ (leris voltages can be scanned during the acquisition of a mass spectrum). Commands initiate an action of some kind or perform a task and may have a set of arguments (e.g. GO 100,150,.1). In this example, one scan is performed from m/z 100 to $\mathrm{m} / \mathrm{z} 150$ in 0.1 second. Feedback words can receive input from sensors or calculations (e.g. IF ISPR > 10000: \# Pressure too high!). The sensor monitoring source pressure is accessed by the keyword ISPR as shown in the example above and a decision can then be made. Command / Feedback words return a value if used in an expression or perform a task if used by themselves (e.g. "CENT" makes the data system display centroid spectra while CENT in the expression "IF CENT: PLOT(RT,AREA) : ELSE: PLOT(RT,HEIGHT)" returns either true or false. The category labelled Procedures is somewhat confusing since procedures can function as cormmands or feedback words. The distinction appears to be that a top level procedure is considered a "Procedure" while any procedure called within that procedure becomes a "Command" or "Feedback" word. Finally, structure keywords (e.g. if, else, do, repeat, etc.) are used to bind together commands and feedback words into procedures.

Two examples of ICL procedures that were provided with the TSQ 700 are shown in Figures 31 and 32. The "ON" procedure shown in Figure 31 is useful when operating the instrument in electron ionization (EI) mode. This procedure turns on the filament in the El source, the electron multiplier and the conversion dynode of the unmodified instrument. This example illustrates using feedback to make decisions. The second procedure shown in Figure 32 simply illustrates the use of other procedures as commands within a new procedure (i.e. the command SETUP is a procedure stored on the hard disk as "SETUP.ICL" and similarly for TUNEQ1 and TUNEQ3). The ability to create these procedures with a high degree of computer control over instrumental parameters was critical to the successful development of an automated explosives detector using a triple quadrupole mass spectrometer. 


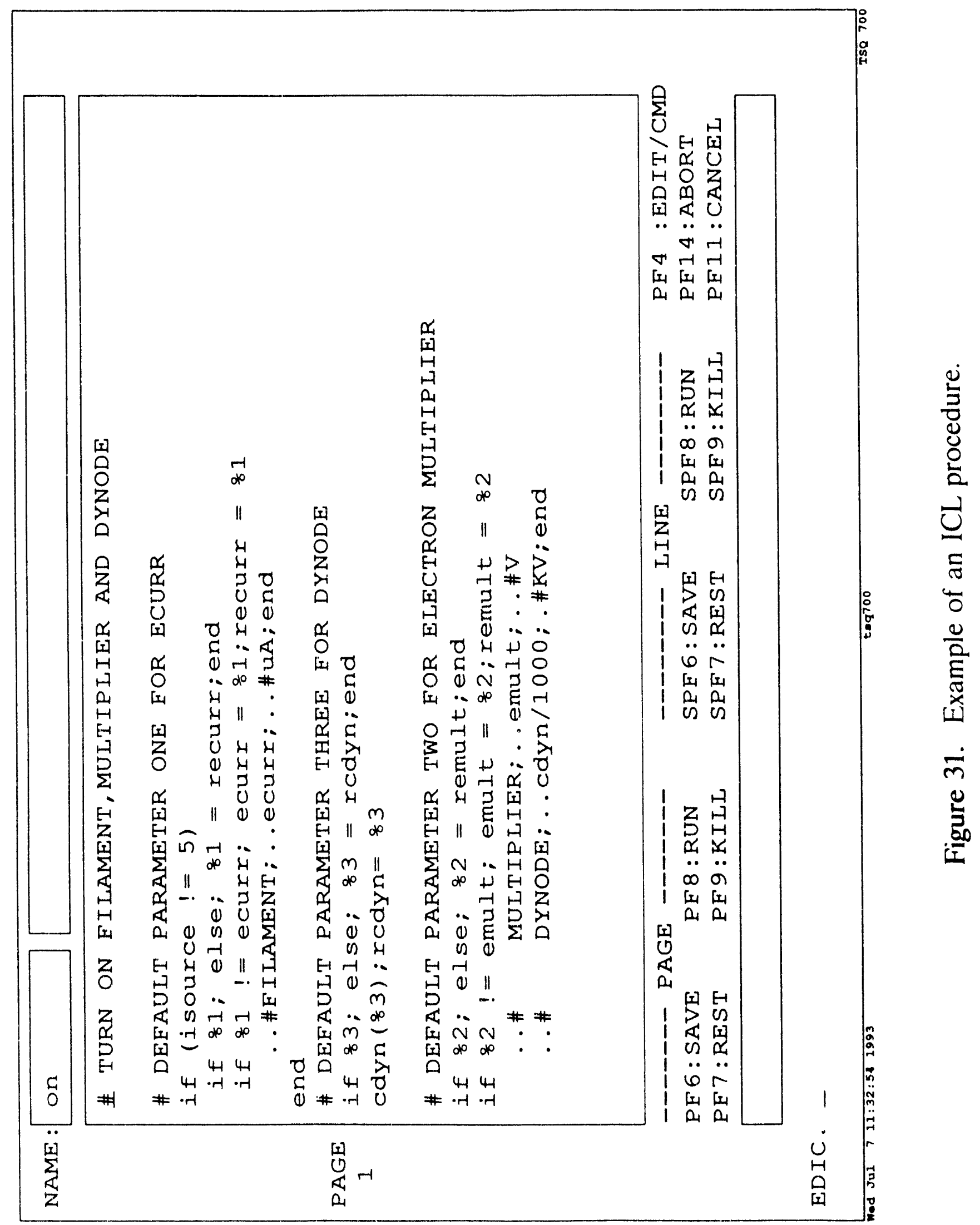




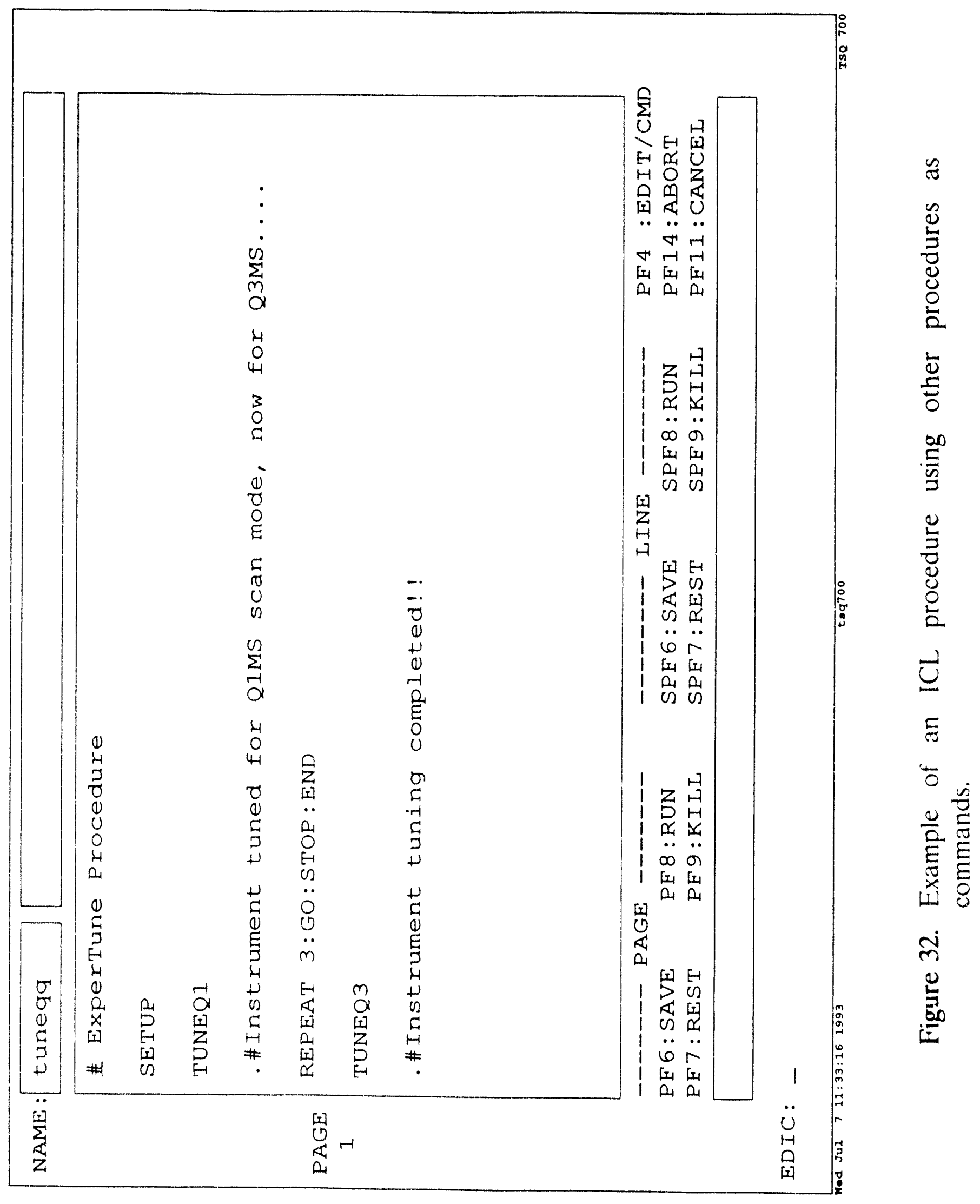


2. Automated Explosives Detection Procedures

Simple user interaction and display of results were the principal software design considerations for the development of an automated explosives detector. Instrumental and sampling considerations have been discussed elsewhere. The ICL procedures discussed in the previous section and a "GUIDE" window added in the last TSQ-700 software update allowed for a very simple push-button sensor application to be developed. A listing of each of the ICL procedures that comprise the automated explosives detector software is provided in Appendix II.

The guide window was added by Finnigan to allow commands to be associated with icon "buttons" displayed on the screen of the data system as shown in Figure 33. Users can then select actions listed on each button rather than trying to operate the instrument through the more complicated windows shown previously in Figure 30. An option is selected by clicking on the button with a mouse. Once a button has been selected an associated ICL procedure is executed. There can be nine buttons displayed in the guide window at one time. The button command consists of a series of digits followed by ".\#<button label>" where the button label is a short text description displayed inside the square button. A longer text description can be included and is displayed above the button. The last item in the button command is the name of the ICL procedure to be executed if the button is selected. An example of using the button commands can be found in the "BUTOP" ICL procedure in Appendix II.

A screen dump of the main menu of the ORNL Explosives Detector application is shown in Figure 33. This menu was initiated by executing the BUTOP ICL procedure and was displayed in the GUIDE window. This window can be expanded to fill the entire screen as shown in Figure 34. There are three options in this first screen: 1) TUNE, 2) START and 3) RESET. The new button ICL commands also support color so each button can be assigned a different color. The first option calls ICL procedures to tune the instrument for optimal performance and calibrates the mass scale. Most of these procedures were 


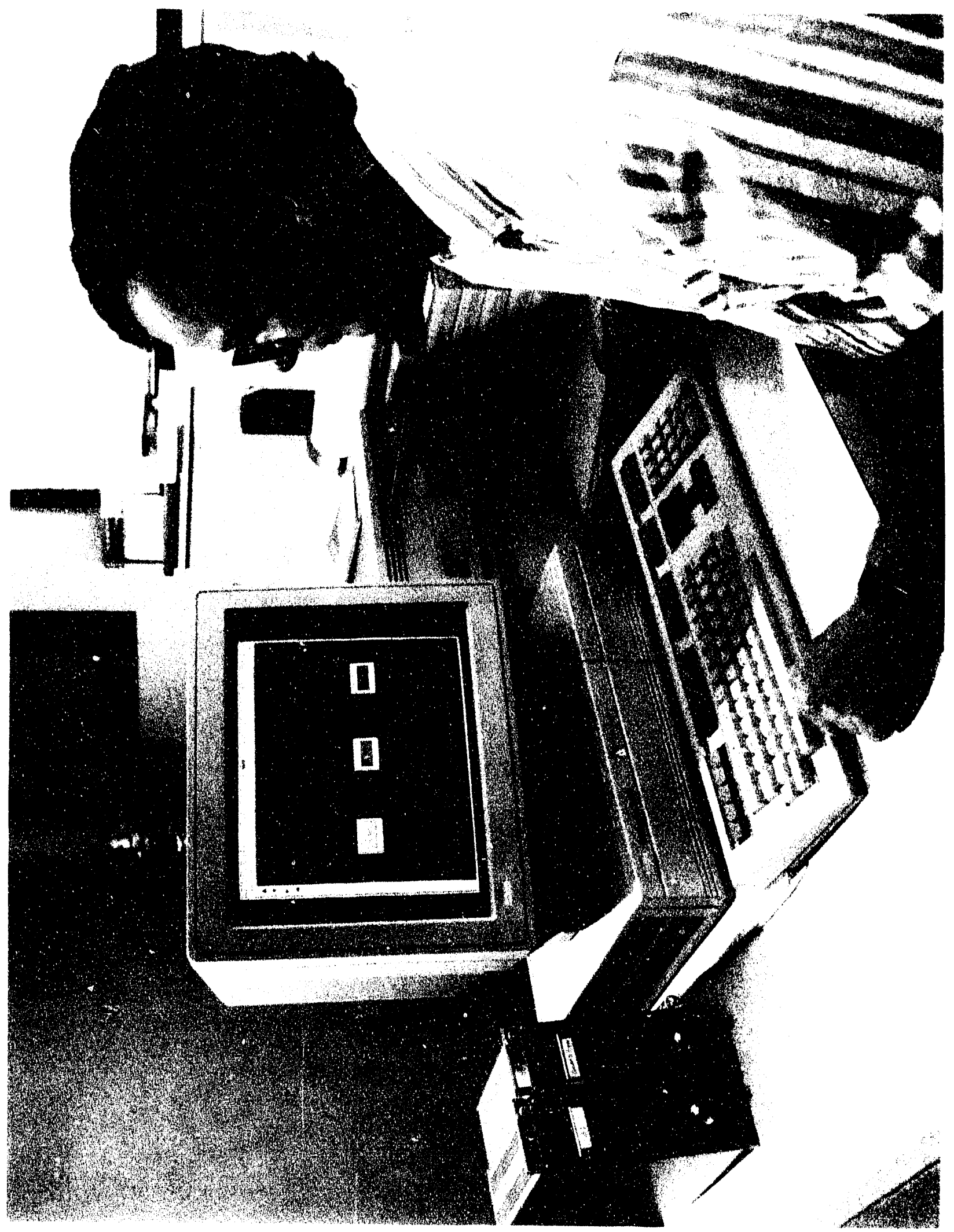

Figure 33. Guide window showing icon "buttons" allowing simple push-button operation. 


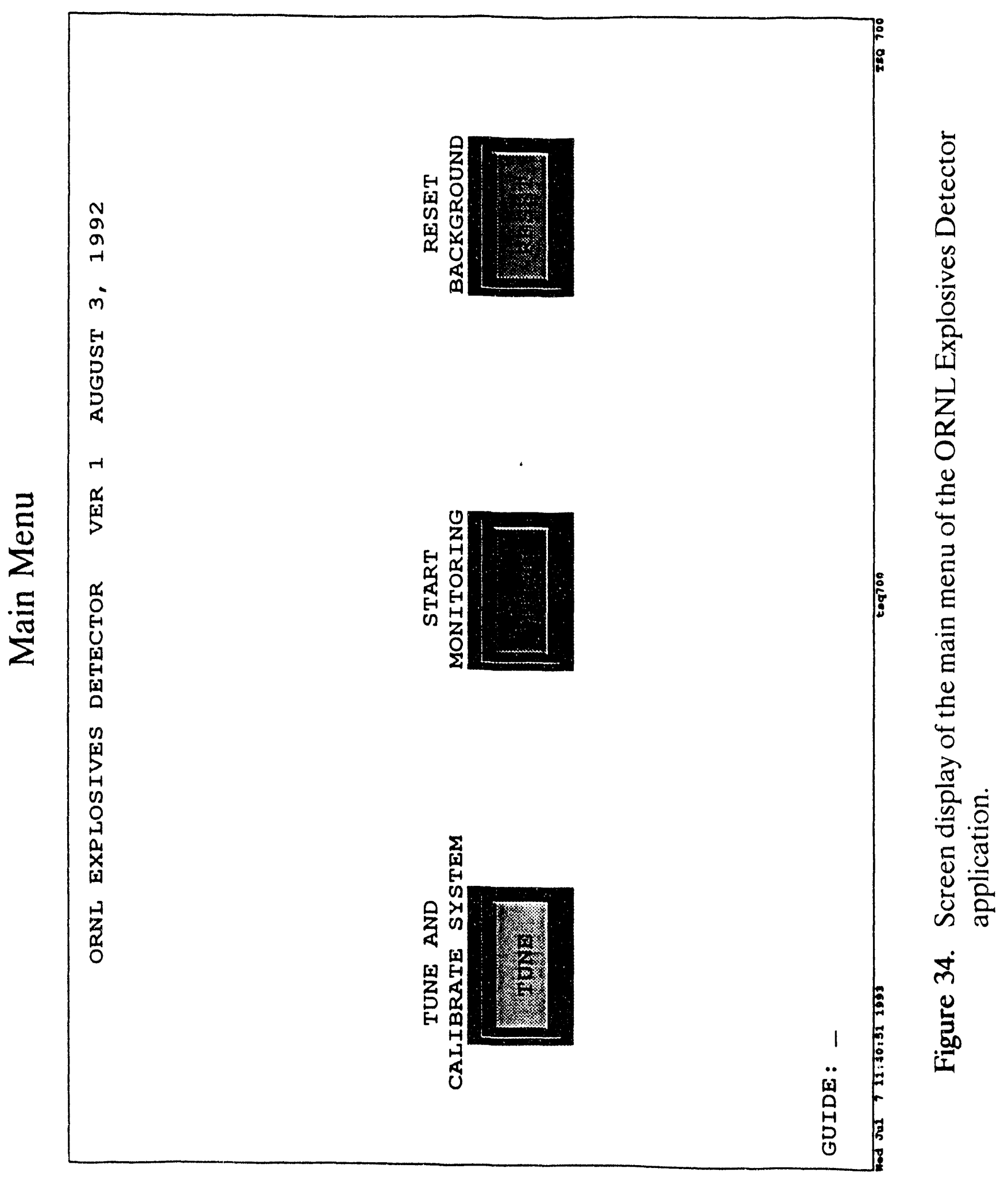


provided with the instrument and only minor changes were required to support the use of trifluoromethylcyclohexane as a tuning and calibration compound instead of perfluorotributylamine. Trifluoromethylcyclohexane was used because it provided better spectra under the ASGDI conditions used for explosives detection. The second option calls a set of procedures which continually acquires mass spectra and monitors for a significant ion abundance at selected $\mathrm{m} / \mathrm{z}^{\prime} \mathrm{s}$. The third option characterizes background ion intensity at the selected $\mathrm{m} / \mathrm{z}^{\prime} \mathrm{s}$ and resets the values used when the second option is selected. This option is automatically executed when the tune and calibrate option is selected.

The monitoring option represents the bulk of the automated explosives detector software. The ICL commands provide a means of accessing spectra immediately after acquisition so on-line analysis of the spectra is possible. A method to continuously acquire MS spectra using the ASGDI ion source and monitoring the spectra for ions with $\mathrm{m} / \mathrm{z}$ 's corresponding to potential parent ions of selected explosives has been implemented. A flow chart of the algorithm used is provided in Figure 35. The screen displayed to the user is split when the monitoring mode is selected as shown in Figure 36. The GUIDE window is reduced in size and resides on the left side of the screen. An "ALL CLEAR" message is displayed at the top of this screen if no selected ions have been detected above background. A stop option is included at the bottom of this window. The profile MS spectrum obtained is displayed on the right side of the screen. If one of the parent ions of the selected explosives is detected, the user is alerte ${ }^{-1}$ hy a message in the GUIDE window (i.e. "!! WARNING !! POSSIBLE EXPLOSIVE !! WARNING !!") as shown in Figure 37. A reset option is also provided. The corresponding profile mass spectrum is displayed in the PROF window on the right of the screen.

Confirmation of the presence of one of the targeted explosives is obtained by automatically switching the instrument into MS/MS mode and acquiring a CID product spectrum. The MS and MS/MS spectra obtained for TNT, PETN and RDX were discussed in sections VI.A and VI.B. If the presence of one of these 


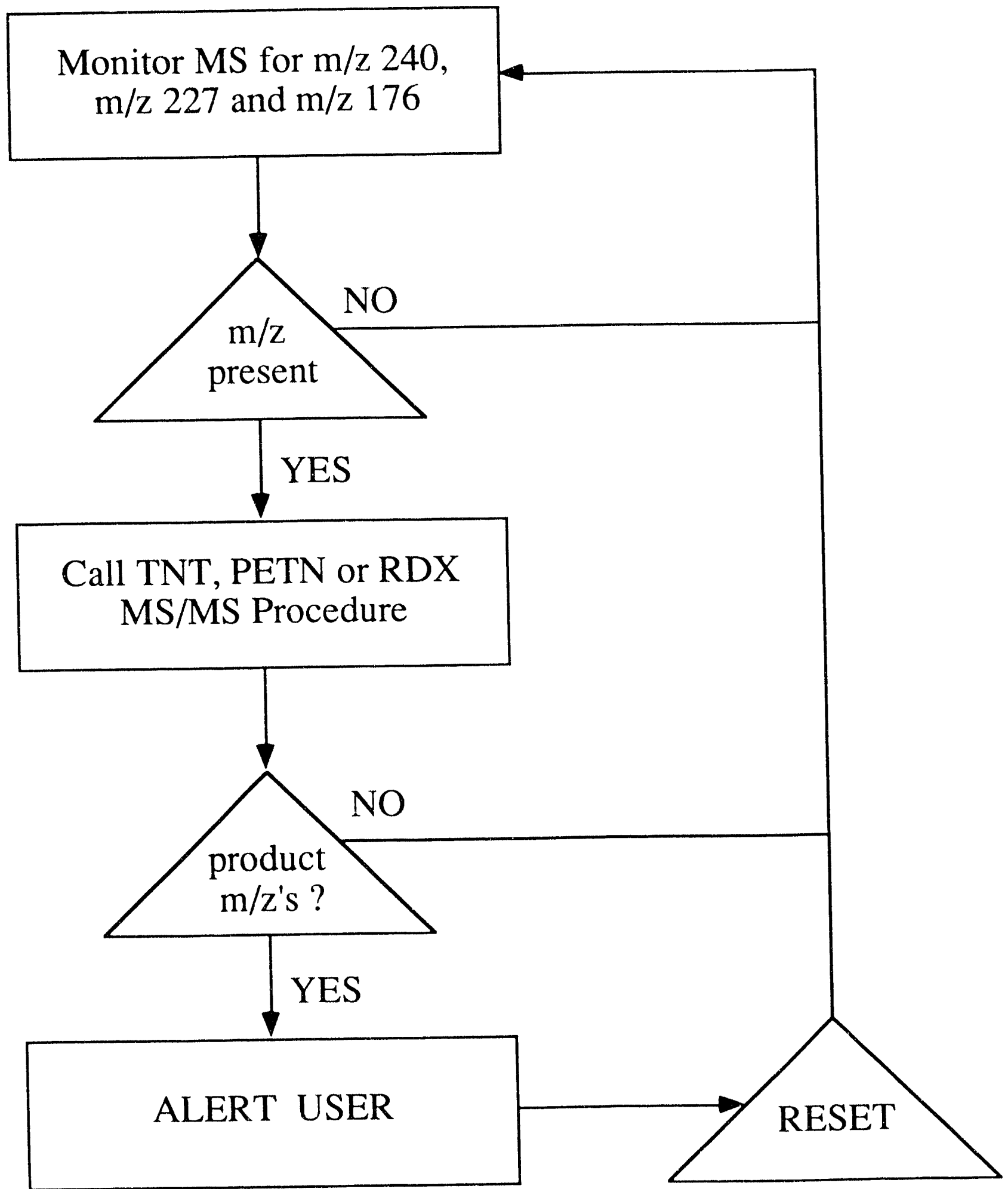

Figure 35. Flow chart of algorithm used for automated explosives detection on the ASGDI/TSQ70). 


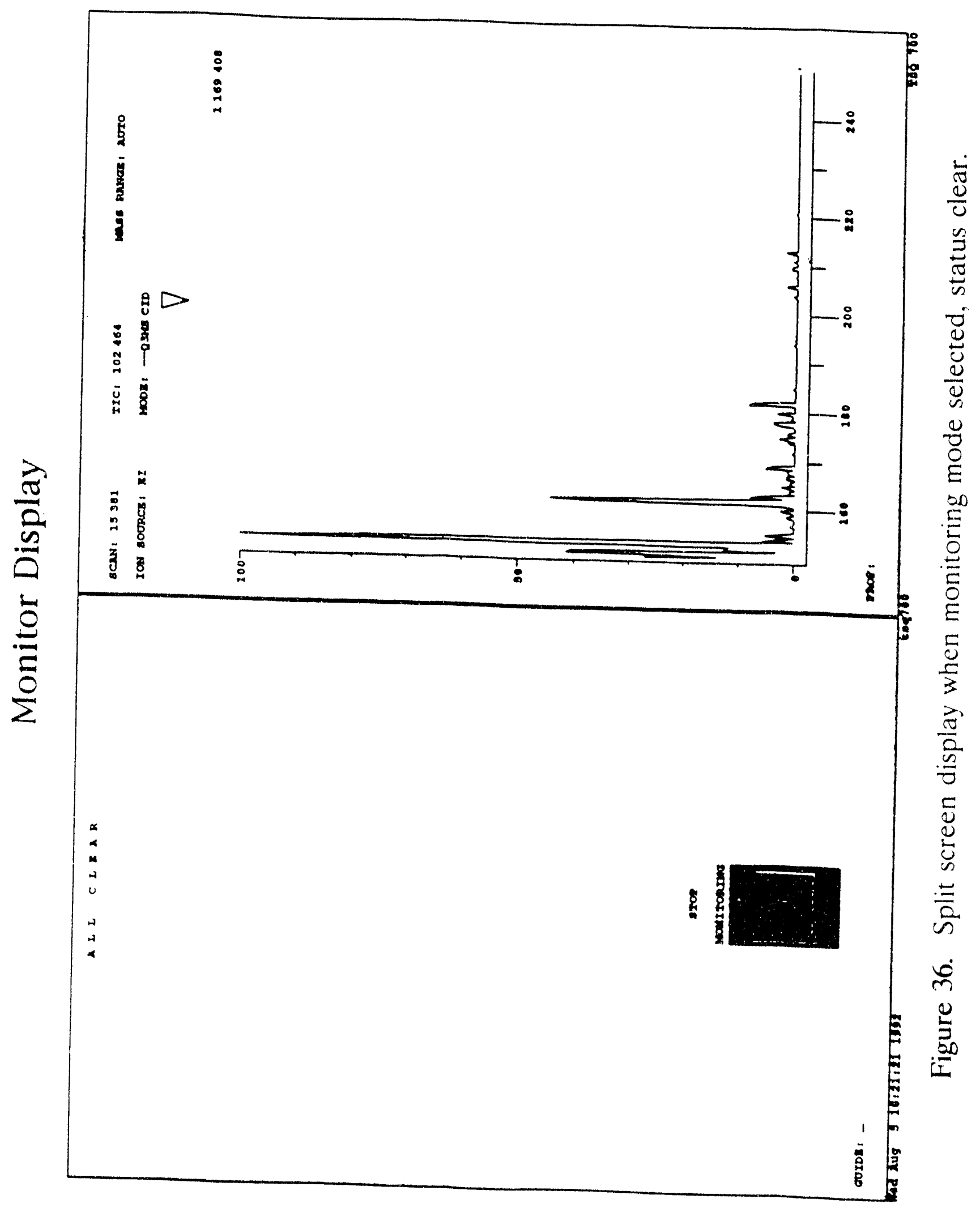




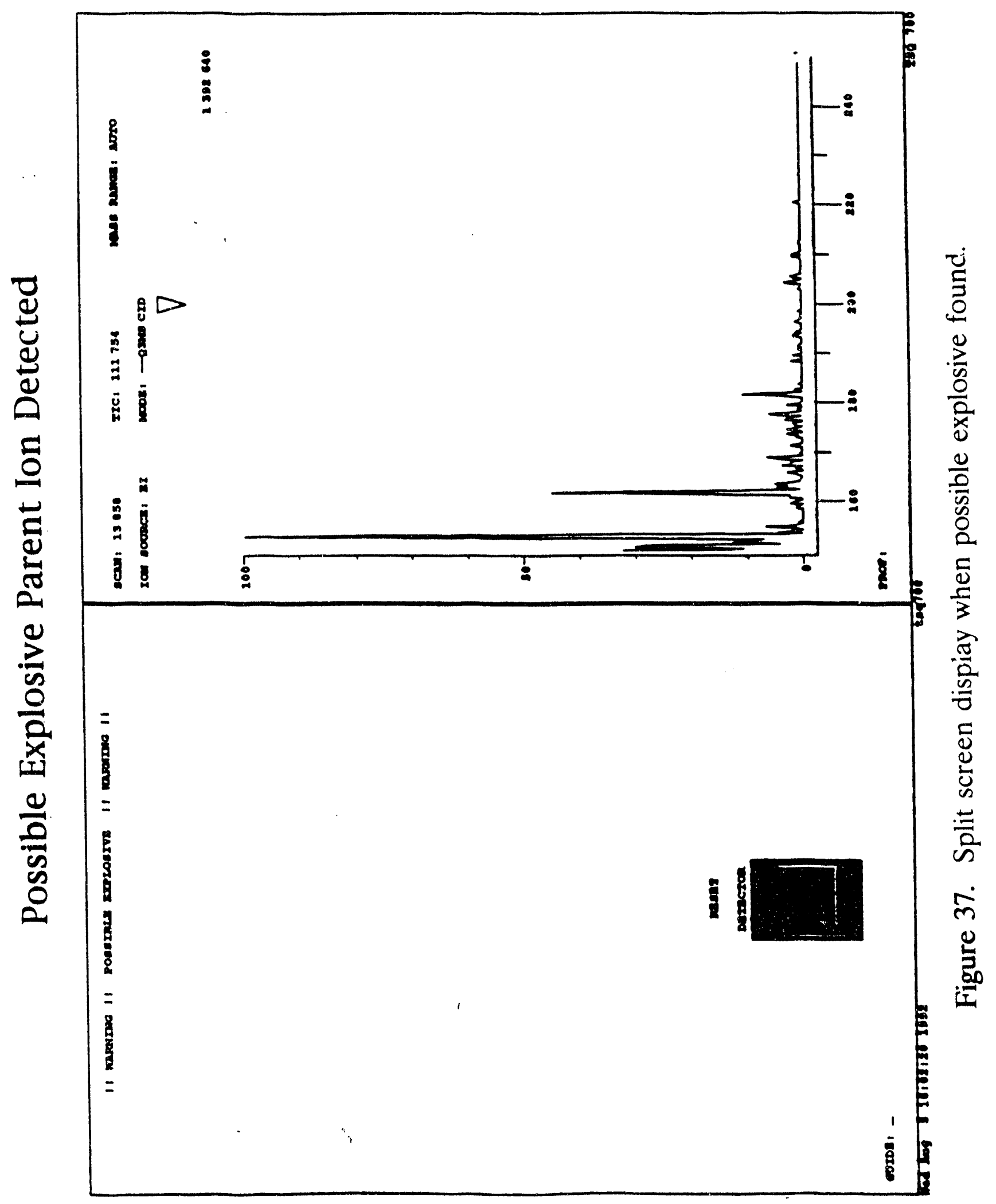


explosives is confirmed by the presence of diagnostic product ions in the MS/MS spectrum, the user is alerted in the GUIDE window (i.e. "!! ALERT !! EXPLOSIVE DETECTED !! ALERT !!") and a signal is sent to a user output located on one of the TSQ 700 electronics boards. This signal drives a flashing red light located on the top of the instrument. The alert screen is shown in Figure 38. The MS/MS spectrum acquired is displayed in the PROF window on the right of the screen. A snapshot of the screen is also sent to the printer for a hard copy record.

Chemical background signals are measured at the selected $\mathrm{m} / \mathrm{z}^{\prime} \mathrm{s}$ in the $M S$ and MS/MS spectra by using the reset background option. The background abundances measured using the "BOOMBACK" procedures included in Appendix II are stored in a user list. The background abundance stored in the user list is five times the actual background averaged over 10 scans. Thus, a signal-tobackground ratio $(\mathrm{S} / \mathrm{B})$ of 5 is used as a decision threshold for determining the presence of selected parent and product ions in the spectra being monitored. The largest contributor to chemical noise in the MS spectra is the normal background associated with the ASGDI source. Field testing of the instrument or a specific effort to simulate background chemicals encountered in the field is required to address the effect of these chemicals on the automated explosives detector. However, chemical background is minimal in MS/MS spectra unless there is an isobaric parent ion that produces the product ions being monitored, a situation not yet encountered. Electronic background or noise is zeroed during tuning. 
101

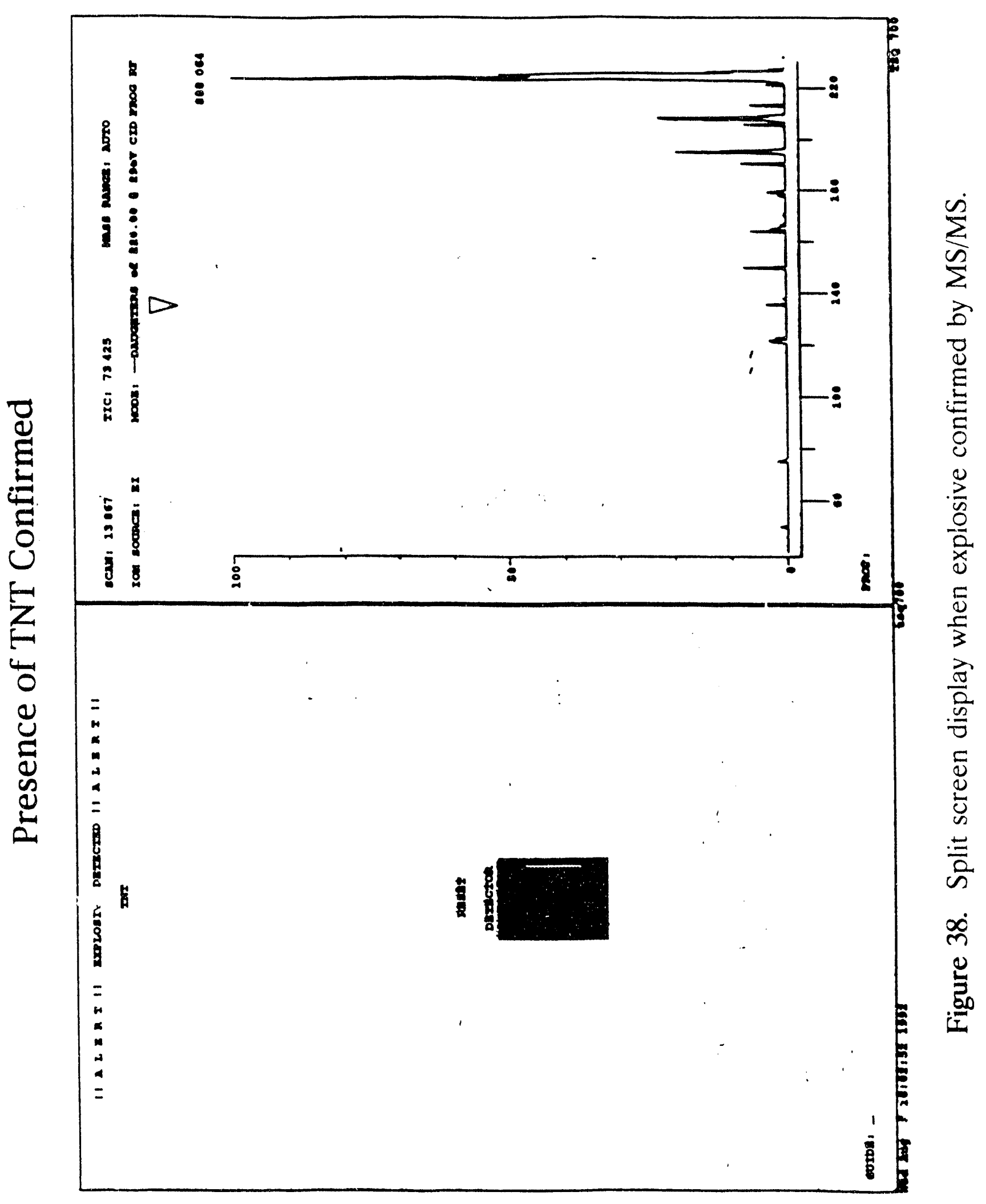




\section{COMPARISON OF ASGDI ON VARIOUS TANDEM MASS SPECTROMETERS}

Section IV emphasized generic differences and similarities in various approaches to tandem mass spectrometry. At ORNL, we have three instruments capable of MS/MS coupled with ASGDI. We have therefore performed bench-mark experiments to provide for quantitative comparison of the different MS/MS geometry instruments. The comparison involved the ASGDI/TSQ 700, as described in the previous section, an ASGDI/ion trap system, and an ASGDI/quadrupole/time-of-flight system. The latter instruments are described briefly below.

Quadrupole ion trap technology for tandem mass spectrometry was described in Section IV.B under the category of ion trapping instruments. As mentioned in that section, the ion trap is not well suited to forming anions by conventional in-situ ionization. We therefore coupled an ASGDI source with an ion trap [60] as shown schematically in Figure 39. The rationale behind the use of ASGDI with the ion trap is to form the anions in an environment with a large number density of slow electrons rather than in the ion trap itself where there is a low number derisity of slow electrons. The anions that issue from the ASGDI source are focussed onto the aperture of the entrance end-cap during the ion accumulation step by the three-element lens system indicated in the figure. At all other times, ions are deflected from the entrance end-cap by the central lens element, which is comprised of two semi-circular half-plates to allow for ion deflection. A key to the successful mating of external ion sources with an ion trap is the efficiency of ion injection. Ion injection is facilitated by the presence of helium in the vacuum system at a pressure of about one millitorr. Collisions with helium constitute a damping factor that enhances the probability that an ion injected from an external ion source is trapped. In our system, we estimate an ion injection efficiency of $1-5 \%$. 


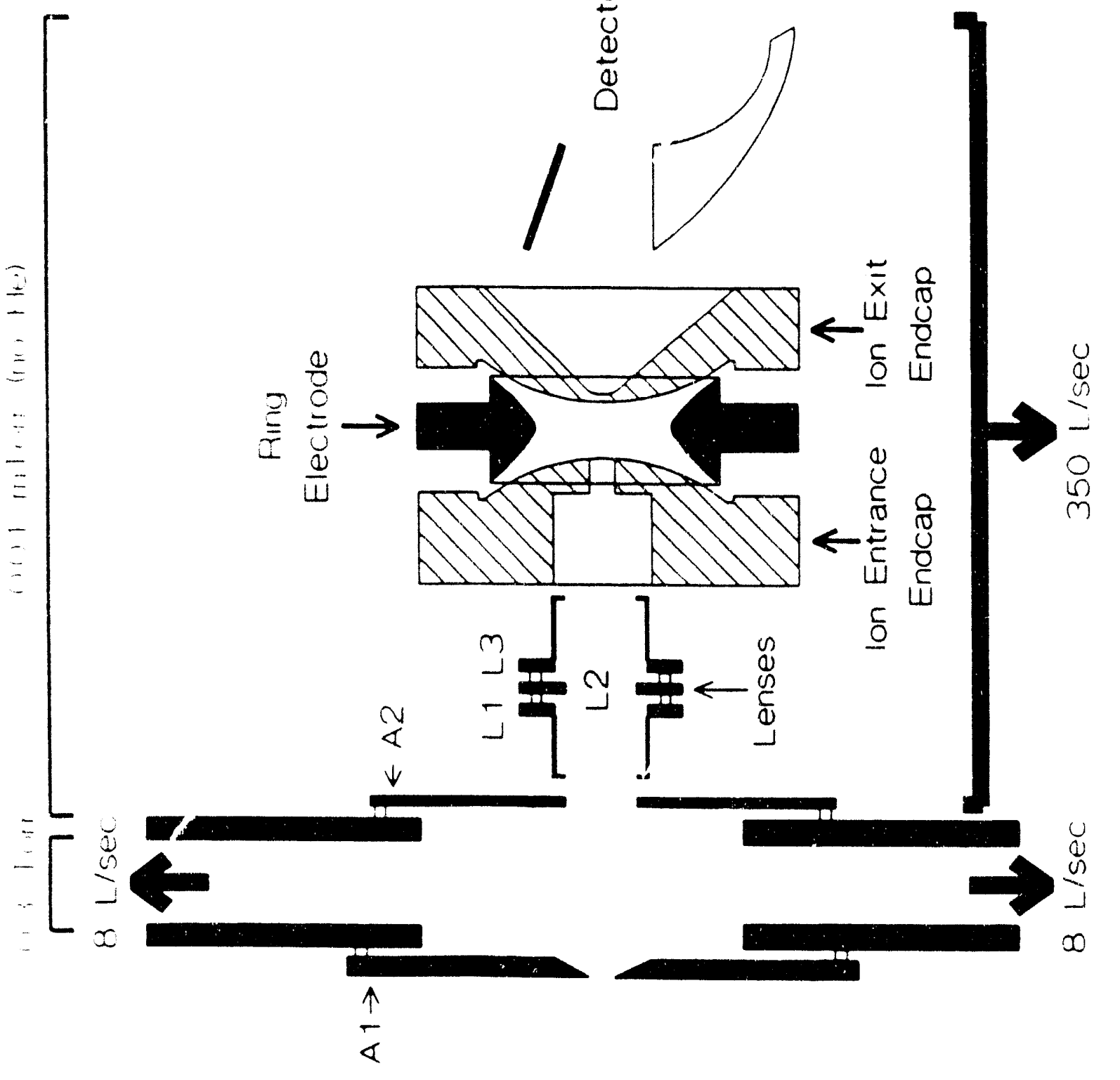

$\stackrel{0}{ \pm}$
$\frac{5}{5}$

$\frac{8}{8}$

苛

$\overline{1}$

它

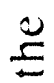

$\bar{\Xi}$

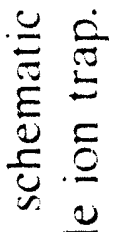

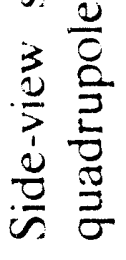

is

冚 
The quadrupole/time-of-flight (QT) instrument was the first tandem mass spectrometer coupled with the ASGDI source for explosives detection $[66,67]$. A schematic diagram of this instrument is shown in Figure 40. The QT instrument is , of course, a beam-type instrument which is essentially identical to the TSQ 700 up to the collision region. The collision region in the QT is a small gas-filled chamber with entrance and exit apertures for the beam and ports for admitting collision gas and for measuring pressure. Unlike the octapole collision cell of the TSQ 700, there are no external fields present to minimize scattering losses. The other major difference between the two beam-type instruments lies in the second mass analyzer, viz. a quadrupole mass filter versus time-of-flight. The time-of-flight portion of the instrument consists of a linear drift region of 1 meter. The ions are gated into the drift tube by a set of deflection plates mounted on the last plate of the acceleration lens assembly (see Figure 40). After transit though the time-offlight region, the ions are detected with a conversion dynode/electron multiplier detector identical to that used with the ion trap system. Because the ion energy is in the tens of electron-volts range for analysis by the quadrupole, and in the kiloelectronvolt range for time-of-flight analysis, one section of the instrument must be floated at high voltage by enclosing the drift region with a screen, electrically isolated from the vacuum hardware. Thus, the source and the quadrupole are operated at near ground potential. The quadrupole, collision cell, and lens system are housed in a six-way cross with ten inch Conflat flanges. This region is pumped by a $1000 \mathrm{~L} / \mathrm{s}$ turbomolecular pump.

For comparison, the MS/MS spectra of the TNT molecular anion, the $\mathrm{m} / \mathrm{z}$ 240 parent ion from PETN, and the m/z 176 parent ion from RDX obtained on the TSQ 700 and those acquired on the ASGDI/QT and ASGDI/ITMS instruments are shown in Figures 41, 42, and 43, respectively. Overall, the spectra are similar to each other but there are qualitative differences due to differences in the mass analyzers and in the collisional activation conditions. For the TSQ 700 and QT, the collisional activation conditions are similar but the analyzers are different ( $Q$ vs. $T$ ). Of particular note is the inferior resolution provided by the QT. The collisional 


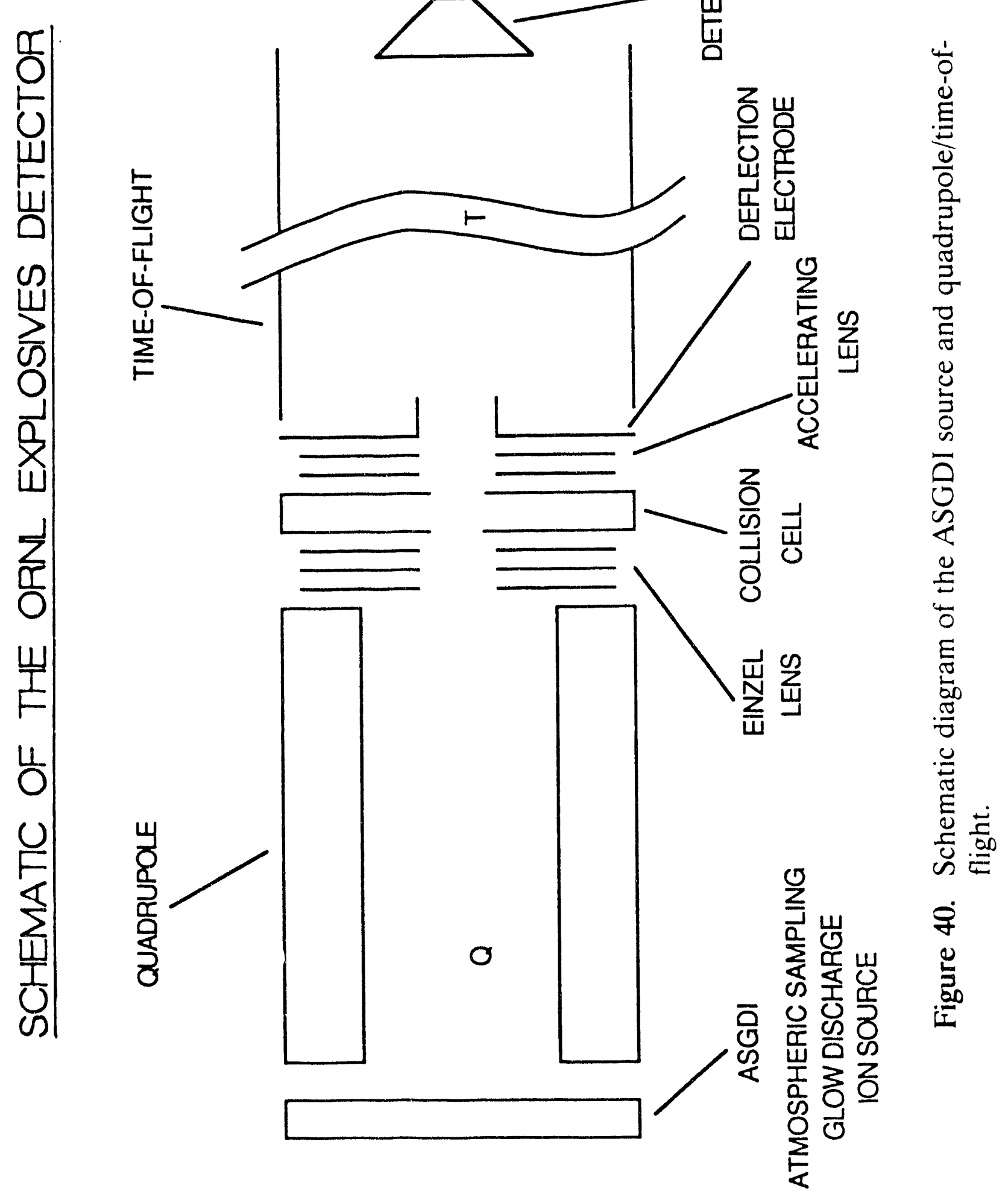


MS/MS of TNT (m/z 227) vs Instrument
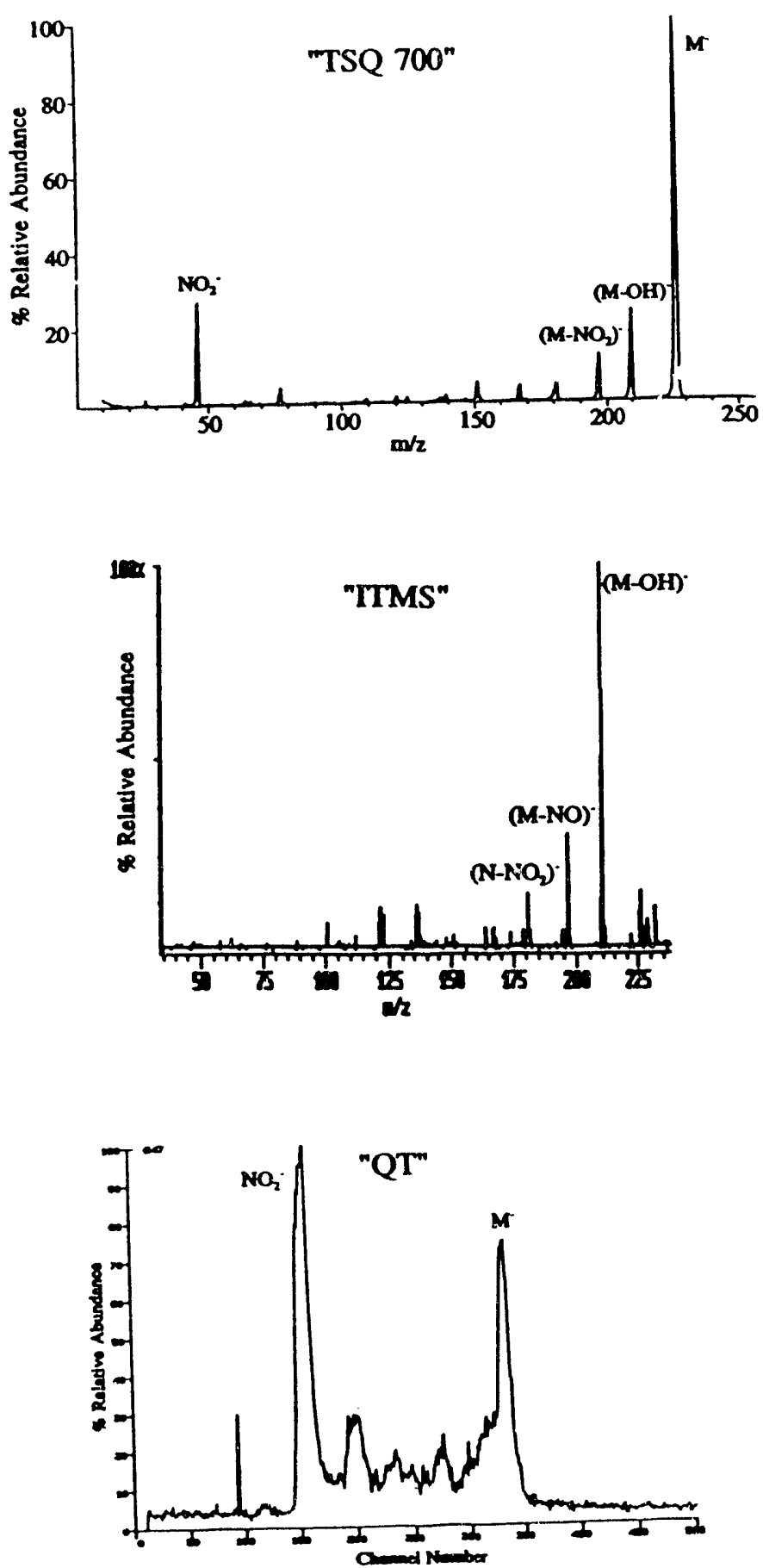

Figure 41. MS/MS of TNT vs. Instrument 
MS/MS of PETN (m/z 240) vs Instrument
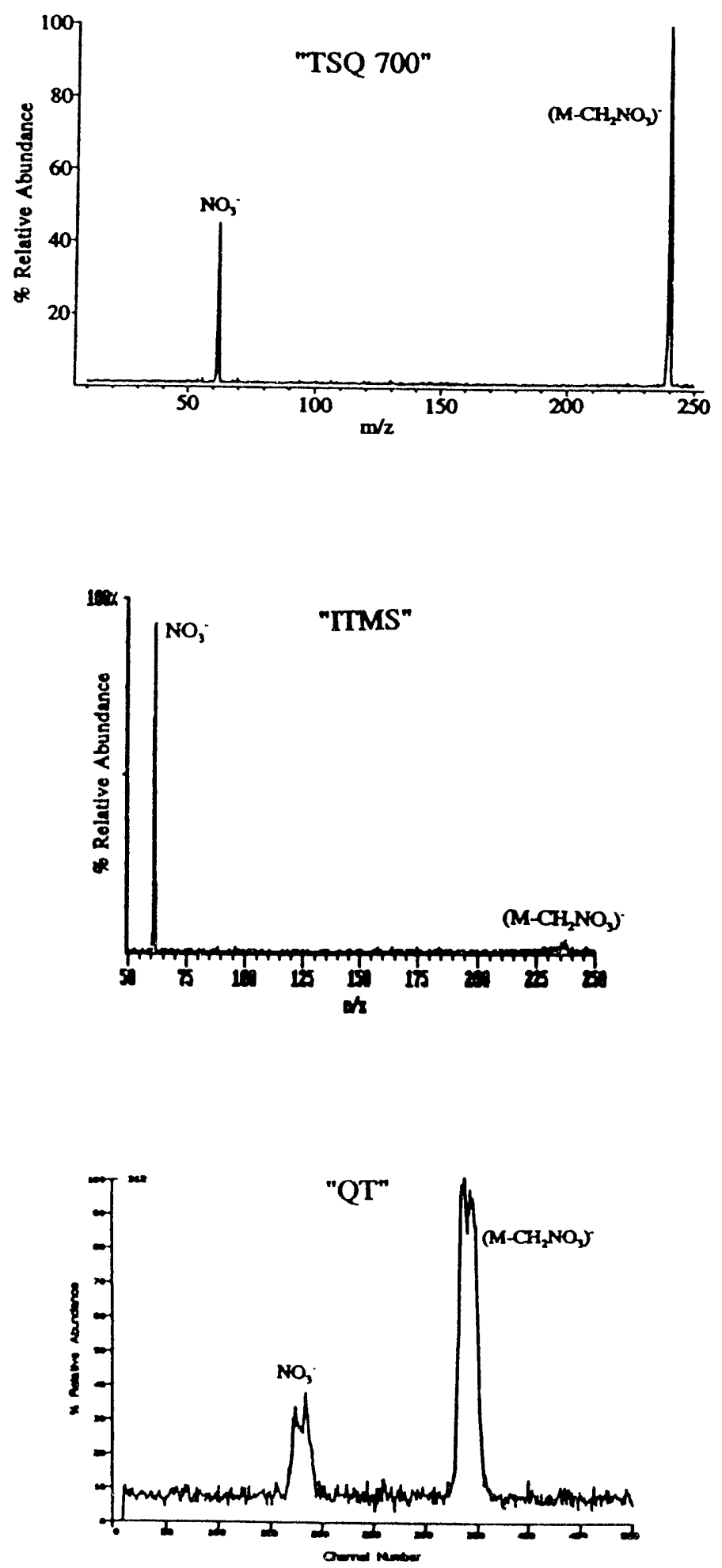

Figure 42. MS/MS of PETN vs. Instrument 
109

MS/MS of $\mathrm{RDX}(\mathrm{m} / \mathrm{z} 176)$ vs Instrument
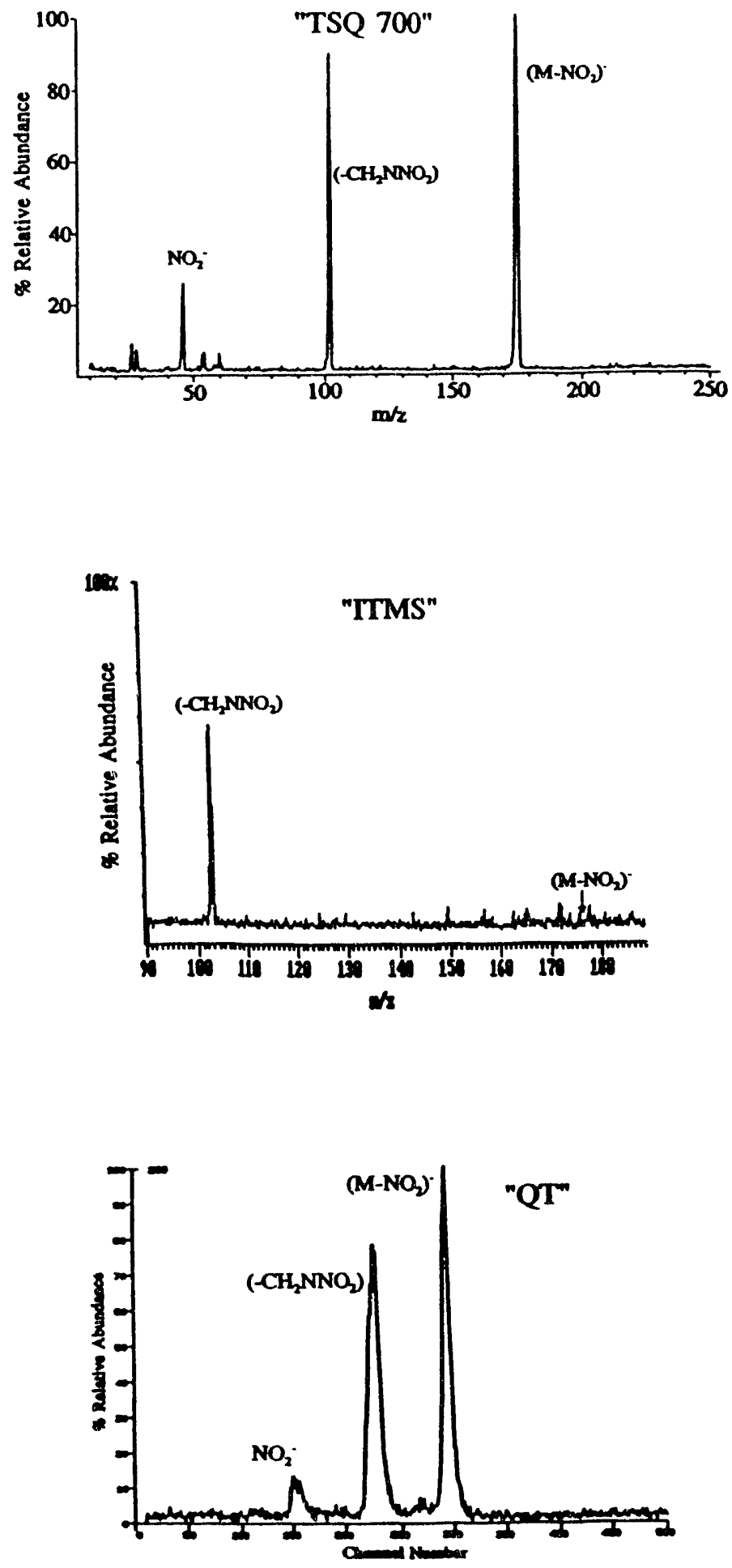

Figure 43. MS/MS of RDX vs. Instrument 
activation conditions in the ion trap differ from those of the TSQ 700 and the QT in that it is a slow heating process which leads to complete dissociation of the parent ion (note the lack of significant parent ion intensity in the MS/MS spectrum). The overall efficiency of the MS/MS process (dissociation and collection) on the ion trap often approaches $100 \%$. The data show similar high mass product ions, but $\mathrm{NO}_{2}$ is not observed in the MS/MS spectrum of TNT on the ion trap because a low mass cut-off of 50 was used to acquire the spectrum.

Since a standard quantifiable vapor generator was not available to us, we sampled the head space vapor of a few hundred milligrams of TNT in a scintillation vial for the direct comparison studies. This provided a reproducible, constant flux of TNT into the various instruments. The data were collected for two seconds to normalize the analyte quantity sampled by each instrument. A quantitative measure of "signal/noise" for each experiment was taken as the analyte signal (less background signal) divided by three times the standard deviation of the background signal. For the beam-type instruments (TSQ and QT), the $\mathrm{NO}_{2}$. product ion was used as the analyte signal whereas the product ion at $\mathrm{m} / \mathrm{z} 210$ was used for the ITMS data. Table IV summarizes the "signal/noise" results acquired under the various MS/MS operating modes. The various operating modes establish compromises between signal/noise and specificity via the normal trade-offs between resolution and transmission, duty cycle, and scan length, etc. These modes were described in Section IV.C relative to the TSQ 700. All of the scan modes are possible with the TSQ 700 while some are either not possible or are difficult to perform with the other geometries. The most direct comparison to evaluate signal/noise at equal specificity comes from the data acquired with Q1 resolution > 400 and $\mathrm{Q} 3$ resolution maximized. For the TSQ 700 and the ITMS, product ion resolving powers are comparable. However, the product ion resolution for the QT is at least an order of magnitude lower. The TSQ 700 provides slightly better signal/background than does the QT with superior specificity. The ITMS, however, provides roughly an order of magnitude greater signal/background with specificity at least as good as that of the TSQ 700 . 
However, if specificity is sacrificed, the beam-type instruments can come closer to the performance of the ITMS. This is apparent in the numbers obtained using various forms of parent ion resolution degradation, including targeted product ion mode. The TSQ 700 can approach the performance of the ITMS in the targeted product ion mode with a narrow scan (to improve duty cycle) over ihe $\mathrm{m} / \mathrm{z} 46$ product ion. An analogous procedure is not available for the QT.

\begin{tabular}{|c|c|c|c|c||}
\hline \multicolumn{5}{||c|}{ Table IV. MS/MS Signal/Noise for Various Instrumental Configurations } \\
\hline \hline TSQ700 OPERATIONAL MODES & \multicolumn{2}{|c|}{ MS/MS Signal/Noise } \\
\hline Q1 & Q3 & TSQ700 & QT & ITMS \\
\hline $\mathrm{m} / \Delta \mathrm{m}>400$ & $\mathrm{~m} / \Delta \mathrm{m}>400$, Full Scan & 30 & - & 200 \\
\hline $\mathrm{m} / \Delta \mathrm{m}>400$ & $\mathrm{~m} / \Delta \mathrm{m} \approx 50$, Full Scan & - & 20 & - \\
\hline RF only, $\mathrm{m} / \mathrm{z}>180$ & $\mathrm{~m} / \Delta \mathrm{m}>400$, Full Scan \\
\hline RF only, $\mathrm{m} / \mathrm{z}>180$ & $\mathrm{~m} / \Delta \mathrm{m} \times 50$, Full Scan & - & - & - \\
\hline RF only, $\mathrm{m} / \mathrm{z}>180$ & $\begin{array}{c}\mathrm{m} / \Delta \mathrm{m}>400, \text { Narrow } \\
\text { Scan }\end{array}$ & 150 & - & - \\
\hline
\end{tabular}

It is clear from this comparison that, as anticipated, the ion trap provides superior sensitivity in MS/MS relative to the beam-type technologies. This follows from the high collection efficiency for product ions and the high fragmentation efficiency afforded by the ion trap collisional activation process. Nevertheless, the TSQ 700, at some sacrifice in specificity, can approach the sensitivity of the ion trap for a single targeted compound. 


\section{INTEGRATION OF THE FAA GLOW DISCHARGE/TRIPLE QUADRUPOLE TANDEM MASS SPECTROMETER WITH THE ION TRACK INSTRUMENTS ROTARY TRAP VAPOR PRECONCENTRATOR}

\section{A. Ion Track Instruments Rotary Trap Vapor Preconcentrator}

Concentration in the rotary trap vapor preconcentrator developed by lon Track Instruments is based on high flow rate air sampling, followed by sample adsorption on a chromatographic stationary phase, and subsequent thermal desorption into a low flow rate effluent stream. A schematic drawing (top view) of the backing plate for the sampling head of the rotary trap is shown in Figure 44 . The surface of the backing plate, fabricated from machineable ceramic, is sealed by silica vapor deposition to ensure that no porcus adsorption sites for explosives vapor are present. A thin channel encompassing the sample air inlet, the detector exhaust, and the main purge inlet is machined between the two concentric ' $O$ '-ring grooves in the top of the backing plate; a cleeper channel is cut between the grooves around the other half of the backing plate. A thin metallic sampling disk (not shown), coated with the chromatographic support, is positioned atop two graphite ' $O$ '-rings seated in the grooves. During operation, the sampling disk rotates in a counterclockwise direction at $5 \mathrm{rpm}$. Two small contact heaters are positioned on top of the sampling disk above the sample air inlet and detector exhaust ports. The heaters are maintained at different temperatures $\left(80^{\circ} \mathrm{C}\right.$ sample inlet, $170{ }^{\circ} \mathrm{C}$ detector exhaust) so that a positive temperature gradient exists between the sample inlet and detector exhaust points.

The sampling disk, channels, and 'O'-rings define the gas path, for sample, purge, and carrier flows in the trap. Moving counterclockwise, the section located between the sample inlet and the first carrier gas inlet is designated as the prestrip region and the section between the two carrier gas inlets as the stripping region. Ambient air is pulled into the system with a multi-stage turbine pump (1 liter-sec ') which pumps the gas path through the vacuum exhaust port. Sample air entering the system $\left(\sim 50 \mathrm{~cm}^{3}-\mathrm{s}^{-1}\right)$ through the sample air inlet impinges upon 


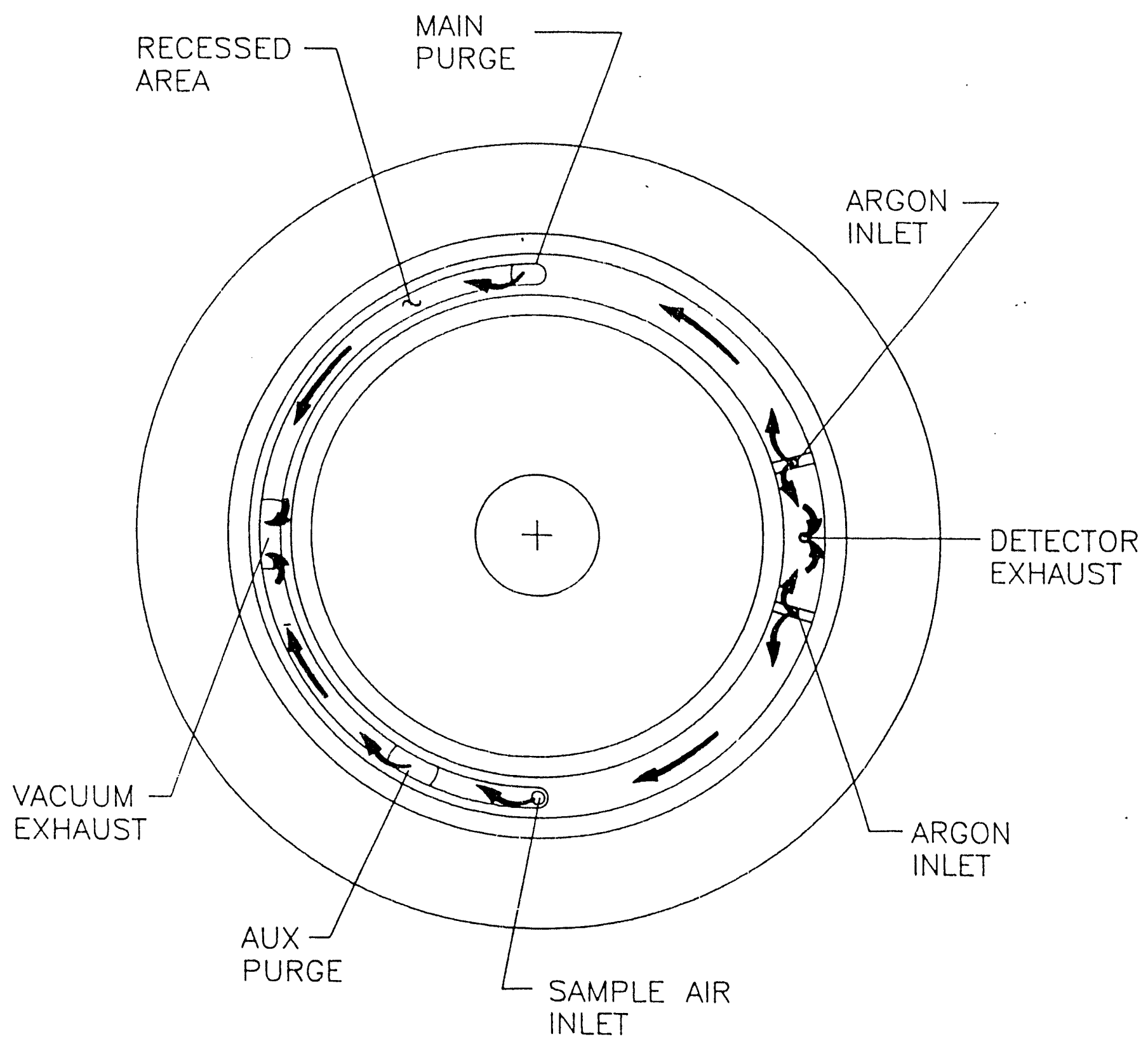

Figure 44. A schematic drawing (top view) of the backing plate for the sampling head of the rotary trap. 
the sampling disk where vapors are trapped by the chromatographic phase. As the sampling disk rotates through the pre-strip region, contaminants are desorbed, entrained in the carrier gas, and carried toward the vacuum exhaust port while explosives compounds remain trapped. As the explosives fraction of the sample enters the stripping region, the temperature is rapidly increased to $170{ }^{\circ} \mathrm{C}$ so that all explosives are desorbed and swept out the detector exhaust by the carrier gas. As the sampling disk continues to rotate past the detector exhaust, it enters the purge region where it is cooled to within a few degrees of ambient temperature by a flow of clean air entering through the main purge port.

\section{B. Performance Characteristics of the Rotary Trap/ASGDI/TSQ700 System}

Before experiments characterizing the rotary trap/ASGDI/TSQ700 system were initiated, a series of experiments were performed with the ASGDI/TSQ700 instrument to determine the effect of the rotary trap on the standard flow rate ( $2.7 \mathrm{~cm}^{3}-\mathrm{s}^{-1}$ ) into the ASGDI source. With only the transfer line attached to the source, the flow rate into the source remained unchanged. When the transfer line was connected between the rotary trap and the ASGDI source, the flow rate at the detector exhaust of the sampling head also was $2.7 \mathrm{~cm}^{3}-\mathrm{s}^{-1}$. Thus, it was concluded that the rotary trap had a negligible effect on the ASGDI sampling rate.

Additional measurements were made to ascertain linearity of the ASGDI/TSQ700 response for explosive vapors. Multiple injections of 1, 2, 3, and $4 \mathrm{~cm}^{3}$ of sample vapor from a heated syringe (supplied by ITI) loaded with RDX were made into the source via the transfer line. A conversion table (also provided by ITI) relating syringe temperature to sample vapor concentration was used to determine the amount of RDX injected. The syringe was heated to $60{ }^{\circ} \mathrm{C}$ corresponding to $11.6 \mathrm{pg}-\mathrm{cm}^{3}$ of RDX. Data for the characteristic RDX signal at $\mathrm{m} / \mathrm{z} 176$ were acquired in single MS mode; the peak area is plotted versus injected volume in Figure 45. The plot confirms the linearity of the ASGDI/TSQ700 response for RDX over a range of about $10 \mathrm{pg}$ to $40 \mathrm{pg}$. The linear dynamic 


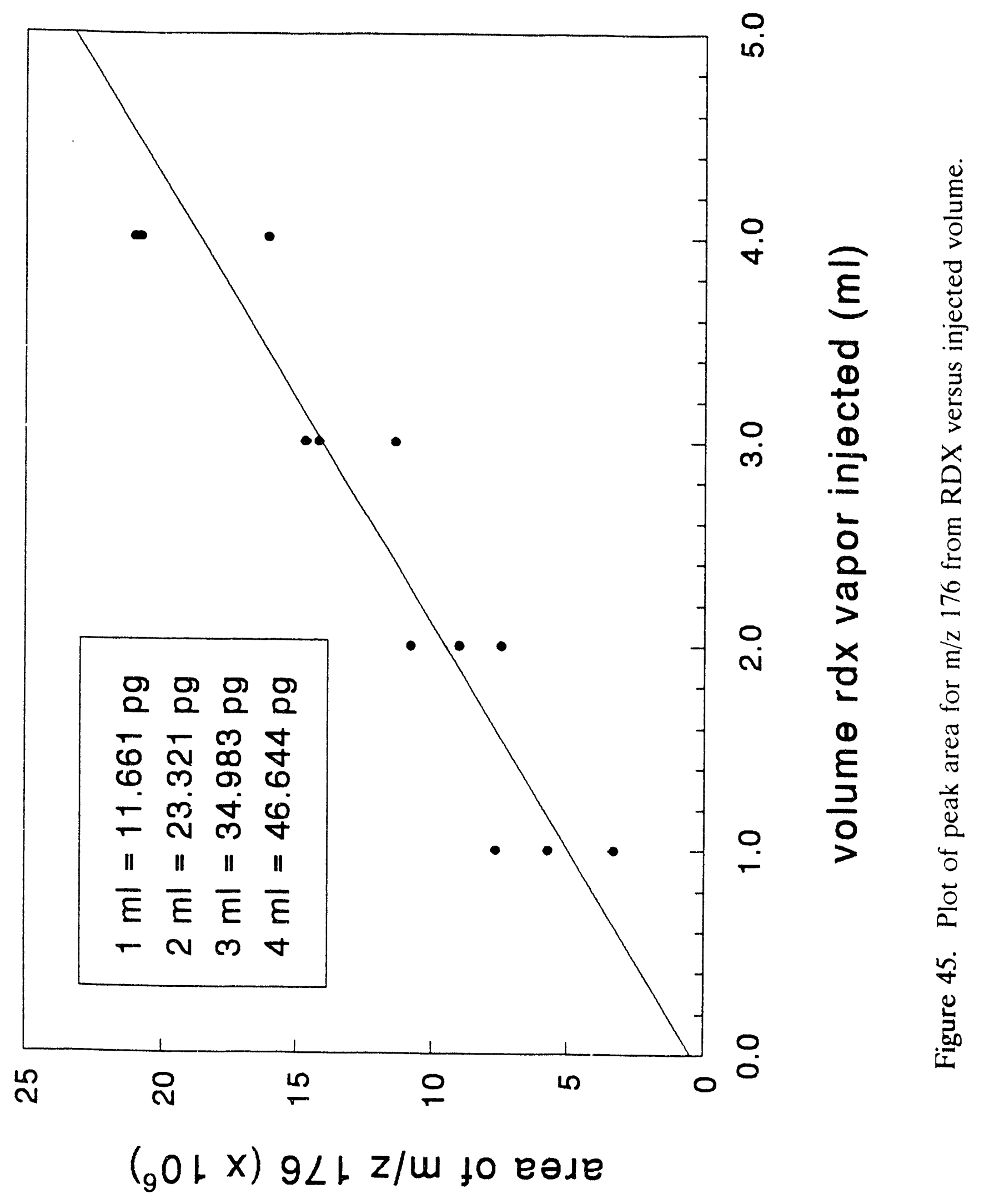


range of the instrument is expected to extended several orders of magnitude beyond this range.

The last step prior to explosives vapor sampling with the rotary trap/ASGDI/TSQ700 system involved optimization of the pre-strip and post-strip carrier gas (zero air) flow rates for the rotary trap. The adjustment was performed by establishing a constant level of TNT vapor and then observing the signal intensity of the TNT molecular anion ( $m / z 227)$ while varying the flow rates. The optimum response was obtained at pre- and post-strip flow rates of $\sim 16 \mathrm{~cm}^{3}-\mathrm{min}^{1}$ as shown in Table $V$. The flowmeter calibration data $\left(\mathrm{cm}^{3}-\mathrm{min}^{-1}\right.$ vs $\mathrm{mm}$ ) were supplied by ITI. All subsequent experiments were performed using these flow rate settings.

The experiments designed to determine the degree of ASGDI/TSQ700 performance enhancement provided by the rotary trap entailed comparison of TNT signal response "without" and "with" the preconcentration unit. The transfer line was always attached to the ASGDI source (although the rotary trap was obviously not connected in the "without" experiments) to eliminate possible ambiguities associated with that parameter. In the firsi set of experiments, a small quantity ( $10 \mathrm{mg}$ ) of crystalline TNT was placed in a closed $20 \mathrm{~mL}$ vial and allowed to equilibrate at ambient temperature. The headspace vapor was then sampled via a short length of Teflon tubing connected to the transfer line only in the "without" experiment, and via the heated sampling probe and rotary trap in the "with" experiment. The mass spectral data (Figure 46), acquired at a rate of one complete mass scan per second, showed similar maximum intensities at $\mathrm{m} / \mathrm{z} 227$ (TNT) for both situations. However, the "without" signal maximum was gradually reached over a period of some 10 minutes, while the "with" signal exhibited a prompt maximum followed by an exponential-type decay to a plateau over roughly the same period. The delayed "without" response was attributed to the time required for TNT molecules to cover the available adsorption sites in the Teflon tubing, while the declining "with" response was presumably due to sample vapor depletion during sampling. 

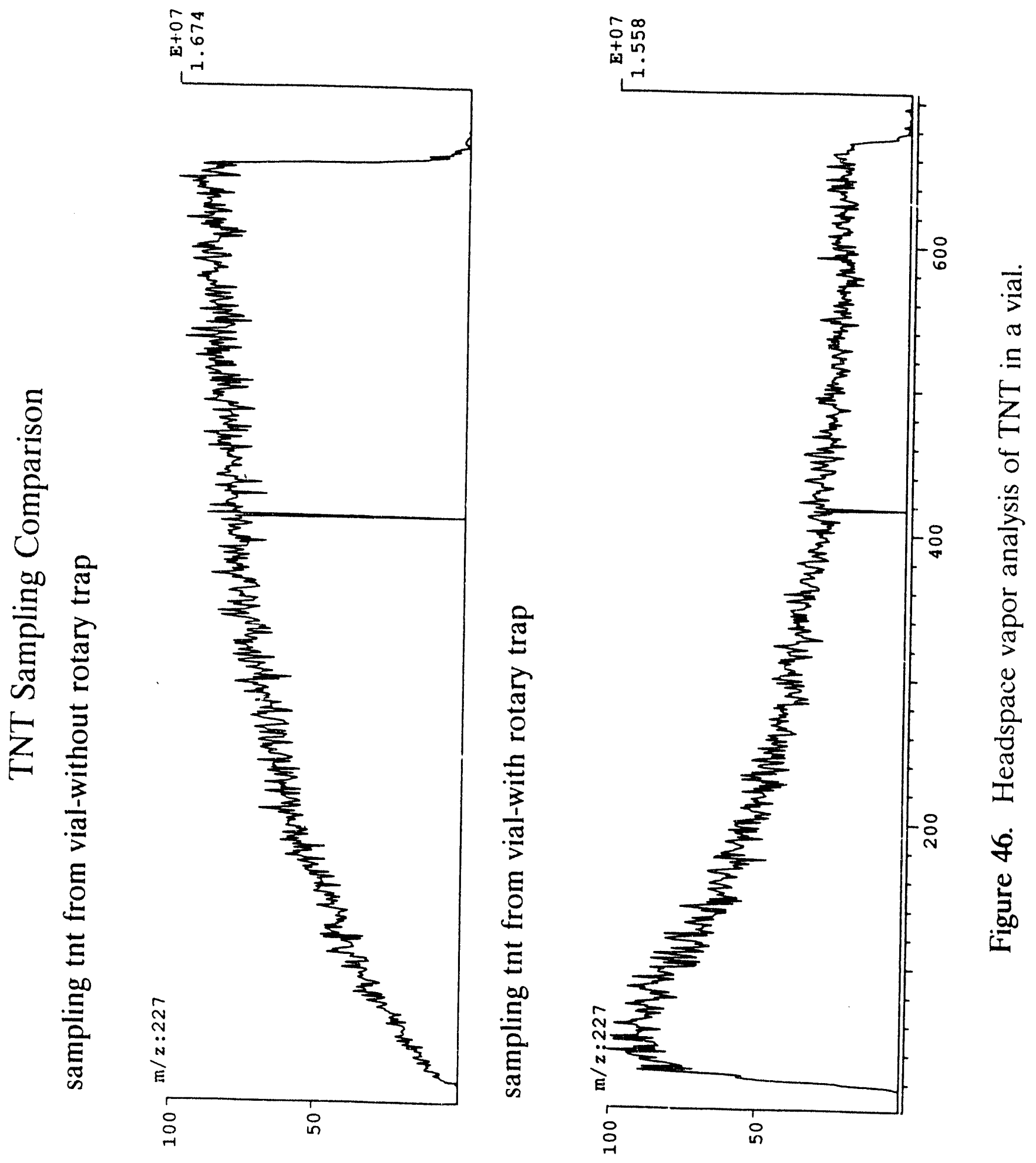


\begin{tabular}{|c|c|c|c|}
\hline $\begin{array}{c}\text { Scan } \\
\text { Numbers }\end{array}$ & $\begin{array}{c}\text { Pre-Strip Flow } \\
\left(\mathrm{cm}^{3}-\mathrm{min}^{1}\right)\end{array}$ & $\begin{array}{c}\text { Post-Strip Flow } \\
\left(\mathrm{cm}^{3}-\mathrm{min}^{3}\right)\end{array}$ & $\begin{array}{c}\text { Avg. Int. } \times 10^{6} \\
@ \mathrm{~m} / \mathrm{z} 227\end{array}$ \\
\hline $154-251$ & 16 & 16 & 2.38 \\
\hline $298-387$ & 25 & 25 & 1.80 \\
\hline $430-530$ & 37 & 37 & 1.32 \\
\hline $582-674$ & 52 & 52 & 1.01 \\
\hline $718-812$ & $<6$ & $<6$ & 1.88 \\
\hline $855-934$ & 7 & 7 & 1.84 \\
\hline $989-1080$ & 16 & 16 & 1.76 \\
\hline $1137-1220$ & 16 & $<6$ & 1.78 \\
\hline $1266-1354$ & 16 & 7 & 1.68 \\
\hline $1399-1486$ & 16 & 25 & 1.36 \\
\hline $1517-1611$ & 16 & 37 & 1.12 \\
\hline 1645-??7? & $<6$ & 16 & 2.04 \\
\hline $1774-1867$ & 7 & 16 & 1.64 \\
\hline $\begin{array}{l}1920-1962 \\
1966-1996 \\
\end{array}$ & 25 & 16 & 1.56 \\
\hline $2048-2128$ & 37 & 16 & 1.34 \\
\hline $2171-2253$ & 7 & $<6$ & 1.59 \\
\hline $2298-2386$ & 7 & 25 & 1.30 \\
\hline $2431-2520$ & 7 & 37 & 1.23 \\
\hline $2565-2654$ & $<6$ & 7 & 1.56 \\
\hline $2694-2785$ & 25 & 7 & 1.47 \\
\hline $2813-2912$ & 37 & 7 & 1.35 \\
\hline
\end{tabular}

The experiments weie then modified to avoid the sampling problems noted above. The open sample vial was placed in a large $(6 \times 6 \times 6$ inches), heated ( 40 C) box, and a heated stainless steel tube was inserted through the box wall 
so that the vapors could be sampled with either the transfer line (sans Teflon tubing) or the sampling probe. Experimental data (Figure 47) obtained using this apparatus now showed no variation in signal over the sampling period ( $10 \mathrm{~min}$.), but there was still no discernible signal enhancement with the rotary trap. Triplicate $1 \mathrm{~min}$. "without" and "with" sampling experiments were also performed with the same apparatus. The data, shown in Figure 48, also indicate no evidence $c^{f}$ signal enhancement with the rotary trap.

Given the inlet flow rates of about $50 \mathrm{~cm}^{3}-\mathrm{s}^{-1}$ for the preconcentrator and 2.7 $\mathrm{cm}^{3}-\mathrm{s}^{1}$ for the ASGDI/TSQ 700, the maximum possible concentration enhancement, as determined by the ratio of the inlet flow rates, is slightly less than twenty. This assumes that there are no losses along the way in material transport from the preconcentrator inlet to the mass spectrometer inlet. The fact that no apparent enhancement was observed might suggest a transport efficiency of roughly $5 \%$. There are two obvious ways, therefore, to observe a concentration effect. One is to improve transport efficiency by identifying the major loss points and addressing them, if possible. Another is to simply reduce the inlet flow rate of the TSQ 700 to improve the inlet ratio. In our preliminary studies, we could not clearly identify any easily correctable problems in material transport. Nevertheless, there is obviously room for improvement here and it might be reasonable to expect to achieve an enhancement of a factor of five or so, based on the efficiency of the preconcentrator coupled with an electron capture detector. The flow rate into the TS2 700 is not easily adjustable without changing the source pressure. We therefore could not vary the inlet flow rate ratio by adjusting the mass spectrometer sampling rate. However, our preliminary work with smaller sampling rate ASGDI with the ion trap system show excellent prospects for reducing the inlet flow rate of the mass spectrometer without adversely affecting sensitivity. For the reasons just given, despite the preliminary studies indicating little concentration effect, the rotary trap concept for explosives vapor concentration remains of interest for explosives detection with tandem mass spectrometry. 

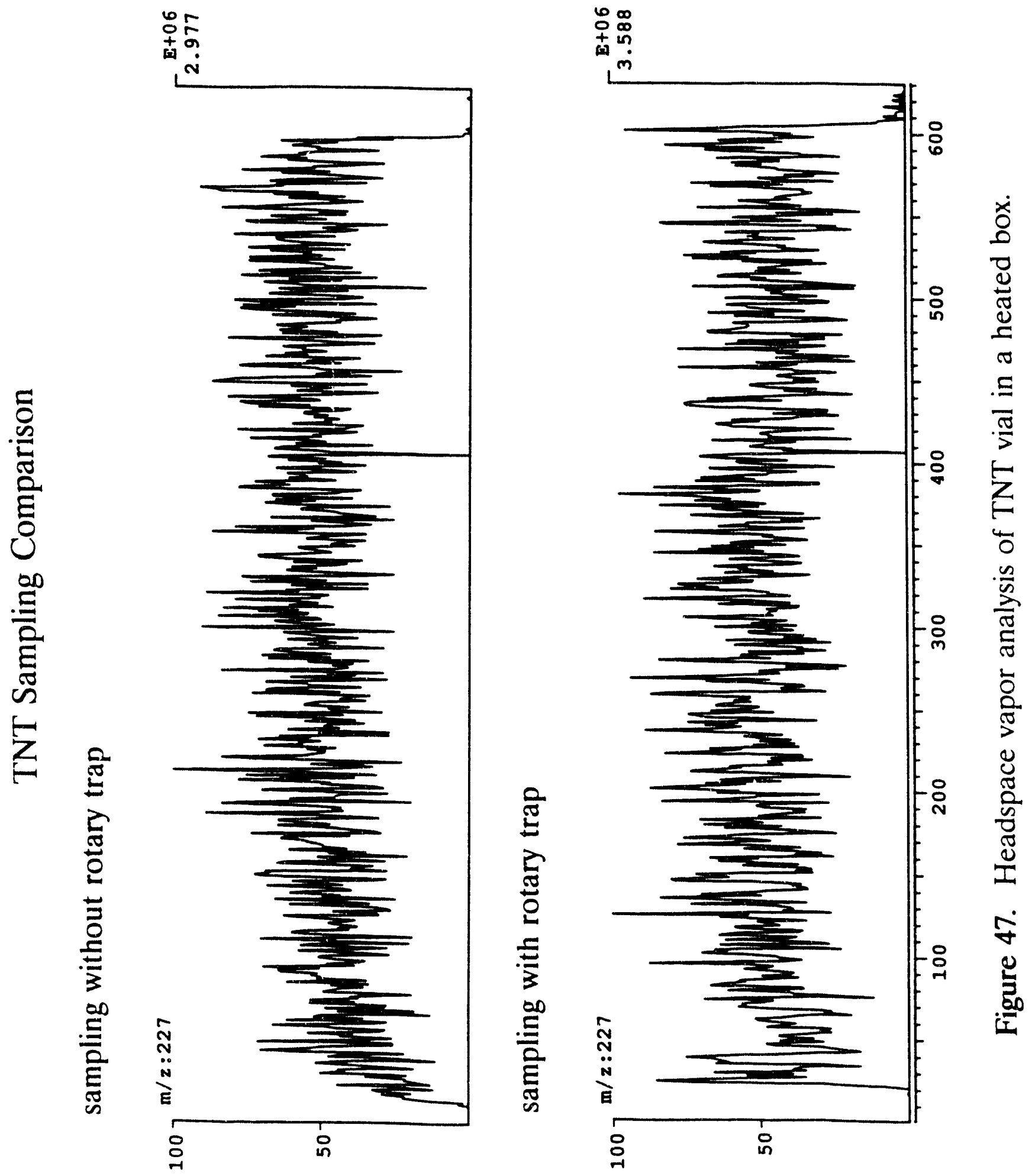

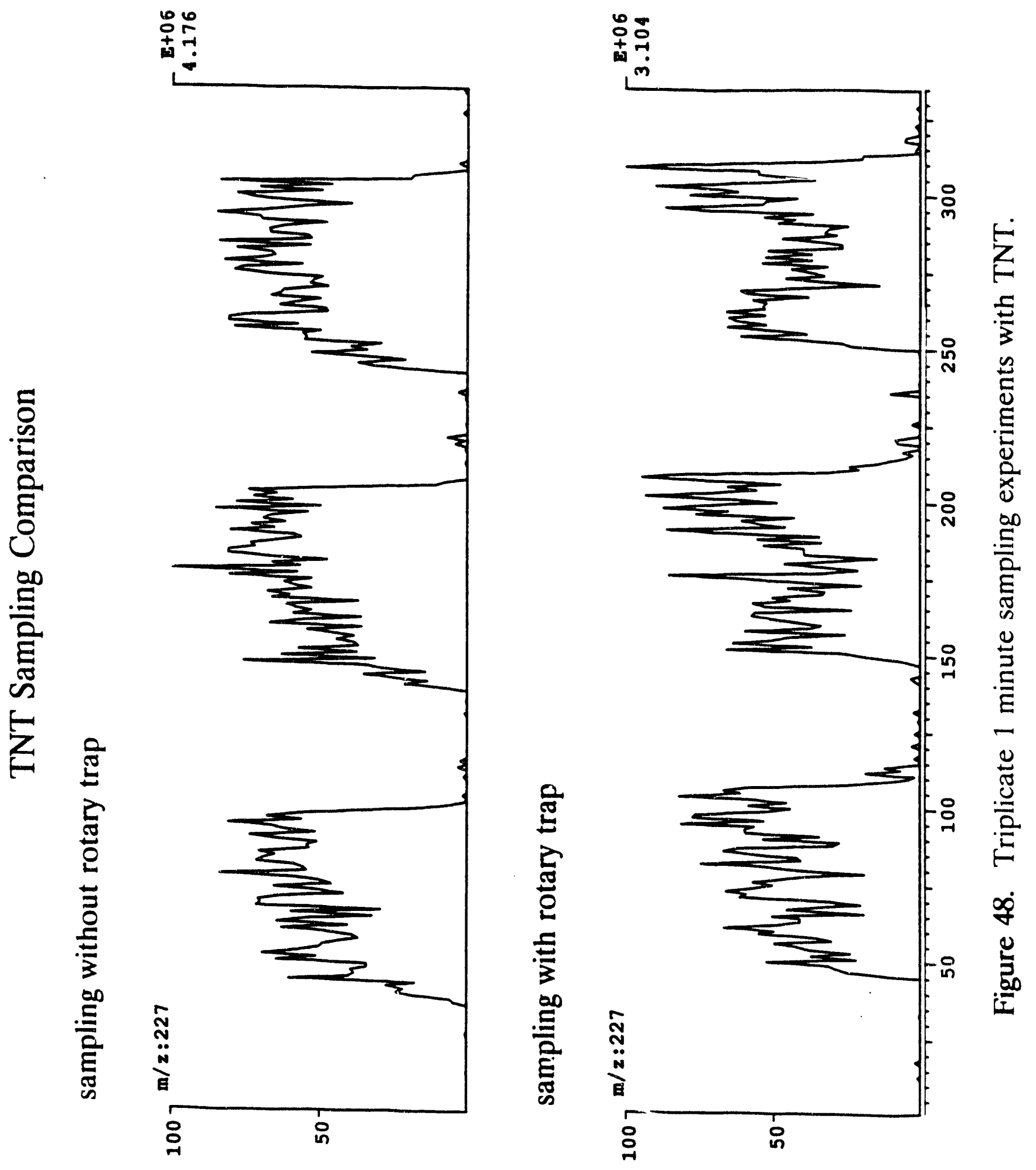


\section{SUMMARY AND PROGNOSIS}

The atmospheric sampling glow discharge ionization/triple quadrupole tandem mass spectrometer is a powerful analytical tool for the development of explosives vapor detection equipment. In particular, its strengths are low to subpicogram detection limits with unusually high specificity. Although not emphasized in this report, the system can also be used to identify unknown species and can be readily adapted for the identification of a wide variety of targeted compounds. In other words, the system can adapt to new threats as they evolve. This might entail the identification of diagnostic parent ions and product ions in the ASGDI/MS/MS procedure and straightforward software changes to modify the automated detection system. If the targeted species are much different chemically from high explosives, significant modification to the ionization method might be required. Nevertheless, there are very few species of moderate size that cannot be converted to gaseous ions with at least one of the wide variety of ionization methods developed for organic mass spectrometry.

Given the specificity and sensitivity of the system, it can serve as a reference analytical device for evaluating the performance of less specific systems and can be used to evaluate the performance of various approaches to sampling and preconcentration. It can also be used to evaluate the purity of explosives when operated with the gas chromatograph supplied with the system. However, despite the fact that the system has been automated for explosives detection, the full potential of the system as an analytical tool for explosives detector development requires a well-trained and highly skilled operator. A field-deployable explosives detector based on beam-type tandem mass spectrometry technology need not be as powerful as the TSQ700. The ASGDI/TSQ700 is capable of a wide variety of experiments, many of which would certainly be of use in support of explosives detection research and development. The operator should therefore be aware of these experiments and what information they can provide and must 
be able to implement them on a system that is almost totally under software control.

In terms of field-deployed explosives vapor detection with tandem mass spectrometry, the triple quadrupole geometry would appear to be the most appropriate beam-type tandem mass spectrometer. Indeed, a triple quadrupole system for this purpose is already commercially available, although it employs atmospheric pressure ionization rather than ASGDI. The cost and size of this equipment has been a barrier to its more widespread use. The rapid development of the quadrupole ion trap as an analytical tandem mass spectrometer over the past decade has seen this technology come to rival and now surpass the more mature beam-type technologies for many targeted compound applications. In terms of size, cost, and sensitivity, the ion trap is clearly more attractive as a field device. Until very recently, the finite storage capacity of the device was its greatest limitation in real-world applications. However, these limitations have been largely overcome with mass-selective ion accumulation and universal collisional activation techniques. For this reason, it is recommended that the ASGDI/TSQ700 system be regarded as a laboratory-based tool which can be of significant value in support of an explosives detection research and development program. For explosives vapor detection technology for the field, the quadrupole ion trap coupled with an atmospheric sampling ionization method appears to be the most promising route to making a cost-effective system with the specificity of tandem mass spectrometry available for protection against terrorism. At ORNL, atmospheric sampling glow discharge ionization has proved to be a fast, rugged, sensitive, and inexpensive means for ionizing explosives vapors present in air with minimal matrix effects. 


\section{$X$. REFERENCES}

1. J. Yinon and $S$. Zitrin, "The Analysis of Explosives", Pergamon Series in Analytical Chernistry, Volume 3, Pergamon Press, Oxford, 1981.

2. J. Yinon Mass Spectrom. Rev., 1 (1982) 257.

3. S.A. McLuckey, G.L. Glish, and J.A. Carter J. Forens. Sci., 30 (1985) 773.

4. S.N. Ketkar, J.G. Dulak, W.L. Fite, J.D. Buchner, and S. Dheandhanoo Anal. Chem. 61 (1989) 260.

5. B.A. Thomson, W.R. Davidson, and A.M. Lovett, Environ. Health Perspectives, 36 (1980) 77.

6. J.B. French, B.A. Thomson, W.R. Davidson, N.M. Reid, J.A. Buckley, "Mass Spectrometry in the Environmental Sciences", F.W. Karasek (Ed.), Pergamon Press, Oxford, 1982.

7. S. Boumsellek and A. Chutjian Anal. Chem., 64, (1992) 2096.

8. S. Boumsellek, S.H. Alajajian, and A. Chutjian J.Am. Soc. Mass Spectrom., 3. (1992) 243.

9. S.A. McLuckey, G.L. Glish, K.G. Asano, and B.C. Grant Anal. Chem., $\underline{60}$ (1988) 2220.

10. G.E. Spangler and M.J. Cohen in "Plasma Chromatography", T.W. Carr (Ed.), Plenum Publishing Corp., New York, 1984.

11. E.C. Horning, M.G. Horning, D.I. Carroll, I. Dzidic, and R.N. Stillwell Anal. Chem. 45, (1973) 936.

12. M. Mckeown and M.W. Siegal, Am. Lab. November, 89 (1975).

13. J.B. French, N.M. Reid and J.A. Buckley, U.S. Patent 4,121,099, Oct. 17 1978.

14. H. Kambara and I. Kanomata, Mass Spectrosc., 24. (1976) 229.

15. H. Kambara and I. Kanomata, Mass Spectrosc., 24, (1976) 271. 
16. J.R. Hobbs, U.S. D.O.T. Report No. FAA-RD-74-134, Transportation Systems Center, Cambridge, MA (1974).

17. D.M. Chambers, S.A. McLuckey, and G.L. Glish Anal. Chem., 65 (1993) 778.

18. P.J. Slevin and W.W. Harrison Appl. Spectrosc. Rev., 10 (1975) 201.

19. J.M. Hayes Chem. Rev., 87 (1987) 745.

20. R. Campargue "Rarefied Gas Dynamics", Vol. 2, J.H. deLeeuw (Ed.), Academic Press, New York, (1966), p. 279.

21. D.M. Lubman, C.T. Rettner, R.N. Zare, J. Phys. Chem., 86 (1982) 1129.

22. K. Bier and B. Schmidt Z. Angew. Phys., 11 (1961) 34.

23. K.B. Mitchell J. Opt. Soc. Am., $\underline{51}$ (1961) 846.

24. S.G. Lias, J.E. Bartmess, J.F. Liebman, J.L. Holmes, R.D. Levin, and W.G. Mallard, J. Phys. Chem. Ref. Data, Supplement No. 1, 17 (1988).

25. R.A. Flurer, G.L. Glish, and S.A. McLuckey, J. Am. Soc. Mass Spectrom., 1 (1990) 217.

26. H.S.W. Massey "Negative lons", Cambridge: New York, 1976, Ch. 8.

27. A.G. Harrison "Chemical Ionization Mass Spectrometry", CRC Press, Boca Raton, FL, 1983.

28. K.L. Busch, G.L. Glish, and S.A. McLuckey, "Mass Spectrometry/Mass Spectrometry: Techniques and Applications of Tandem Mass Spectrometry", VCH Publishers, New York, 1988.

29. "Tandem Mass Spectrometry", F.W. McLafferty (Ed.), Wiley and Sons, New York, 1982.

30. S.A. McLuckey J.Am. Soc. Mass Spectrom., $\underline{3}$ (1992) 599.

31. D.D. Fetterolf and R.A. Yost, Int. J. Mass Spectrom. Ion Proc., 62 (1984) 33.

32. R.A. Yost and C.G. Enke J. Am. Chem. Soc., 100 (1978) 2274.

33. R.A. Yost and C.G. Enke Anal. Chem., 51 (1979) 1251 A. 
34. G.L. Glish and S.A. McLuckey Anal. Instrum., 15 (1986) 1.

35. J.D. Ciupek, J.W. Amy, R.G. Cooks, and A.E. Schoen Int. J. Mass Spectrom. Ion Proc., 6 5, (1985) 141.

36. G.L. Glish and S.A. McLuckey Org. Mass Spectrom. 24 (1989) 470.

37. S.A. Mcluckey, G.L. Glish, and B.C. Grant Anal. Chem., $\underline{62}$ (1990) 56.

38. T.A. Lehman and M.M. Bursey "Ion Cyclotron Resonance Spectrometry", John Wiley and Sons, New York, 1976.

39. R.E. March and R.J. Hughes "Quadrupole Storage Mass Spectrometry", John Wiley and Sons, New York, 1989.

40. M.B. Comisarow and A.G. Marshall Chem. Phys. Lett., $\underline{25}$ (1974) 282.

41. B.S. Freiser in "Techniques for the Study of Ion-Molecule Reactions", J.M. Farrar and W.H. Saunders, Jr. (Eds.), John Wiley and Sons, New York, 1988.

42. R.B. Cody, J.A. Kinsinger, S. Ghaderi, I, J, Amster, F.W. McLafferty, and C.G. Brown Anal. Chim. Acta, 178 (1985) 43.

43. C.L. Wilkins, A.K. Chowdhury, L.M. Nuwaysir, and M.L. Coates Mass Spectrom. Rev., 8 (1989) 67.

44. A.G. Marshall and P.B. Grosshans Anal. Chem., 63 (1991) 215A.

45. W. Paul and H. Steinwedel, U.S. Patent 2,939,952, June, 7, 1960.

46. W. Paul Angew. Chem. Int. Ed. Engl., 29 (1990) 739.

47. J.F.J. Todd Mass Spectrom. Rev., 10 (1991) 3.

48. J.V. Johnson, R.A. Yost, P.E. Kelley and D.C. Bradford Anal. Chem., 62 (1990) 2162.

49. R.E. March Int. J. Mass Spectrom. Ion Proc., 118/119 (1992) 71.

50. R.G. Cooks and R.E. Kaiser, Jr. Acc. Chem. Res., 23 (1990) 213. 
51. R.G. Cooks, G.L. Glish, S.A. McLuckey, and R.E. Kaiser, Jr. Chem. Eng. News, $\underline{69}$ (1991) 26.

52. G.C. Stafford, Jr., P.E. Kelley, and D.R. Stephens, U.S. Patent 4,540,884 (1985).

53. G.C. Stafford, Jr., P.E. Kelley, J.E.P. Syka, W.E. Reynolds, and J.F.J. Todd Int. J. Mass Spectrom. Ion Phys., 60 (1984) 85.

54. S.A. McLuckey, D.E. Goeringer, and G.L. Glish J. Am. Soc. Mass Spectrom., 2 (1991) 11 .

55. J.N. Louris, R.G. Cooks, J.E.P. Syka, P.E. Kelley, G.C. Stafford, Jr., J.F.J. Todd Anal. Chem., 59 (1987) 1677.

56. S.A. McLuckey, G.L. Glish, and P.E. Kelley Anal. Chem., $\underline{59}$ (1987) 1670.

57. S.A. McLuckey, D.E. Goeringer, and G.L. Glish Anal. Chem., 64 (1992) 1455

58. J.N. Louris, J.S. Brodbelt-Lustig, R.G. Cooks, G.L. Glish, G.J. Van Berkel, and S.A. McLuckey Int. J. Mass Spectrom. Ion Proc., 96 (1990) 117.

59. S.A. McLuckey, G.L. Glish, and G.J. Van Berkel Int. J. Mass Spectrom. Ion Proc., 106 (1991) 213.

60. S.A. McLuckey, G.L. Glish, and K.G. Asano Anal. Chim. Acta, 225 (1989) 25.

61. B.A. Eckenrode, G.L. Glish, and S.A. McLuckey Int. J. Mass Spectrom lon Proc., 99 (1990) 151.

62. B.C. Grant, S.A. McLuckey, and G.L. Glish "Proceedings of the 38th ASMS Conference on Mass Spectrometry and Allied Topics", Tuscon, AZ, June 38, 1990, p. 195.

63. D.H. Williams and I. Howe "Principles of Organic Mass Spectrometry", McGraw-Hill, London, 1972, p. 126.

64. S.A. McLuckey and G.L. Glish Org. Mass Spectrom., 22 (1987) 224.

65. S.A. McLuckey and G.L. Glish Int. J. Mass Spectrom. Ion Proc., 76 (1987) 41. 
66. F.J. Conrad, D.W. Hannum, B.C. Grant, S.A. McLuckey, and H.S. McKown "Proceedings of the Third Symposium on Analysis and Detection of Explosives", Mannheim-Neuostheim, FRG, July 10-13, 1989, p.35-1.

67. G.L. Glish, S.A. McLuckey, and H.S. McKown Analytical Instrumentation, 16 (1987) 191. 
XI. APPENDIX I

Machine drawings for the ASGDI source for the TSQ700 


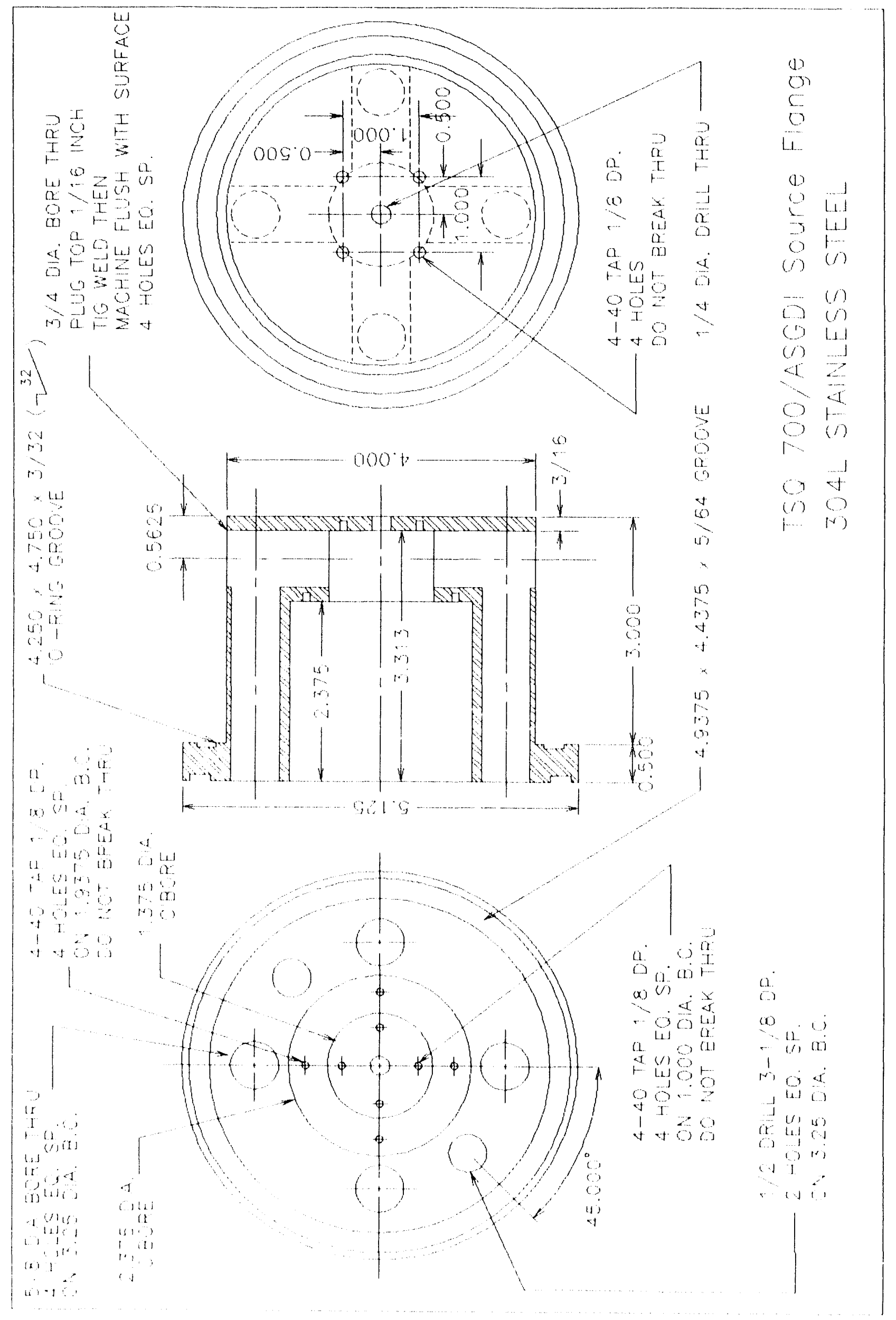



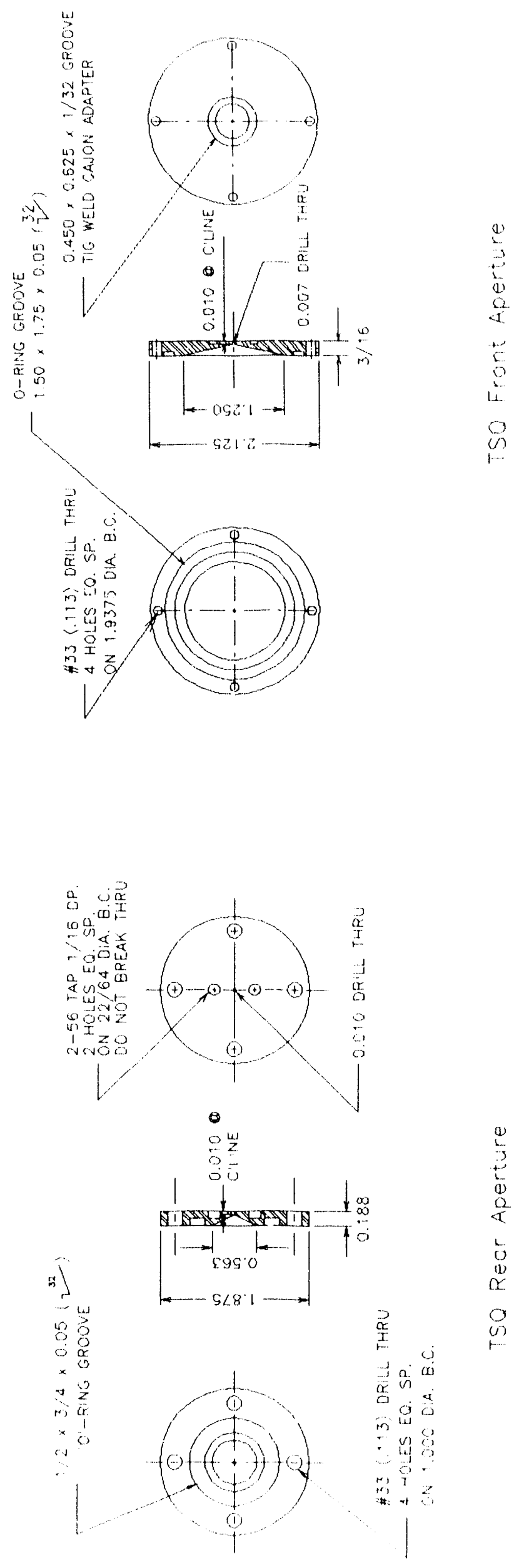

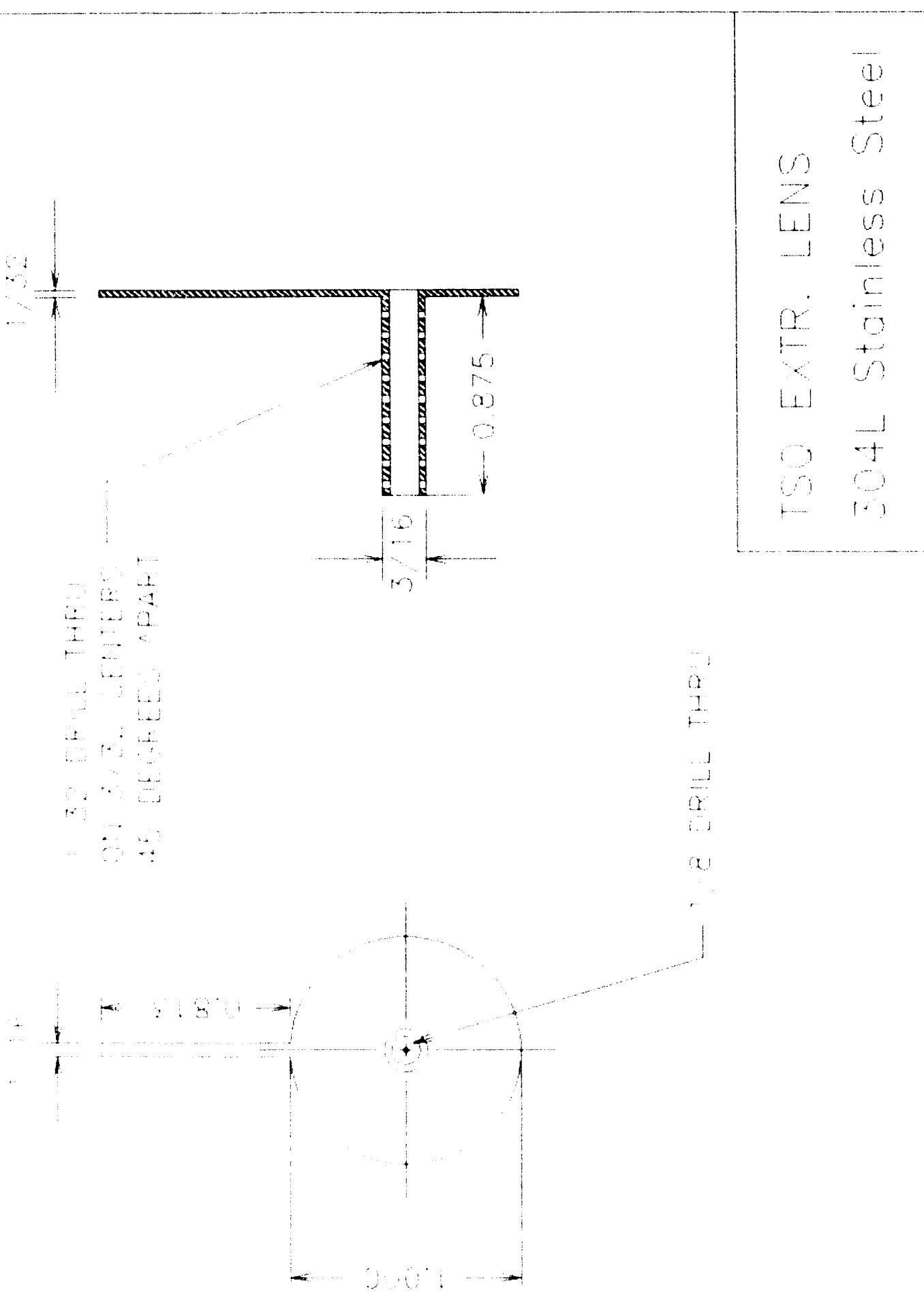
XII. APPENDIX II

ICL procedures for automated explosives detection. 


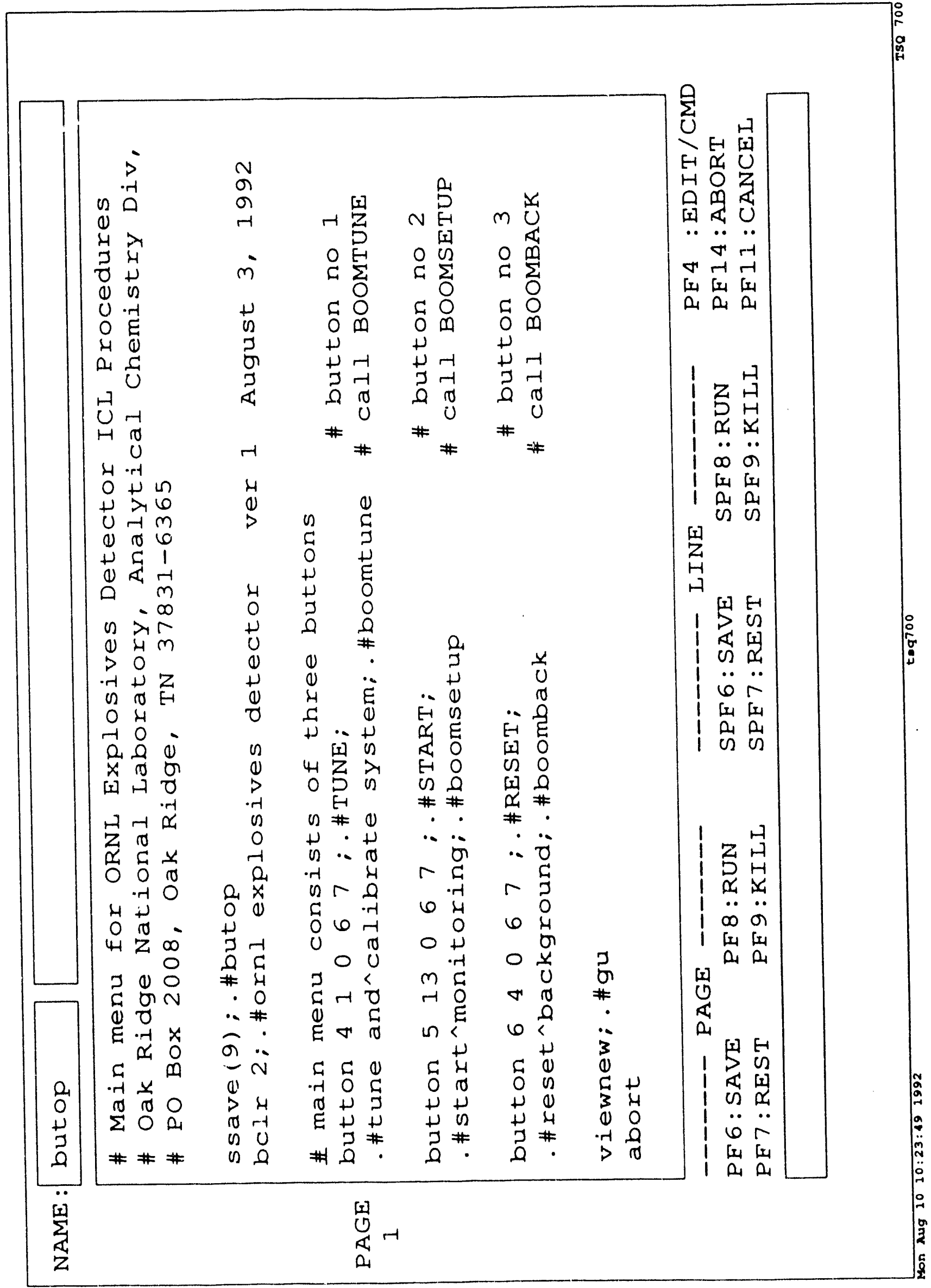




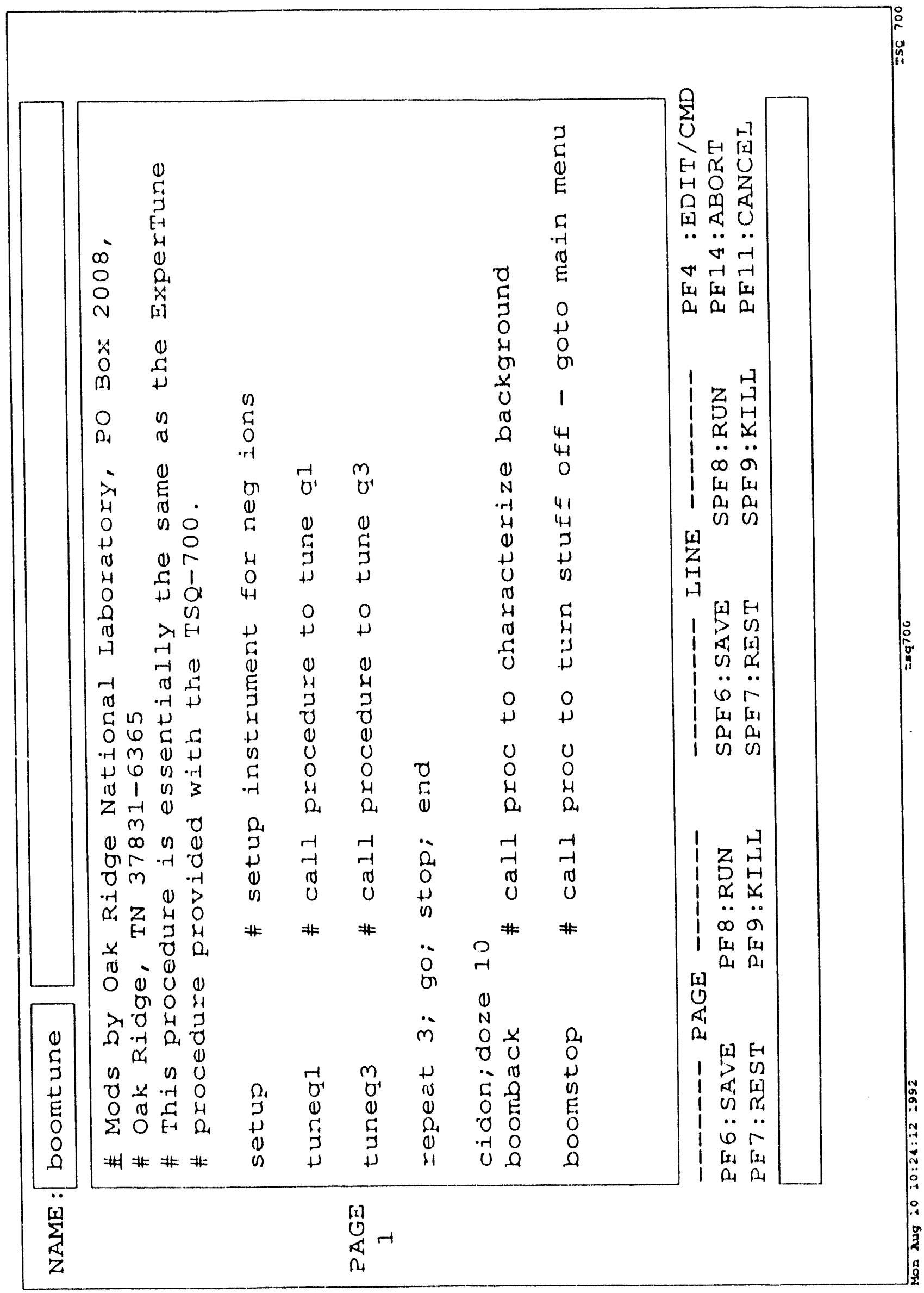




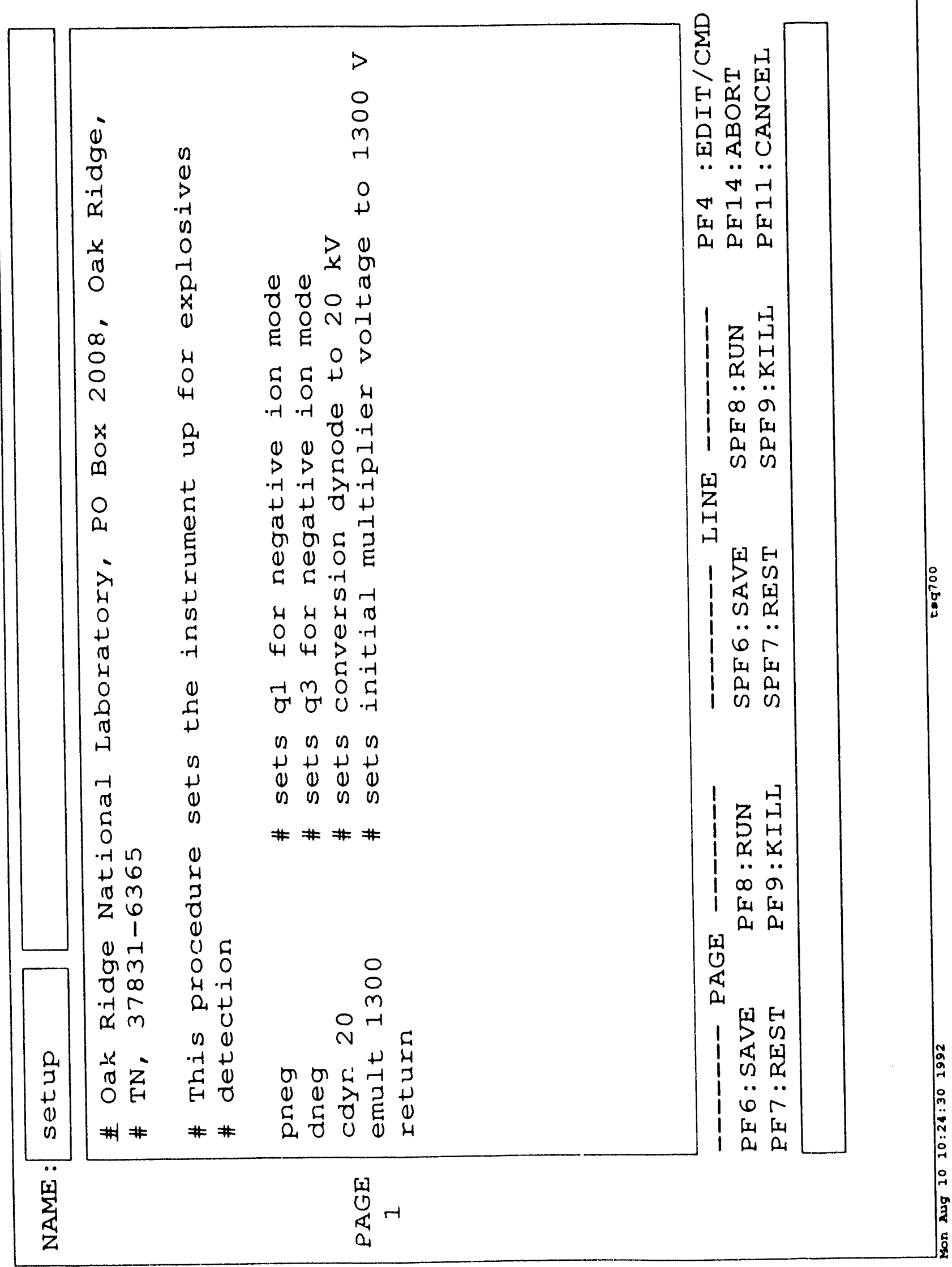




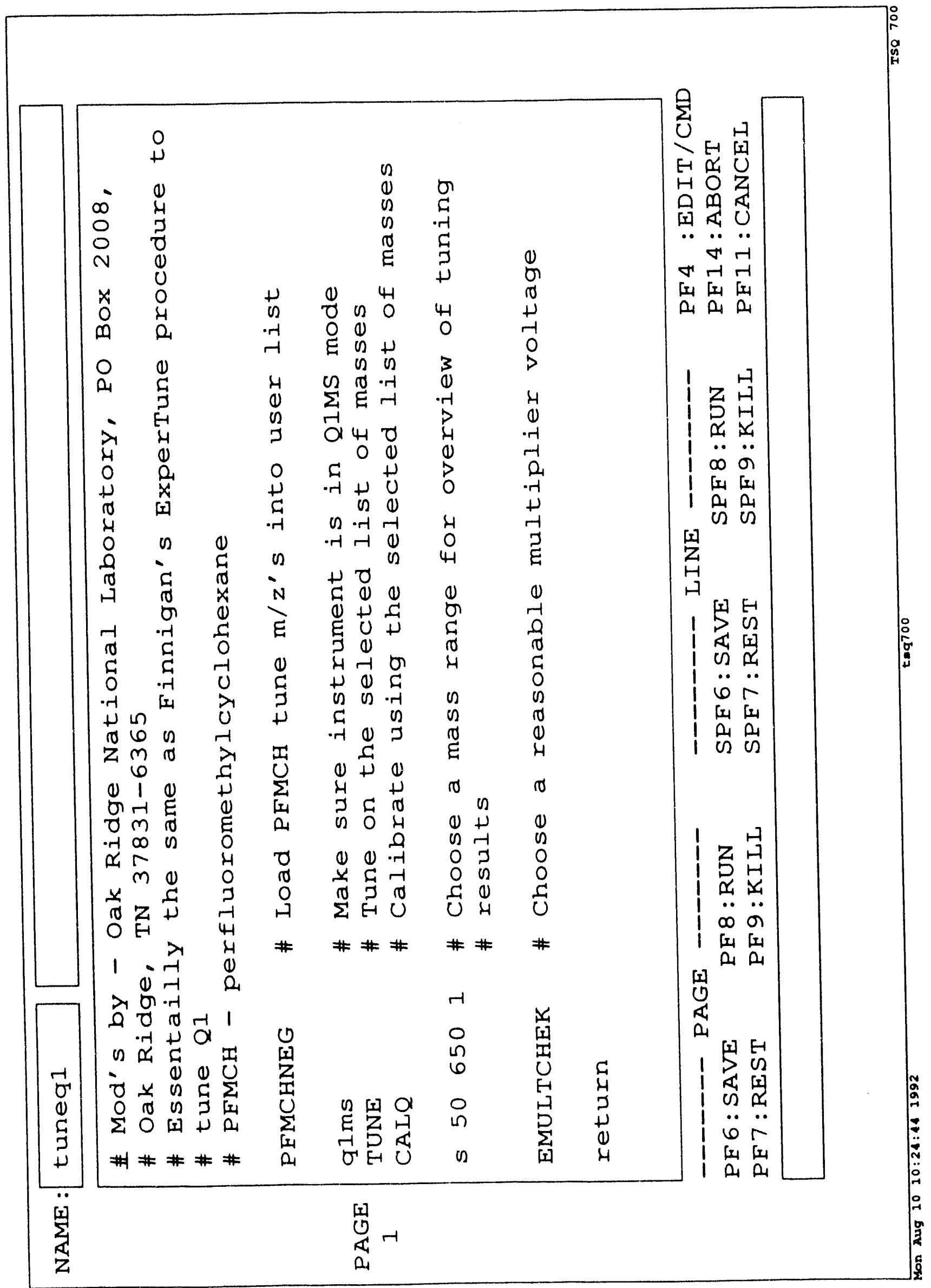




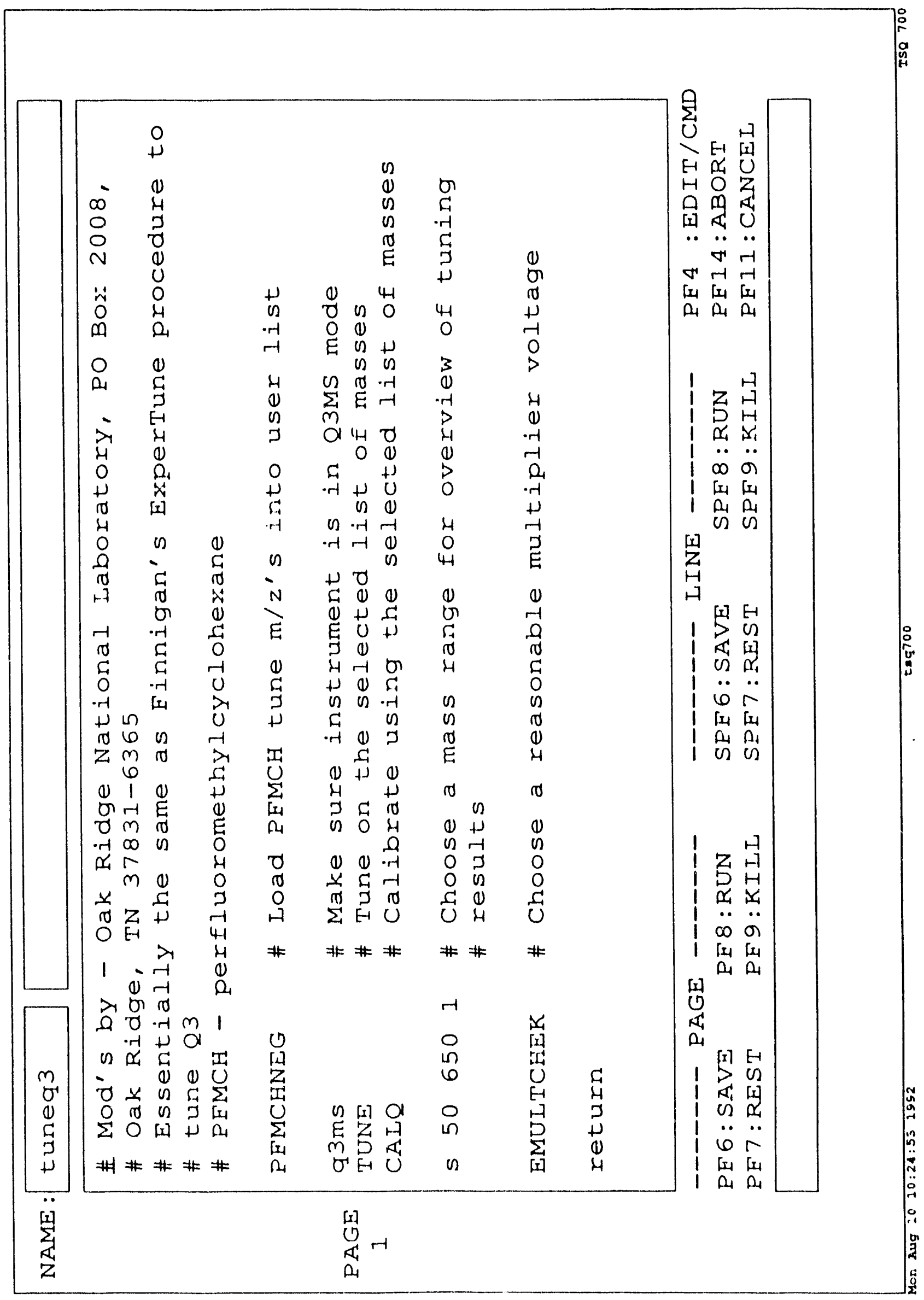




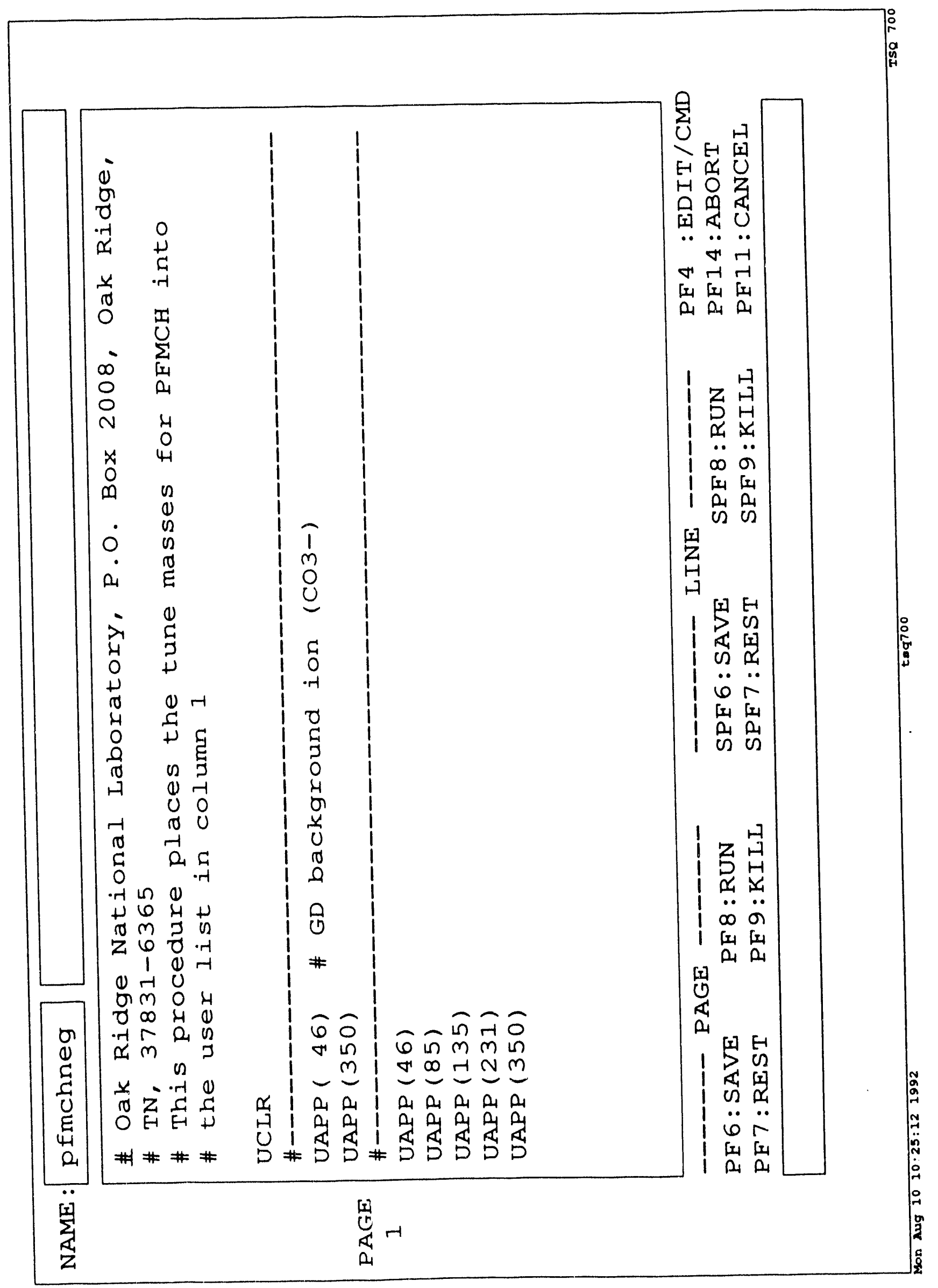




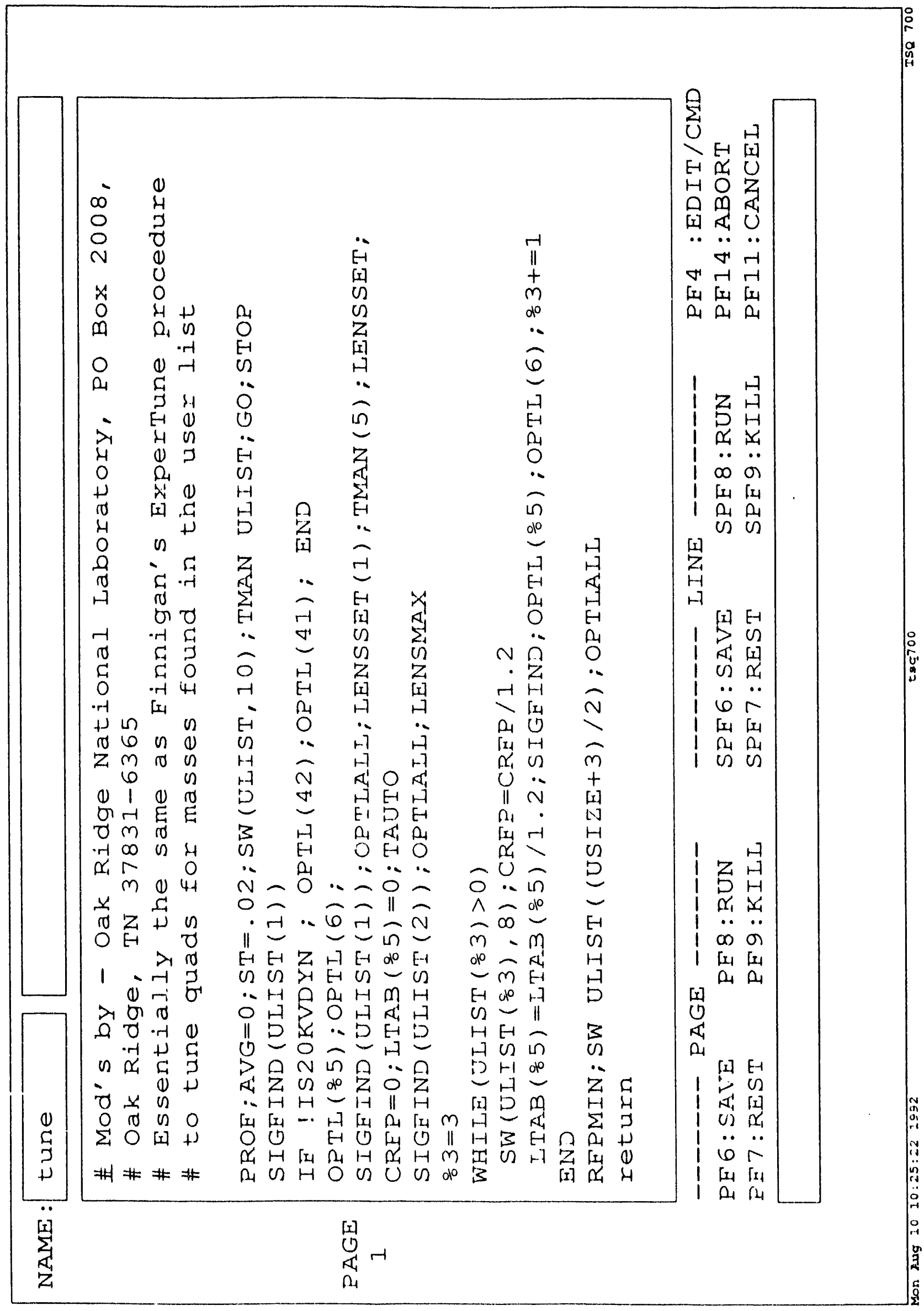




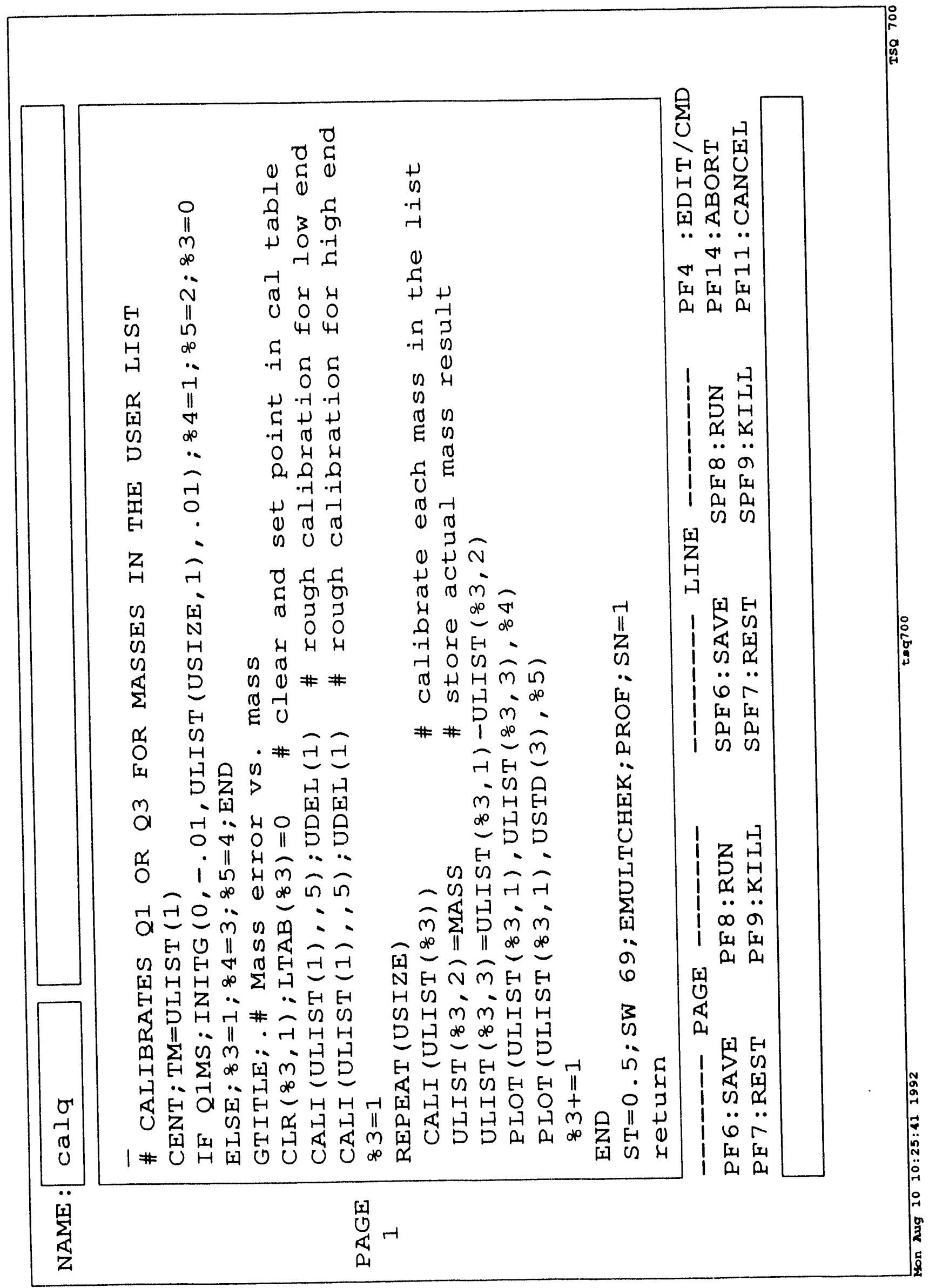




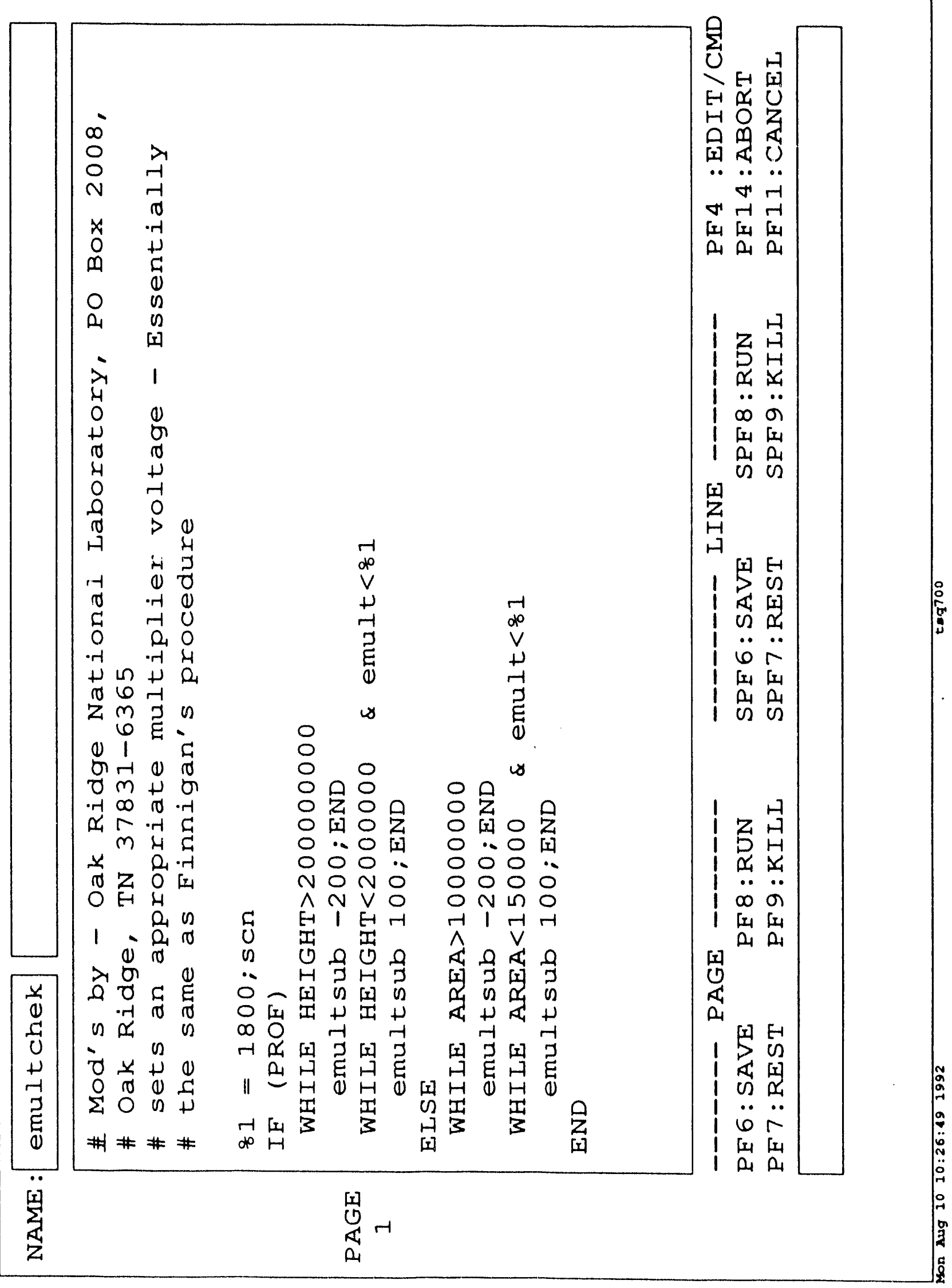




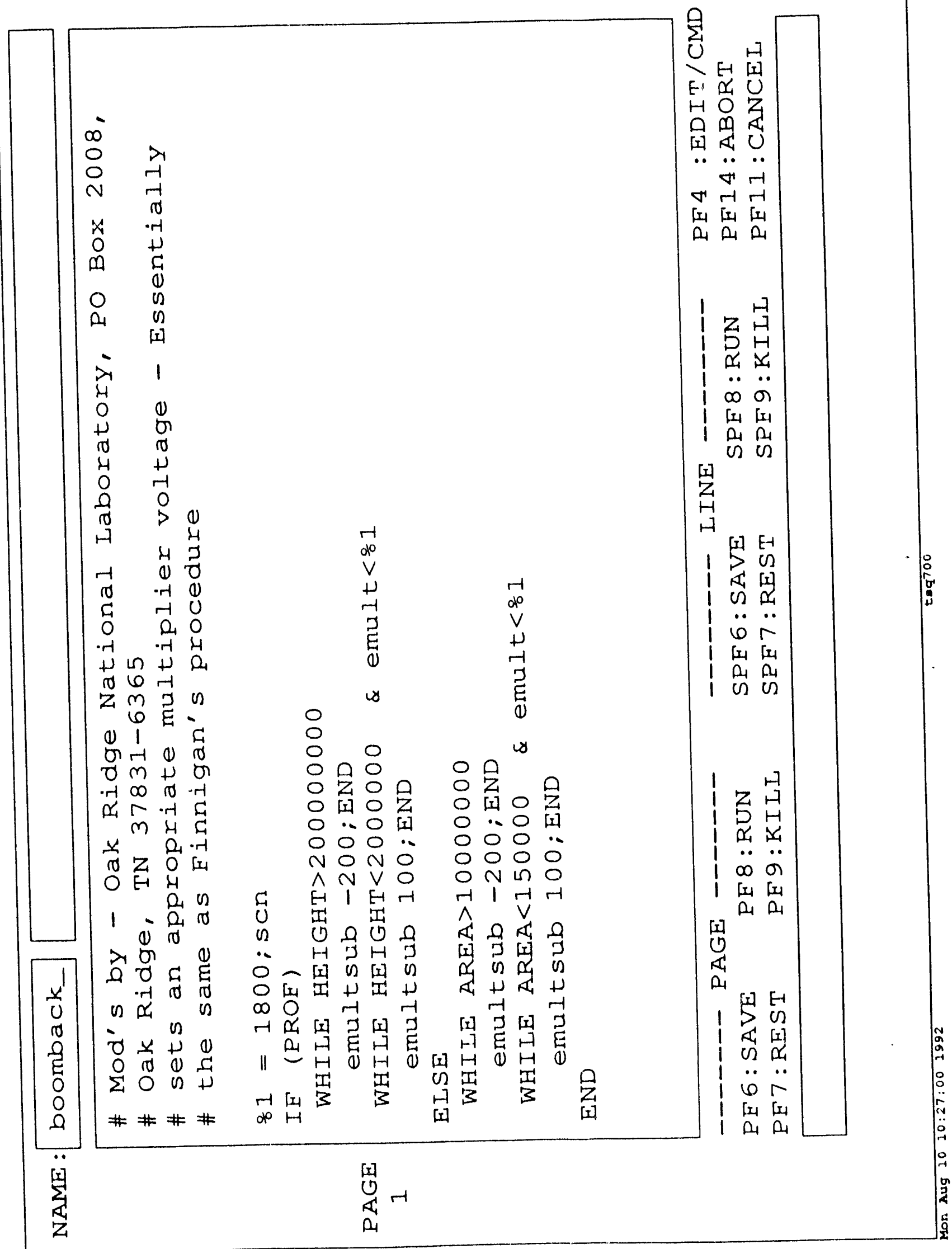




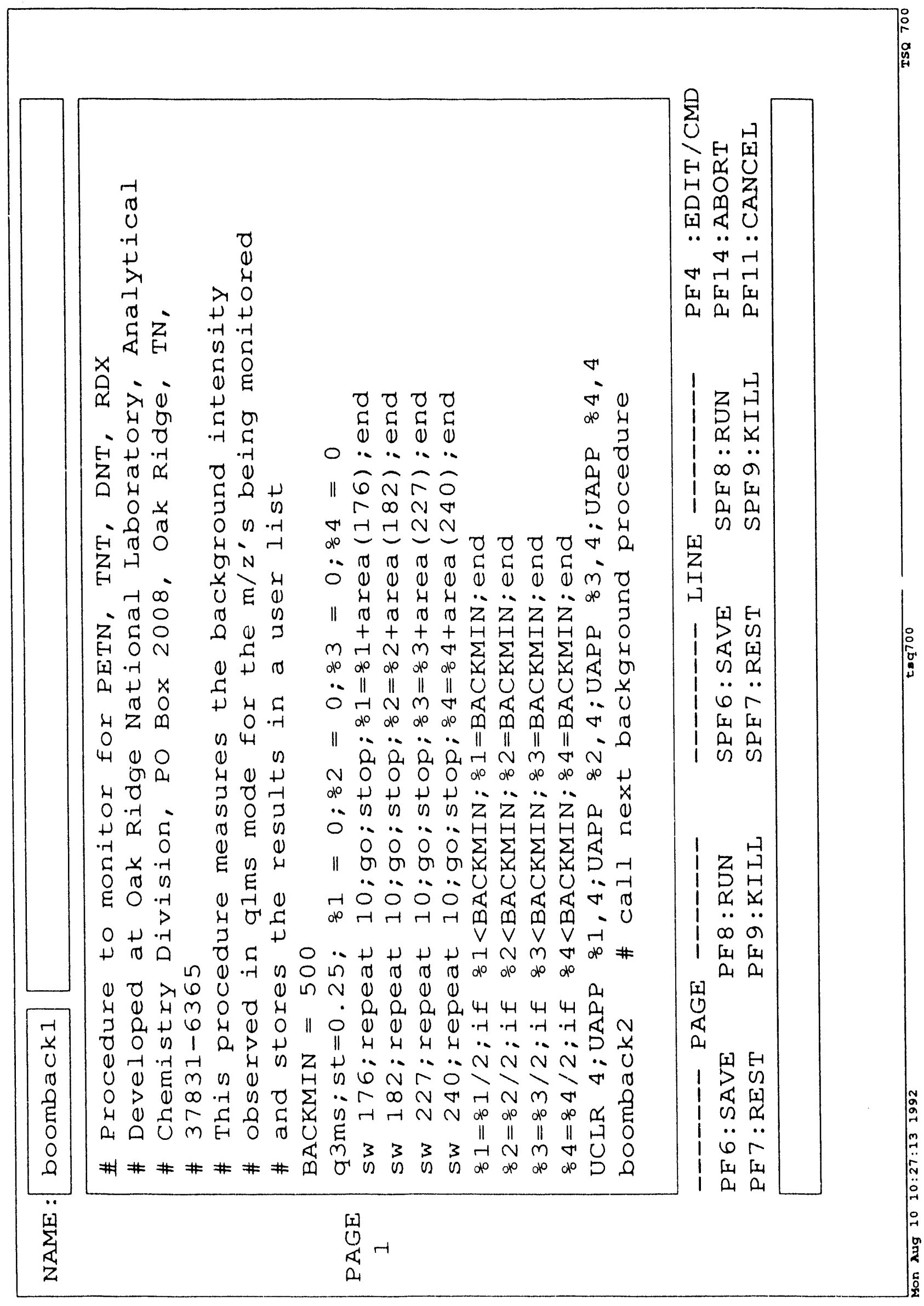




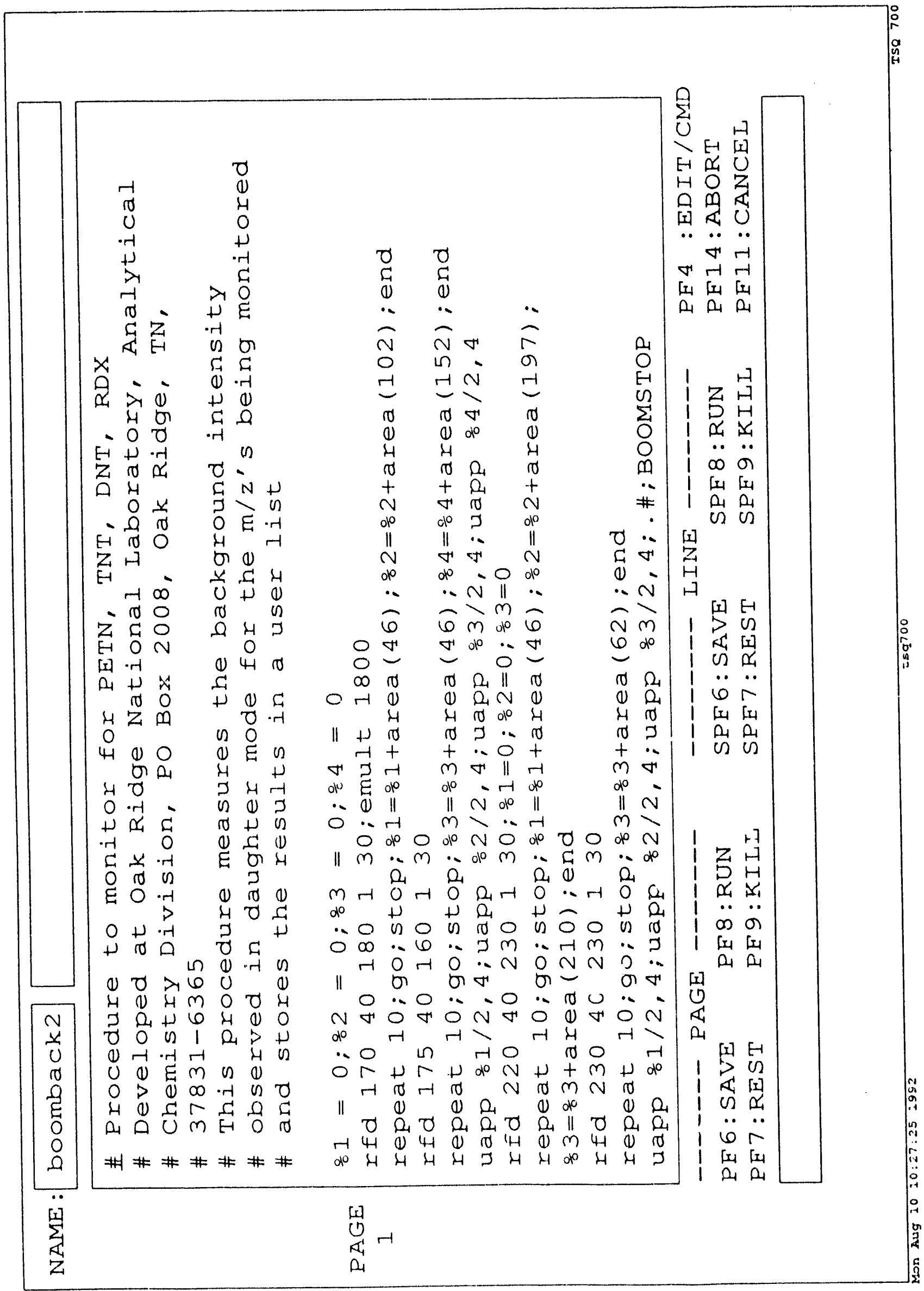




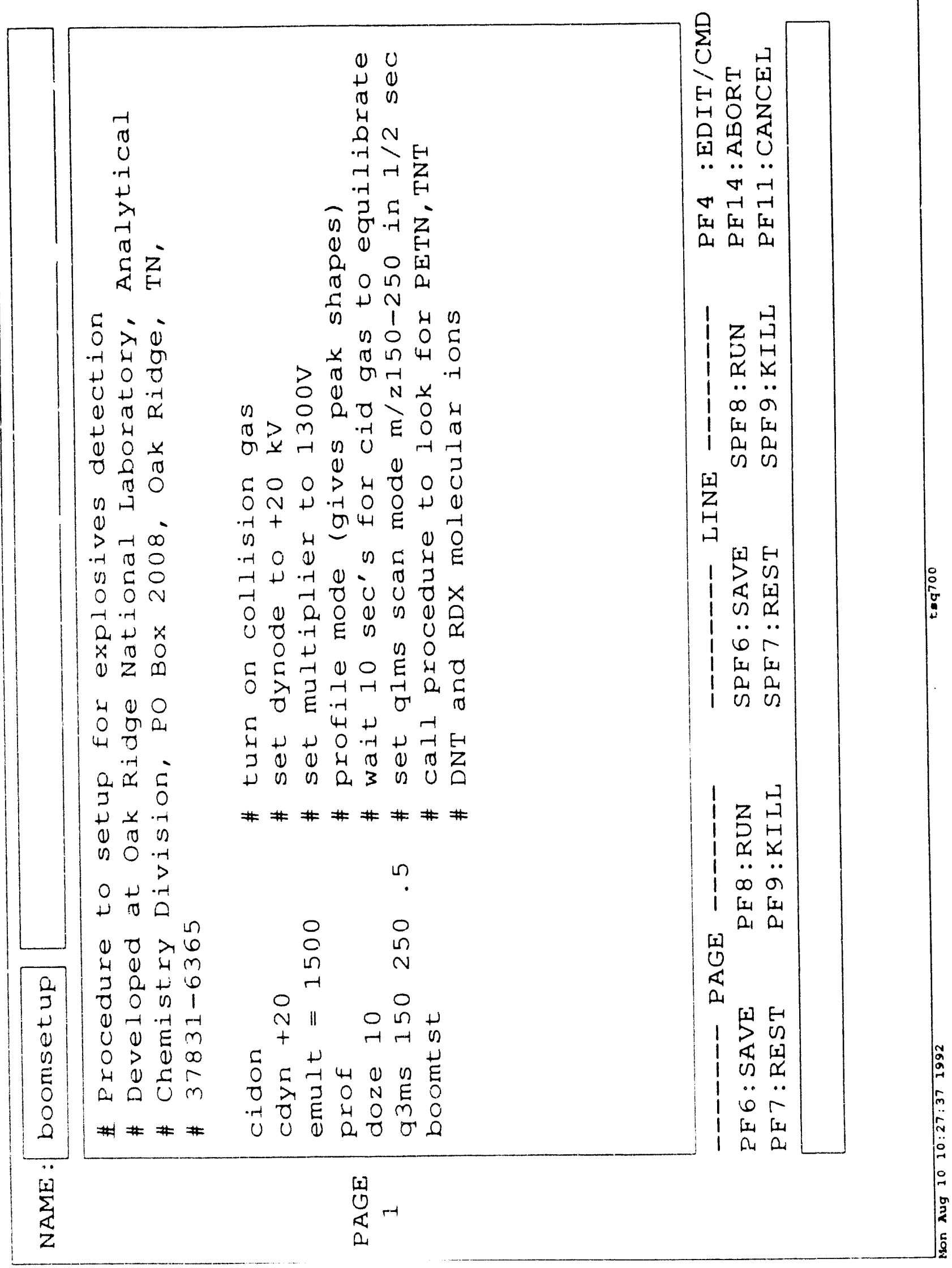




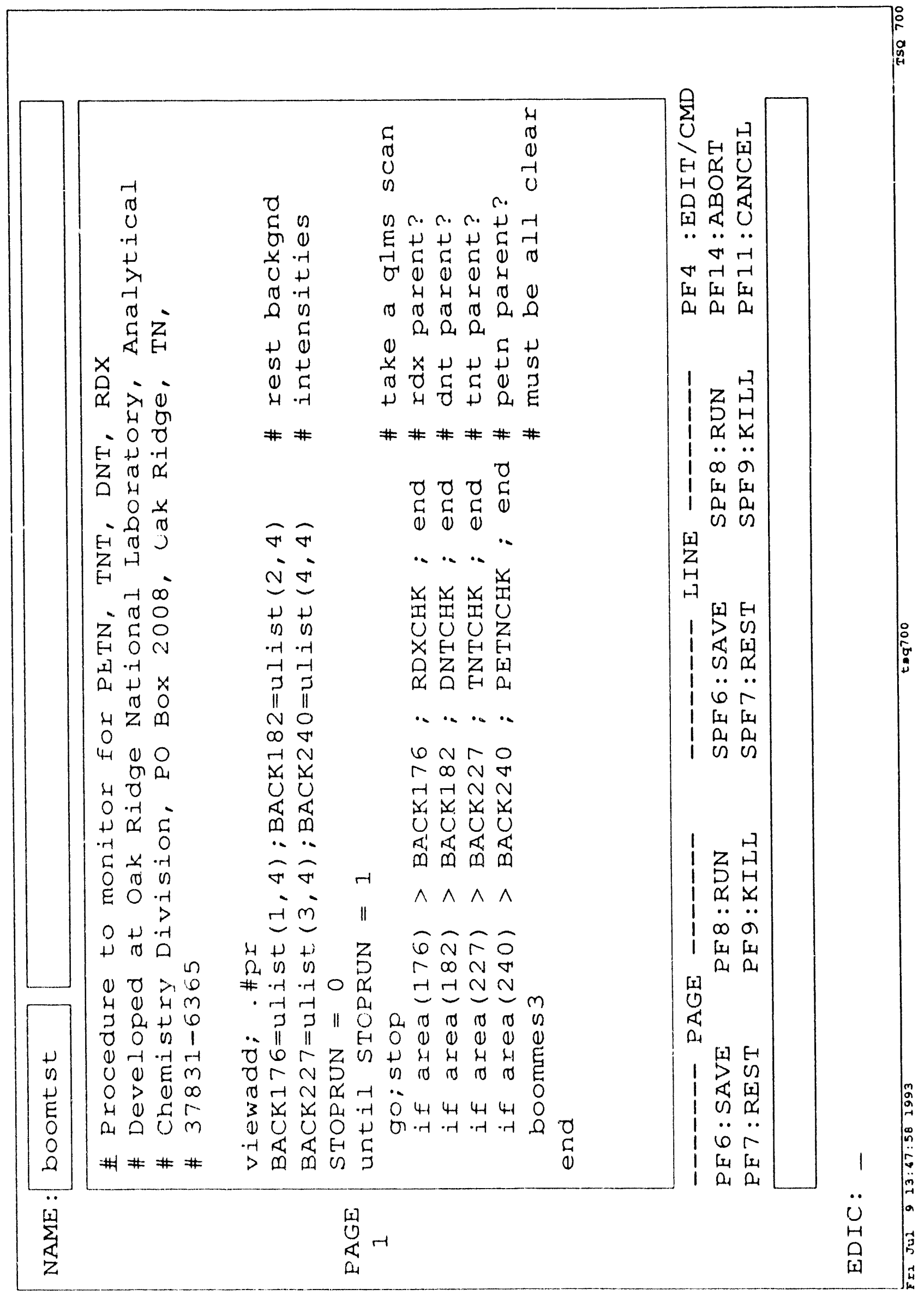




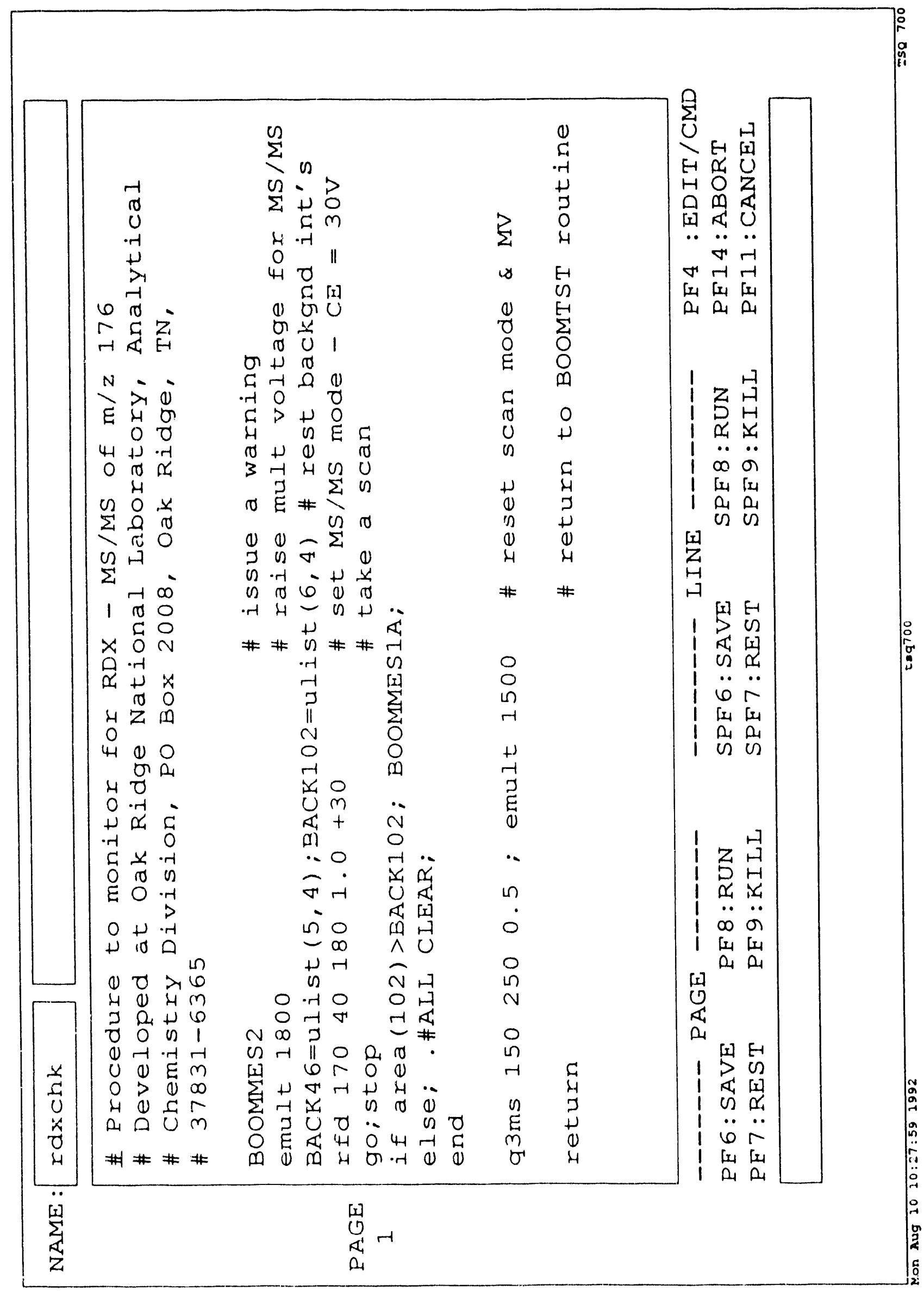




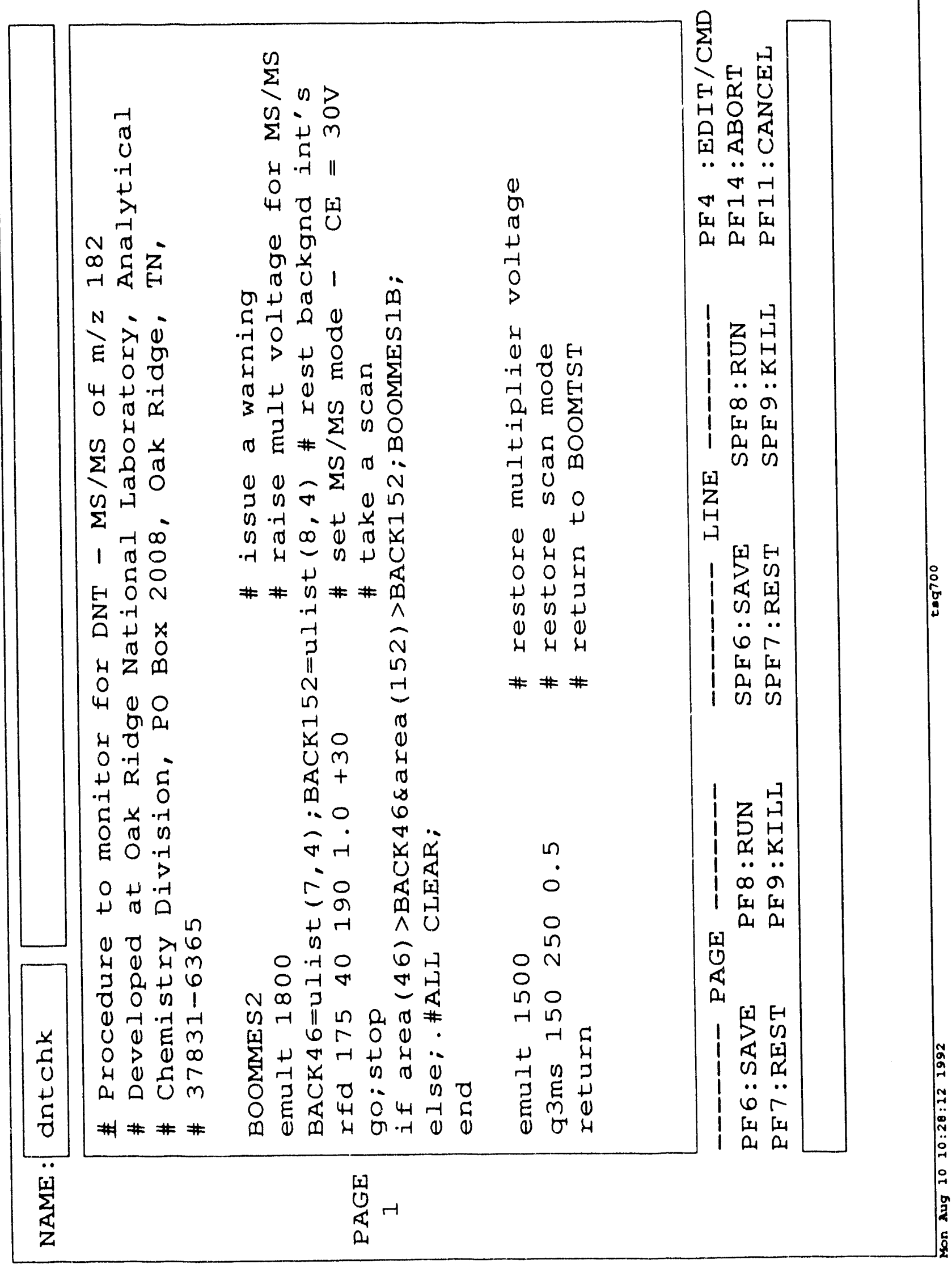




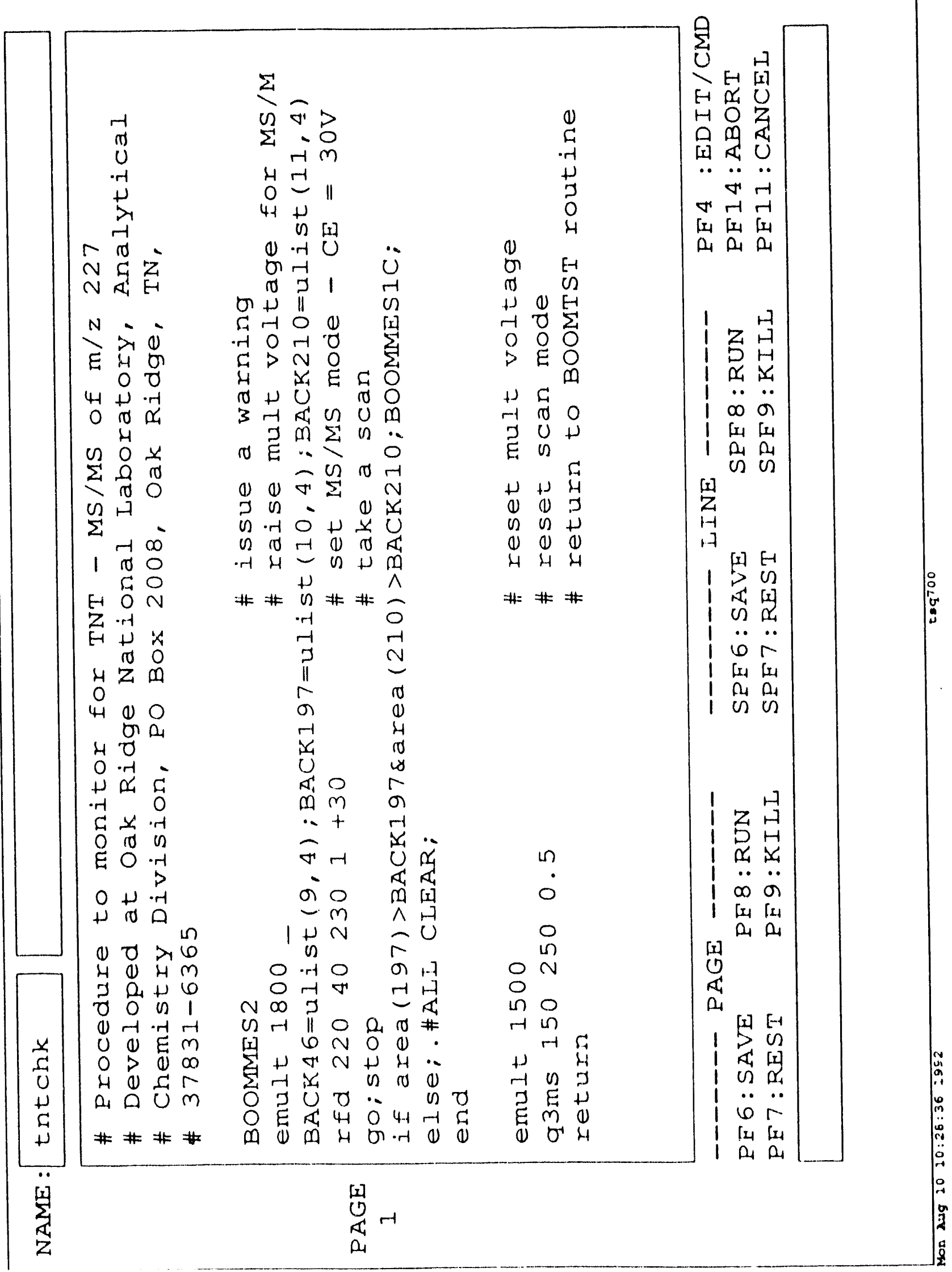




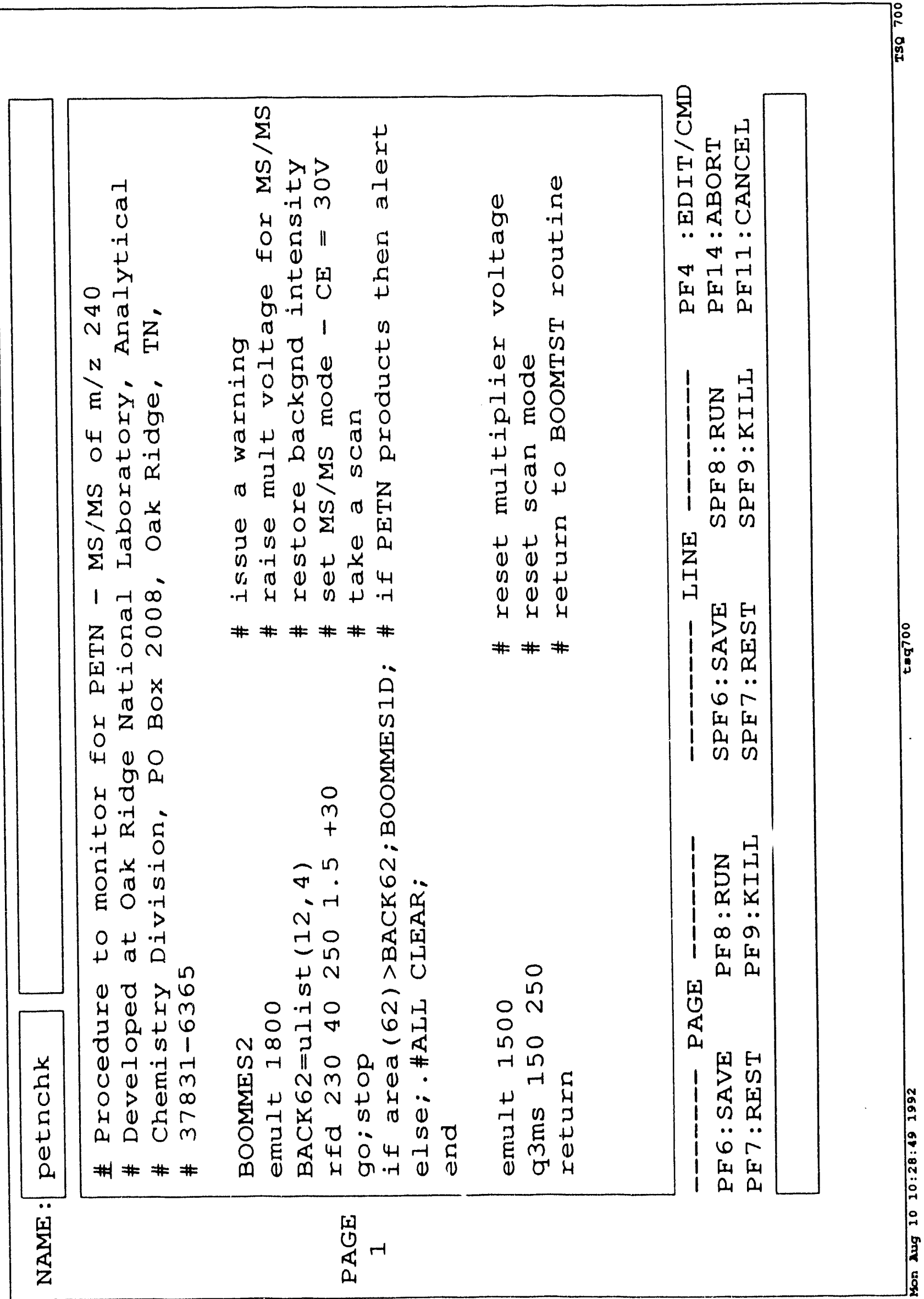




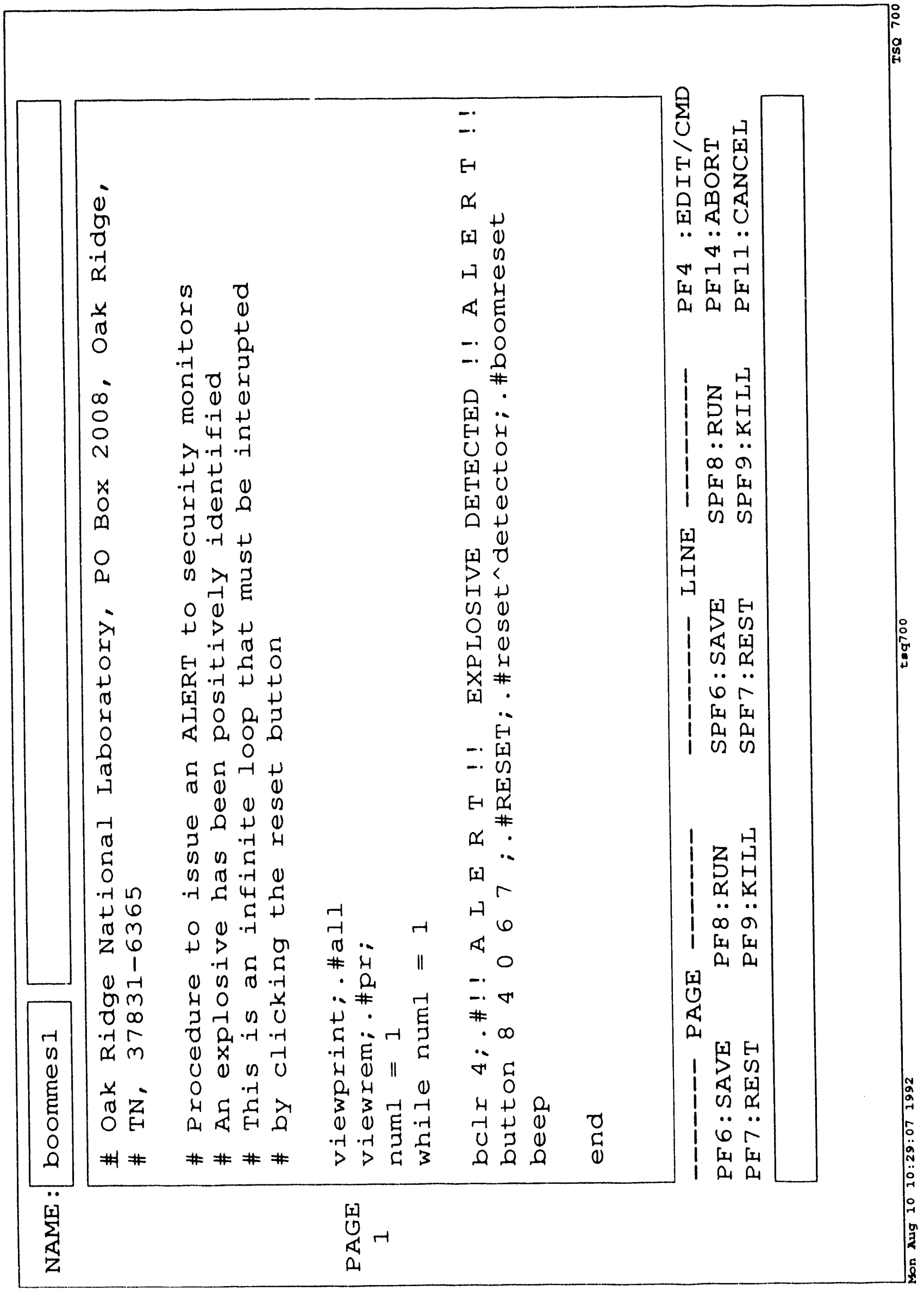




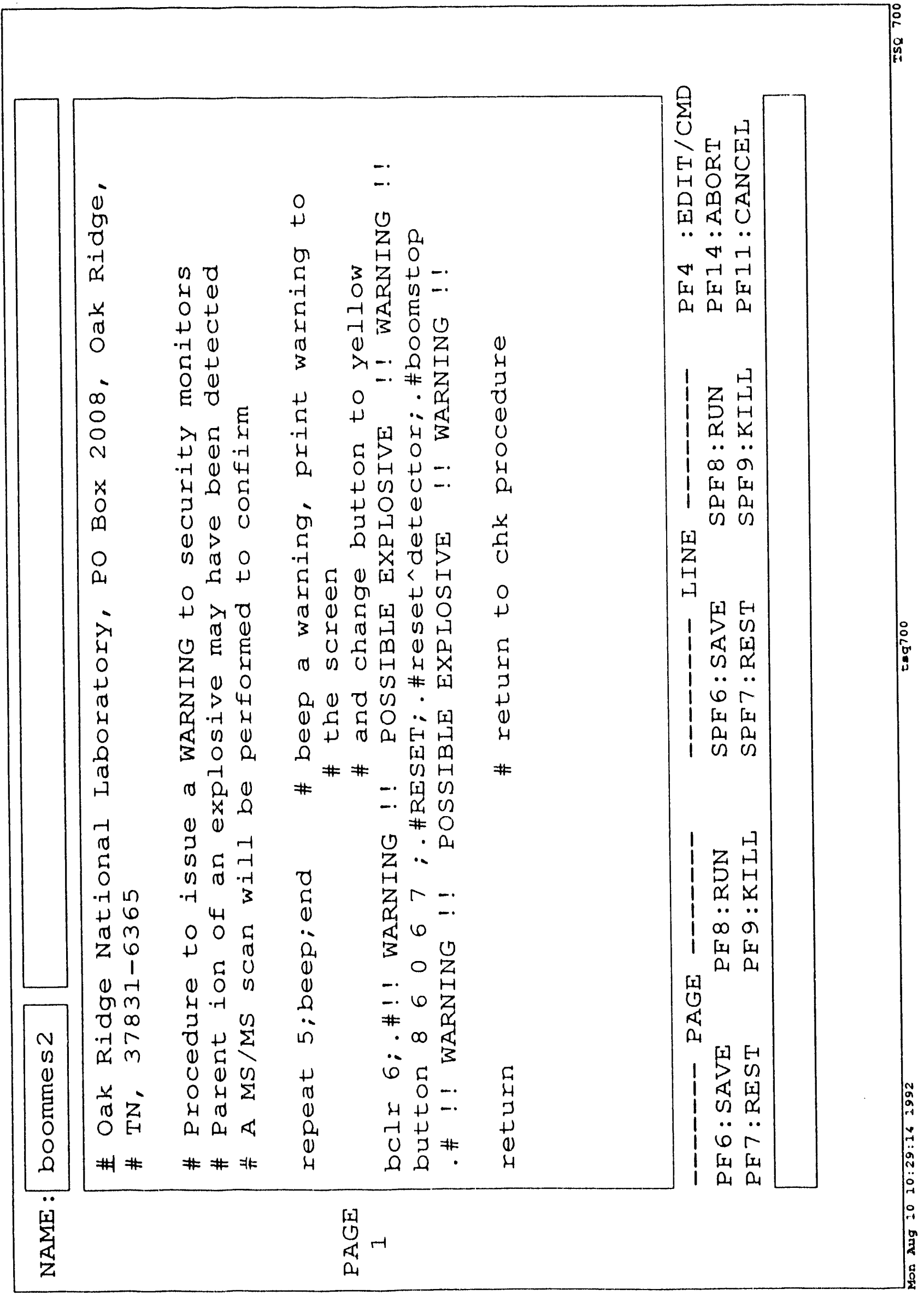




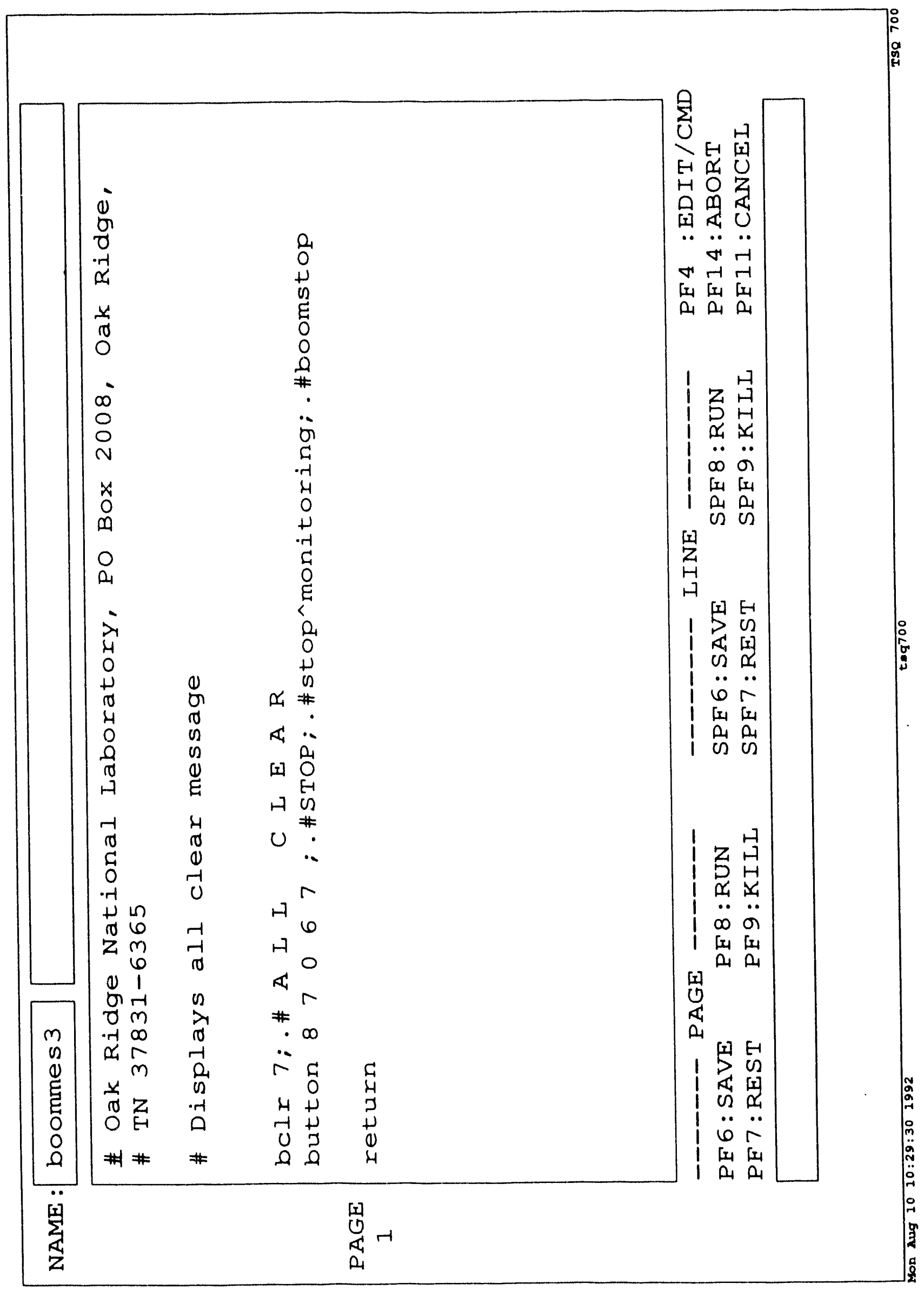




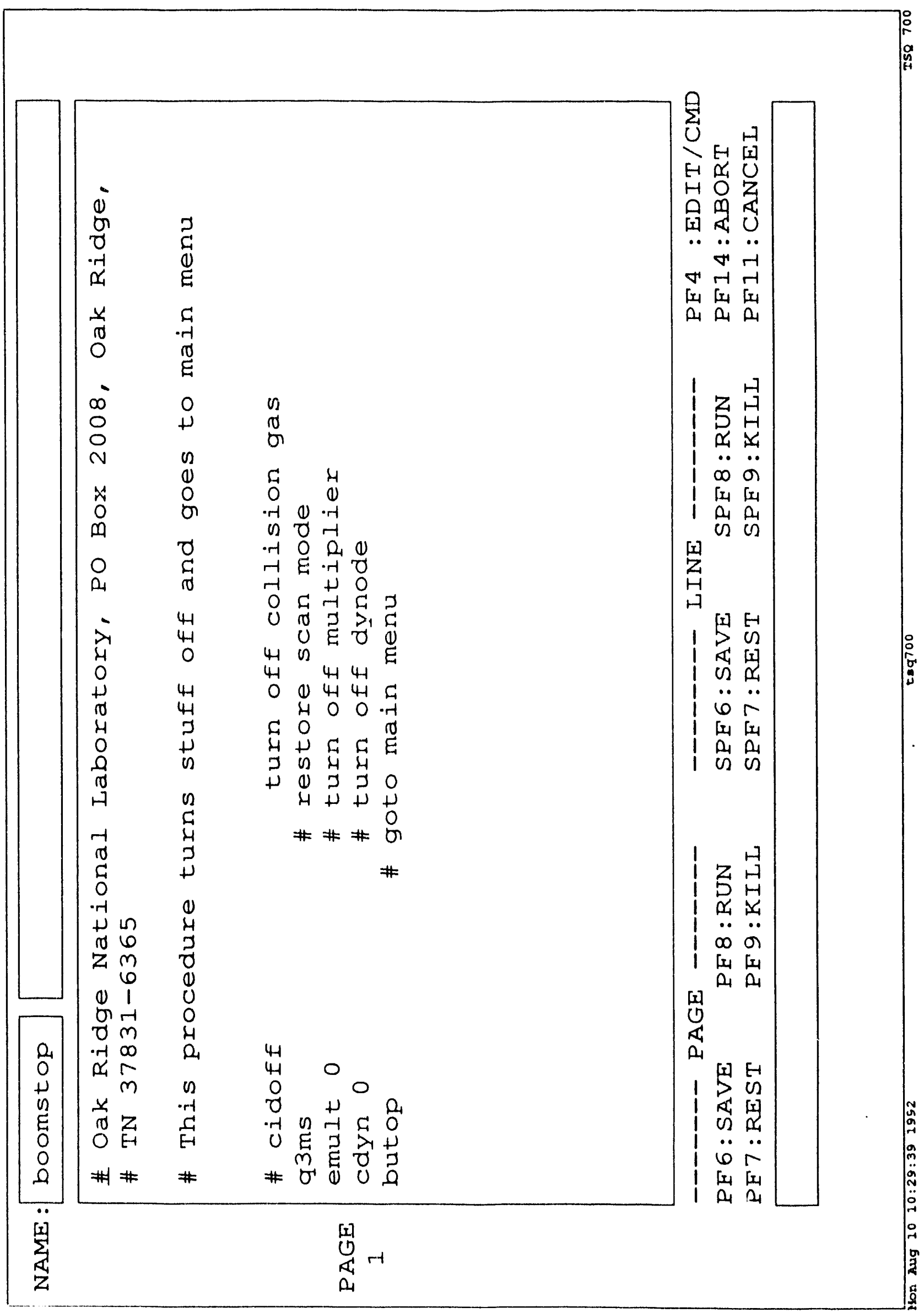




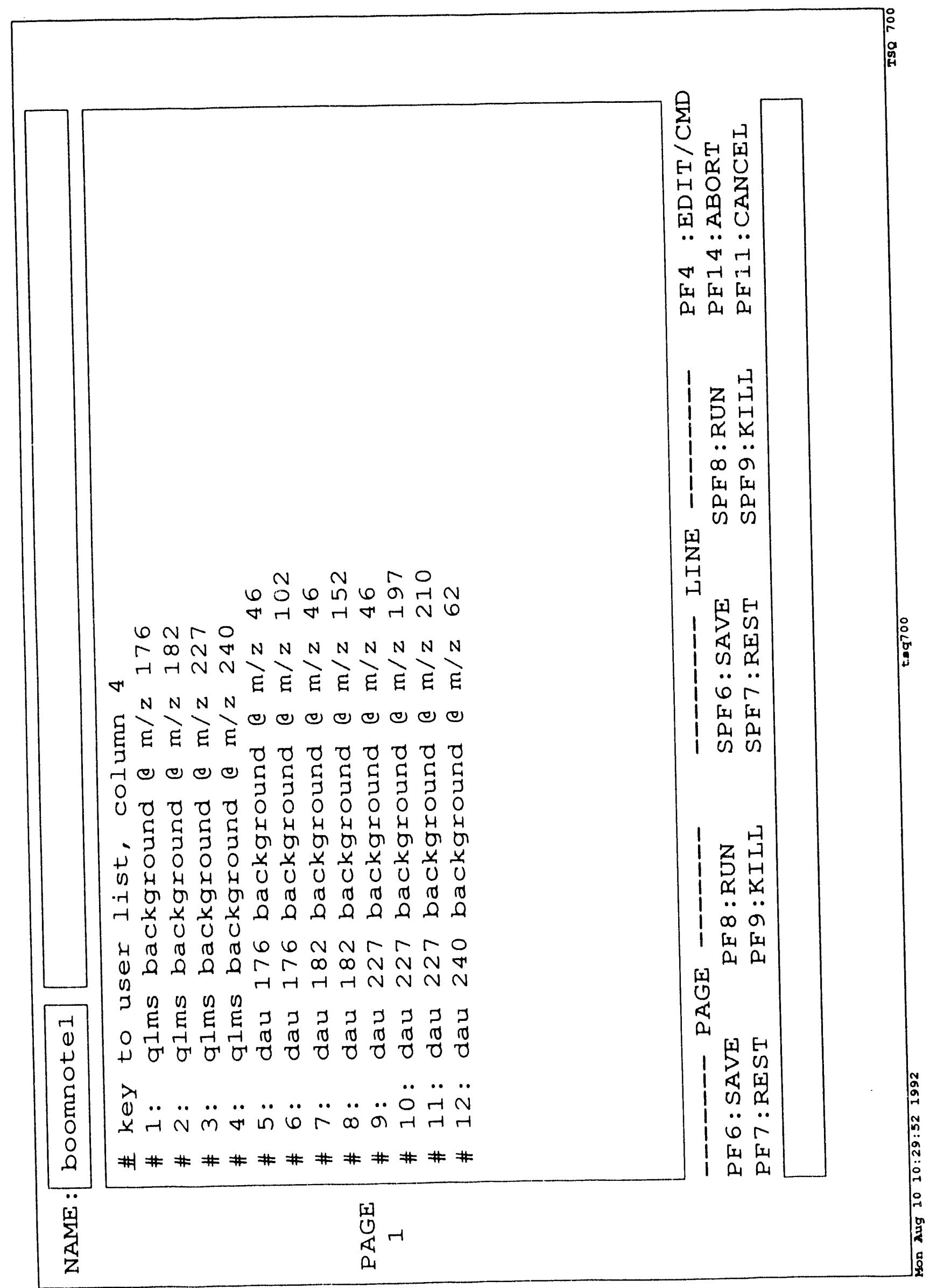




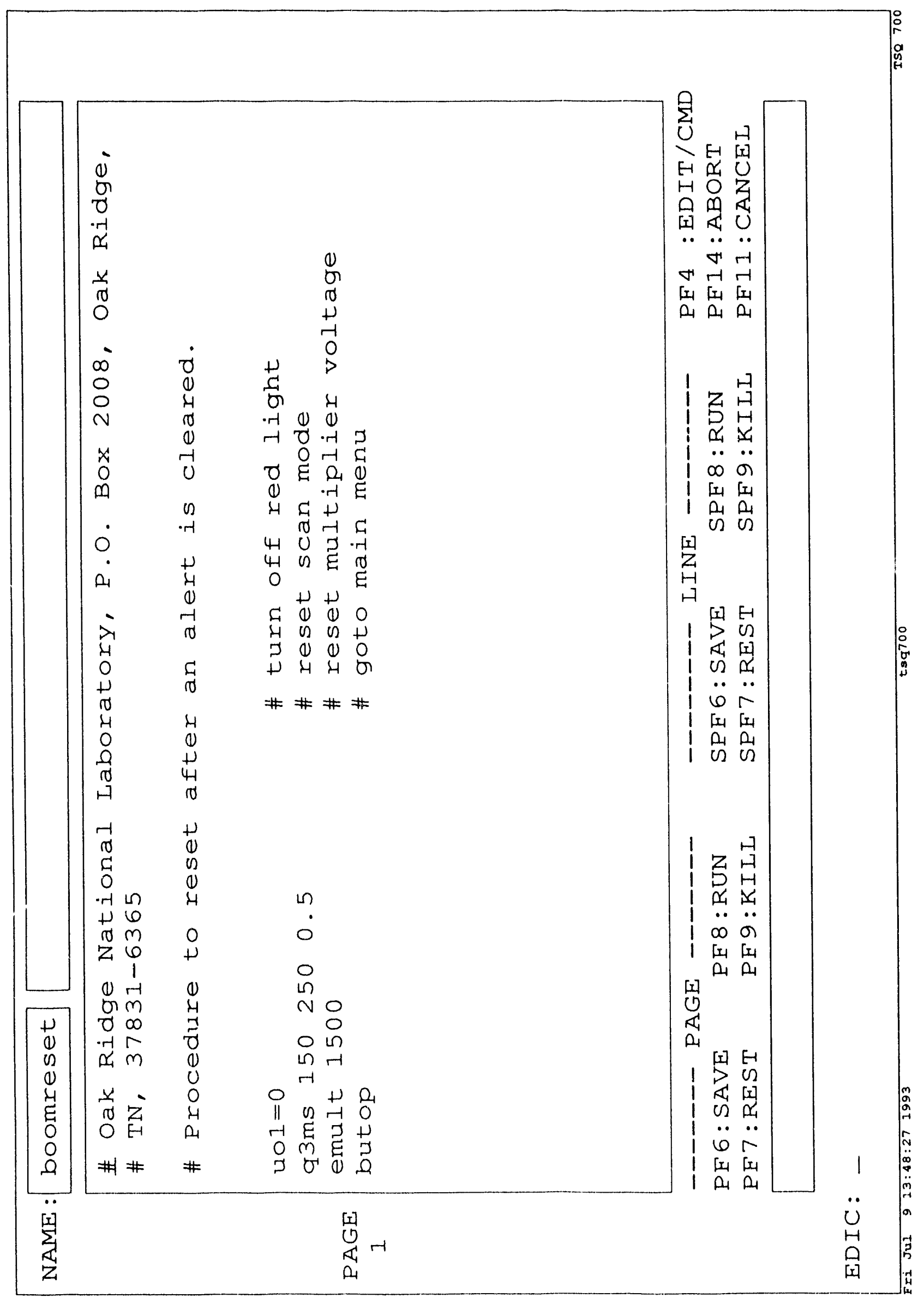




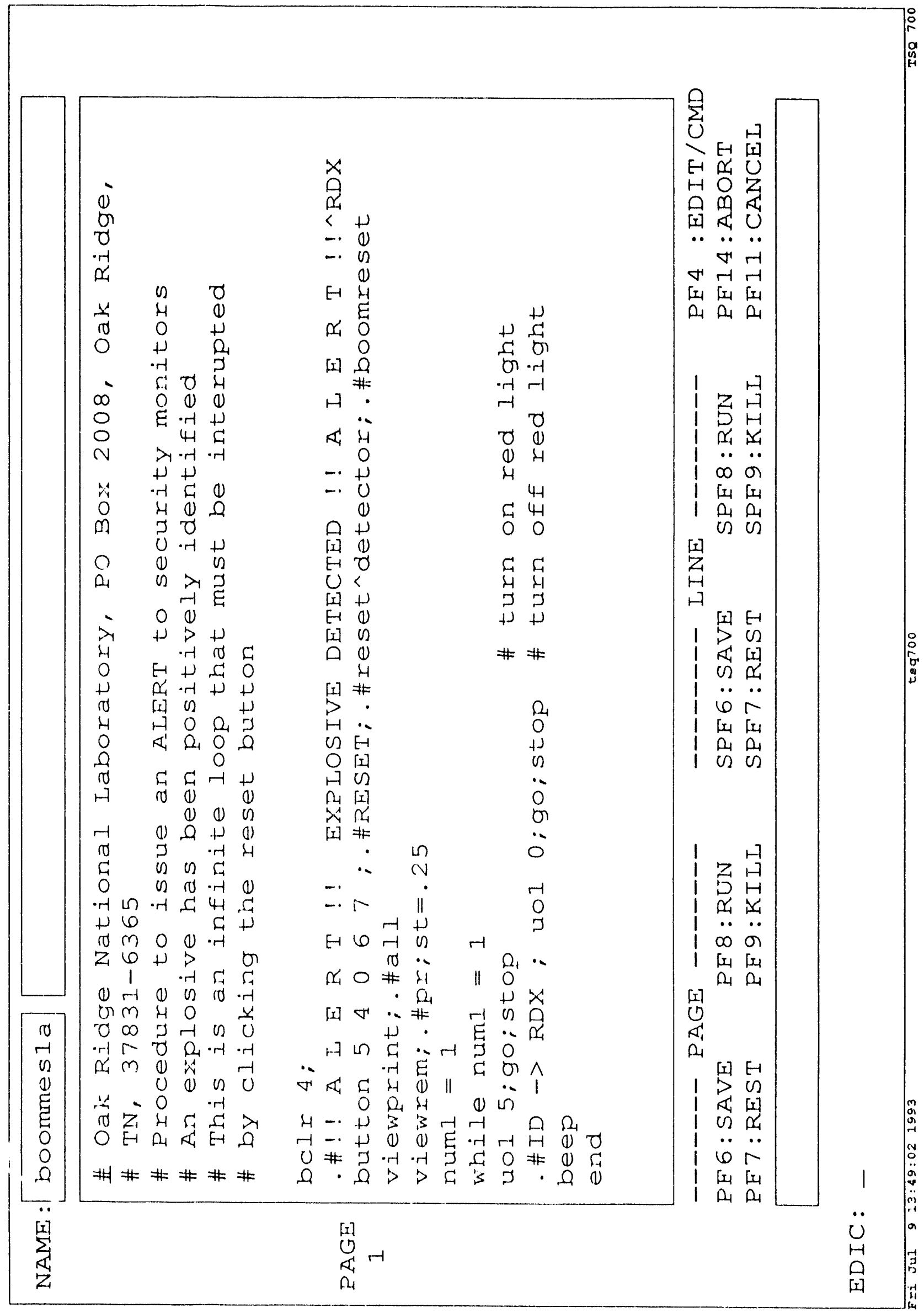




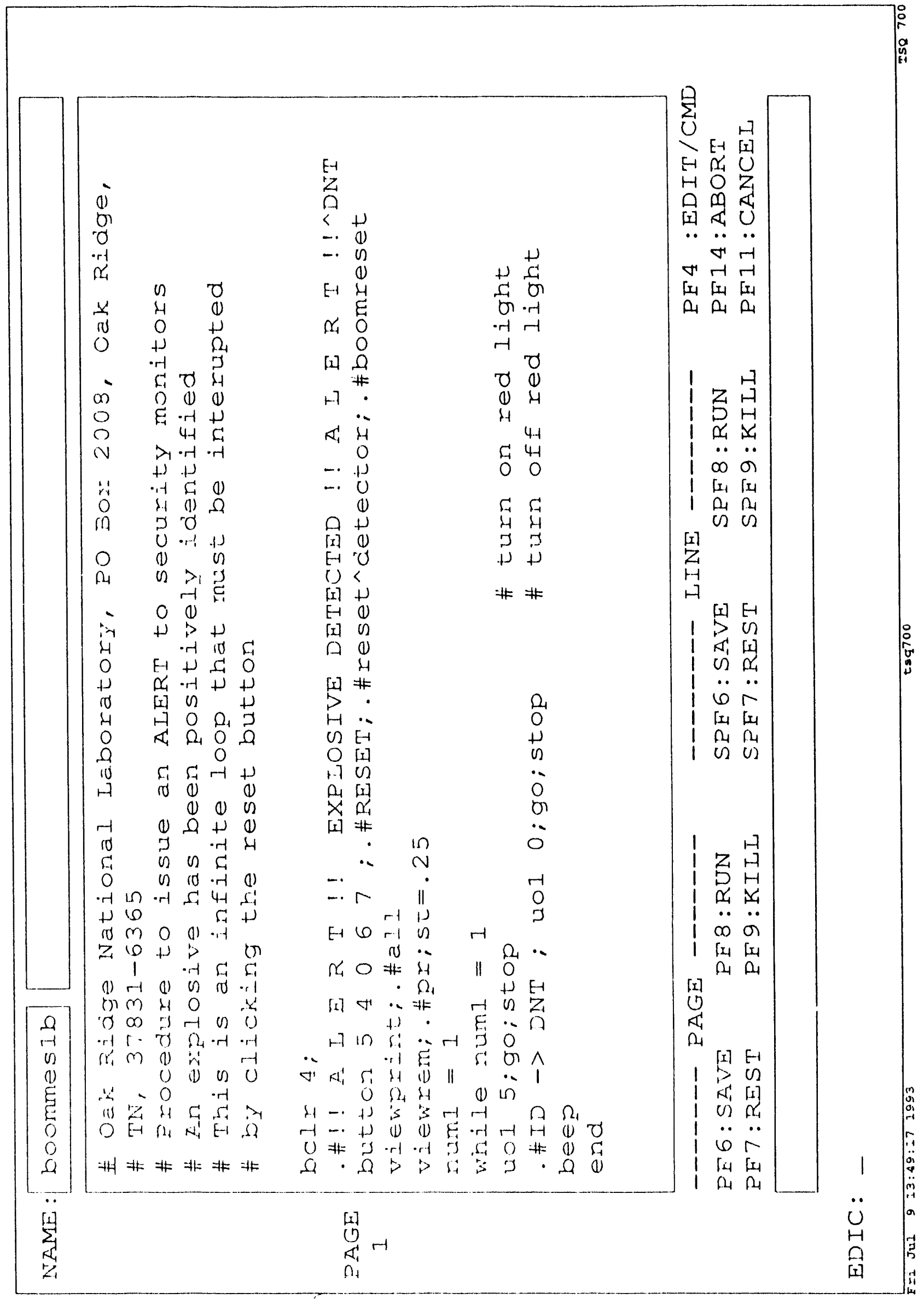




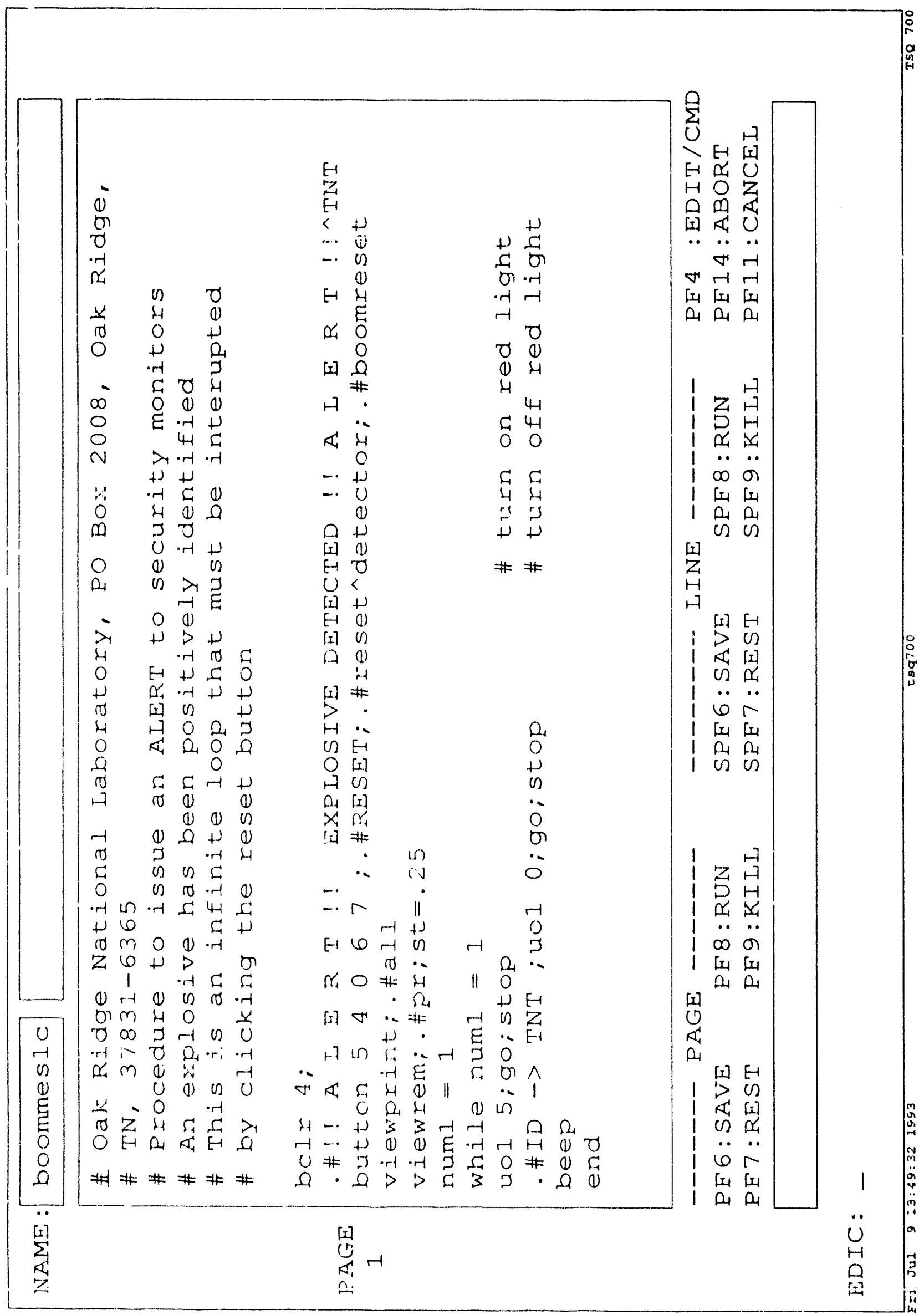




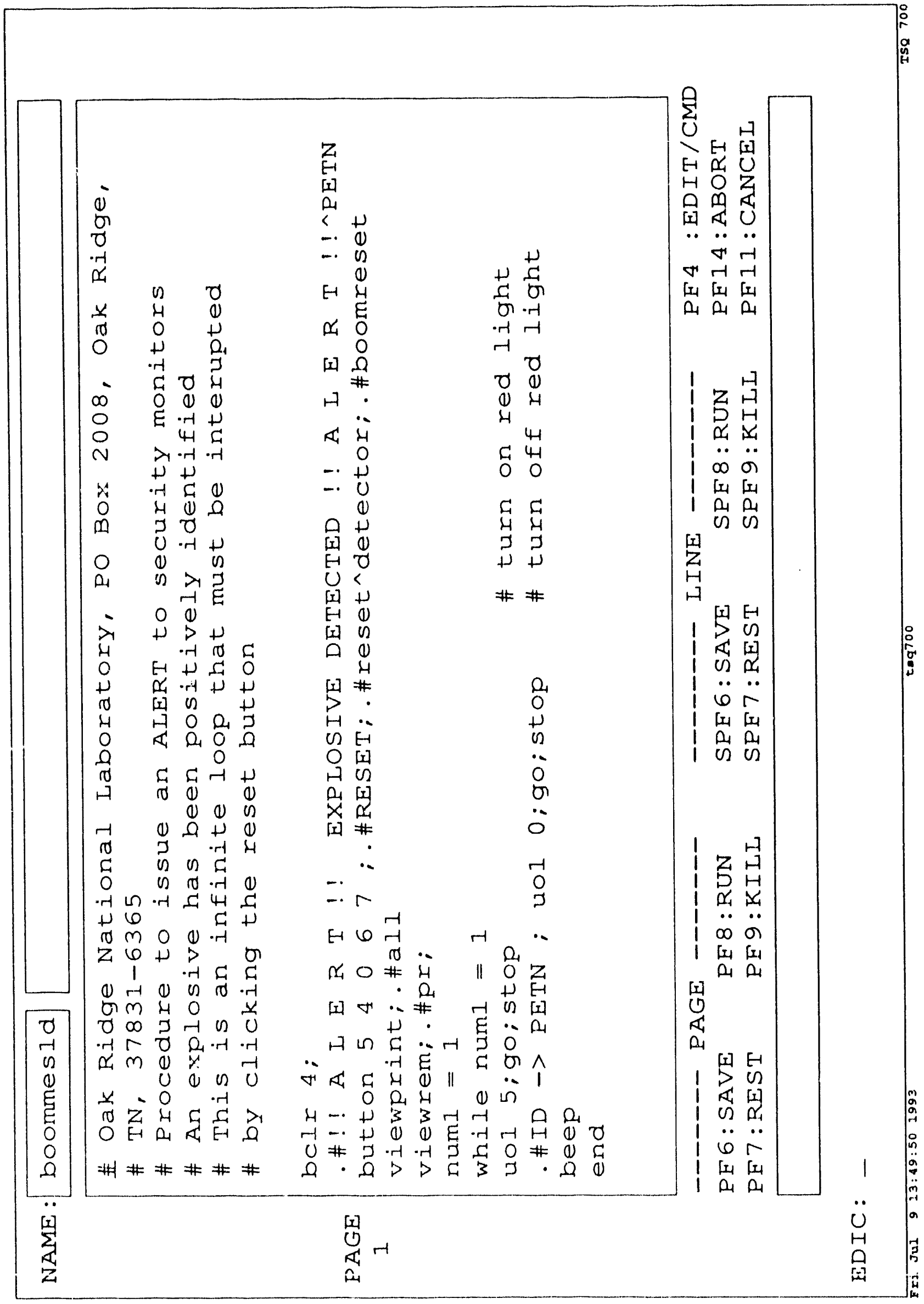




\section{INTERNAL DISTRIBUTION}

1. B. R. Appleton

2-6. K. G. Asano

7. M. V. Buchanan

8. J. E. Caton

$9 . \quad$ J. B. Dooley

10-14. D. E. Goeringer

15. W. H. Griest

16. M. R. Guerin

17. K. J. Hart

18. W. C. Kuykendall

19. T. A. MacDonnell

20. S. A. Mckenney

21-25. S. A. McLuckey

26. J. M. Ramsey

27-31. R. S. Ramsey

32. S. A. Sherrow

33. W. D. Shults

34-38. G. J. Van Berkel

39. M. B. Wise

40. Central Research Library

41. Document Reference Section

42-43. Laboratory Records

44. Laboratory Records - RC

45. Patent Section

\section{EXTERNAL DISTRIBUTION}

46. A. A. Cantu, U.S. Secret Service, Technical Security Division, 1800 G. Street, N.W., Washington, D.C. 20223

47. T. H. Chen, U.S. Army Armament Research, Development, and Engineering Center, Picatinny Arsenal, NJ 07806-5000

48. F. J. Conrad, Sandia National Laboratory, Albuquerque, NM 87185

49. J. P. Davies, Idaho National Engineering Laboratory, P.O. Box 1625, Idaho Falls, ID 83415

50. D. D. Fetterolf, Forensic Science Research Unit, FBI Academy, Quaritico, VA 22135

51. D. D. Garner, U.S. Bureau of Alcohol, Tobacco, and Firearms, Forensic Science Laboratory, 1401 Research Blvd., Rockville, MD 20850 
52-56. S. Hallowell, FAA Technical Center, Atlantic City Intl. Airport, Atlantic City, NJ 08405

57. J. R. Hobbs, Volpe National Transportation Systems Center, Research and Special Programs Administration, U.S. Dept. of Transportation, Kendall Square, Cambridge, MA 02142

58. D. Hoglund, U.S. Customs Service, Research and Development Office, 1301 Constitution Ave., N.W., Washington, D.C. 20229

59-63. G. Huston, FAA Technical Center, Atlantic City Intl. Airport, Atlantic City, NJ 08405

64-68. P. Jankowski, FAA Technical Center, Atlantic City Intl. Airport, Atlantic City, NJ 08405

69. W. E. Johnson, U.S. Customs Service, Research and Development Office, 1301 Constitution Ave., N.W., Washington, D.C. 20229

70. D. P. Lucero, Lucero and Associates, 18421 Cedar Drive, Triangle, VA 22172

71. W. J. McGann, Ion Track Instruments, Inc., 340 Fordham Road, Wilmington, MA 01887

72-76. A. Mercado, FAA Technical Center, Atlantic City Intl. Airport, Atlantic City, NJ 08405

77. C. R. Midkiff, Jr., U.S. Bureau of Alcohol, Tobacco, and Firearms, Forensic Science Laboratory, 1401 Research Blvd., Rockville, MD 20850

78. R. Nine, U.S. Dept. of Energy, Special Technologies Program, Office of Threat Assessment, Rm. GM-097, FORSTL, Washington, D.C. 20585

79. F. Whitehurst, Federal Bureau of Investigation, 9th and Penna. Ave., N.W., Washington, D.C. 20535

80. Yehuda Yinon, Dept. of Environmental Sciences and Energy Research, Weizmann Institute of Science, Rehovot, Israel

81-82. Office of Scientific and Technical Information, Oak Ridge, TN 37830

83. Assistant Manager of Energy Research and Development, Oak Ridge, TN 37830 
1
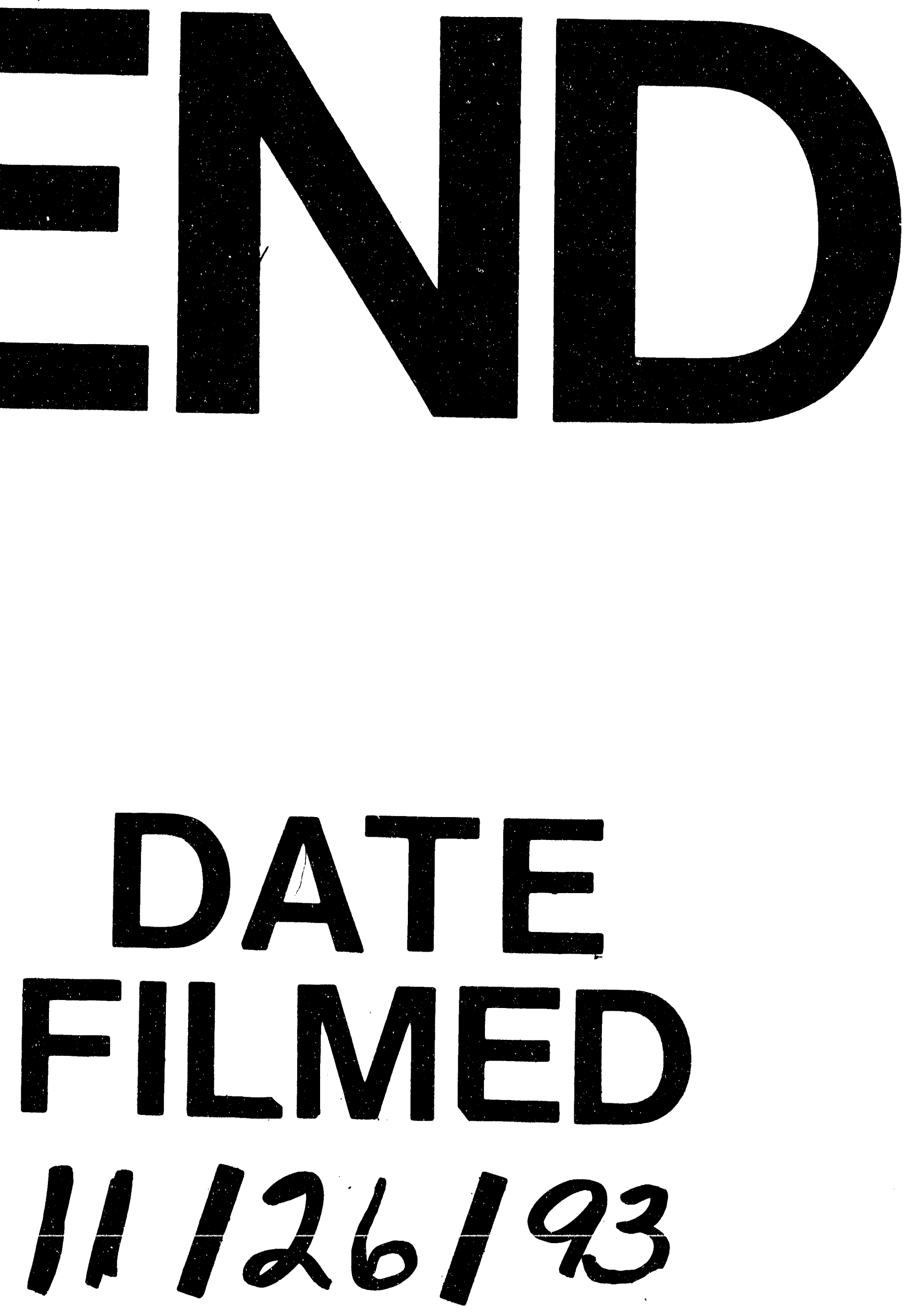
